\title{
Analysis of Reliability Experiments with Random Blocks and Subsampling
}

\author{
Jennifer L. K. Kensler \\ Dissertation submitted to the Faculty of the \\ Virginia Polytechnic Institute and State University \\ in partial fulfillment of the requirements for the degree of \\ Doctor of Philosophy \\ in \\ Statistics \\ G. Geoffrey Vining, Chair \\ Laura J. Freeman \\ Yili Hong \\ William H. Woodall
}

July 20, 2012

Blacksburg, Virginia

Keywords: Design of Experiments, Regression with Lifetime Data, Random Effects, Weibull Distribution

Copyright 2012, Jennifer L. K. Kensler 


\title{
Analysis of Reliability Experiments with Random Blocks and Subsampling
}

\author{
Jennifer L. K. Kensler
}

(ABSTRACT)

Reliability experiments provide important information regarding the life of a product, including how various factors may affect product life. Current analyses of reliability data usually assume a completely randomized design. However, reliability experiments frequently contain subsampling which is a restriction on randomization. A typical experiment involves applying treatments to test stands, with several items placed on each test stand. In addition, raw materials used in experiments are often produced in batches. In some cases one batch may not be large enough to provide materials for the entire experiment and more than one batch must be used. These batches lead to a design involving blocks. This dissertation proposes two methods for analyzing reliability experiments with random blocks and subsampling. The first method is a two-stage method which can be implemented in software used by most practitioners, but has some limitations. Therefore, a more rigorous nonlinear mixed model method is proposed. 


\section{Contents}

1 Introduction $\quad 1$

1.1 The Weibull Distribution . . . . . . . . . . . . . . . . . 2

1.2 Censoring . . . . . . . . . . . . . . . . . . . 5

1.3 Experimental Design . . . . . . . . . . . . . . . . . 6

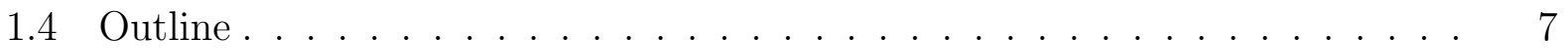

2 Literature Review $\quad 9$

2.1 Analysis of Lifetime Data . . . . . . . . . . . . . . . . . . . . 9

2.1.1 Location-Scale and Log-Location-Scale Distributions . . . . . . . . . 10

2.1.2 The Weibull and Smallest Extreme Value Distributions . . . . . . . . 11

2.1.3 Maximum Likelihood Estimation . . . . . . . . . . . . . 12 
2.1.4 Bias of Maximum Likelihood Estimates of the Weibull Shape Parameter for Small Sample Sizes _. . . . . . . . . . . . . . . . 14

2.1.5 Inference Based on the Likelihood . . . . . . . . . . . . 17

2.1.6 Inference Based on Normal Theory . . . . . . . . . . . . . . . . 19

2.1.7 Regression with Lifetime Data . . . . . . . . . . . . . . . 22

2.2 Design of Experiments . . . . . . . . . . . . . . . 25

$2.2 .1 \quad$ Subsampling . . . . . . . . . . . . . . . . . . . 25

2.2 .2 Random Blocks . . . . . . . . . . . . . . . . . . . . . . 26

2.3 Reliability Experiments with Subsampling . . . . . . . . . . . 28

2.3 .1 Two-Stage Method for Subsampling . . . . . . . . . . . . . . 28

3 Reliability Experiments with Subsampling 35

3.1 Simulation Study . . . . . . . . . . . . . . . . . . . . 36

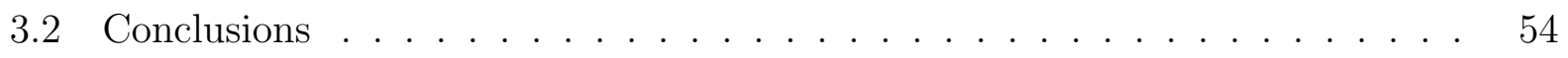

4 Two-Stage Analysis $\quad 56$

4.1 Random Blocks with Subsampling . . . . . . . . . . . . . . . 56

4.2 Two-Stage Method: Random Blocks with Subsampling . . . . . . . . . . 58

4.3 An Illustrative Example . . . . . . . . . . . . . . . . . . . 61 
4.4 Simulation Study . . . . . . . . . . . . . . . . . . . . . . 63

4.5 Conclusions for Two-Stage Random Block Analysis . . . . . . . . . . . 75

$\begin{array}{lll}5 & \text { Joint-Likelihood Analysis } & 76\end{array}$

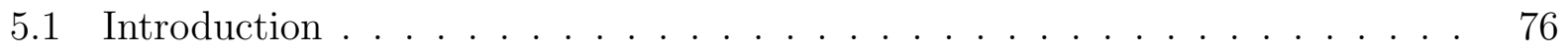

5.2 The Model . . . . . . . . . . . . . . . . . . . . . . . . . 77

5.3 Gauss-Hermite Quadrature . . . . . . . . . . . . . . . . . . . . . . 79

5.4 Inference . . . . . . . . . . . . . . . . . . . . 84

5.4 .1 Wald Inferences . . . . . . . . . . . . . . . . . 84

5.4 .2 Inference Based on the Likelihood . . . . . . . . . . . . . . 87

5.5 An Illustrative Example $\ldots \ldots$

5.6 Software for the NLMM Solution . . . . . . . . . . . . . . . . 92

$\begin{array}{llr}6 & \text { Simulation Studies } & 94\end{array}$

6.1 Parameter Estimation Study . . . . . . . . . . . . . . . . . . . . . 94

6.2 Simulation Study for Percentiles . . . . . . . . . . . . . . . 116

6.3 Conclusions from Simulation Studies _ . . . . . . . . . . . . . . 125

$\begin{array}{llr}7 & \text { Conclusions and Future Work } & 126\end{array}$ 
7.1 Conclusions . . . . . . . . . . . . . . . . . . . . . 126

7.2 Future Work . . . . . . . . . . . . . . . . . . . . . . . 127

Bibliography

A Two-Stage Simulation Study Tables and Figures

A.1 Additional Tables for the Two-Stage Simulation Study . . . . . . . . . . 134

A.2 Figures for the Two-Stage Simulation Study . . . . . . . . . . . 137

$\begin{array}{ll}\text { B Derivation of Variance-Covariance Matrix } & 144\end{array}$

$\begin{array}{lr}\text { C R and SAS Code } & 159\end{array}$

C.1 R Code . . . . . . . . . . . . . . . . . . . . . . . . . . . . . . . 159

C.2 SAS Code . . . . . . . . . . . . . . . . . . 167

$\begin{array}{lr}\text { D Weibull Variability } & 169\end{array}$ 


\section{List of Figures}

1.1 Probability Distribution Function of the Weibull Distribution $(\eta=100)$. . 3

1.2 Hazard Function of the Weibull Distribution $(\eta=100) \ldots \ldots$

3.1 Ratio of $\hat{\beta}$ to $\beta \ldots \ldots \ldots \ldots \ldots \ldots$

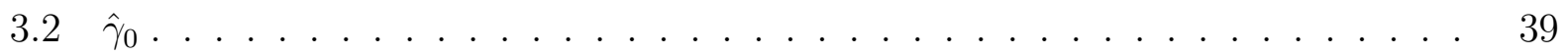

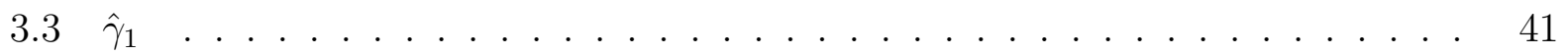

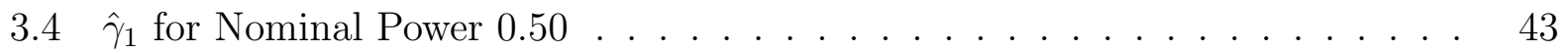

$3.5 \hat{\gamma}_{1}$ for Nominal Power $0.80 \ldots \ldots \ldots$. . . . . . . . . . . 44

$3.6 \hat{\gamma}_{1}$ for Nominal Power $0.90 \ldots \ldots . \ldots \ldots$. . . . . . . . . . 45

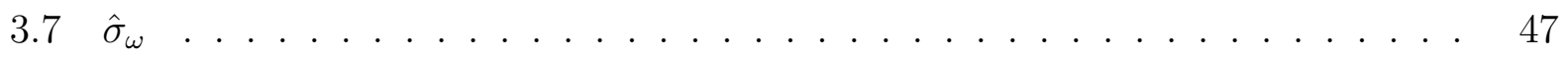

3.8 Empirical Error Rate . . . . . . . . . . . . . . . . . . . 49

3.9 Nominal Power of $0.50 \ldots \ldots \ldots \ldots 1$

3.10 Nominal Power of $0.80 \ldots \ldots$. . . . . . . . . . . . . . . 52 
3.11 Nominal Power of $0.90 \ldots \ldots \ldots \ldots$

4.1 Experimental Setup . . . . . . . . . . . . . . . . . . . . . . . 57

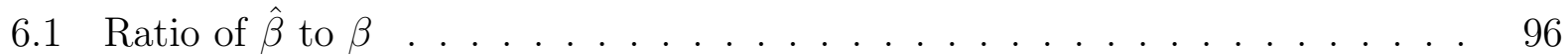

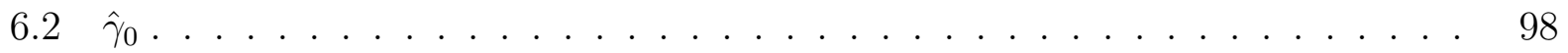

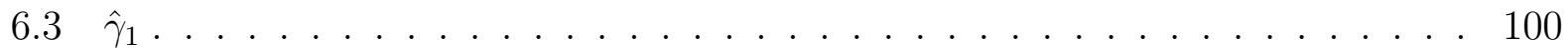

$6.4 \hat{\gamma}_{1}$ for Nominal Power $0.50 \ldots \ldots \ldots$

$6.5 \hat{\gamma}_{1}$ for Nominal Power $0.80 \ldots \ldots \ldots$

$6.6 \hat{\gamma}_{1}$ for Nominal Power $0.90 \ldots \ldots \ldots$

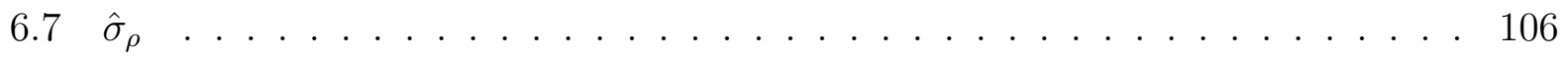

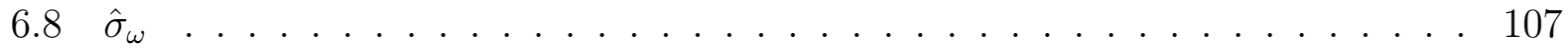

6.9 Empirical Error Rate . . . . . . . . . . . . . . . . . . . . . . . . 109

6.10 Nominal Power of $0.50 \ldots \ldots \ldots 111$

6.11 Nominal Power of $0.80 \ldots \ldots \ldots$

6.12 Nominal Power of $0.90 \ldots \ldots \ldots$

$6.13 \frac{\hat{t}_{0.1}(70)}{t_{0.1}(70)}$ Under the Null Model . . . . . . . . . . . . . . . . . . . 118

$6.14 \frac{\hat{t}_{0.1}(70)}{t_{0.1}(70)}$ Under the Alternative Model . . . . . . . . . . . . . . . 120

6.15 Empirical Error Rate for Testing $t_{0.1} \ldots \ldots \ldots \ldots$ 
6.16 Empirical Power for Testing $t_{0.1} \ldots \ldots \ldots \ldots \ldots$

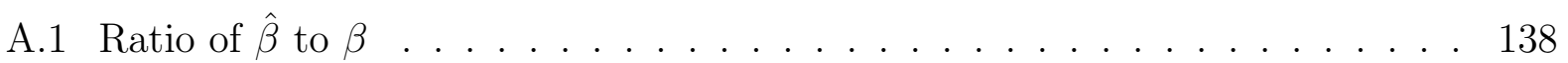

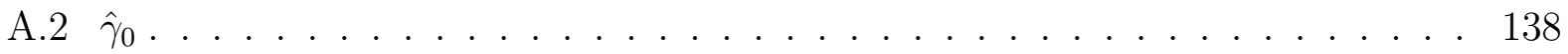

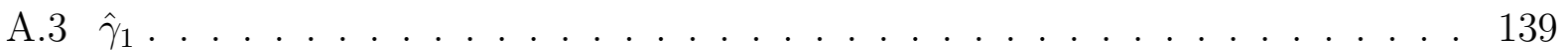

A.4 $\hat{\gamma}_{1}$ for Nominal Power of $0.50 \ldots \ldots \ldots \ldots \ldots$

A.5 $\hat{\gamma}_{1}$ for Nominal Power of $0.80 \ldots \ldots \ldots \ldots \ldots$

A.6 $\hat{\gamma}_{1}$ for Nominal Power of $0.90 \ldots \ldots \ldots \ldots$

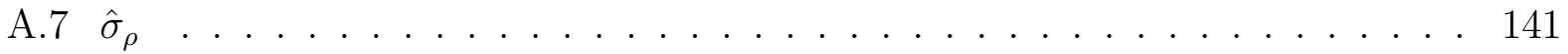

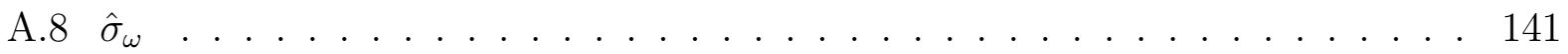

A.9 Empirical Error Rate . . . . . . . . . . . . . . . . . . . . . . 142

A.10 Nominal Power of $0.50 \ldots \ldots \ldots$

A.11 Nominal Power of $0.80 \ldots \ldots$. . . . . . . . . . . . . . . 143

A.12 Nominal Power of $0.90 \ldots \ldots \ldots$

D.1 Test Stand Standard Deviation by $\beta \ldots \ldots \ldots$

D.2 Test Stand Standard Deviation by $x \ldots \ldots \ldots \ldots \ldots$

D.3 Test Stand Standard Deviation by $x$ and $\beta \ldots \ldots \ldots \ldots$

D.4 Test Stand Standard Deviation by $\sigma_{\rho} \ldots \ldots \ldots \ldots \ldots \ldots$ 
D.5 Test Stand Standard Deviation by $\sigma_{\rho}$ and $\beta \ldots \ldots \ldots \ldots \ldots$

D.6 Test Stand Standard Deviation by $\sigma_{\omega} \ldots \ldots \ldots \ldots$

D.7 Test Stand Standard Deviation by $\sigma_{\omega}$ and $\beta \ldots \ldots \ldots \ldots$ 


\section{List of Tables}

4.1 Battery Life (in Hours) . . . . . . . . . . . . . . . . . . . . . . 62

4.2 MINITAB Stage One Analysis . . . . . . . . . . . . . . . . . . . 63

4.3 MINITAB Stage Two Analysis . . . . . . . . . . . . . . . . . . . 63

4.4 Outline of Simulations . . . . . . . . . . . . . . . . . . . . . 64

4.5 Empirical Error Simulation Results: $n=8, r=4 \ldots \ldots 65$

4.6 Simulation Results Power 0.50: $n=8, r=4 \ldots \ldots \ldots 67$

4.7 Simulation Results Power 0.90: $n=8, r=4 \ldots \ldots \ldots$

4.8 Empirical Error Rate Simulation Results: $n=16, r=8 \ldots \ldots 9$

4.9 Simulation Results Power 0.50: $n=16, r=8 \ldots \ldots 70$

4.10 Simulation Results Power 0.90: $n=16, r=8 \ldots \ldots \ldots \ldots 71$

4.11 Empirical Error Rate Simulation Results: $n=16, r=12 \ldots \ldots 72$

4.12 Simulation Results Power 0.50: $n=16, r=12 \ldots \ldots \ldots 73$ 
4.13 Simulation Results Power 0.90: $n=16, r=12 \ldots \ldots 74$

$5.1 \quad$ NLMM Analysis Results . . . . . . . . . . . . . . . . . . . . . . 90

5.2 Estimates and Standard Errors for the Traditional Analysis, Traditional Analysis with blocks, Two-Stage Analysis and NLMM Analysis . . . . . . . . . 91

5.3 Estimates and Confidence intervals for $t_{0.01} \ldots \ldots \ldots \ldots 2$

A.1 Simulation Results Power 0.80: $n=8, r=4 \ldots \ldots \ldots \ldots$

A.2 Simulation Results Power 0.80: $n=16, r=8 \ldots \ldots \ldots \ldots$

A.3 Simulation Results Power 0.80: $n=16, r=12 \ldots \ldots \ldots \ldots$ 


\section{Chapter 1}

\section{Introduction}

Consumers demand high quality products. To remain competitive in today's global market, manufacturers must strive to meet consumer's quality expectations. An increasingly important characteristic is the product's lifetime. Therefore, manufacturers need to thoroughly understand the expected life of their product under various operating conditions. They must also seek new ways to extend product lifetime. Reliability and product lifetime are also important for safety. Department of Defense (DoD) and National Aeronautics and Space Administration (NASA) manned systems require highly reliable systems for our service members and civilians. For example, consider the composite pressure vessels on the Space-Shuttle Orbiter vehicles. Each vehicle contains twenty-two pressure vessels, where the failure of any one pressure vessel could have catastrophic consequences. Thus, scientists at NASA must thoroughly understand the reliability and lifetime of pressure vessels. 
Reliability experiments provide important lifetime information to developers and manufacturers. A well designed reliability experiment allows manufactures to understand what lifetimes can be expected of their products under various operating conditions and also provide information on factors important to designing reliability into a product. However, reliability experiments can also be complex to execute. The manufacturing industry works under everpresent cost and time constraints. These constraints often result in reliability experiments that are not completely randomized and contain censored data.

\subsection{The Weibull Distribution}

Engineers know that in many applications failure times follow the Weibull distribution. The flexibility of the Weibull distribution makes it appropriate for modeling many types of failure mechanisms. The probability density function (PDF) of the Weibull distribution is

$$
f(t \mid \beta, \eta)=\frac{\beta}{\eta}\left(\frac{t}{\eta}\right)^{\beta-1} e^{-\left(\frac{t}{\eta}\right)^{\beta}}
$$

where $t>0$ is the time to failure, $\beta>0$ is the shape parameter, and $\eta>0$ is the scale parameter. Figure 1.1 shows the PDF for the Weibull distribution with a scale parameter $\eta=100$ and several values of the shape parameter. The exponential distribution is a special case of the Weibull distribution when $\beta=1$, and the Weibull distribution closely resembles the normal distribution for $\beta=3$. 
Figure 1.1: Probability Distribution Function of the Weibull Distribution $(\eta=100)$

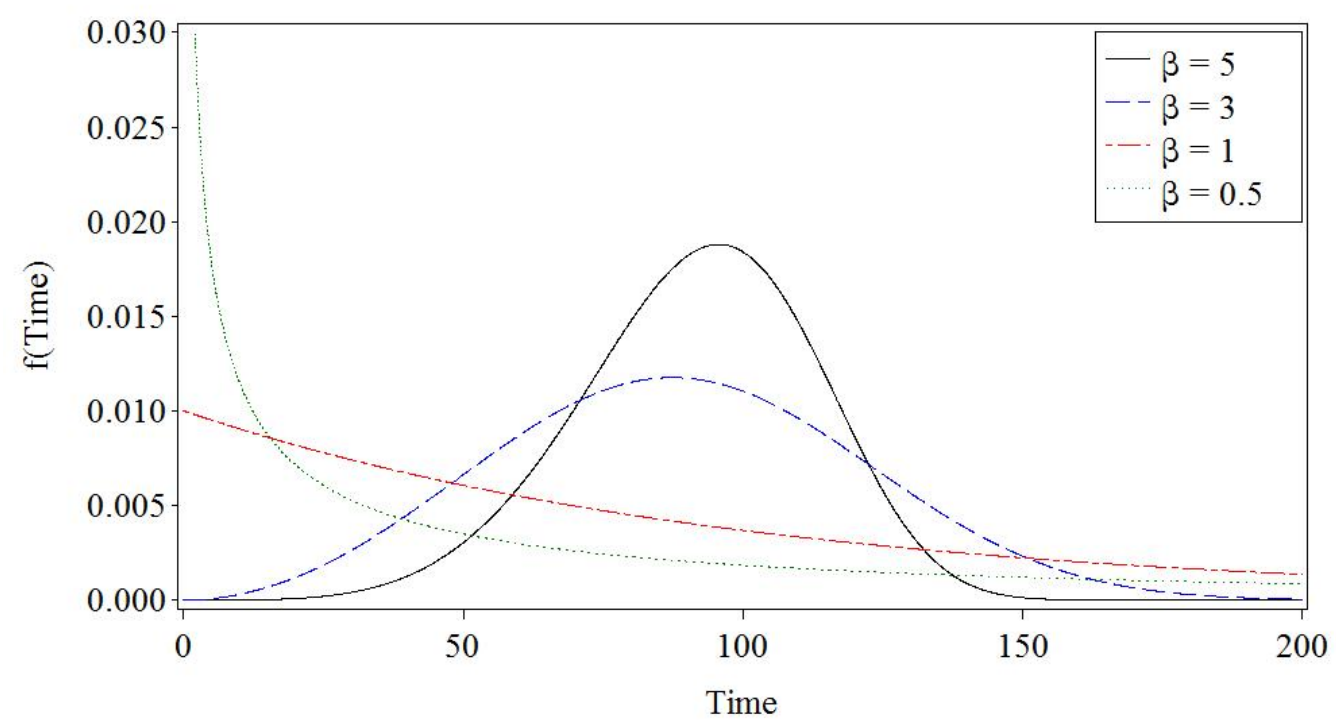

The shape parameter, $\beta$, reflects the failure mechanism and hence the shape of the distribution. The scale parameter (or characteristic life), $\eta$, determines the spread of the distribution and denotes the time by which $63.2 \%$ of units fail.

The cumulative distribution function $(\mathrm{CDF})$,

$$
F(t \mid \beta, \eta)=1-e^{-\left(\frac{t}{\eta}\right)^{\beta}}
$$

gives the probability that a unit fails by time $t$.

The Weibull distribution has a mean of $\eta \Gamma(1+1 / \beta)$ where $\Gamma$ denotes the gamma function, $\Gamma(\kappa)=\int_{0}^{\infty} z^{\kappa-1} e^{-z} d z$, and a variance of $\eta^{2}\left[\Gamma(1+2 / \beta)-\Gamma^{2}(1+1 / \beta)\right]$. Note that the mean and variance are functions of both Weibull parameters $(\beta$ and $\eta)$. 
In reliability analysis the hazard function provides important information regarding the failure mechanism. The hazard function, also known as the instantaneous failure rate, gives the probability of failure in the next small period of time, given that the unit has survived until now. The hazard function for the Weibull distribution is

$$
h(t \mid \beta, \eta)=\left(\frac{\beta}{\eta}\right)\left(\frac{t}{\eta}\right)^{\beta-1} .
$$

Figure 1.2 shows the hazard function for the Weibull distribution for $\eta=100$ and several values of $\beta$.

Figure 1.2: Hazard Function of the Weibull Distribution $(\eta=100)$

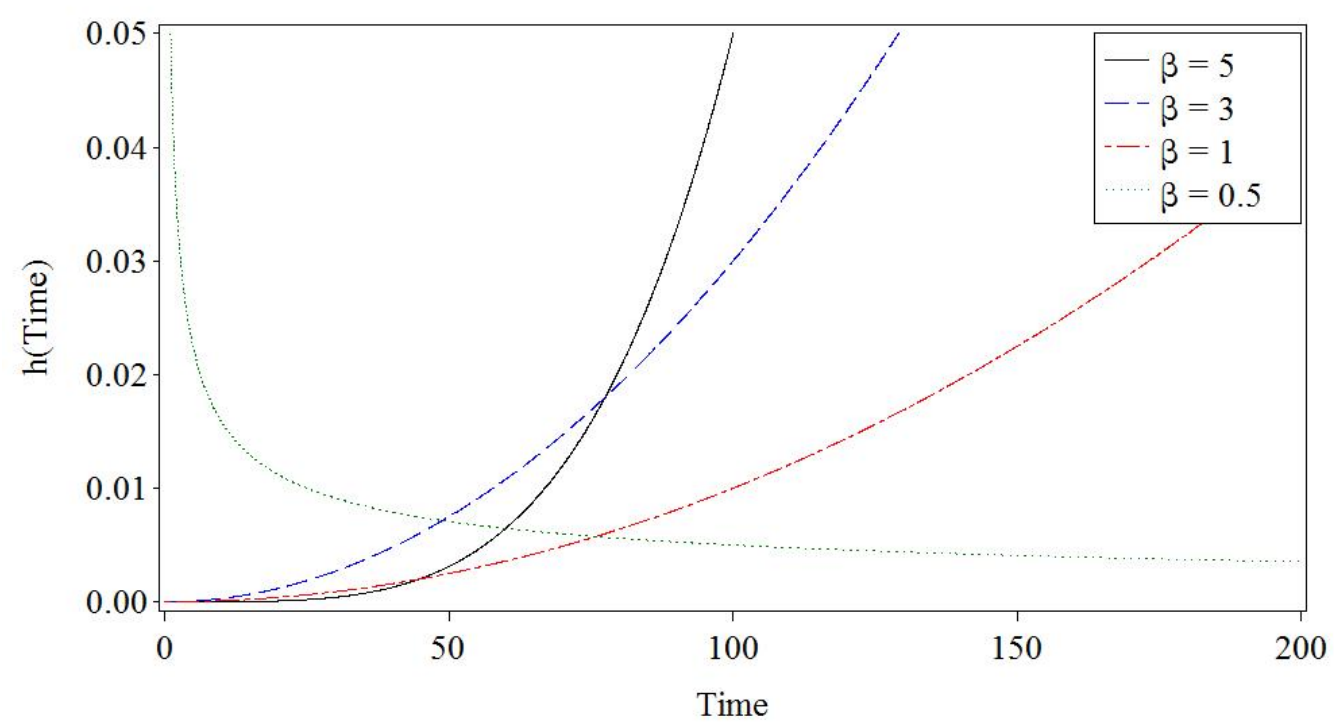

The value of the shape parameter, $\beta$, reflects the underlying failure mechanism. The Weibull distribution has a decreasing hazard function for $\beta<1$, which reflects failure due to infant mortality (early failure of an item possibly due to a defect). For $\beta=1$ the Weibull distribu- 
tion has a constant hazard function, which indicates random failure. The Weibull distribution has an increasing hazard function for $\beta>1$, which indicates failure due to product wear out.

\subsection{Censoring}

In an experiment waiting for all units to fail proves impractical in some cases. Many products are designed to last years or even decades before failure, but manufacturers cannot wait that long to understand the lifetime distributions. Censoring occurs when the experimenter does not observe the exact time of failure. Right censoring occurs when the test stops before all units fail. In type I right censoring, an experiment terminates at a predetermined time. On the other hand, type II right censoring occurs when the experiment stops after a prespecified number of failures. Experiments with type I and type II censoring may also contain interval, and/or left censoring. Interval censoring occurs when one knows a failure occurs between two times but does not know the exact failure time. For example, a unit failed sometime after it was inspected at ten weeks, but before it was inspected at eleven weeks. Left censoring occurs when the failure occurs before a known time (i.e. the unit failed before the first inspection at week one).

Engineers often perform reliability experiments with censoring to determine how various factors affect product life. For example, if engineers believe operating voltage affects the life of a light bulb they may design an experiment with four voltage levels and operate ten light bulbs at each voltage. The experimenters implement type II right censoring in order 
to complete the experiment in a timely fashion, while ensuring a specific number of failures. In this case, the researchers record the times at which the first five light bulbs fail for each voltage level. The remaining five light bulbs represent censored observations.

\subsection{Experimental Design}

The three principles of experimental design are randomization, replication and local control of error. Randomization refers to the random assignment of units to treatments and to the randomization of the order of the performance of the experiment. Randomization helps to prevent bias and the confounding of factors, both known and unknown. Replication means repeating the application of the treatments more than once. The more replications an experiment contains the more information that can be extracted. Replication allows the calculation a pure error estimate of the variance. This pure error estimate of the variance allows testing of factor effects and testing for lack of fit. Local control of error involves accounting for other issues, such as covariates or batch effects. In some cases, for example, complete randomization may not be practical due to time and/or cost constraints. It is important that the analysis reflects the protocol of the experiment actually run with regards to the degree of randomization and replication. 


\subsection{Outline}

This dissertation examines methods for analyzing a reliability experiment where the design contains random blocks and subsampling. This design contains restrictions in randomization that lead to a model with two error terms. This dissertation proposes two methods of analyzing this design when the (possibly right-censored) failure times follow a Weibull distribution.

Chapter 2 provides a review of the literature for reliability and design of experiments. It reviews the reliability methods necessary for this work, including parameter estimation, inference, and regression with life data for log-location-scale models. From the design of experiments literature, an overview of random block designs and subsampling is given. Chapter 2 concludes by outlining two methods for the analysis of a reliability experiment with subsampling, upon which this current dissertation builds.

Chapter 3 presents results from a simulation study that compares methods of analyzing the reliability experiment with subsampling introduced in Chapter 2. These simulations provide insight necessary for simulations performed in later chapters.

Chapter 4 introduces a two-stage method for analyzing a reliability experiment with random blocks and subsampling. This two-stage method is easy to implement and may be performed in popular commercial software such as MINITAB and JMP. Chapter 4 also uses simulations to examine the properties of the two-stage method and compares the two-stage method with traditional reliability analyses. 
Chapter 5 proposes a more rigorous nonlinear mixed model (NLMM) analysis for a reliability experiment with random blocks and subsampling. This chapter also shows how to perform inferences on parameters and functions of parameters (including percentiles).

Chapter 6 uses simulation studies to examine the properties of the more rigorous NLMM solution. The performance of the NLMM solution is compared with that of the two-stage and traditional methods.

Chapter 7 contains concluding remarks and lists ideas for future research. 


\section{Chapter 2}

\section{Literature Review}

This literature review consists of three sections. The first section gives an overview of the topics in reliability necessary to this work. The second section discusses discusses relevant topics from design of experiments. The third section examines the analysis of a reliability experiment with subsampling and outlines the analyses proposed by Freeman and Vining (2010, 2012) and Freeman (2010).

\subsection{Analysis of Lifetime Data}

Meeker and Escobar (1998), Lawless (2003), and Nelson (1990) provide excellent introductions to lifetime data, reliability experiments and their analyses. These references include many important topics in reliability: popular distributions for lifetime data, parameter estimation, inference, regression with lifetime data, and accelerated life tests. In addition, 
Lawless (1983) highlights the achievements in applying statistics to reliability data, as well as suggesting areas of future work. Although Klein and Moeschberger (2003) focuses on survival analysis, with an emphasis on nonparametric and semiparametric methods, it presents concepts important to all lifetime data applications. These works provide the foundation for this review of reliability methods, which uses notation most closely resembling that of Meeker and Escobar (1998).

\subsubsection{Location-Scale and Log-Location-Scale Distributions}

Analysis of reliability data often assumes that the failure times follow a distribution that belongs to the location-scale or log-location-scale families of distributions. The locationscale family includes the normal, exponential, smallest extreme value (SEV), and logistic distributions. A distribution belongs to the location-scale family if its CDF can be written in the form

$$
F(y \mid \mu, \sigma)=\Phi\left(\frac{y-\mu}{\sigma}\right),
$$

where $\Phi$ denotes the CDF for $\mu=0$ and $\sigma=1$ and does not depend on any unknown parameters. The location parameter, $\mu$, may be any real number and the scale parameter, $\sigma$, must be greater than 0 .

The log-location-scale family includes the lognormal, Weibull, and log-logistic distributions. The distribution of failure times, $T$, belongs to the log-location-scale family if 
$Y=\log (T)$ belongs to the location-scale family. This dissertation focuses on the Weibull distribution, where engineers prefer to work with $\beta=\frac{1}{\sigma}$. Hence this work implements parameterizations of models using $\beta$ instead of $\sigma$. Therefore, Equation 2.1 can be reparameterized as

$$
F(y \mid \mu, \beta)=\Phi(\beta(y-\mu)) .
$$

The use of the location-scale and log-location-scale families allow methods of analysis for several distributions to be obtained simultaneously.

\subsubsection{The Weibull and Smallest Extreme Value Distributions}

Engineers often use the Weibull distribution, due to its flexibility, to represent different failure modes. Equation 1.1 gives the traditional parameterization for the PDF of the Weibull distribution. An alternative parameterization of the Weibull distribution uses $\mu=\log (\eta)$ giving

$$
f(t \mid \mu, \beta)=\frac{\beta}{t} \exp \{\beta(\log (t)-\mu)-\exp [\beta(\log (t)-\mu)]\} .
$$

If the random variable $T$ follows the Weibull distribution, then the random variable $Y=$ $\log (T)$ follows the smallest extreme value distribution. The CDF of the smallest extreme value distribution is 


$$
F(y \mid \mu, \beta)=\Phi_{S E V}[\beta(y-\mu)],
$$

where $\Phi_{S E V}(z)=1-\exp [-\exp (z)]$ and the random variable $Z$ follows the standardized smallest extreme value distribution. The PDF of the smallest extreme value distribution is

$$
f(y \mid \mu, \beta)=\beta \phi_{S E V}[\beta(y-\mu)],
$$

where $\phi_{S E V}(z)=\exp [z-\exp (z)]$.

\subsubsection{Maximum Likelihood Estimation}

Advantageous properties of maximum likelihood (ML) estimation make it popular for analyzing reliability experiments. ML estimation easily incorporates censoring into the analysis. In addition, under certain regularity conditions the maximum likelihood estimates (MLEs) are consistent and asymptotically efficient. The Weibull distribution, the focus of this work, meets these regularity conditions (Meeker and Escobar 1998). For a discussion on the consistency, asymptotic efficiency, and the invariance property of MLEs see Casella and Berger (2002). The likelihood reflecting right censoring for a member of the log-location-scale family is

$$
L(\mu, \beta \mid D a t a)=\mathcal{C} \prod_{i=1}^{n}\left\{\frac{\beta}{t_{i}} \phi\left[\beta\left(\log \left(t_{i}\right)-\mu\right)\right]\right\}^{\delta_{i}} \times\left\{1-\Phi\left[\beta\left(\log \left(t_{i}\right)-\mu\right)\right]\right\}^{1-\delta_{i}},
$$


where

$$
\delta_{i}=\left\{\begin{array}{ll}
1 & \text { if ith observation is an exact failure } \\
0 & \text { if ith observation is censored }
\end{array} .\right.
$$

Meeker and Escobar (1998) discuss the impact of the constant $\mathcal{C}$ on the likelihood. The constant $\mathcal{C}$ reflects the type of censoring and sampling scheme. Since $\mathcal{C}$ is a constant that does not depend on $\beta, \mu$ or the data, one can take $\mathcal{C}=1$ without loss of generality for ML estimation.

In the case of the Weibull distribution, the likelihood is

$$
L(\eta, \beta \mid \text { Data })=\prod_{i=1}^{n}\left[\frac{\beta}{\eta}\left(\frac{t_{i}}{\eta}\right)^{\beta-1} e^{-\left(t_{i} / \eta\right)^{\beta}}\right]^{\delta_{i}} \times\left[e^{-\left(t_{i} / \eta\right)^{\beta}}\right]^{1-\delta_{i}}
$$

where

$$
\delta_{i}=\left\{\begin{array}{ll}
1 & \text { if ith observation is an exact failure } \\
0 & \text { if ith observation is censored }
\end{array} .\right.
$$

For the log-location-scale family the log-likelihood is

$$
\mathcal{L}(\mu, \beta \mid \text { Data })=\sum_{i=1}^{n} \delta_{i} \log \left(\frac{\beta}{t_{i}}\right)+\sum_{i=1}^{n} \delta_{i} \log \left[\phi\left(z_{i}\right)\right]+\sum_{i=1}^{n}\left(1-\delta_{i}\right) \log \left[1-\Phi\left(z_{i}\right)\right]
$$


where $z_{i}=\beta\left(\log \left(t_{i}\right)-\mu\right)$.

The log-likelihood for the Weibull distribution is

$$
\mathcal{L}(\eta, \beta \mid \text { Data })=\sum_{i=1}^{n} \delta_{i} \log \left[\frac{\beta}{\eta}\left(\frac{t_{i}}{\eta}\right)^{\beta-1}\right]-\sum_{i=1}^{n}\left(\frac{t_{i}}{\eta}\right)^{\beta} .
$$

The solution to the following system of equations gives the MLEs for the Weibull distribution:

$$
\begin{gathered}
\eta^{\beta}=\frac{1}{r} \sum_{i=1}^{n} t_{i}^{\beta} \\
\frac{\sum_{i=1}^{n} t_{i}^{\beta} \log \left(t_{i}\right)}{\sum_{i=1}^{n} t_{i}^{\beta}}-\frac{1}{\beta}-\frac{1}{r} \sum_{i=1}^{n} \delta_{i} \log \left(t_{i}\right)=0
\end{gathered}
$$

where $r=\sum_{i=1}^{n} \delta_{i}$. As no closed form solution exists, the solution to these equations must be found numerically. In the case of a known $\beta$, there exists a tractable solution for $\eta$ :

$$
\eta=\left(\frac{1}{r} \sum_{i=1}^{n} t_{i}^{\beta}\right)^{\frac{1}{\beta}}
$$

\subsubsection{Bias of Maximum Likelihood Estimates of the Weibull Shape Parameter for Small Sample Sizes}

While the MLEs have good large sample properties (including being consistent and asymptotically efficient), reliability experiments often involve small sample sizes. It has long been recognized that the ML estimation of the Weibull parameters produces biased results for 
small sample sizes. In particular, many investigators have examined the overestimation of $\beta$ on small sample sizes for both complete and censored data. Two recent studies include Olteanu and Freeman (2010) and Genschel and Meeker (2010). While various techniques exist for obtaining unbiased estimates for $\beta$ (including modified likelihoods and linear regression), this section examines bias correction factors that practitioners can easily implement using software such as MINITAB and JMP. The product of a bias correction factor and the MLE of $\beta, \hat{\beta}$, produces an unbiased estimate of $\beta$. See Montanari et al. (1997) for a comparison of techniques to obtain an unbiased estimate of $\beta$.

Thoman et al. (1969) examines the bias of $\hat{\beta}$ for complete data. Simulations involving the pivotal function $\hat{\beta} / \beta$ are used to create a table of bias correction factors. Thoman et al. (1969) notes that the bias of $\hat{\beta}$ is independent of $\beta$ and $\eta$ and depends only on the sample size.

Billmann et al. (1972) investigates the bias of $\hat{\beta}$ for cases of $25 \%$ and $50 \%$ censoring. For censored data, the bias of $\hat{\beta}$ depends only on the sample size and the number of failures. Billmann et al. (1972) uses simulations involving the pivotal function $\hat{\beta} / \beta$ to create a table of bias correction factors.

Ross (1994) introduces a formula for a bias correction factor for the shape parameter in the case of complete data with sample size $n$. The bias correction factor is $\frac{n-2}{n-0.68}$, which produces unbiasing values consistent with those suggested by Thoman et al. (1969). Ross (1996) extends this work to the case of censored data with sample size $n$ and $r$ failures. A bias correction factor for data with $50 \%$ or less censoring is 


$$
\frac{1}{1+\frac{1.37}{r-1.92} \sqrt{\frac{n}{r}}} \text {. }
$$

Cacciari et al. (1996) compares several methods of obtaining unbiased estimates of the Weibull parameters. For the case of complete data, a modification of Ross (1994): ( $n-$ $1.93) /(n-0.6)$ is recommended. However, Cacciari et al. (1996) notes that this bias correction factor is for the expected value of $\beta$. The recommended bias correction factor for a point estimate of $\beta$ is

$$
\left[\frac{g / h+2 / g}{3 g /(h-0.7)-2 g / h-4 / g}\right]\left[\frac{n-2}{n-0.68}\right],
$$

where $g=h+2$ and Lawless (2003) provides a table for the values of $h$. The constants $g$ and $h$ depend on the sample size and number of failures. An approximation for the distribution of $\frac{\hat{\beta}}{\beta}$ is $g\left(\frac{\hat{\beta}}{\beta}\right) \sim \chi_{h}^{2}$.

Freeman (2011) examines the bias of the shape parameter for grouped data. Simulations with $50 \%$ censoring show that type I censoring and complete data produce similar biases for the shape parameter. However, type II censoring causes a more severe overestimation of $\beta$. Freeman (2011) recommends sample sizes of at least 20 to 40 (depending on the type of censoring) for the asymptotic properties of the MLEs to take effect.

Bias correction factors for the MLE of the Weibull shape parameter have been explored for the cases of complete and censored data. However, the literature does not appear to suggest 
a bias correction factor for the case of grouped data.

\subsubsection{Inference Based on the Likelihood}

Inferences of interest include the parameters $(\mu, \beta), t_{p}$ and $F(t)$. The quantity $t_{p}$ (the $p^{t h}$ quantile) represents the expected time by which $100 p \%$ of units have failed. Similarly, $F\left(t_{e}\right)$ denotes the probability that an item fails by time $t_{e}$. Popular methods for obtaining inferences on these quantities include likelihood based methods and methods based on approximations to the normal distribution. Likelihood based inferences provide more accurate coverage probabilities, but require the more difficult to obtain profile likelihoods. Inferences based on approximations to the normal distribution are easier to obtain, but are less accurate for small sample sizes. This section examines inferences based on the likelihood.

The region

$$
R(\mu, \beta)>\exp \left(-\chi_{1-\alpha, 2}^{2} / 2\right)=\alpha
$$

gives a $100(1-\alpha) \%$ joint confidence interval for $\mu$ and $\beta$, where

$$
R(\mu, \beta)=\frac{L(\mu, \beta)}{L(\hat{\mu}, \hat{\beta})}
$$

denotes the relative likelihood function. 
The use of profile likelihoods allow the calculation of individual confidence intervals for the parameters $\mu$ and $\beta$. The interval where $R(\mu)>\exp \left[-\chi_{(1-\alpha, 1)}^{2} / 2\right]$ provides a $100(1-\alpha) \%$ confidence interval for $\mu$, where

$$
R(\mu)=\max _{\beta}\left[\frac{L(\mu, \beta)}{L(\hat{\mu}, \hat{\beta})}\right]
$$

represents the profile likelihood for $\mu$.

Likewise, the interval where $R(\beta)>\exp \left[-\chi_{(1-\alpha, 1)}^{2} / 2\right]$ produces a $100(1-\alpha) \%$ confidence interval for $\beta$. Note that the profile likelihood for $\beta$ is

$$
R(\beta)=\max _{\mu}\left[\frac{L(\mu, \beta)}{L(\hat{\mu}, \hat{\beta})}\right] .
$$

In reliability analysis the $p^{\text {th }}$ quantile, the expected time by which $100 \mathrm{p} \%$ of the items have failed, provides important information. For members of the log-location-scale family, the MLE of the $p^{t h}$ quantile is

$$
\hat{t}_{p}=\exp \left[\hat{\mu}+\frac{\Phi^{-1}(p)}{\hat{\beta}}\right]
$$

The profile likelihood for $t_{p}$ is

$$
R\left(t_{p}\right)=\max _{\beta}\left[\frac{L\left(t_{p}, \beta\right)}{L(\hat{\mu}, \hat{\beta})}\right]
$$


where $\mu=\log \left(t_{p}\right)-\frac{\Phi^{-1}(p)}{\beta}$ is substituted into $L(\mu, \beta)$ to obtain the likelihood $L\left(t_{p}, \beta\right)$. The interval where $R\left(t_{p}\right)>\exp \left[-\chi_{(1-\alpha, 1)}^{2} / 2\right]$ gives an $100(1-\alpha) \%$ confidence interval for $t_{p}$.

The proportion of items failing by time $t_{e}, F\left(t_{e}\right)$, also provides important information about the lifetime distribution. The interval where $R\left(F\left(t_{e}\right)\right)>\exp \left[-\chi_{(1-\alpha, 1)}^{2} / 2\right]$ provides an $100(1-$ $\alpha) \%$ confidence interval for $F\left(t_{e}\right)$, where

$$
R\left(F\left(t_{e}\right)\right)=\max _{\beta}\left[\frac{L\left(F\left(t_{e}\right), \beta\right)}{L(\hat{\mu}, \hat{\beta})}\right]
$$

denotes the profile likelihood of $F\left(t_{e}\right)$. Substituting $\mu=\log \left(t_{e}\right)-\frac{\Phi^{-1}\left(F\left(t_{e}\right)\right)}{\beta}$ into $L(\mu, \beta)$ gives the likelihood $L\left(F\left(t_{e}\right), \beta\right)$.

\subsubsection{Inference Based on Normal Theory}

Inferences based on normal theory require the estimation of the variance-covariance matrix of MLEs. The variance-covariance matrix is estimated using the inverse of the observed information matrix: 


$$
\begin{aligned}
\hat{\Sigma} & =\left[\begin{array}{cc}
\widehat{\operatorname{Var}}(\hat{\mu}) & \widehat{\operatorname{Cov}}(\hat{\mu}, \hat{\beta}) \\
\widehat{\operatorname{Cov}}(\hat{\mu}, \hat{\beta}) & \widehat{\operatorname{Var}}(\hat{\beta})
\end{array}\right] \\
& =\left[\begin{array}{cc}
-\frac{\partial^{2} \mathcal{L}(\hat{\mu}, \hat{\beta})}{\partial \mu^{2}} & -\frac{\partial^{2} \mathcal{L}(\hat{\mu}, \hat{\beta})}{\partial \mu \partial \beta} \\
-\frac{\partial^{2} \mathcal{L}(\hat{\mu}, \hat{\beta})}{\partial \beta \partial \mu} & -\frac{\partial^{2} \mathcal{L}(\hat{\mu}, \hat{\beta})}{\partial \beta^{2}}
\end{array}\right]^{-1} .
\end{aligned}
$$

In the case of the Weibull distribution the second order partial derivatives are $\frac{\partial^{2}}{\partial \mu^{2}} \mathcal{L}(\mu, \beta)=$ $-\beta^{2} \sum_{i=1}^{n}\left(t_{i} e^{-\mu}\right)^{\beta}, \frac{\partial^{2}}{\partial \mu \partial \beta} \mathcal{L}(\mu, \beta)=\sum_{i=1}^{n}\left[-\delta_{i}+e^{z_{i}}\left(z_{i}+1\right)\right]$, and $\frac{\partial^{2}}{\partial \beta^{2}} \mathcal{L}(\mu, \beta)=\sum_{i=1}^{n}\left[-\frac{\delta_{i}}{\beta}+\frac{z_{i}}{\beta}\left(\delta_{i}-e^{z_{i}}\right)\right]$ with $z_{i}=\beta\left(\log \left(t_{i}\right)-\mu\right)$.

A $100(1-\alpha) \%$ confidence interval for $\mu$ is

$$
\hat{\mu} \pm z_{1-\alpha / 2} \widehat{s e}_{\hat{\mu}}
$$

where $\widehat{s e} \hat{\mu}=\sqrt{\widehat{\operatorname{Var}}(\hat{\mu})}$. In a similar manner a $100(1-\alpha) \%$ confidence interval for $\beta$ is

$$
\hat{\beta} \pm z_{1-\alpha / 2} \widehat{s e}_{\hat{\beta}}
$$

where $\widehat{s e}_{\hat{\beta}}=\sqrt{\widehat{\operatorname{Var}}(\hat{\beta})}$. Since $\beta>0$, it is common to use a log transformation to compute a confidence interval for $\beta$ (i.e. the distribution of $\frac{\log (\hat{\beta})-\log (\beta)}{\hat{s} \hat{\log }_{(\hat{\beta})}}$ is approximately $N(0,1)$ ). In this case, the interval 


$$
\left(\frac{\hat{\beta}}{w}, \hat{\beta} \times w\right)
$$

produces a $100(1-\alpha) \%$ confidence interval for $\beta$, where $w=\exp \left[z_{1-\alpha / 2} \widehat{s e}_{\hat{\beta}} / \hat{\beta}\right]$ and $\widehat{s e}_{\hat{\beta}}=$ $\sqrt{\widehat{\operatorname{Var}}(\hat{\beta})}$.

For $t_{p}$ the interval

$$
\left(\frac{\hat{t}_{p}}{w}, \hat{t}_{p} \times w\right)
$$

gives a $100(1-\alpha) \%$ confidence interval, where $w=\exp \left[z_{1-\alpha / 2} \widehat{s e}_{\hat{t}_{p}} / \hat{t}_{p}\right]$ and

$$
\widehat{s e}_{\hat{t}_{p}}=\hat{t}_{p}\left\{\widehat{\operatorname{Var}}(\hat{\mu})-2 \frac{\Phi^{-1}(p)}{\hat{\beta}^{2}} \widehat{\operatorname{Cov}}(\hat{\mu}, \hat{\beta})+\left(\frac{\Phi^{-1}(p)}{\hat{\beta}^{2}}\right)^{2} \widehat{\operatorname{Var}}(\hat{\beta})\right\}^{1 / 2} .
$$

This interval is based on the distribution of $\frac{\log \left(\hat{t}_{p}\right)-\log \left(t_{p}\right)}{\widehat{s}_{\log \left(\hat{t}_{p}\right)}}$ being approximately $N(0,1)$.

One confidence interval for $F\left(t_{e}\right)$ is

$$
\hat{F}\left(t_{e}\right) \pm z_{1-\alpha / 2} \widehat{s e}_{\hat{F}}
$$

where

$$
\widehat{s e}_{\hat{F}}=\phi\left(\hat{z}_{e}\right)\left\{\hat{\beta}^{2} \widehat{\operatorname{Var}}(\hat{\mu})-2 z_{e} \widehat{\operatorname{Cov}}(\hat{\mu}, \hat{\beta})+\frac{z_{e}^{2}}{\hat{\beta}^{2}} \widehat{\operatorname{Var}}(\hat{\beta})\right\}^{1 / 2}
$$


and $\hat{z}_{e}=\beta\left(\log \left(t_{e}\right)-\hat{\mu}\right)$. Since $F(t)$ must be between 0 and 1 , an alternative confidence interval, which is considered more accurate, is based on the logit transformation where $\operatorname{logit}(F)=\log \left(\frac{F}{1-F}\right)$. Assuming the distribution of $\frac{\operatorname{logit}(\hat{F})-\operatorname{logit}(F)}{\hat{s} e_{\operatorname{logit}}(\hat{F})}$ is approximately standard normal leads to the $100(1-\alpha) \%$ confidence interval

$$
\left(\frac{\hat{F}}{\hat{F}+(1-\hat{F}) \times w}, \frac{\hat{F}}{\hat{F}+(1-\hat{F}) / w}\right) \text {, }
$$

where $w=\exp \left\{\left(z_{1-\alpha / 2} \widehat{s e}_{\hat{F}}\right) /[\hat{F}(1-\hat{F})]\right\}$. Hong et al. (2008a) recommends a confidence interval for $F(t)$ based on the quantile transformation function, $\Phi^{-1}$.

\subsubsection{Regression with Lifetime Data}

Regression methods allow the incorporation of explanatory variables into the model. In traditional regression models, such as generalized linear models, explanatory variables typically enter the model through the mean. However, in the case of the log-location-scale family the mean may not have a closed form. Instead, explanatory variables commonly enter the model through the parameter $\mu$ or less often through the parameter $\beta$. In addition, if an explanatory variable impacts the lifetime of a product it makes sense for it to have a relationship with $\mu$, since $\mu$ is the log of the characteristic life. The work in this dissertation focuses on independent variables entering the model through the parameter $\mu$ such that 


$$
\mu_{i}=\mathbf{x}_{i}^{T} \gamma
$$

In the case of simple linear regression

$$
\mu_{i}=\gamma_{0}+\gamma_{1} x_{i} .
$$

The likelihood reflecting right censoring for members of the log-location scale family is

$$
L\left(\gamma_{0}, \gamma_{1}, \beta \mid D a t a\right)=\mathcal{C} \prod_{i=1}^{n}\left\{\frac{\beta}{t_{i}} \phi\left[\beta\left(\log \left(t_{i}\right)-\mu_{i}\right)\right]\right\}^{\delta_{i}}\left\{1-\Phi\left[\beta\left(\log \left(t_{i}\right)-\mu_{i}\right)\right]\right\}^{1-\delta_{i}}
$$

where $\mu_{i}=\gamma_{0}+\gamma_{1} x_{i}$. Note that $\mathcal{C}$ is a constant and may be taken as 1 without loss of generality for ML estimation.

The variance-covariance matrix of the MLEs is estimated by taking the inverse of the observed information matrix 


$$
\begin{aligned}
\hat{\Sigma} & =\left[\begin{array}{ccc}
\widehat{\operatorname{Var}}\left(\hat{\gamma}_{0}\right) & \widehat{\operatorname{Cov}}\left(\hat{\gamma}_{0}, \hat{\gamma}_{1}\right) & \widehat{\operatorname{Cov}}\left(\hat{\gamma}_{0}, \hat{\beta}\right) \\
\widehat{\operatorname{Cov}}\left(\hat{\gamma}_{1}, \hat{\gamma}_{0}\right) & \widehat{\operatorname{Var}}\left(\hat{\gamma}_{1}\right) & \widehat{\operatorname{Cov}}\left(\hat{\gamma}_{1}, \hat{\beta}\right) \\
\widehat{\operatorname{Cov}}\left(\hat{\beta}_{1}, \hat{\gamma}_{0}\right) & \widehat{\operatorname{Cov}}\left(\hat{\beta}, \hat{\gamma}_{1}\right) & \widehat{\operatorname{Var}}(\hat{\beta})
\end{array}\right] \\
& =\left[\begin{array}{ccc}
-\frac{\partial^{2} \mathcal{L}\left(\hat{\gamma}_{0}, \hat{\gamma}_{1}, \hat{\beta}\right)}{\partial \gamma_{0}^{2}} & -\frac{\partial^{2} \mathcal{L}\left(\hat{\gamma}_{0}, \hat{\gamma}_{1}, \hat{\beta}\right)}{\partial \gamma_{0} \gamma_{1}} & -\frac{\partial^{2} \mathcal{L}\left(\hat{\gamma}_{0}, \hat{\gamma}_{1}, \hat{\beta}\right)}{\partial \gamma_{0} \partial \beta} \\
-\frac{\partial^{2} \mathcal{L}\left(\hat{\gamma}_{0}, \hat{\gamma}_{1}, \hat{\beta}\right)}{\partial \gamma_{1} \partial \gamma_{0}} & -\frac{\partial^{2} \mathcal{L}\left(\hat{\gamma}_{0}, \hat{\gamma}_{1}, \hat{\beta}\right)}{\partial \gamma_{1}^{2}} & -\frac{\partial^{2} \mathcal{L}\left(\hat{\gamma}_{0}, \hat{\gamma}_{1}, \hat{\beta}\right)}{\partial \gamma_{1} \partial \beta} \\
-\frac{\left.\partial^{2} \mathcal{L} \mathcal{\gamma _ { 0 }}, \hat{\gamma}_{1}, \hat{\beta}\right)}{\partial \beta \partial \gamma_{0}} & -\frac{\partial^{2} \mathcal{L}\left(\hat{\gamma}_{0}, \hat{\gamma}_{1}, \hat{\beta}\right)}{\partial \beta \partial \gamma_{1}} & -\frac{\partial^{2} \mathcal{L}\left(\hat{\gamma}_{0}, \hat{\gamma}_{1}, \hat{\beta}\right)}{\partial \beta^{2}}
\end{array}\right]^{-1}
\end{aligned}
$$

Confidence intervals for the parameters $\gamma_{0}, \gamma_{1}$ and $\beta$ and functions of these parameters are constructed from elements of this variance-covariance matrix. In particular, making inferences about $\mu$ is of interest. Note that

$$
\widehat{\operatorname{Var}}(\hat{\mu})=\widehat{\operatorname{Var}}\left(\hat{\gamma}_{0}\right)+2 x \widehat{\operatorname{Cov}}\left(\hat{\gamma}_{0}, \hat{\gamma}_{1}\right)+x^{2} \widehat{\operatorname{Var}}\left(\gamma_{1}\right)
$$

and

$$
\widehat{\operatorname{Cov}}(\hat{\mu}, \hat{\beta})=\widehat{\operatorname{Cov}}\left(\hat{\gamma}_{0}, \hat{\beta}\right)+x \widehat{\operatorname{Cov}}\left(\hat{\gamma}_{1}, \hat{\beta}\right) .
$$

These properties are also used to construct confidence intervals and tests for $t_{p}$ and $F\left(t_{e}\right)$. 


\subsection{Design of Experiments}

Analysis of reliability data traditionally ignores restrictions on randomization in the experiment. Although Hamada (1995) does in fact discuss the importance of using designed experiments in reliability, the examples involve only completely randomized designs. In contrast, Montgomery (2005) and Kutner et al. (2005) provide wonderful introductions to design of experiments, focusing primarily on normally distributed data. This dissertation proposes two analyses that incorporate the experimental design and protocol into the analysis for reliability experiments with random blocks and subsampling. This section presents a brief overview of subsampling and random blocks.

\subsubsection{Subsampling}

Subsampling occurs when more than one observational unit per experimental unit exists. The experimental unit is the unit to which the treatment is applied. The observational unit is the unit that is measured. Consider an experiment in which a researcher wants to determine the effect of different diets on fish. The researcher has ten fish tanks each with five fish. Five of the tanks receive one type of food and the other five tanks another type of food. The researcher measures the weight of each fish. In this example, the fish are the observational units and the tanks are the experimental units.

One must account for subsampling in the analysis of an experiment. Treating observational units as experimental units causes the experimental error to be underestimated and the 
significance of treatment effects to be overstated. Subsampling is related to the principle of replication in experimental design. The analysis must account for the fact that observational units do not represent true replicates.

The model for one factor with subsampling is

$$
\begin{aligned}
i & =1,2, \ldots, t \\
y_{i j k}=\mu+\tau_{i}+\varepsilon_{j(i)}+\delta_{i j k}, \quad j & =1,2, \ldots, n \\
k & =1,2, \ldots, m
\end{aligned}
$$

where $\tau_{i}$ is the $i^{\text {th }}$ treatment effect, $\varepsilon_{j(i)}$ is the experimental error for an experimental unit and $\delta_{i j k}$ is the error associated with the $k^{\text {th }}$ subsample of an experimental unit. In addition, $\varepsilon_{j(i)} \sim \operatorname{iid} N\left(0, \sigma^{2}\right), \delta_{i j k} \sim \operatorname{iid} N\left(0, \sigma_{\delta}^{2}\right)$, and $\varepsilon_{j(i)}$ and $\delta_{i j k}$ are independent.

\subsubsection{Random Blocks}

Blocking is an instance of local control of error. As an example of blocking, suppose a researcher applies various treatments to individual batteries and measures the performance of each battery. The batteries are manufactured in batches; however, one batch does not produce enough batteries for the entire experiment. Thus, more than one batch is used. The researcher needs to account for variation among batches, which in this case represent blocks. Incorporating the block effect into the analysis increases the precision in estimating the treatment effect. In this example the batch effect represents a random block effect (as 
opposed to a fixed block effect) because we are not concerned with the particular batches, but rather with the variability that batches introduce into the performance of the batteries. The model for a single factor with a random block effect is

$$
y_{i j}=\mu+\tau_{i}+\beta_{j}+\varepsilon_{i j}, \quad \begin{aligned}
& i=1,2, \ldots, t \\
& j=1,2, \ldots, b
\end{aligned}
$$

where $\tau_{i}$ is the $i^{t h}$ treatment effect, $\beta_{j} \sim N\left(0, \sigma_{\beta}^{2}\right)$ is the $j^{\text {th }}$ random block effect, $\varepsilon_{i j} \sim$ $N\left(0, \sigma^{2}\right)$ is the experimental error, and $\varepsilon_{i j}$ and $\beta_{j}$ are independent.

The necessity of accounting for random block effects in reliability experiments has caused much discussion. Gerstle and Kunz (1983) describes an experiment which has been used to underline the importance of incorporating batch effects into the model. In the experiment, scientists at the Lawrence Livermore National Laboratories wrapped composite pressure vessels with Kevlar 49 epoxy. The Kevlar 49 epoxy strands came from eight different spools which represent batches. Scientists pressurized each vessel to one of four pressure levels and recorded the failure time or censoring time. Gerstle and Kunz (1983) notes significant spool-to-spool variation in failure times. Feiveson and Kulkarni (2000) gives an example explaining why spool-to-spool variation should be treated as a random block effect. The example scrutinizes the performance of space-shuttle orbiters, each of which contains 22 pressure vessels. Failure of any one pressure vessel causes the orbiter itself to fail. In this example, records were not kept indicating which spool the pressure vessels were wound from 
(one orbiter may have vessels made from multiple spools). Thus, treating spool variation as a random effect is necessary. Leon et al. (2009) uses simulations based on the pressure vessel data to show that ignoring random batch effects leads one to overestimate the precision.

\subsection{Reliability Experiments with Subsampling}

Freeman and Vining $(2010,2012)$ propose two methods for analyzing reliability experiments containing subsampling. Traditionally, subsampling in reliability experiments has been ignored and the observational units treated as experimental units. This results in misleadingly narrow confidence intervals and may lead the researcher to overstate the significance of factors. Freeman and Vining (2010) proposes a two-stage method which can easily be implemented with standard statistical software packages. However, this method fails to jointly estimate the variances of the shape parameters and the factors. Thus, Freeman and Vining (2012) proposes a second more rigorous method.

\subsubsection{Two-Stage Method for Subsampling}

Zelen (1959) provides an example of a reliability experiment containing subsampling. The experiment involves determining the effects of voltage and temperature on the life of a glass capacitor. The experiment has four voltage levels and two temperature levels. For each combination of the two factors, eight glass capacitors are placed on a single test stand to which the treatment is applied. The experiment utilized type II censoring where the first 
four failures on a test stand were recorded. Note that each combination of factors is only applied to a single test stand. Thus the test stand is the experimental unit and the glass capacitor is the observational unit. Freeman and Vining (2010) notes that the traditional analysis of this data, as in Meeker and Escobar (1998), treats the glass capacitors as if they were the experimental units. Freeman and Vining (2010) introduces a two-stage method and compares the results of this two-stage method with those of the traditional analysis.

If $t_{j(i)}$, the failure time of the $j^{\text {th }}$ item on the $i^{\text {th }}$ test stand, follows a Weibull distribution, then

$$
f\left(t_{j(i)} \mid \eta_{i}, \beta\right)=\frac{\beta}{\eta_{i}}\left(\frac{t_{j(i)}}{\eta_{i}}\right)^{\beta-1} e^{-\left(\frac{t_{j(i)}}{\eta_{i}}\right)^{\beta}}
$$

where $\eta_{i}$ is the scale parameter for the $i^{\text {th }}$ test stand and $\beta$ is the shape parameter. The likelihood for an experiment with right censoring containing $m$ test stands and $n$ items per test stand is

$$
L\left(\beta, \mu_{1}, \ldots, \mu_{m} \mid \text { Data }\right)=\mathcal{C} \prod_{i=1}^{m} \prod_{j=1}^{n}\left[f\left(t_{j(i)}\right)\right]^{\delta_{j(i)}}\left[1-F\left(t_{j(i)}\right)\right]^{1-\delta_{i j}}
$$

where $\mathcal{C}$ is a constant that can be taken to be 1 for ML estimation, $\delta_{j(i)}=1$ if a failure is observed, and $\delta_{j(i)}=0$ if the observation is censored. The log-likelihood is

$$
\mathcal{L}\left(\beta, \mu_{1}, \ldots, \mu_{m} \mid \text { Data }\right)=\sum_{i=1}^{m} \sum_{j=1}^{n}\left(\delta_{j(i)} \log \left(\frac{\beta}{t_{j(i)}}\right)+\delta_{j(i)} z_{j(i)}-e^{z_{j(i)}}\right),
$$


where $z_{j(i)}=\beta\left(\log \left(t_{j(i)}\right)-\mu_{i}\right)$ and $\mu_{i}=\log \left(\eta_{i}\right)=\mathbf{x}_{i}^{T} \gamma+\omega_{i}$. The vector $\mathbf{x}$ contains the factors that affect the scale parameter. Stage 1 uses maximum likelihood to estimate the shape parameter, $\beta$, and $\log$-scale parameters, $\mu_{i}$.

The variance-covariance matrix is estimated using the inverse of the observed information matrix

$$
\begin{aligned}
\hat{\Sigma} & =\left[\begin{array}{cccc}
\widehat{\operatorname{Var}}(\hat{\beta}) & \widehat{\operatorname{Cov}}\left(\hat{\beta}, \hat{\mu}_{1}\right) & \ldots & \widehat{\operatorname{Cov}}\left(\hat{\beta}, \hat{\mu}_{m}\right) \\
\widehat{\operatorname{Cov}}\left(\hat{\beta}, \hat{\mu}_{1}\right) & \widehat{\operatorname{Var}}\left(\hat{\mu}_{1}\right) & & \vdots \\
\vdots & & \ddots & \widehat{\operatorname{Cov}}\left(\hat{\mu}_{m-1}, \hat{\mu}_{m}\right) \\
\widehat{\operatorname{Cov}}\left(\hat{\beta}, \hat{\mu}_{m}\right) & \ldots & \widehat{\operatorname{Cov}}\left(\hat{\mu}_{m-1}, \hat{\mu}_{m}\right) & \widehat{\operatorname{Var}}\left(\hat{\mu}_{m}\right)
\end{array}\right] \\
& =\left[\begin{array}{cccc}
-\frac{\partial^{2} \mathcal{L}\left(\hat{\beta}, \hat{\mu}_{1}, \ldots, \hat{\mu}_{m}\right)}{\partial \beta^{2}} & -\frac{\partial^{2} \mathcal{L}\left(\hat{\beta}, \hat{\mu}_{1}, \ldots, \hat{\mu}_{m}\right)}{\partial \beta \partial \mu_{1}} & \ldots & -\frac{\partial^{2} \mathcal{L}\left(\hat{\beta}, \hat{\mu}_{1}, \ldots, \hat{\mu}_{m}\right)}{\partial \beta \partial \mu_{m}} \\
-\frac{\partial^{2} \mathcal{L}\left(\hat{\beta}, \hat{\mu}_{1}, \ldots, \hat{\mu}_{m}\right)}{\partial \beta \partial \mu_{1}} & -\frac{\partial^{2} \mathcal{L}\left(\hat{\beta}, \hat{\mu}_{1}, \ldots, \hat{\mu}_{m}\right)}{\partial \mu_{1}^{2}} & & \vdots \\
\vdots & & & \vdots \\
-\frac{\partial^{2} \mathcal{L}\left(\hat{\beta}, \hat{\mu}_{1}, \ldots, \hat{\mu}_{m}\right)}{\partial \beta \partial \mu_{m}} & \ldots & -\frac{\partial^{2} \mathcal{L}\left(\hat{\beta}, \hat{\mu}_{1}, \ldots, \hat{\mu}_{m}\right)}{\partial \mu_{m}-1 \partial \mu_{m}} & -\frac{\partial^{2} \mathcal{L}\left(\hat{\beta}, \hat{\beta}_{1}, \ldots, \hat{\mu}_{m}\right)}{\partial \mu_{m}^{2}}
\end{array}\right]^{-1}
\end{aligned}
$$

Note that $-\frac{\partial^{2} \mathcal{L}\left(\hat{\beta}, \hat{\mu}_{1}, \ldots, \hat{\mu}_{m}\right)}{\partial \beta^{2}}=\sum_{i=1}^{m} \sum_{j=1}^{n}\left[\frac{\delta_{j(i)}}{\hat{\beta}^{2}}+\left(\frac{z_{j(i)}}{\hat{\beta}}\right)^{2} \exp \left(\hat{z}_{j(i)}\right)\right],-\frac{\partial^{2} \mathcal{L}\left(\hat{\beta}, \hat{\mu}_{1}, \ldots, \hat{\mu}_{m}\right)}{\partial \mu_{i}^{2}}=\sum_{j=1}^{n} \hat{\beta}^{2} \exp \left(\hat{z}_{j(i)}\right)$, $-\frac{\partial^{2} \mathcal{L}\left(\hat{\beta}, \hat{\mu}_{1}, \ldots, \hat{\mu}_{m}\right)}{\partial \beta \partial \mu_{i}}=\sum_{j=1}^{n}\left[\delta_{j(i)}-\left(\hat{z}_{j(i)}+1\right) \exp \left(\hat{z}_{j(i)}\right)\right]$ and the second partial derivatives between all pairs of $\hat{\mu}_{i}$ and $\hat{\mu}_{i^{\prime}}$ is zero for all $i \neq i^{\prime}$.

Once $\beta$ and the $\mu_{i}$ have been estimated in stage 1 , stage 2 estimates the factor effects, $\gamma$, and test stand error. The stage 2 model is 


$$
\hat{\mu}=\mathbf{X} \gamma+\boldsymbol{\omega}
$$

where $\boldsymbol{\omega} \sim M V N(\mathbf{0}, \mathbf{V})$.

The lack of a joint likelihood incorporating the the shape parameter, $\beta$, and the treatment effects remains a shortcoming of the two-stage method. This lack of a joint likelihood means that inferences cannot be made for functions of the parameters such as $t_{p}$ and $F\left(t_{e}\right)$ that provide vital information about the life distribution. In order to address this issue, Freeman and Vining (2012) proposes a more rigorous NLMM method for analyzing reliability data with subsampling. While this new method allows inferences to be made on functions of parameters, it requires programming using software such as SAS or R.

The joint likelihood for data without censoring is

$$
L\left(\boldsymbol{\gamma}, \beta, \sigma_{\omega} \mid \text { Data }\right)=\int \prod_{i j} f_{1}\left(t_{j(i)} \mid \omega_{i}\right) f_{2}\left(\omega_{i}\right) d \omega_{i},
$$

where $f_{1}\left(t_{j(i)} \mid \omega_{i}\right)$ is the PDF for the conditional distribution of failures for a particular experimental unit $(i=1, \ldots, m)$ and $f_{2}\left(\omega_{i}\right)$ is the PDF for the distribution of observational units $(j=1, \ldots, n)$ within an experimental unit. Freeman and Vining (2012) examines the case where the failures within an experimental unit follow a Weibull distribution, and the test stand effect follows a normal distribution. This gives 


$$
\begin{aligned}
f_{1}\left(t_{j(i)} \mid \beta, \eta_{i}, \omega_{i}\right) & =\frac{\beta}{\eta_{i}}\left(\frac{t_{j(i)}}{\eta_{i}}\right)^{\beta-1} e^{-\left(\frac{t_{j(i)}}{\eta_{i}}\right)^{\beta}} \\
F_{1}\left(t_{j(i)} \mid \beta, \eta_{i}, \omega_{i}\right) & =1-\exp \left[-\left(\frac{t_{j(i)}}{\eta_{i}}\right)^{\beta}\right] \\
\log \left(\eta_{i}\right) & =\mu_{i}=\mathbf{x}_{i}^{T} \boldsymbol{\gamma}+\omega_{i} \\
f_{2}\left(\omega_{i}\right) & =\frac{1}{\sqrt{2 \pi \sigma_{\omega}^{2}}} e^{-\frac{\omega_{i}^{2}}{2 \sigma_{\omega}^{2}}}
\end{aligned}
$$

where $\mathbf{x}_{i}^{T}$ denotes the factor levels and $\boldsymbol{\gamma}$ denotes the factor effects.

The joint likelihood for a reliability experiment with right-censored data is

$$
L\left(\gamma, \beta, \sigma_{\omega} \mid \text { Data }\right)=\int \prod_{i j}\left[f_{1}\left(t_{j(i)} \mid \omega_{i}\right)\right]^{\delta_{j(i)}}\left[1-F_{1}\left(t_{j(i)} \mid \omega_{i}\right)\right]^{1-\delta_{j(i)}} f_{2}\left(\omega_{i}\right) d \omega_{i}
$$

where $\delta_{j(i)}=1$ if the observation represents a failure and 0 otherwise. The challenge lies in integrating out the random effect from the joint likelihood. Freeman and Vining (2012) proposes a method implementing Gauss-Hermite quadrature to integrate out the random effect leading to a closed form approximation to the joint log-likelihood. McCulloch and Searle (2001) explains the implementation of Gauss-Hermite quadrature. A rearranged form of this joint likelihood is 


$$
L\left(\boldsymbol{\gamma}, \beta, \sigma_{\omega} \mid \text { Data }\right)=\prod_{i=1}^{m}\left[\int_{-\infty}^{\infty} \prod_{j=1}^{n_{i}}\left[f_{1}\left(t_{j(i)} \mid \omega_{i}\right)\right]^{\delta_{j(i)}}\left[1-F_{1}\left(t_{j(i)} \mid \omega_{i}\right)\right]^{1-\delta_{j(i)}} f_{2}\left(\omega_{i}\right)\right] d \omega_{i} .
$$

Next, substitute the formulas $f_{1}\left(t_{j(i)} \mid \omega_{i}\right), F_{1}\left(t_{j(i)} \mid \omega_{i}\right)$ and $f_{2}\left(\omega_{i}\right)$ into the above equation to obtain

$$
L\left(\boldsymbol{\gamma}, \beta, \sigma_{\omega} \mid \text { Data }\right)=\prod_{i=1}^{m}\left[\int_{-\infty}^{\infty} \prod_{j=1}^{n_{i}} g\left(t_{j(i)} \mid \omega_{i}\right) \times \frac{1}{\sqrt{2 \pi \sigma_{\omega}^{2}}} e^{-\frac{\omega_{i}^{2}}{2 \sigma_{\omega}^{2}}}\right] d \omega_{i},
$$

where $g\left(t_{j(i)} \mid \omega_{i}\right)=\left[f_{1}\left(t_{j(i)} \mid \omega_{i}\right)\right]^{\delta_{j(i)}}\left[1-F_{1}\left(t_{j(i)} \mid \omega_{i}\right)\right]^{1-\delta_{j(i)}}$. Using the substitution $\omega_{i}=\sqrt{2} \sigma_{\omega} v_{i}$ gives

$$
L\left(\boldsymbol{\gamma}, \beta, \sigma_{\omega} \mid \text { Data }\right)=\prod_{i=1}^{m}\left[\int_{-\infty}^{\infty} \prod_{j=1}^{n_{i}} g\left(t_{j(i)} \mid \sqrt{2} \sigma_{\omega} v_{i}\right) \times \frac{1}{\sqrt{\pi}} e^{-v_{i}^{2}}\right] d v_{i} .
$$

Applying Gauss-Hermite quadrature results in a closed form approximation to the likelihood

$$
L\left(\boldsymbol{\gamma}, \beta, \sigma_{\omega} \mid \text { Data }\right) \approx \prod_{i=1}^{m} \frac{1}{\sqrt{\pi}}\left[\sum_{k=1}^{n_{k}}\left[\prod_{j=1}^{n_{i}} g\left(t_{j(i)} \mid \sqrt{2} \sigma_{\omega} q_{k}\right) w_{k}\right]\right]
$$

where $n_{k}$ is the number of quadrature points, $w_{k}$ are the weights and $q_{k}$ are the evaluation points. The weights are 


$$
w_{k}=\frac{2^{n-1} n ! \sqrt{\pi}}{n^{2}\left[H_{n-1}\left(q_{k}\right)\right]^{2}},
$$

where $H_{n}(q)$ is the Hermite polynomial of degree $n$. The evaluation points, $q_{k}$, are the roots of the Hermite polynomial. Abramowitz and Stegun (1964) supplies tables of the weights and evaluation points for several values of $n$. An approximate log-likelihood is

$$
\mathcal{L}\left(\boldsymbol{\gamma}, \beta, \sigma_{\omega} \mid \text { Data }\right) \approx \sum_{i=1}^{m} \log \left(\frac{1}{\sqrt{\pi}} \sum_{k=1}^{n_{k}}\left[\prod_{j=1}^{n_{i}} g\left(t_{j(i)} \mid \sqrt{2} \sigma_{\omega} q_{k}\right) w_{k}\right]\right) .
$$

This approximation to the log-likelihood allows the derivation of an asymptotic variancecovariance matrix allowing one to make inferences on functions of the parameters. 


\section{Chapter 3}

\section{Reliability Experiments with}

\section{Subsampling}

This chapter provides a preliminary exploration of the two-stage and NLMM analyses for reliability experiments with subsampling introduced in Chapter 2. The performance of these methods is evaluated in a simulation study that provides the framework for later simulation studies involving reliability experiments with random blocks and subsampling. The simulation study in Freeman and Vining (2012) examines the estimation of parameters under model misspecification; whereas this simulation study focuses on the type I error rate and power for testing factor effects. 


\subsection{Simulation Study}

The performance of the NLMM method, two-stage method and traditional analysis (which assumes a completely randomized design) is compared via simulations in SAS using 1,000 replicates. As discussed in Chapter 2 the NLMM method uses an approximate likelihood, which is computed from 20 quadrature points in these simulations. This study evaluates the bias and variability of model parameters as well as the type I error rate and power.

The simulation scenario involves failure time data with subsampling. In this scenario, manufacturers seek to determine the effect of two operating conditions on product life. Operating condition is denoted by $x$ (with two levels coded -1 and 1 ). Each treatment is replicated 4 times, giving 8 experimental units. The experimental setup is such that each experimental unit contains $n$ items (observational units). The simulation records the first $r$ failures for each experimental unit, employing type II censoring. Simulations are completed for scenarios where failure times follow a Weibull distribution with shape parameter $\beta=5,3,1$, and 0.5 , and $\log$-scale parameter $\log \left(\eta_{i}\right)=\mu_{i}=\gamma_{0}+\gamma_{1} x_{i}+\omega_{i}$ with $\omega_{i} \sim N\left(0, \sigma_{\omega}^{2}\right)$. This simulation study explores three combinations of $n$ and $r: n=8$ and $r=4, n=16$ and $r=8$, and $n=16$ and $r=12$. Simulations are completed for $\sigma_{\omega}=0.1$ and 0.3162 . The parameter $\gamma_{0}$ remains fixed at 5 , while $\gamma_{1}$ changes based on the value of $\sigma_{\omega}$ and the nominal power. 
Figure 3.1 shows the ratio of $\hat{\beta}$ to $\beta$.

Figure 3.1: Ratio of $\hat{\beta}$ to $\beta$

(a) $\sigma_{\omega}=0.1$

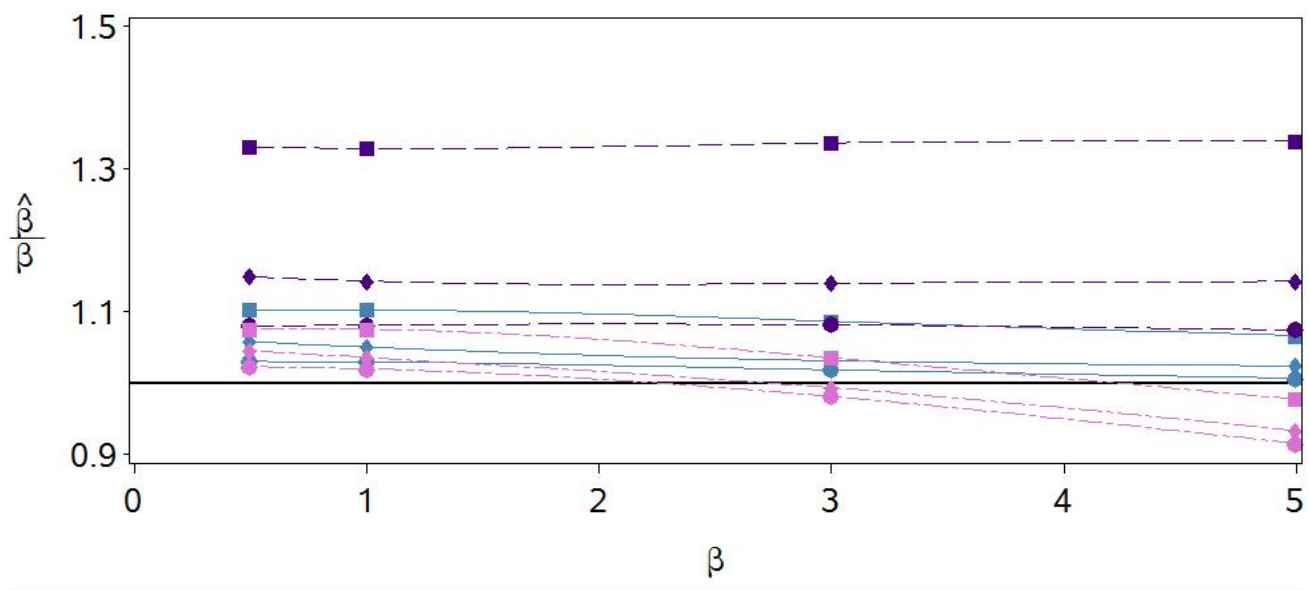

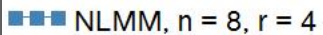
-E Two-Stage, $n=8, r=4$ 메를 Traditional, $\mathrm{n}=8, \mathrm{r}=4$

NLMM, $n=16, r=8$ $\cdots$ Two-Stage, $n=16, r=8$

NLMM, $n=16, r=12$

Two-Stage, $n=16, r=12$

Traditional, $n=16, r=12$

(b) $\sigma_{\omega}=0.3162$

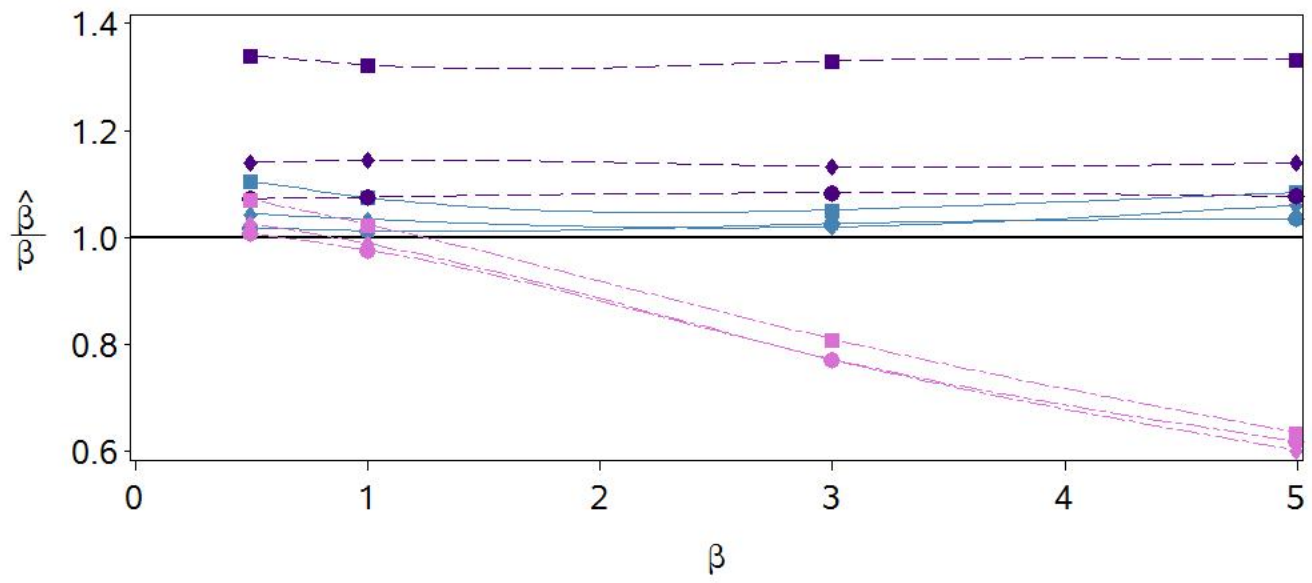

NLMM, $\mathrm{n}=8, \mathrm{r}=4$

Two-Stage, $n=8, r=4$

win Traditional, $n=8, r=4$
NLMM, $\mathrm{n}=16, \mathrm{r}=8$

$\bullet$ Two-Stage, $n=16, r=8$

Traditional, $n=16, r=8$
NLMM, $n=16, r=12$

- Two-Stage, $n=16, r=12$ Traditional, $n=16, r=12$ 
The NLMM method has the least bias in estimating $\beta$, and performs increasingly better as the number of failures per test stand increases. The two-stage method produces biased estimates of $\beta$; however, the bias is invariant to $\beta$ and decreases as the sample size increases. The bias' invariance to $\beta$ may make it possible to find a bias correction factor, as discussed in Chapter 2. The bias of the traditional method depends on $\beta$ and becomes more severe as $\beta$ increases. Furthermore, as $\sigma_{\omega}$ increases the traditional method's estimation of $\beta$ deteriorates. 
Figure 3.2 depicts the estimates of $\gamma_{0}=5$.

Figure 3.2: $\hat{\gamma}_{0}$

(a) $\gamma_{0}=5, \sigma_{\omega}=0.1$

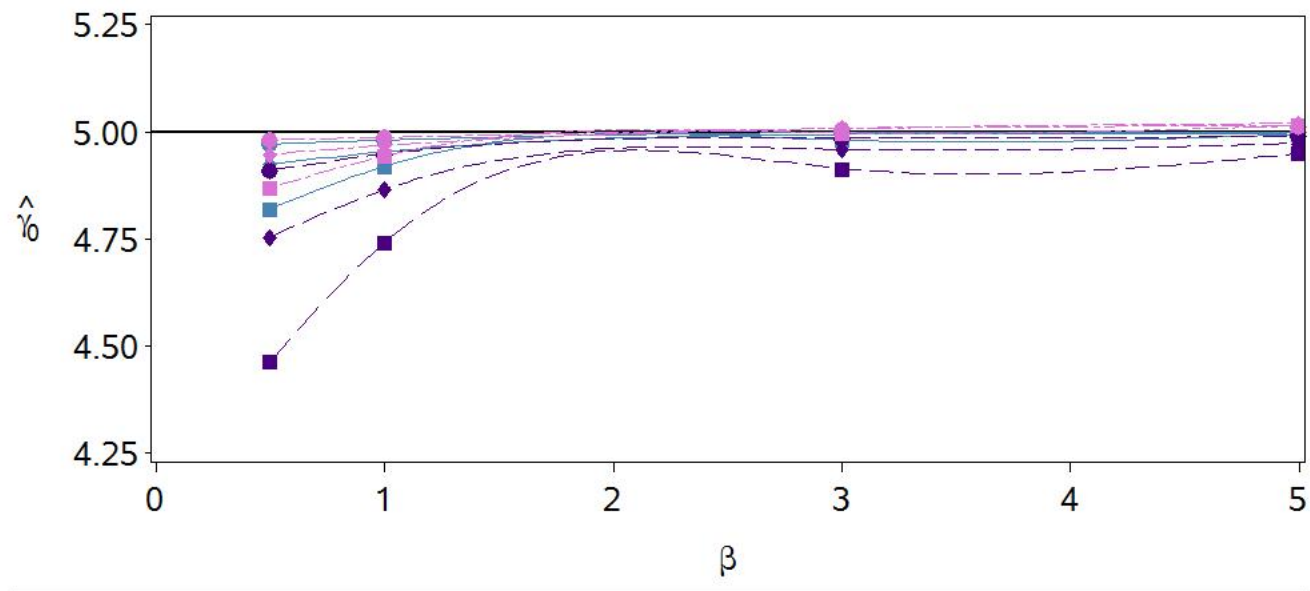

마므. $\mathrm{NLMM}=8, \mathrm{r}=4$

Two-Stage, $n=8, r=4$

NLMM, $n=16, r=8$

$\bullet$ Two-Stage, $n=16, r=8$

NLMM, $n=16, r=12$

ㅁin Traditional, $n=8, r=4$

$\leftrightarrow$ Traditional, $n=16, r=8$

Two-Stage, $n=16, r=12$

Traditional, $n=16, r=12$

(b) $\gamma_{0}=5, \sigma_{\omega}=0.3162$

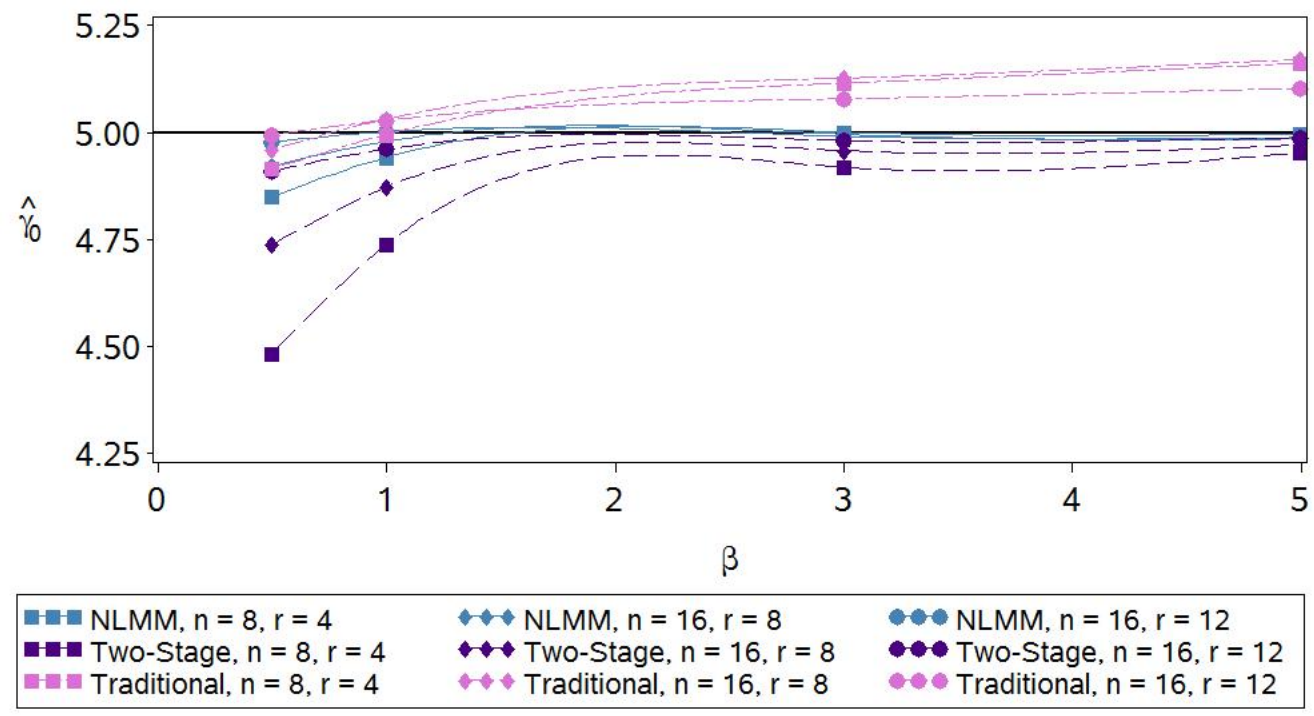


The NLMM and two-stage methods perform consistently for both values of $\sigma_{\omega}$; whereas, the estimate of $\gamma_{0}$ from the traditional method has a larger bias for the larger value of $\sigma_{\omega}$. Overall, the NLMM performs the best in the estimation of $\gamma_{0}$, and for all three methods the estimation improves as the number of failures on a test stand increases. 
Figure 3.3 gives the estimates of $\gamma_{1}=0$, which is the value of $\gamma_{1}$ under the null hypothesis that $x$ does not affect the time to failure.

Figure 3.3: $\hat{\gamma}_{1}$

(a) $\gamma_{1}=0, \sigma_{\omega}=0.1$

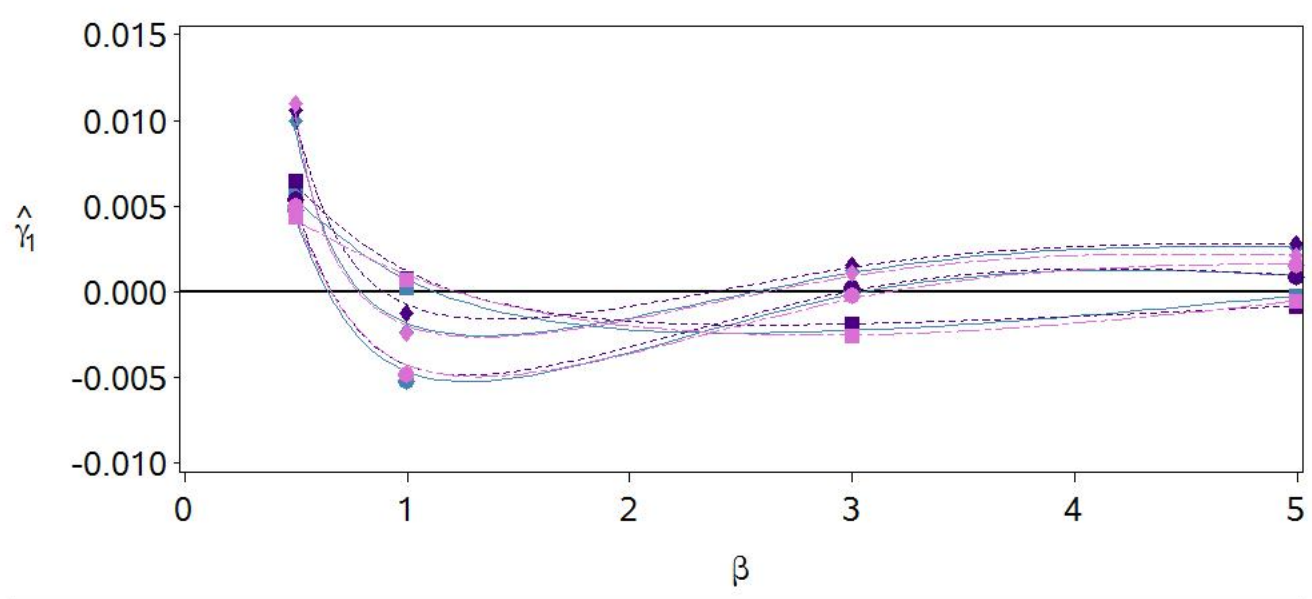

(b) $\gamma_{1}=0, \sigma_{\omega}=0.3162$

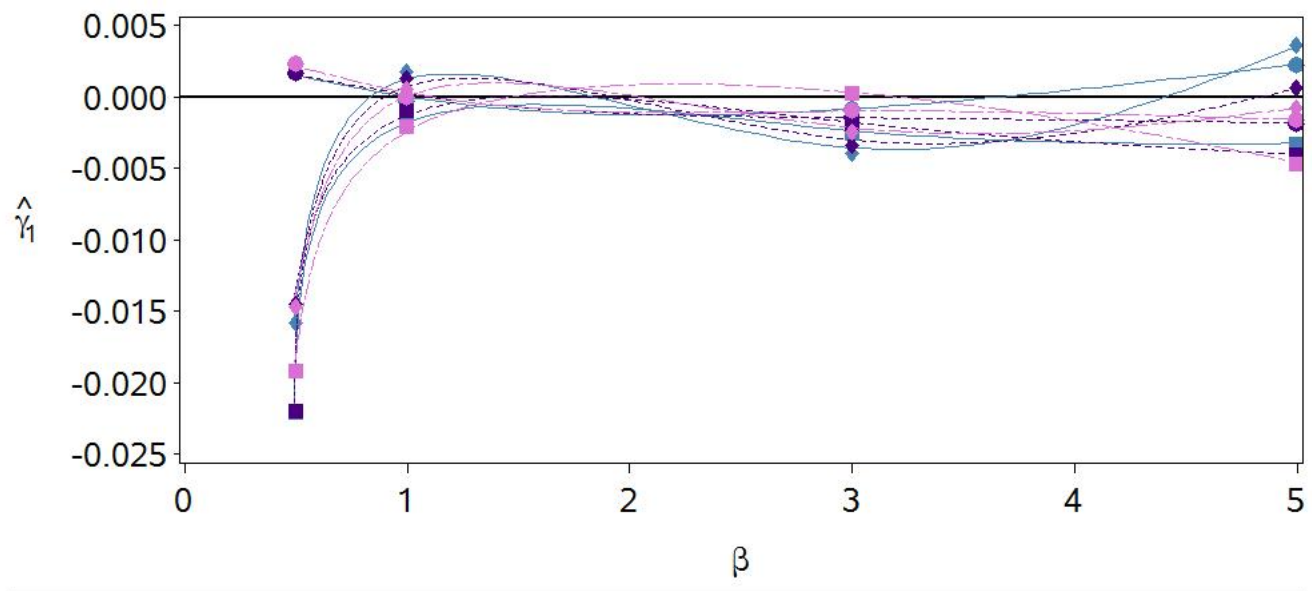

NLMM, $\mathrm{n}=8, \mathrm{r}=4$

Two-Stage, $n=8, r=4$

마를 Traditional, $n=8, r=4$
NLMM, $n=16, r=8$ $\bullet$ Two-Stage, $n=16, r=8$ $\leftrightarrow$ Traditional, $n=16, r=8$
NLMM, $n=16, r=12$

Two-Stage, $n=16, r=12$ Traditional, $n=16, r=12$ 
All three methods perform similarly, with sample size affecting the performance of the estimation. Figure 3.4 shows estimates of $\gamma_{1}$, where the true value of $\gamma_{1}$ is calculated to have a nominal power of 0.50 under stage 2 in the two-stage method. Under the assumption that $\gamma_{1}=0, P\left(F^{*}>F_{0.95,1,6, \lambda=0}=5.987378\right)=0.05$ where $F^{*}$ is the observed $F$ statistic in stage 2 of the two-stage analysis. For the experimental design in this simulation scenario, the noncentrality parameter is $\lambda=\frac{8 \gamma_{1}^{2}}{\sigma_{\omega}^{2}}$ (see Graybill (1976) for deriving the noncentrality parameter). To find the value of $\gamma_{1}$ that will give a nominal power of 0.50 , first find the value of $\lambda$ such that $P\left(F^{*}>5.987378 \mid \lambda\right)=0.50$. The value of $\lambda$ for which this equation holds is 5.454222. Next, substitute this value of $\lambda$ into $\lambda=\frac{8 \gamma_{1}^{2}}{\sigma_{\omega}^{2}}$. Solving for $\gamma_{1}$ gives $\gamma_{1}= \pm 0.08257$ and $\gamma_{1}= \pm 0.26111$ for $\sigma_{\omega}=0.1$ and $\sigma_{\omega}=0.3162$ respectively. This simulation study uses the positive solutions of $\gamma_{1}$. 
Figure 3.4 illustrates the estimates of $\gamma_{1}$ when calibrated for a nominal power of 0.50 .

Figure 3.4: $\hat{\gamma}_{1}$ for Nominal Power 0.50

(a) $\gamma_{1}=0.08257$ and $\sigma_{\omega}=0.1$

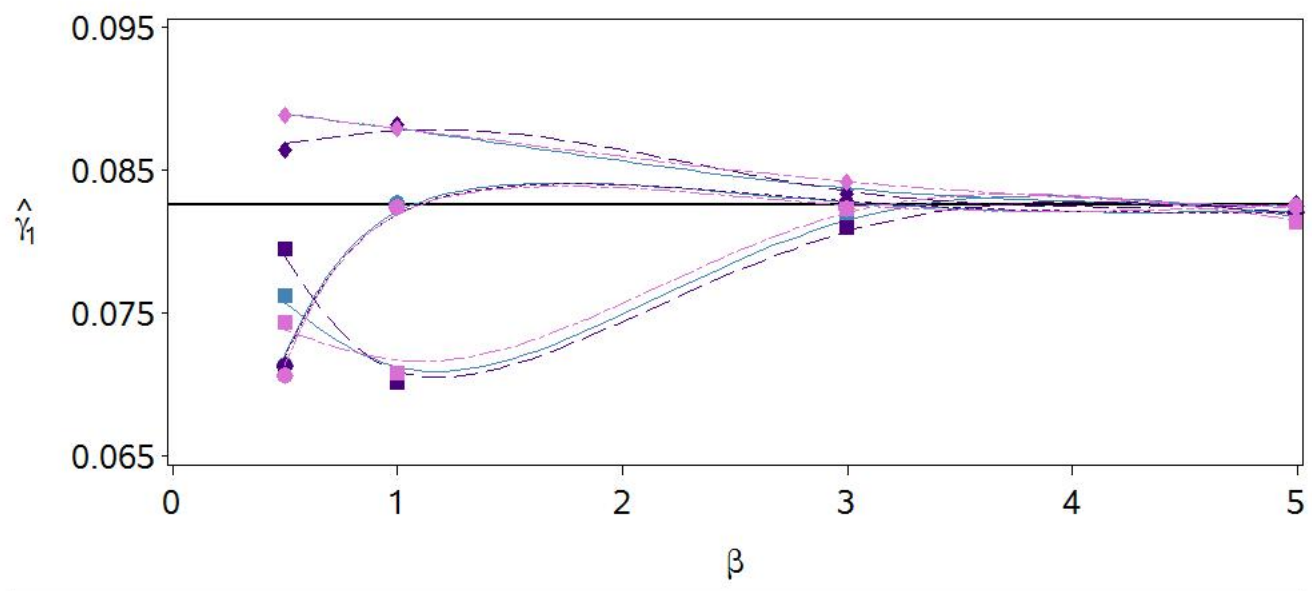

NLMM, $\mathrm{n}=8, \mathrm{r}=4 \mathrm{NLMM}, \mathrm{n}=16, \mathrm{r}=8$

Two-Stage, $n=8, r=4$

말 $\leftrightarrow$ Two-Stage, $n=16, r=8$

Traditional, $n=16, r=8$
NLMM, $n=16, r=12$

Two-Stage, $n=16, r=12$

Traditional, $n=16, r=12$

(b) $\gamma_{1}=0.26111$ and $\sigma_{\omega}=0.3162$

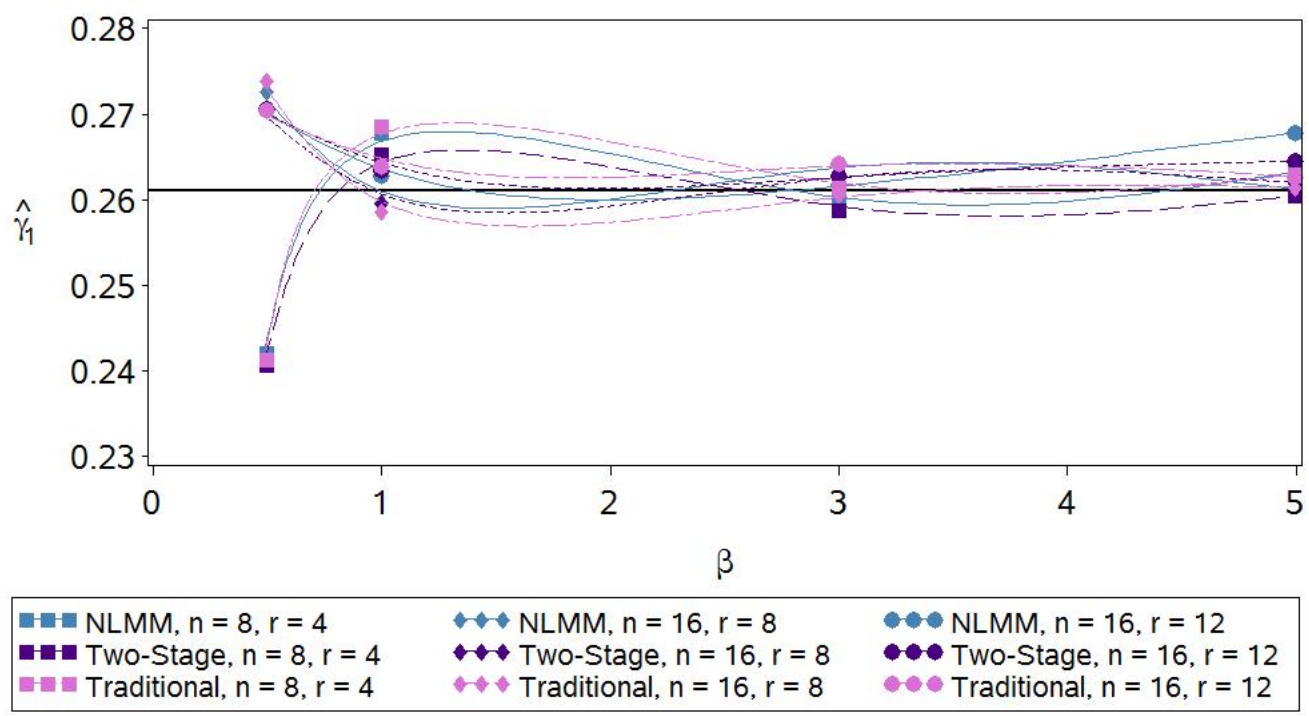


Estimation of $\gamma_{1}$ is very similar for the NLMM, two-stage and traditional analyses. Figure 3.5 juxtaposes the estimates of $\gamma_{1}$ for a nominal power of 0.80 .

Figure 3.5: $\hat{\gamma}_{1}$ for Nominal Power 0.80

(a) $\gamma_{1}=0.11904$ for $\sigma_{\omega}=0.1$

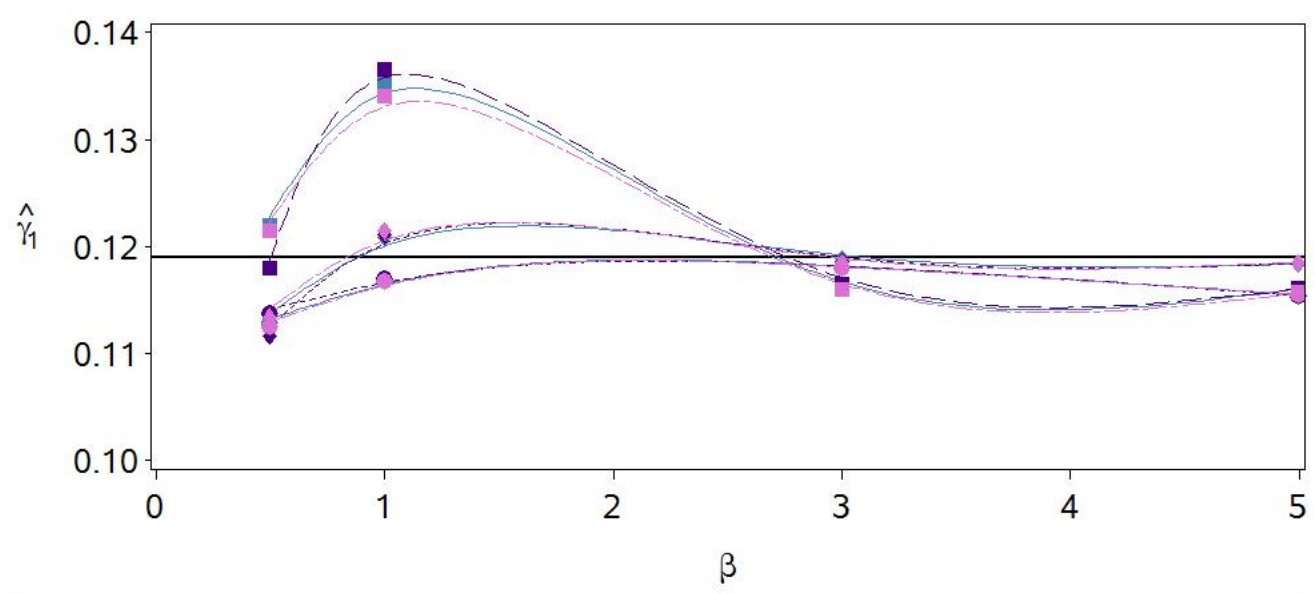

(b) $\gamma_{1}=0.37643$ for $\sigma_{\omega}=0.3162$

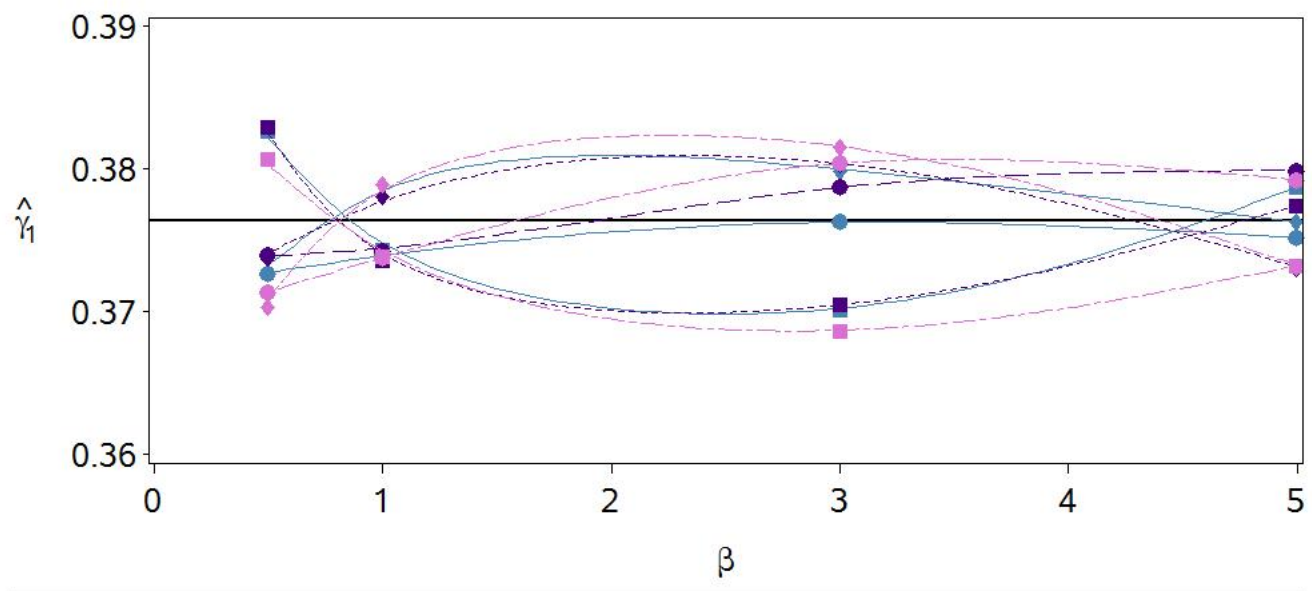

NLMM, $\mathrm{n}=8, \mathrm{r}=4$ - Two-Stage, $n=8, r=4$ 마를 Traditional, $n=8, r=4$
NLMM, $\mathrm{n}=16, \mathrm{r}=8$ $\bullet$ Two-Stage, $n=16, r=8$ $\leftrightarrow$ Traditional, $n=16, r=8$
NLMM, $n=16, r=12$

Two-Stage, $n=16, r=12$ Traditional, $n=16, r=12$ 
Again, estimates of $\gamma_{1}$ appear similar for all three methods. Figure 3.6 shows the estimates of $\gamma_{1}$ for a nominal power of 0.90 .

Figure 3.6: $\hat{\gamma}_{1}$ for Nominal Power 0.90

(a) $\gamma_{1}=0.13833$ for $\sigma_{\omega}=0.1$

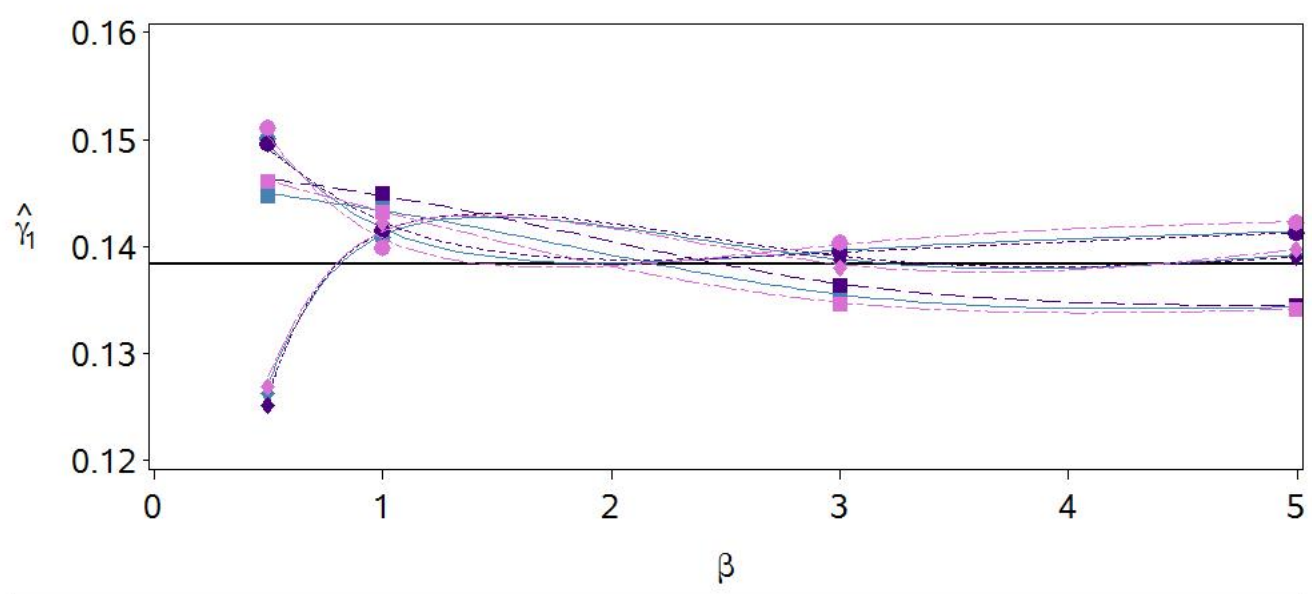

(b) $\gamma_{1}=0.43744$ for $\sigma_{\omega}=0.3162$

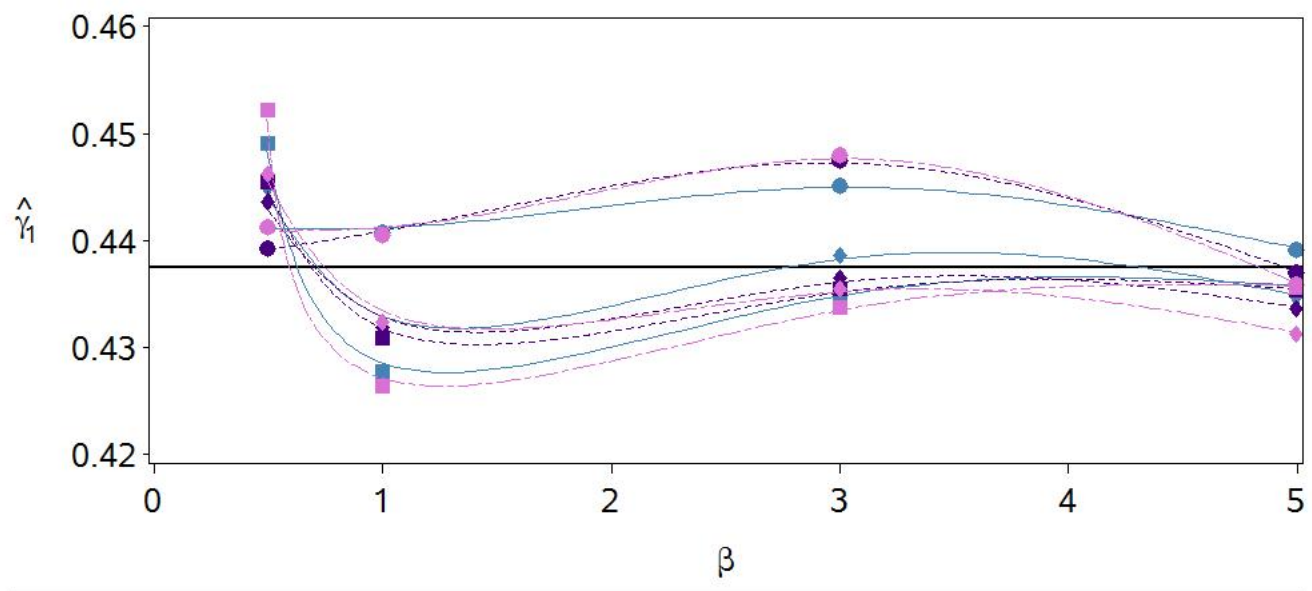

NLMM, $\mathrm{n}=8, \mathrm{r}=4$

- Two-Stage, $n=8, r=4$

말
NLMM, $\mathrm{n}=16, \mathrm{r}=8$

$\bullet$ Two-Stage, $n=16, r=8$

$\leftrightarrow$ Traditional, $n=16, r=8$
NLMM, $n=16, r=12$

Two-Stage, $n=16, r=12$

Traditional, $n=16, r=12$ 
Figures 3.3 through 3.6 show that the NLMM, two-stage and traditional analyses produce similar estimates for $\gamma_{1}$. The bias of the estimates depends on the number of failures per test stand, $\beta$ and $\sigma_{\omega}$. Figure 3.7 compares the performance of the estimation of $\sigma_{\omega}$ for the NLMM and two-stage methods (the traditional method does not provide an estimate for $\left.\sigma_{\omega}\right)$ 
Figure 3.7: $\hat{\sigma}_{\omega}$

(a) $\sigma_{\omega}=0.1$

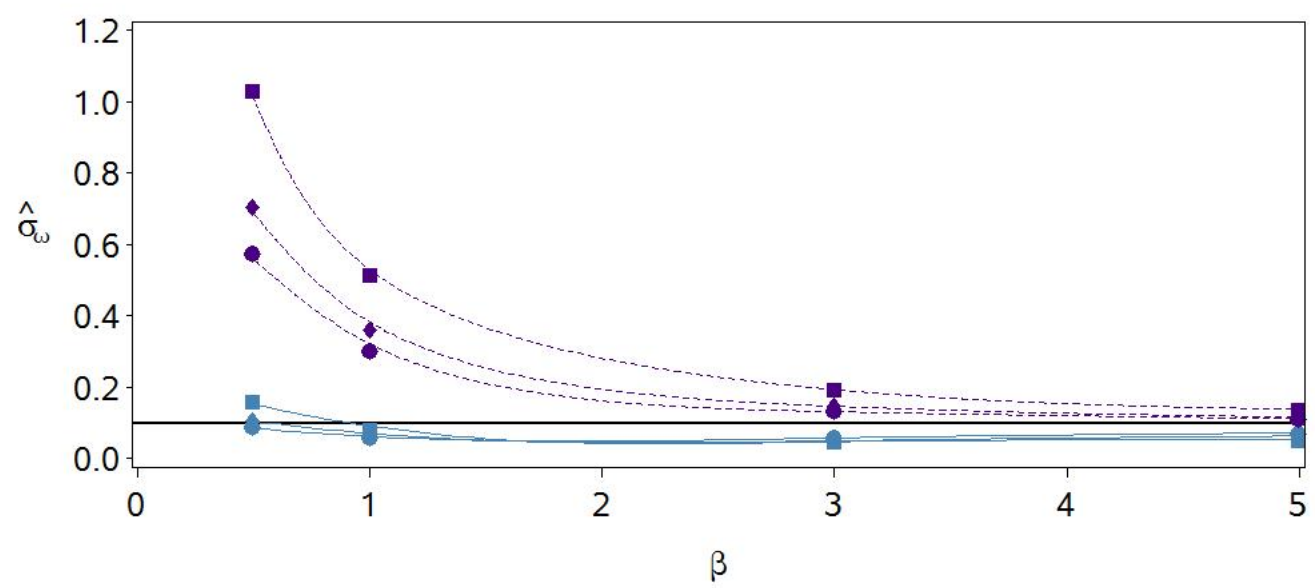

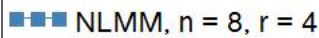

Two-Stage, $n=8, r=4$

NLMM, $n=16, r=8$

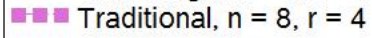

$\bullet$ Two-Stage, $n=16, r=8$

NLMM, $n=16, r=12$

Traditional, $n=16, r=8$

- Two-Stage, $n=16, r=12$

Traditional, $n=16, r=12$

(b) $\sigma_{\omega}=0.3162$

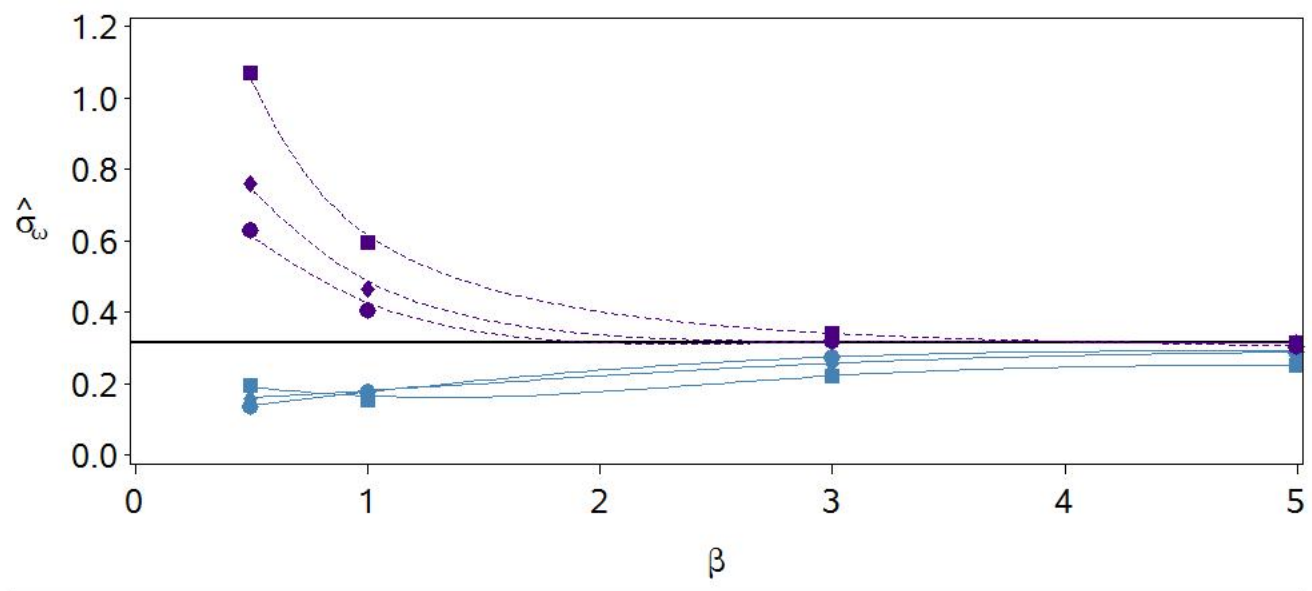

NLMM, $\mathrm{n}=8, \mathrm{r}=4$

Two-Stage, $n=8, r=4$

$\cdots$ NLMM, $n=16, r=8$

NLMM, $n=16, r=12$

물 Traditional, $n=8, r=4$

$\cdots$ Two-Stage, $n=16, r=8$

Two-Stage, $n=16, r=12$

$\leftrightarrow$ Traditional, $n=16, r=8$

Traditional, $n=16, r=12$ 
Figure 3.7 shows that the NLMM provides a much better estimate of $\sigma_{\omega}$ than the two-stage method. Figure 3.8 depicts the empirical error for a test on $\gamma_{1}$ under a nominal type I error rate of 0.05. For the NLMM simulations examine the power of two different testing methods: the first method is the likelihood ratio test and the second is the Wald test. 
Figure 3.8: Empirical Error Rate

(a) $\sigma_{\omega}=0.1$

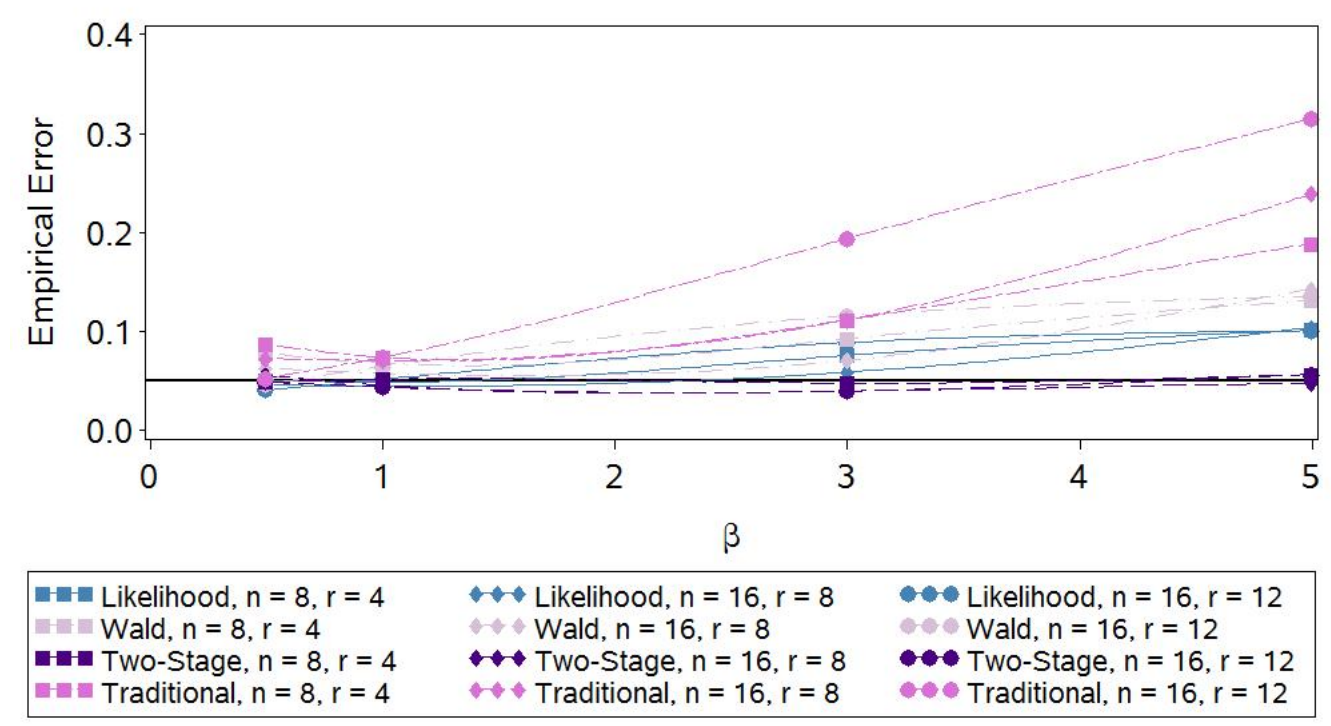

(b) $\sigma_{\omega}=0.3162$

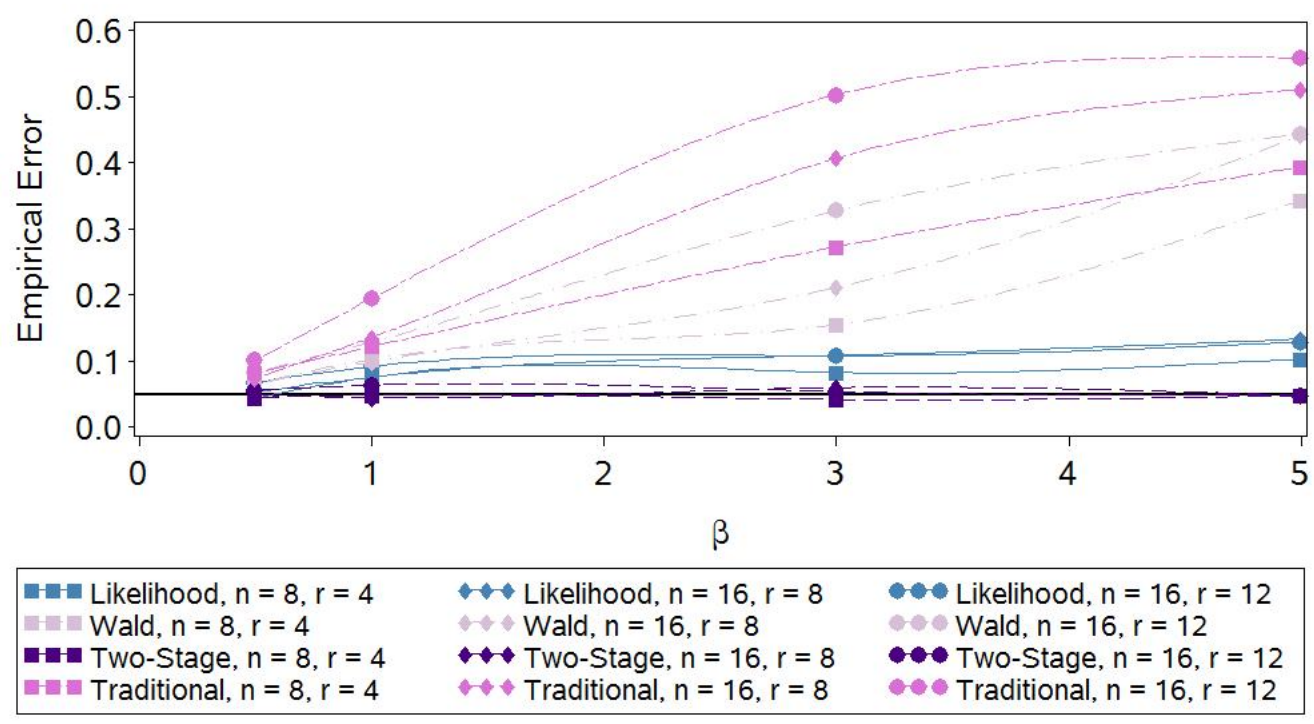


Figure 3.8 indicates that the two-stage method maintains the nominal type I error rate. The likelihood ratio test for the NLMM has an empirical error that for large values of $\beta$ increases to 0.10 or 0.15 , depending on the test stand error. The Wald and traditional methods have very high empirical errors, particularly for $\beta=3$ and $\beta=5$. Figure 3.9 illustrates the empirical power of a test on $\gamma_{1}$ under a nominal power of 0.50 and a type I error rate of 0.05 . 
Figure 3.9: Nominal Power of 0.50

(a) $\sigma_{\omega}=0.1$

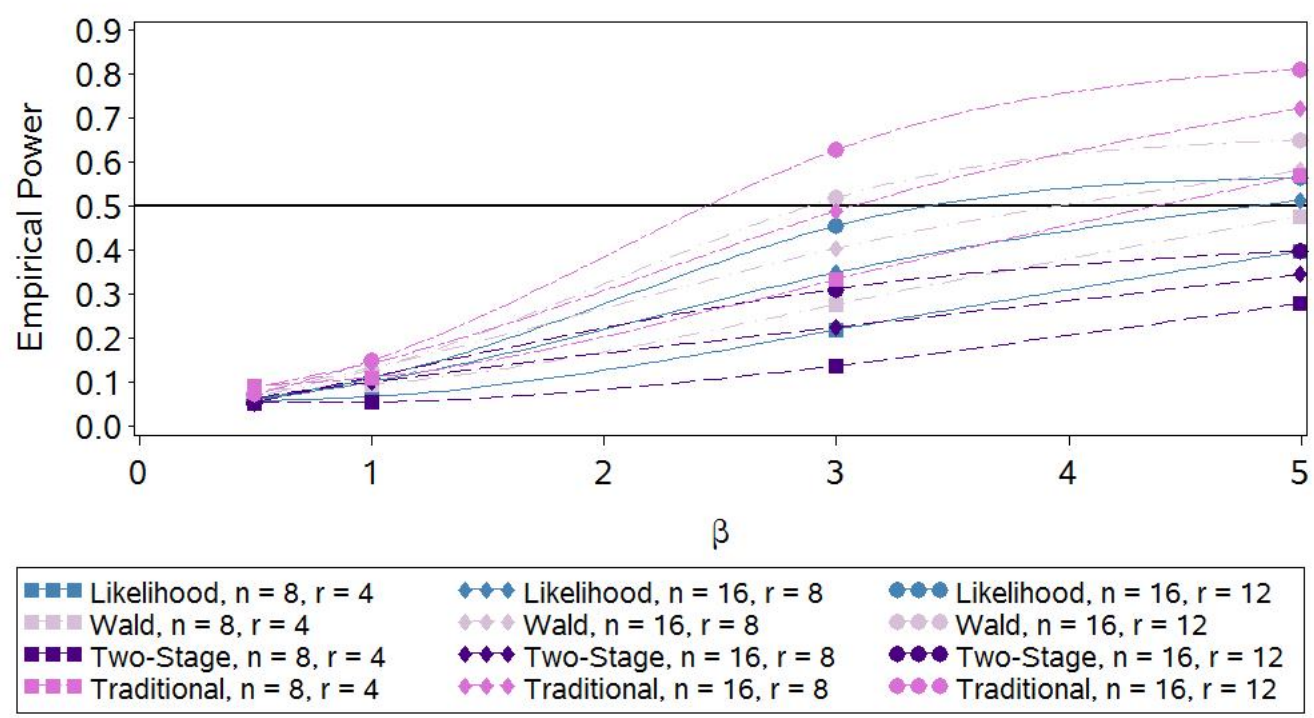

(b) $\sigma_{\omega}=0.3162$

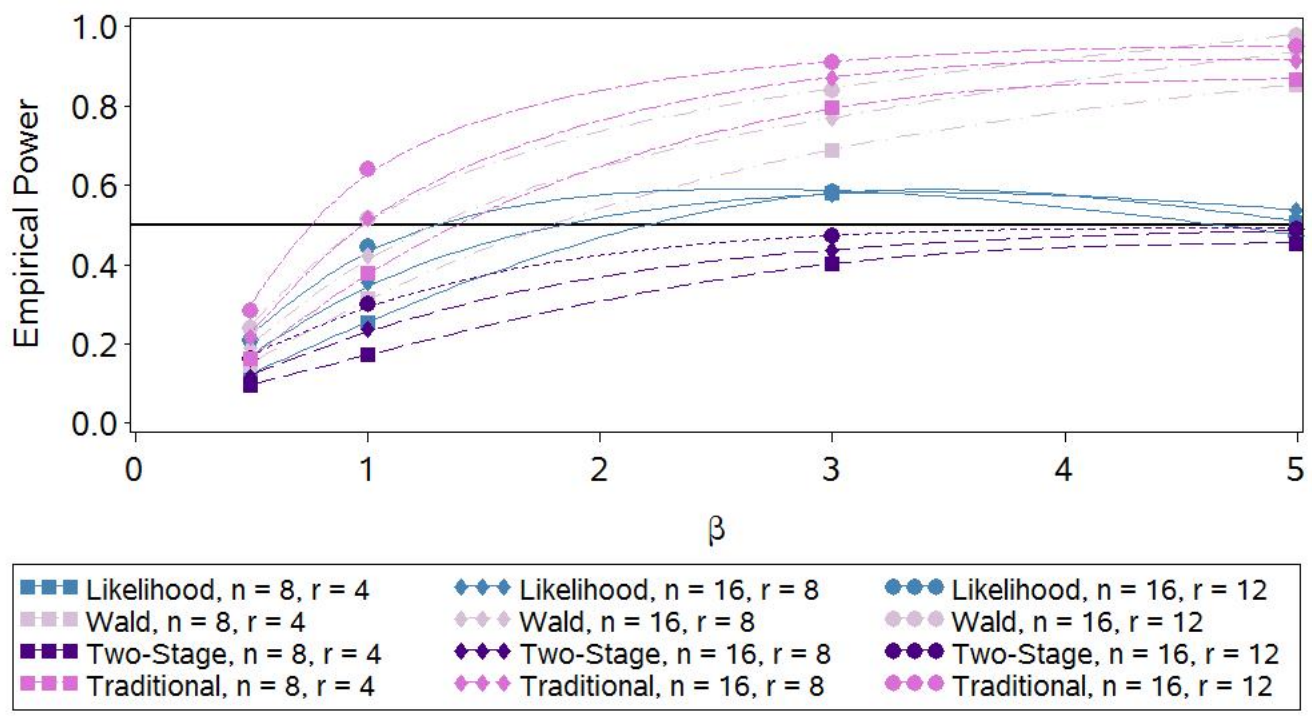


Figure 3.10 presents the empirical power of the three methods for a nominal power of 0.80 and a nominal type I error rate of 0.05 .

Figure 3.10: Nominal Power of 0.80

$$
\text { (a) } \sigma_{\omega}=0.1
$$

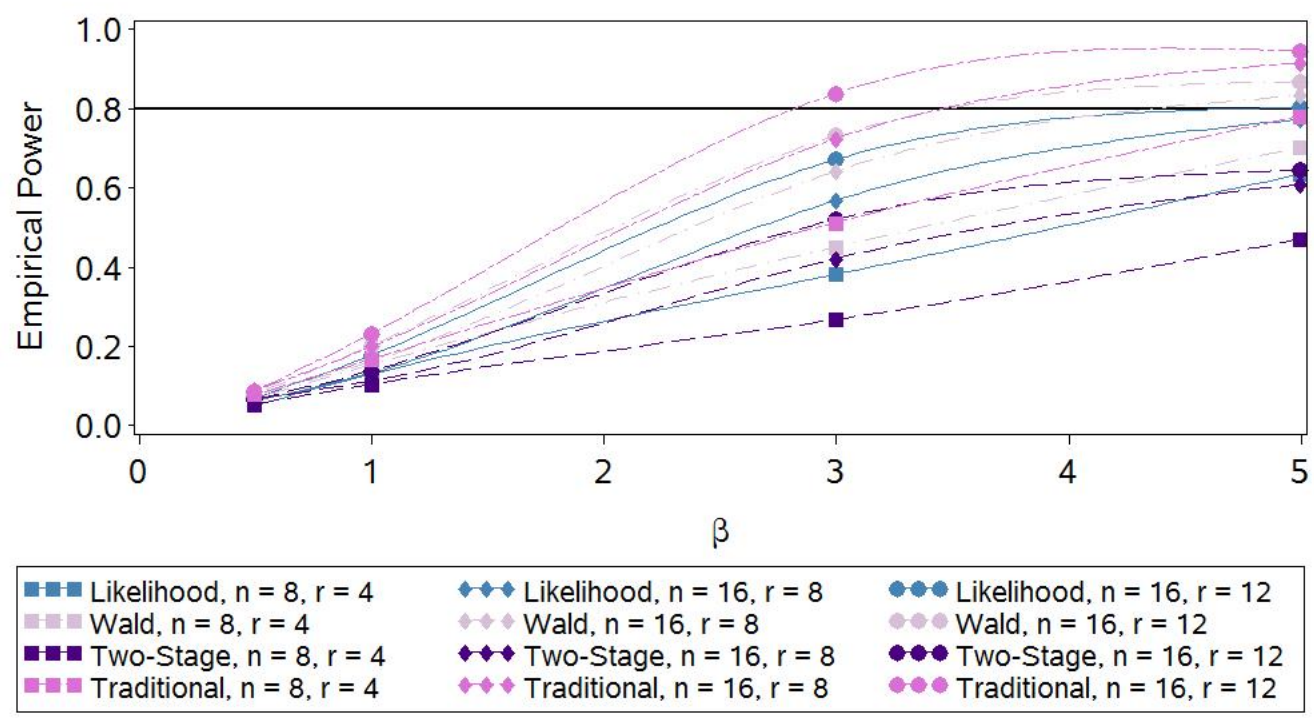

(b) $\sigma_{\omega}=0.3162$

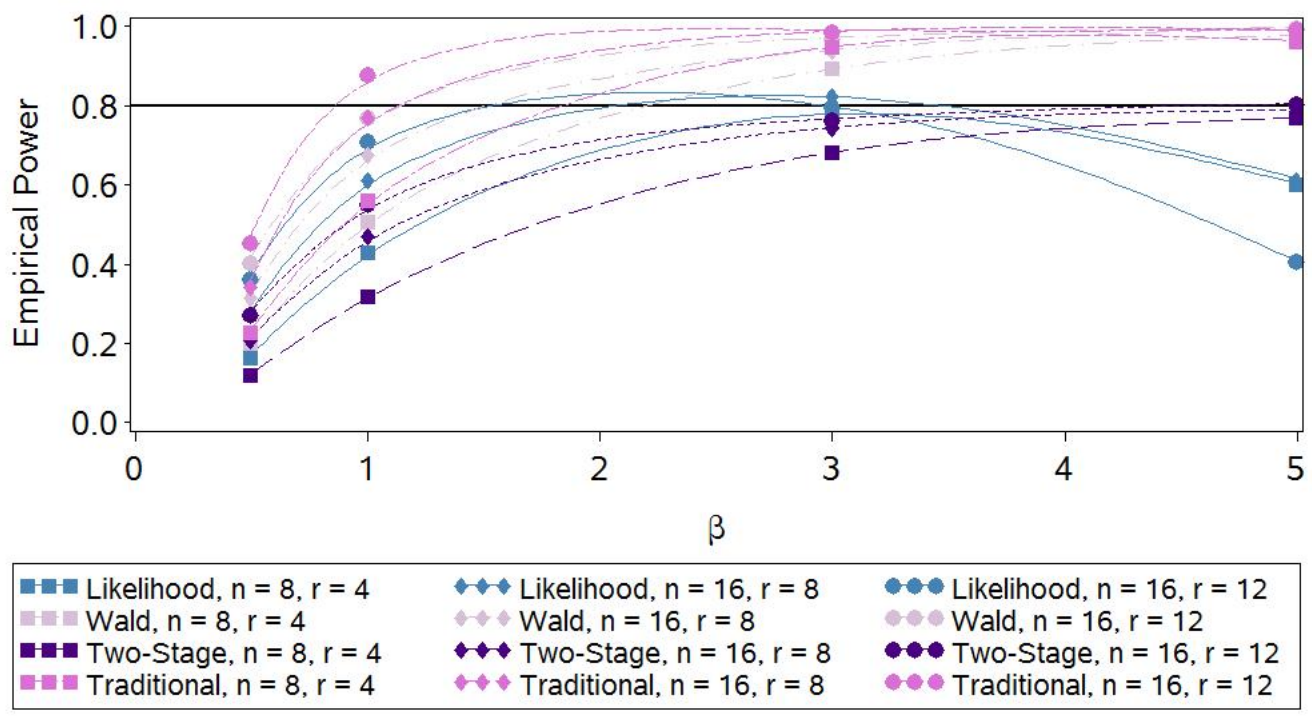


Figure 3.11 displays the empirical power under a nominal power of 0.90 .

Figure 3.11: Nominal Power of 0.90

(a) $\sigma_{\omega}=0.1$

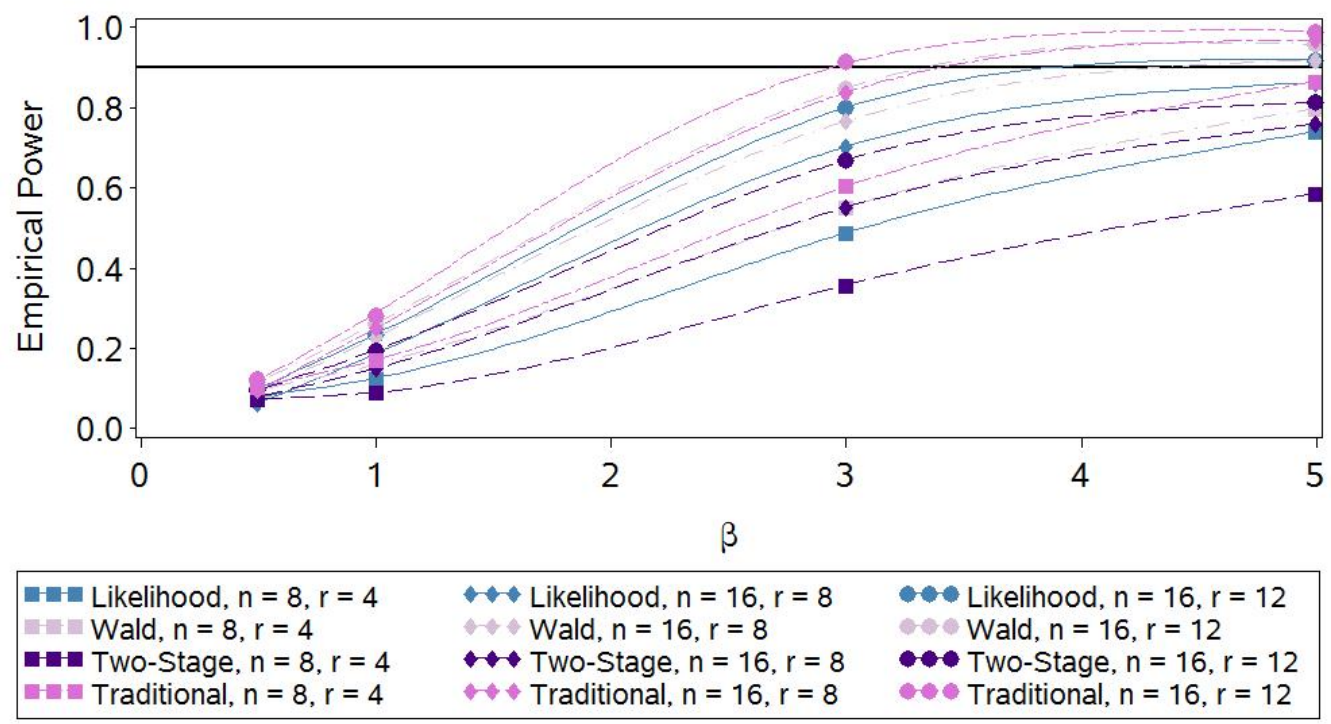

(b) $\sigma_{\omega}=0.3162$

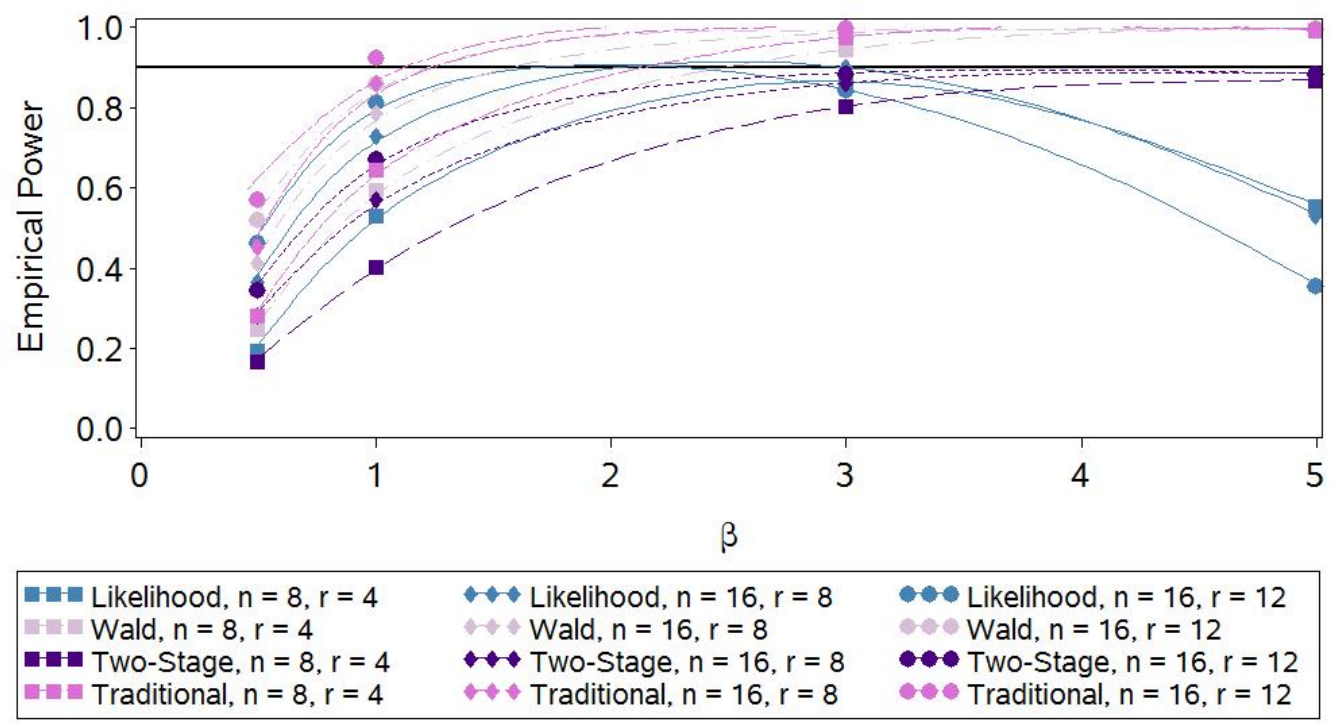




\subsection{Conclusions}

In terms of parameter estimation, the performance of the NLMM and two-stage methods does not depend on whether $\sigma_{\omega}=0.1$ or $\sigma_{\omega}=0.3162$. On the other hand, the completely randomized traditional analysis performs worse for $\sigma_{\omega}=0.3162$ than for $\sigma_{\omega}=0.1$. This implies that the impact of failing to incorporate the test stand error into the model increases as the test stand error increases.

In terms of testing, the two-stage method preserves the nominal type I error rate for all values of $\beta$ and for the different values of $\sigma_{\omega}$. Caution should be used in comparing the empirical powers to the nominal powers. The nominal power values are calculated based on stage 2 of the two-stage model, which does not depend on $\beta$. However, because $\beta$ impacts the variance of the Weibull distribution, it affects the power. Unfortunately, theory for a closed form power calculation does not exist. Note that as $\beta$ increases and as the number of failures per test stand increases, the empirical power using the two-stage method approaches the nominal power. For the likelihood ratio test the empirical power appears closest to the nominal power for $\beta=3$. When $\beta=3$ the Weibull distribution closely resembles the normal distribution. One possibility is that convergence to the asymptotic distribution has not yet occurred for other values of $\beta$.

This chapter uses simulations to compare the methods Freeman and Vining (2010, 2012) propose with the traditional analysis assuming a completely randomized design. These simulations provide insight into how the various methods deal with different magnitudes of 
the test stand error. The insight gained from these simulations will be essential in designing simulations with both a test stand error and a random block error. 


\section{Chapter 4}

\section{Two-Stage Analysis}

This chapter provides a straightforward, easy to implement modeling approach that handles reliability data containing random blocks with subsampling. Although random blocks are common in many types of experiments, current methods do not properly incorporate this type of experimental protocol into the analysis for the Weibull distribution. This chapter provides a technique that is an improvement over the current method, which assumes the independence of all units (i.e. a completely randomized design). In addition, commercial statistical software is used to conduct the analysis making it easy to implement.

\subsection{Random Blocks with Subsampling}

Consider a reliability experiment in which the experimenter places $n$ items on a test stand and records the failure or censoring time. The manufacturer produces the items in batches, 
but a single batch cannot produce enough items for the entire experiment. In this case, the batches represent random blocks. Taking into account these random blocks in the analysis represents a form of local control of error.

Furthermore, suppose the researcher has $l$ batches of items available for the experiment. Each batch feeds $m$ test stands for a total of $l m$ test stands. The engineer applies a treatment combination to each test stand and places $n$ items (from the same batch) on each test stand. In this scenario the experiment contains subsampling, where the test stand represents the experimental unit and the item represents the observational unit. Let $t_{k(i j)}$ be the failure time for the $k^{\text {th }}$ observational unit within the $j^{\text {th }}$ experimental unit for the $i^{\text {th }}$ block. Figure 4.1 gives a graphical display of the experimental setup.

Figure 4.1: Experimental Setup

\begin{tabular}{|c|c|c|c|c|c|}
\hline \multicolumn{2}{|c|}{ Batch 1} & \multicolumn{2}{|c|}{ Batch 2} & \multicolumn{2}{|c|}{ Batch $l$} \\
\hline & $\searrow \mathrm{m}$ & & 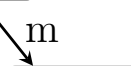 & & \\
\hline $\begin{array}{l}\text { Test } \\
\text { Stand }\end{array}$ & $\begin{array}{l}\text { Test } \\
\text { Stand }\end{array}$ & $\begin{array}{l}\text { Test } \\
\text { Stand }\end{array}$ & $\begin{array}{l}\text { Test } \\
\text { Stand }\end{array}$ & Test & $\begin{array}{l}\text { Test } \\
\text { Stan }\end{array}$ \\
\hline$\downarrow$ & $\downarrow$ & $\downarrow$ & $\downarrow$ & $\downarrow$ & $\downarrow$ \\
\hline $\mathrm{n}$ & $\begin{array}{c}n \\
\text { ittems }\end{array}$ & $\begin{array}{c}n \\
\text { items }\end{array}$ & $\begin{array}{c}n \\
\text { itcm }\end{array}$ & $\begin{array}{c}n \\
\text { itcme }\end{array}$ & $\begin{array}{c}\mathrm{n} \\
\mathrm{itom}\end{array}$ \\
\hline items & items & items & 1tems & items & \\
\hline
\end{tabular}

If the failure times follow the Weibull distribution, then

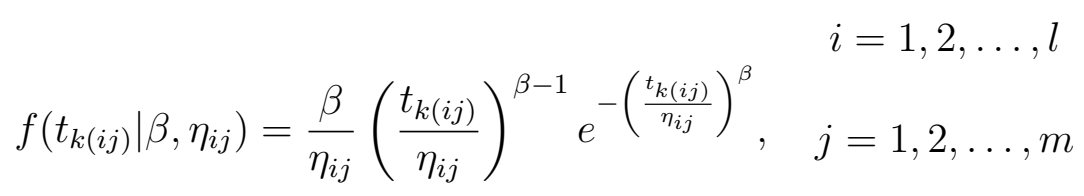

$$
\begin{aligned}
& k=1,2, \ldots, n
\end{aligned}
$$


where $\beta>0$ is the common shape parameter, and $\eta_{i j}>0$ is the scale parameter for test stand $j$ in block (batch) $i$. As a member of the log-location-scale family, the Weibull distribution can be reparameterized by $\log \left(\eta_{i j}\right)=\mu_{i j}=\mathbf{x}_{i j}^{T} \boldsymbol{\gamma}+\rho_{i}+\omega_{i j}$, where $\boldsymbol{\gamma}$ indicates the effect on the $\log$-scale parameter of treatment $\mathbf{x}_{i j}^{T}$. The random block effect, $\rho_{i}$, and the test stand effect, $\omega_{i j}$, are independent with $\rho_{i} \sim \operatorname{iidN}\left(0, \sigma_{\rho}^{2}\right)$ and $\omega_{i j} \sim \operatorname{iidN}\left(0, \sigma_{\omega}^{2}\right)$. It is appropriate to assume a common shape parameter across test stands, blocks, and treatments. Such an assumption is very reasonable as long as the failure mechanism is not changed by the applied treatment. Note that each block must be assigned to at least two test stands in order to separate the block and test stand effects.

\subsection{Two-Stage Method: Random Blocks with Subsampling}

Freeman and Vining (2010) introduces a two-stage method for analyzing right censored Weibull distributed reliability data with subsampling. The following two-stage method extends the method to the case with random blocks.

The first stage involves estimating the log-scale parameter $\mu_{i j}$ for each test stand and the pooled shape parameter $\beta$. The $\log$-likelihood for test stand $j$ in block $i$ is

$$
\mathcal{L}_{i j}\left(\beta, \mu_{i j} \mid \rho_{i}, \omega_{i j}\right)=\mathcal{C} \prod_{k=1}^{n}\left[f\left(t_{k(i j)}\right)\right]^{\delta_{k(i j)}}\left[1-F\left(t_{k(i j)}\right)\right]^{1-\delta_{k(i j)}}
$$

where $\delta_{k(i j)}=1$ if an item fails and $\delta_{k(i j)}=0$ if an item is censored. In addition, $f\left(t_{k(i j)}\right)$ is the PDF of the Weibull distribution and $F\left(t_{k(i j)}\right)$ is the cumulative distribution function 
(CDF) of the Weibull distribution. Meeker and Escobar (1998) discusses the impact of the constant $\mathcal{C}$ on the likelihood. The constant $\mathcal{C}$ reflects the type of censoring and sampling scheme. Since $\mathcal{C}$ is a constant that does not depend on $\beta, \mu_{i j}$ or the data, one can take $\mathcal{C}=1$ without loss of generality. The total log-likelihood conditional on $\boldsymbol{\rho}$ and $\boldsymbol{\omega}$ is

$$
\mathcal{L}\left(\beta, \mu_{11}, \ldots, \mu_{l m} \mid \boldsymbol{\rho}, \boldsymbol{\omega}\right)=\sum_{i=1}^{l} \sum_{j=1}^{m} \sum_{k=1}^{n}\left[\delta_{k(i j)} \log \left(\frac{\beta}{t_{k(i j)}}\right)+\delta_{k(i j)} z_{k(i j)}-e^{z_{k(i j)}}\right]
$$

where $z_{k(i j)}=\beta\left[\log \left(t_{k(i j)}\right)-\mu_{i j}\right]$. Standard statistical software packages, such as MINITAB and JMP, can estimate $\beta$ and each $\mu_{i j}=\log \left(\eta_{i j}\right)$.

The asymptotic variance-covariance matrix is

$$
\begin{aligned}
\hat{\Sigma} & =\left[\begin{array}{cccc}
\widehat{\operatorname{Var}}(\hat{\beta}) & \widehat{\operatorname{Cov}}\left(\hat{\beta}, \hat{\mu}_{11}\right) & \ldots & \widehat{\operatorname{Cov}}\left(\hat{\beta}, \hat{\mu}_{l m}\right) \\
\widehat{\operatorname{Cov}}\left(\hat{\beta}, \hat{\mu}_{11}\right) & \widehat{\operatorname{Var}}\left(\hat{\mu}_{11}\right) & \ldots & \vdots \\
\vdots & & \ddots & \widehat{\operatorname{Cov}}\left(\hat{\mu}_{l-1, m}, \hat{\mu}_{l m}\right) \\
\widehat{\operatorname{Cov}}\left(\hat{\beta}, \hat{\mu}_{l m}\right) & \ldots & \widehat{\operatorname{Cov}}\left(\hat{\mu}_{l-1, m}, \hat{\mu}_{l m}\right) & \widehat{\operatorname{Var}}\left(\hat{\mu}_{l m}\right)
\end{array}\right] \\
& =\left[\begin{array}{cccc}
-\frac{\partial^{2} \mathcal{L}\left(\hat{\beta}, \hat{\mu}_{11}, \ldots, \hat{\mu}_{l m}\right)}{\partial \beta^{2}} & -\frac{\partial^{2} \mathcal{L}\left(\hat{\beta}, \hat{\mu}_{11}, \ldots, \hat{\mu}_{l m}\right)}{\partial \beta \partial \mu_{11}} & \ldots & -\frac{\partial^{2} \mathcal{L}\left(\hat{\beta}, \hat{\mu}_{11}, \ldots, \hat{\mu}_{l m}\right)}{\partial \beta \partial \mu_{l m}} \\
-\frac{\partial^{2} \mathcal{L}\left(\hat{\beta}, \hat{\mu}_{11}, \ldots, \hat{\mu}_{l m}\right)}{\partial \beta \mu_{11}} & -\frac{\partial^{2} \mathcal{L}\left(\hat{\beta}, \hat{\mu}_{11}, \ldots, \hat{\mu}_{l m}\right)}{\partial \mu_{11}^{2}} & \ldots & \vdots \\
\vdots & & \ddots & -\frac{\partial^{2} \mathcal{L}\left(\hat{\beta}, \hat{\mu}_{11}, \ldots, \hat{\mu}_{l m}\right)}{\partial \mu_{l-1, m} \partial \mu_{l m}} \\
-\frac{\partial^{2} \mathcal{L}\left(\hat{\beta}, \hat{\mu}_{11}, \ldots, \hat{\mu}_{l m}\right)}{\partial \beta \partial \mu_{l m}} & \ldots & -\frac{\partial^{2} \mathcal{L}\left(\hat{\beta}, \hat{\mu}_{11}, \ldots, \hat{\mu}_{l m}\right)}{\partial \mu_{l-1, m} \partial \mu_{l m}} & -\frac{\partial^{2} \mathcal{L}\left(\hat{\beta}, \hat{\mu}_{11}, \ldots, \hat{\mu}_{l m}\right)}{\partial \mu_{l m}^{2}}
\end{array}\right] .
\end{aligned}
$$


Note that $-\frac{\partial^{2} \mathcal{L}\left(\hat{\beta}, \hat{\mu}_{11}, \ldots, \hat{\mu}_{l m}\right)}{\partial \beta^{2}}=\sum_{i=1}^{l} \sum_{j=1}^{m} \sum_{k=1}^{n}\left[\frac{\delta_{k(i j)}}{\hat{\beta}^{2}}+\left(\frac{z_{k(i j)}}{\hat{\beta}}\right)^{2} \exp \left(\hat{z}_{k(i j)}\right)\right],-\frac{\partial^{2} \mathcal{L}\left(\hat{\beta}, \hat{\mu}_{11}, \ldots, \hat{\mu}_{l m}\right)}{\partial \mu_{i j}^{2}}=$ $\sum_{k=1}^{n} \hat{\beta}^{2} \exp \left(\hat{z}_{k(i j)}\right),-\frac{\partial^{2} \mathcal{L}\left(\hat{\beta}, \hat{\mu}_{11}, \ldots, \hat{\mu}_{l m}\right)}{\partial \beta \partial \mu_{i j}}=\sum_{k=1}^{n}\left[\delta_{k(i j)}-\left(\hat{z}_{k(i j)}+1\right) \exp \left(\hat{z}_{k(i j)}\right)\right]$ and the second partial derivatives between all pairs of $\hat{\mu}_{i j}$ and $\hat{\mu}_{i^{\prime} j^{\prime}}$ is zero for all $i \neq i^{\prime}$ and $j \neq j^{\prime}$.

The second stage involves testing the effects of the factors. The stage 2 model is

$$
\hat{\boldsymbol{\mu}}=\boldsymbol{X} \gamma+Z \boldsymbol{\rho}+\boldsymbol{\omega}
$$

where $\hat{\boldsymbol{\mu}}$ is the $\log$ of the estimated scale parameters from stage $1, \gamma$ is the effect of the factors on the $\log$ of the estimated scale parameter, $\boldsymbol{Z}$ is the incidence matrix for the blocks, and $\boldsymbol{\rho}$ is the random block effects. We have $\boldsymbol{Z} \boldsymbol{\rho}+\boldsymbol{\omega} \sim M V N(\mathbf{0}, \boldsymbol{V})$, where $\boldsymbol{\rho}$ and $\boldsymbol{\omega}$ are independent and $\boldsymbol{V}$ represents the variance of the scale parameter estimates. Freeman and Vining (2010) notes that since the second derivatives between all pairs of $\hat{\mu}_{i j}$ and $\hat{\mu}_{i^{\prime} j^{\prime}}$ are zero it is appropriate to assume $\boldsymbol{V}=\left\langle\widehat{\operatorname{Var}}\left(\hat{\mu}_{i j}\right)\right\rangle$, where

$$
\begin{aligned}
\boldsymbol{V} & =\left\langle\widehat{\operatorname{Var}}\left(\hat{\mu}_{i j}\right)\right\rangle \\
& =\left[\begin{array}{cccc}
\widehat{\operatorname{Var}}\left(\hat{\mu}_{11}\right) & 0 & \ldots & 0 \\
0 & \widehat{\operatorname{Var}}\left(\hat{\mu}_{21}\right) & & \vdots \\
\vdots & & \ddots & 0 \\
0 & \ldots & 0 & \widehat{\operatorname{Var}}\left(\hat{\mu}_{l m}\right)
\end{array}\right] .
\end{aligned}
$$


The factor effects are estimated by $\hat{\boldsymbol{\gamma}}=\left(\boldsymbol{X}^{\prime} \boldsymbol{V}^{-1} \boldsymbol{X}\right)^{-1} \boldsymbol{V}^{-1} \boldsymbol{X}^{\prime} \boldsymbol{V}^{-1} \hat{\boldsymbol{\mu}}$. The stage 2 analysis can be performed using software packages such as MINITAB and JMP.

\subsection{An Illustrative Example}

To illustrate the two-stage method for analyzing a reliability experiment with random blocks and subsampling, consider an example in which an engineer wants to determine the effect of operating temperature on battery life. The batteries come from three batches. Due to time constraints the researchers decide to use type II censoring, which ensures that they observe a minimum of four failures for each temperature-batch combination. For each temperature and batch combination, the researchers place eight batteries in a chamber with the set temperature. Table 4.1 (Montgomery, pg. 165 altered) shows the first four failure times for each temperature and batch combination. The remaining four batteries for each combination represent censored observations. 
Table 4.1: Battery Life (in Hours)

\begin{tabular}{c|ccc}
\hline Batch & \multicolumn{3}{|c}{ Temperature $\left({ }^{\circ} \mathrm{F}\right)$} \\
\cline { 2 - 4 } & 15 & 70 & 125 \\
\hline 1 & 74 & 34 & 20 \\
& 130 & 40 & 58 \\
& 155 & 75 & 70 \\
& 180 & 80 & 82 \\
\hline 2 & 126 & 106 & 25 \\
& 150 & 115 & 45 \\
& 159 & 122 & 58 \\
& 188 & 136 & 70 \\
\hline 3 & 110 & 120 & 60 \\
& 138 & 139 & 82 \\
& 160 & 150 & 96 \\
& 168 & 174 & 104 \\
\hline
\end{tabular}

The stage 1 analysis estimates the common shape parameter and a different scale parameter for each temperature-batch combination. Table 4.2 shows the estimated scale parameter, $\log$ of estimated scale parameter, and estimated variance for each batch-temperature combination. The estimated shape parameter is $\hat{\beta}=4.03$, indicating failure due to product wear out. 
Table 4.2: MINITAB Stage One Analysis

\begin{tabular}{|c|c|c|c|c|}
\hline Batch & Temperature & $\hat{\eta}_{i j}$ & $\hat{\mu}_{i j}=\log \left(\hat{\eta}_{i j}\right)$ & $\widehat{\operatorname{Var}}\left(\hat{\mu}_{i j}\right)$ \\
\hline 1 & 15 & 197.79 & 5.287 & 0.0158 \\
1 & 70 & 87.98 & 4.477 & 0.0158 \\
1 & 125 & 89.85 & 4.498 & 0.0158 \\
2 & 15 & 208.88 & 5.342 & 0.0160 \\
2 & 70 & 153.56 & 5.034 & 0.0160 \\
2 & 125 & 76.28 & 4.334 & 0.0158 \\
3 & 15 & 189.22 & 5.243 & 0.0160 \\
3 & 70 & 193.85 & 5.267 & 0.0160 \\
3 & 125 & 116.05 & 4.754 & 0.0159 \\
\hline
\end{tabular}

Since the $\widehat{\operatorname{Var}}\left(\hat{\mu}_{i j}\right)$ for all $i j$ are similar, there is virtually no loss in using ordinary least squares. Table 4.3 contains the results of the stage 2 analysis. The stage 2 analysis indicates that operating temperature has a significant effect on product wear out.

Table 4.3: MINITAB Stage Two Analysis

\begin{tabular}{c|cccc} 
Source & DF & MS & F & P \\
\hline Temperature & 1 & 0.8704 & 16.96 & 0.009 \\
Block & 2 & 0.0839 & 1.63 & 0.284 \\
Error & 5 & 0.05132 & & \\
\hline \multicolumn{4}{c}{$\hat{\sigma}_{\omega}^{2}=0.05132$} & $\hat{\sigma}_{\rho}^{2}=0.01086$ \\
\hline
\end{tabular}

The random block error is calculated by $\hat{\sigma}_{\rho}^{2}=\frac{M S_{\text {Block }}-M S_{\text {Error }}}{3}=0.01086$.

\subsection{Simulation Study}

The performance of the two-stage method was examined and compared with both the traditional analysis (assuming a completely randomized design) and the traditional analysis 
with fixed block effects using 10,000 simulations in SAS. The simulation study evaluates the bias and variability of model parameters as well as the type I and type II error rates. The simulation scenario involved failure time data with random blocks and subsampling. The simulations contained one factor $x$ (with two levels denoted by -1 and 1 ) that represented operating conditions. The simulation scenario included four batches of materials, which are the random blocks. Each batch provided materials for two test stands (one for each of the two operating conditions), with each test stand containing $n$ items. The simulation records the first $r$ failures for each test stand, employing type II censoring. The failure times follow a Weibull distribution with shape parameters of $\beta=5,3,1$, and 0.5 , and $\log$-scale parameter $\log \left(\eta_{i j}\right)=\mu_{i j}=\gamma_{0}+\gamma_{1} x_{i}+\rho_{i}+\omega_{i j}$ where $\rho_{i} \sim N\left(0, \sigma_{\rho}^{2}\right)$ and $\omega_{i j} \sim N\left(0, \sigma_{\omega}^{2}\right)$. Table 4.4 provides an outline of the simulations performed.

Table 4.4: Outline of Simulations

\begin{tabular}{|c|c|c|c|c|}
\hline Simulation & $n$ & $r$ & & Results \\
\hline 1 & 8 & 4 & Type I Error (0.05) & Table 4.5 \\
2 & 8 & 4 & Power (0.50) & Table 4.6 \\
3 & 8 & 4 & Power (0.90) & Table 4.7 \\
4 & 16 & 8 & Type I Error (0.05) & Table 4.8 \\
5 & 16 & 8 & Power (0.50) & Table 4.9 \\
6 & 16 & 8 & Power (0.90) & Table 4.10 \\
7 & 16 & 12 & Type I Error (0.05) & Table 4.11 \\
8 & 16 & 12 & Power (0.50) & Table 4.12 \\
9 & 16 & 12 & Power (0.90) & Table 4.13 \\
\hline
\end{tabular}

The first set of simulations examines the type I empirical error rate for a nominal value of $\alpha=0.05$. For the first set of simulations the following values were used $n=8, r=4$, 
$\gamma_{0}=15, \gamma_{1}=0, \sigma_{\rho}=2$ and $\sigma_{\omega}=1$. A value of $\gamma_{1}=0$ means that factor $x$ (operating condition) does not effect the failure time. Table 4.5 shows the results of the simulations.

Table 4.5: Empirical Error Simulation Results: $n=8, r=4$

\begin{tabular}{|c|c|c|c|c|c|c|c|}
\hline & \multirow[b]{2}{*}{ Actual } & $\gamma_{0}$ & $\gamma_{1}$ & $\beta$ & $\sigma_{\rho}$ & $\sigma_{\omega}$ & $\alpha$ \\
\hline & & 15 & 0 & & 2 & 1 & 0.05 \\
\hline \multirow[t]{6}{*}{$\beta=5$} & Two-Stage Method & 14.9306 & -0.0020 & 6.6786 & 1.7682 & 0.9175 & 0.0477 \\
\hline & & $(0.0106)$ & $(0.0035)$ & $(0.0133)$ & $(0.0092)$ & $(0.0038)$ & $(0.0021)$ \\
\hline & Traditional Analysis with Blocks & 15.4089 & -0.0026 & 2.2140 & - & - & 0.5267 \\
\hline & & $(0.0214)$ & $(0.0037)$ & $(0.0082)$ & - & - & $(0.0050)$ \\
\hline & Traditional Analysis & 16.7902 & 0.0032 & 0.7102 & - & - & 0.2512 \\
\hline & & $(0.0130)$ & $(0.0046)$ & $(0.0031)$ & - & - & $(0.0043)$ \\
\hline \multirow[t]{6}{*}{$\beta=3$} & Two-Stage Method & 14.8930 & -0.0029 & 4.0081 & 1.7612 & 0.9304 & 0.0528 \\
\hline & & $(0.0107)$ & $(0.0036)$ & $(0.0080)$ & $(0.0093)$ & $(0.0039)$ & $(0.0022)$ \\
\hline & Traditional Analysis with Blocks & 15.3473 & -0.0018 & 1.8366 & - & - & 0.4819 \\
\hline & & $(0.0217)$ & $(0.0037)$ & $(0.0057)$ & - & - & $(0.0050)$ \\
\hline & Traditional Analysis & 16.6872 & 0.0005 & 0.6756 & - & - & 0.2340 \\
\hline & & $(0.0132)$ & $(0.0046)$ & $(0.0027)$ & - & - & $(0.0042)$ \\
\hline \multirow[t]{6}{*}{$\beta=1$} & Two-Stage Method & 14.7446 & -0.0067 & 1.3346 & 1.7705 & 1.0317 & 0.0524 \\
\hline & & $(0.0108)$ & $(0.0040)$ & $(0.0026)$ & $(0.0096)$ & $(0.0043)$ & $(0.0022)$ \\
\hline & Traditional Analysis with Blocks & 15.0658 & -0.0063 & 0.9749 & - & - & 0.3083 \\
\hline & & $(0.0220)$ & $(0.0041)$ & $(0.0020)$ & - & - & $(0.0046)$ \\
\hline & Traditional Analysis & 16.3180 & -0.0054 & 0.5237 & - & - & 0.1643 \\
\hline & & $(0.0129)$ & $(0.0049)$ & $(0.0015)$ & - & - & $(0.0037)$ \\
\hline \multirow[t]{6}{*}{$\beta=0.5$} & Two-Stage Method & 14.4750 & -0.0043 & 0.6668 & 1.7155 & 1.3172 & 0.0499 \\
\hline & & $(0.0113)$ & $(0.0052)$ & $(0.0013)$ & $(0.0106)$ & $(0.0055)$ & $(0.0022)$ \\
\hline & Traditional Analysis with Blocks & 14.8085 & -0.0041 & 0.5547 & - & - & 0.1864 \\
\hline & & $(0.0227)$ & $(0.0052)$ & $(0.0010)$ & - & - & $(0.0039)$ \\
\hline & Traditional Analysis & 15.8873 & -0.0084 & 0.3829 & - & - & 0.1116 \\
\hline & & $(0.0131)$ & $(0.0058)$ & $(0.0009)$ & - & - & $(0.0031)$ \\
\hline
\end{tabular}

The estimates for $\gamma_{0}, \gamma_{1}, \beta, \sigma_{\rho}$ and $\sigma_{\omega}$ are the mean of the estimates for the 10,000 simulations. Simulation error is shown in parentheses.

Table 4.5 shows that the two-stage analysis preserves the nominal type I error rate of 0.05 . Although the traditional analysis has a nominal type I error rate of 0.05 , the empirical error is much larger. The empirical error ranges from 0.1116 to 0.2512 and appears to depend on the value of $\beta$. The larger the value of $\beta$ the greater the empirical error deviates from the nominal type I error rate. The traditional analysis with blocks produces an even more inflated empirical error than the traditional analysis. The empirical error for the traditional 
analysis with blocks ranges from 0.1864 to 0.5267 depending on the value of $\beta$. This inflation makes using the traditional analysis and traditional analysis with blocks dangerous, since these methods underestimate the experimental error which leads to false positives for the significance of $x$. All three methods provide reasonable estimates of $\gamma_{0}$ and $\gamma_{1}$. The two-stage method consistently overestimates $\beta$ by a factor of 1.33 . Both the traditional analysis and the traditional analysis with blocks provide estimates that appear to depend on $\beta$. Whereas the traditional analysis consistently underestimates $\beta$, the traditional analysis with blocks underestimates $\beta$ when $\beta=3$ or $\beta=5$ and produces estimates close to the nominal values when $\beta=1$ and $\beta=0.5$. The two-stage method underestimates $\sigma_{\rho}$, and the estimates of $\sigma_{\omega}$ seem to be inversely related to $\beta$. When $\beta=5$ the two-stage method underestimates $\sigma_{\omega}$, while when $\beta=0.5$ the two-stage method overestimates $\sigma_{\omega}$.

The second set of simulations compares the two-stage analysis, traditional analysis and traditional analysis with blocks for a nominal power of 0.50 with a nominal type I error rate of 0.05 . This set of simulations uses the following values $n=8, r=4, \gamma_{0}=15, \gamma_{1}=1.01$, $\sigma_{\rho}=2$ and $\sigma_{\omega}=1$, where $\gamma_{1}$ is set to obtain the desired nominal power. Under the assumption that $\gamma_{1}=0, P\left(F^{*}>F_{0.95,1,3, \lambda=0}=10.12796\right)=0.05$ where $F^{*}$ is the observed $F$ statistic in stage 2 of the two-stage analysis. For this design the noncentrality parameter is $\lambda=\frac{8 \gamma_{1}^{2}}{\sigma_{\omega}^{2}}$. To find the value of $\gamma_{1}$ that will give a nominal power of 0.50 , first find the value of $\lambda$ such that $P\left(F^{*}>10.12796 \mid \lambda\right)=0.50$. Solving for $\lambda$ gives $\lambda=8.225$. Next, substitute $\lambda$ into the equation $\lambda=8 \gamma_{1}^{2}$. Solving for $\gamma_{1}$ produces $\gamma_{1}=1.01$ (and -1.01). Table 4.6 shows the results of the simulations. 
Table 4.6: Simulation Results Power 0.50: $n=8, r=4$

\begin{tabular}{|c|c|c|c|c|c|c|c|}
\hline & \multirow[b]{2}{*}{ Actual } & $\gamma_{0}$ & $\gamma_{1}$ & \multirow[t]{2}{*}{$\beta$} & \multirow{2}{*}{$\frac{\sigma_{\rho}}{2}$} & $\sigma_{\omega}$ & Power \\
\hline & & 15 & 1.01 & & & 1 & 0.50 \\
\hline \multirow[t]{6}{*}{$\beta=5$} & Two-Stage Method & 14.9380 & 1.0087 & 6.6717 & 1.7753 & 0.9169 & 0.4941 \\
\hline & & $(0.0107)$ & $(0.0035)$ & $(0.0131)$ & $(0.0092)$ & $(0.0038)$ & $(0.0050)$ \\
\hline & Traditional Analysis with Blocks & 15.3861 & 1.0076 & 2.2183 & - & - & 0.9797 \\
\hline & & $(0.0215)$ & $(0.0037)$ & $(0.0083)$ & - & - & $(0.0014)$ \\
\hline & Traditional Analysis & 16.8033 & 1.0076 & 0.7056 & - & - & 0.8081 \\
\hline & & $(0.0131)$ & $(0.0045)$ & $(0.0030)$ & - & - & $(0.0039)$ \\
\hline \multirow[t]{6}{*}{$\beta=3$} & Two-Stage Method & 14.9127 & 1.0079 & 4.0156 & 1.7833 & 0.9333 & 0.4818 \\
\hline & & $(0.0106)$ & $(0.0036)$ & $(0.0079)$ & $(0.0094)$ & $(0.0039)$ & $(0.0050)$ \\
\hline & Traditional Analysis with Blocks & 15.2880 & 1.0074 & 1.8327 & - & - & 0.9754 \\
\hline & & $(0.0214)$ & $(0.0037)$ & $(0.0057)$ & - & - & $(0.0015)$ \\
\hline & Traditional Analysis & 16.7286 & 1.0020 & 0.6675 & - & - & 0.7967 \\
\hline & & $(0.0131)$ & $(0.0045)$ & $(0.0027)$ & - & - & $(0.0040)$ \\
\hline \multirow[t]{6}{*}{$\beta=1$} & Two-Stage Method & 14.7310 & 1.0080 & 1.3333 & 1.7670 & 1.0328 & 0.4189 \\
\hline & & $(0.0108)$ & $(0.0040)$ & $(0.0026)$ & $(0.0097)$ & $(0.0043)$ & $(0.0049)$ \\
\hline & Traditional Analysis with Blocks & 15.0918 & 1.0073 & 0.9744 & - & - & 0.9168 \\
\hline & & $(0.0219)$ & $(0.0041)$ & $(0.0020)$ & - & - & $(0.0028)$ \\
\hline & Traditional Analysis & 16.3074 & 1.0088 & 0.5237 & - & - & 0.7151 \\
\hline & & $(0.0130)$ & $(0.0049)$ & $(0.0015)$ & - & - & $(0.0045)$ \\
\hline \multirow[t]{6}{*}{$\beta=0.5$} & Two-Stage Method & 14.4665 & 1.0166 & 0.6659 & 1.7268 & 1.3182 & 0.2904 \\
\hline & & $(0.0114)$ & $(0.0052)$ & $(0.0013)$ & $(0.0105)$ & $(0.0054)$ & $(0.0045)$ \\
\hline & Traditional Analysis with Blocks & 14.7890 & 1.0175 & 0.5534 & - & - & 0.7133 \\
\hline & & $(0.0227)$ & $(0.0052)$ & $(0.0010)$ & - & - & $(0.0045)$ \\
\hline & Traditional Analysis & 15.8803 & 1.0186 & 0.3815 & - & - & 0.5548 \\
\hline & & $(0.0130)$ & $(0.0058)$ & $(0.0008)$ & - & - & $(0.0050)$ \\
\hline
\end{tabular}

The estimates for $\gamma_{0}, \gamma_{1}, \beta, \sigma_{\rho}$ and $\sigma_{\omega}$ are the mean of the estimates for the 10,000 simulations. Simulation error is shown in parentheses.

Table 4.6 indicates that the empirical power of the two-stage method is close to the nominal power of 0.50 for $\beta=5$ and $\beta=3$. However, for $\beta=1$ and $\beta=0.5$ the empirical power drops. The power appears to be related to the value of $\beta$, where the power decreases as $\beta$ decreases. The traditional analysis overstates the significance of $x$ in general; however, for $\beta=0.5$ the empirical power comes close to the nominal power. The traditional analysis with blocks consistently overstates the significance of $x$. The estimates of the parameters follow the same pattern as in the empirical error simulations. 
The final set of simulations using $n=8$ and $r=4$ compares the two-stage analysis, traditional analysis and traditional analysis with blocks for a nominal power of 0.90 and a nominal type I error rate of 0.05 . The simulations use the following values: $\gamma_{0}=15, \gamma_{1}=1.77, \sigma_{\rho}=2$ and $\sigma_{\omega}=1$. Table 4.7 shows the results of the simulations.

Table 4.7: Simulation Results Power 0.90: $n=8, r=4$

\begin{tabular}{|c|c|c|c|c|c|c|c|}
\hline & & $\gamma_{0}$ & $\gamma_{1}$ & $\beta$ & $\sigma_{\rho}$ & $\sigma_{\omega}$ & Power \\
\hline & Actual & 15 & 1.77 & & 2 & 1 & 0.90 \\
\hline \multirow[t]{6}{*}{$\beta=5$} & Two-Stage Method & 14.9549 & 1.7731 & 6.7023 & 1.7883 & 0.9165 & 0.9051 \\
\hline & & $(0.0106)$ & $(0.0035)$ & $(0.0135)$ & $(0.0093)$ & $(0.0038)$ & $(0.0029)$ \\
\hline & Traditional Analysis with Blocks & 15.4465 & 1.7743 & 2.2188 & - & - & 1.0000 \\
\hline & & $(0.0217)$ & $(0.0037)$ & $(0.0082)$ & - & - & $(0.0000)$ \\
\hline & Traditional Analysis & 16.8316 & 1.7742 & 0.7053 & - & - & 0.9862 \\
\hline & & $(0.0132)$ & $(0.0045)$ & $(0.0031)$ & - & - & $(0.0012)$ \\
\hline \multirow[t]{6}{*}{$\beta=3$} & Two-Stage Method & 14.9169 & 1.7624 & 3.9993 & 1.7623 & 0.9238 & 0.8982 \\
\hline & & $(0.0106)$ & $(0.0035)$ & $(0.0078)$ & $(0.0093)$ & $(0.0038)$ & $(0.0030)$ \\
\hline & Traditional Analysis with Blocks & 15.3373 & 1.7629 & 1.8416 & - & - & 0.9998 \\
\hline & & $(0.0213)$ & $(0.0036)$ & $(0.0056)$ & - & - & $(0.0001)$ \\
\hline & Traditional Analysis & 16.7040 & 1.7600 & 0.6753 & - & - & 0.9840 \\
\hline & & $(0.0129)$ & $(0.0045)$ & $(0.0027)$ & - & - & $(0.0013)$ \\
\hline \multirow[t]{6}{*}{$\beta=1$} & Two-Stage Method & 14.7289 & 1.7731 & 1.3363 & 1.7392 & 1.0376 & 0.8340 \\
\hline & & $(0.0107)$ & $(0.0040)$ & $(0.0026)$ & $(0.0097)$ & $(0.0043)$ & $(0.0037)$ \\
\hline & Traditional Analysis with Blocks & 15.0838 & 1.7739 & 0.9738 & - & - & 0.9996 \\
\hline & & $(0.0215)$ & $(0.0041)$ & $(0.0020)$ & - & - & $(0.0002)$ \\
\hline & Traditional Analysis & 16.2888 & 1.7702 & 0.5275 & - & - & 0.9725 \\
\hline & & $(0.0128)$ & $(0.0049)$ & $(0.0016)$ & - & - & $(0.0016)$ \\
\hline \multirow[t]{6}{*}{$\beta=0.5$} & Two-Stage Method & 14.4971 & 1.7692 & 0.6665 & 1.7352 & 1.3163 & 0.6500 \\
\hline & & $(0.0115)$ & $(0.0052)$ & $(0.0013)$ & $(0.0105)$ & $(0.0054)$ & $(0.0048)$ \\
\hline & Traditional Analysis with Blocks & 14.8544 & 1.7691 & 0.5542 & - & - & 0.9750 \\
\hline & & $(0.0230)$ & $(0.0052)$ & $(0.0010)$ & - & - & $(0.0016)$ \\
\hline & Traditional Analysis & 15.9127 & 1.7673 & 0.3818 & - & - & 0.9038 \\
\hline & & $(0.0132)$ & $(0.0058)$ & $(0.0008)$ & - & - & $(0.0029)$ \\
\hline
\end{tabular}

The estimates for $\gamma_{0}, \gamma_{1}, \beta, \sigma_{\rho}$ and $\sigma_{\omega}$ are the mean of the estimates for the 10,000 simulations. Simulation error is shown in parentheses.

Table 4.7 shows that the empirical power simulations for a nominal power of 0.90 follow the same pattern as the simulations with a nominal power of 0.50 .

In addition to the above simulations, simulations were performed to examine the effect of the 
number of subsampling units on the power and bias of $\beta$. The following series of simulations uses 16 observational units on each test stand with 8 units failing. All other parameters are the same as in the previous series of simulations. Table 4.8 shows the simulation results for the empirical error.

Table 4.8: Empirical Error Rate Simulation Results: $n=16, r=8$

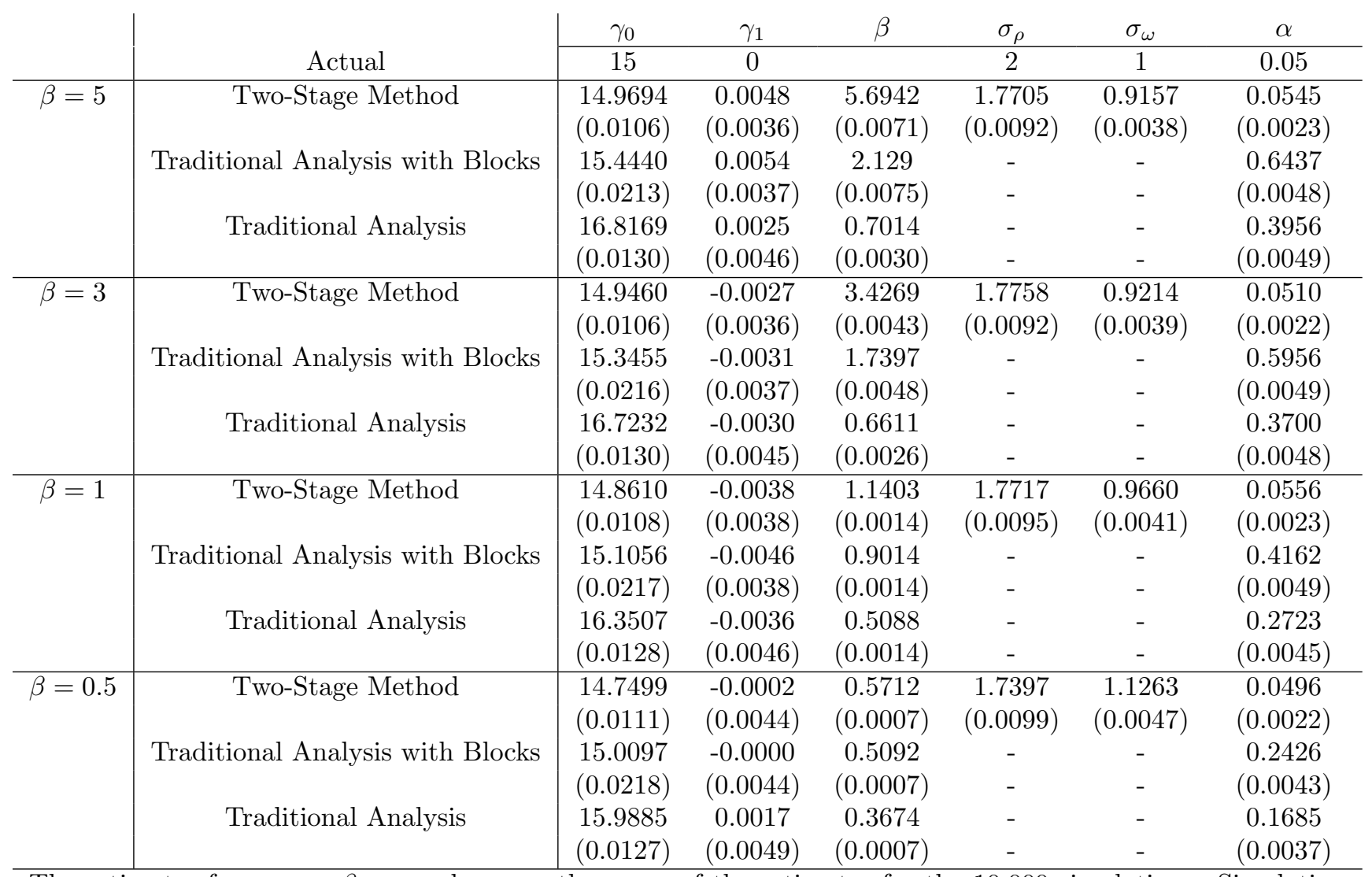

The estimates for $\gamma_{0}, \gamma_{1}, \beta, \sigma_{\rho}$ and $\sigma_{\omega}$ are the mean of the estimates for the 10,000 simulations. Simulation error is shown in parentheses.

Table 4.8 shows results that are similar to those of the empirical error simulations with $n=8$ and 4 failures for each test stand. However, note that the two-stage method overestimates $\beta$ by a factor of approximately 1.14 instead of 1.33. In addition, the empirical error for the traditional analysis and traditional analysis with blocks is slightly more inflated than in the 
previous case.

Table 4.9 displays the simulation results for a nominal power of 0.50 for $n=16$ with 8 failures per test stand.

Table 4.9: Simulation Results Power 0.50: $n=16, r=8$

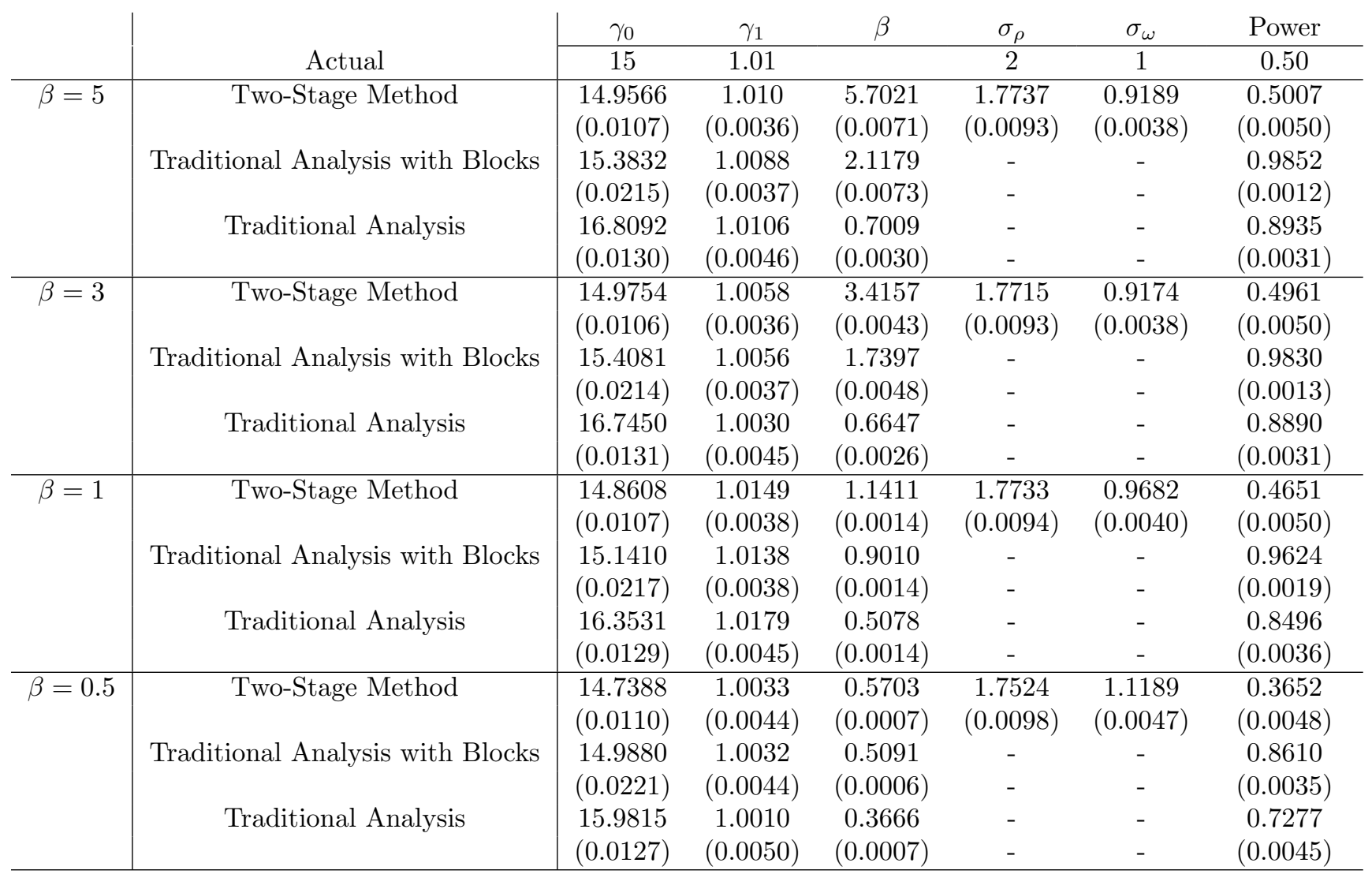

The estimates for $\gamma_{0}, \gamma_{1}, \beta, \sigma_{\rho}$ and $\sigma_{\omega}$ are the mean of the estimates for the 10,000 simulations. Simulation error is shown in parentheses.

The bias of $\beta$ for the two-stage method is the same as in Table 4.8. The power for the traditional analysis and traditional analysis with blocks is slightly more inflated than in Table 4.6. The two-stage method underestimates the power for $\beta=1$ and $\beta=0.5$; however, not as much as in Table 4.6. 
Table 4.10 shows the simulation results for the case of a nominal power of 0.90 with $n=16$ and 8 failures per test stand.

Table 4.10: Simulation Results Power 0.90: $n=16, r=8$

\begin{tabular}{|c|c|c|c|c|c|c|c|}
\hline & \multirow[b]{2}{*}{ Actual } & $\gamma_{0}$ & $\gamma_{1}$ & $\beta$ & \multirow{2}{*}{$\frac{\sigma_{\rho}}{2}$} & \multirow{2}{*}{$\frac{\sigma_{\omega}}{1}$} & \multirow{2}{*}{$\begin{array}{c}\text { Power } \\
0.90\end{array}$} \\
\hline & & 15 & 1.77 & & & & \\
\hline \multirow{6}{*}{$\beta=5$} & Two-Stage Method & 14.9727 & 1.7743 & 5.7037 & 1.7851 & 0.9123 & 0.9061 \\
\hline & & $(0.0105)$ & $(0.0035)$ & $(0.0072)$ & $(0.0092)$ & $(0.0039)$ & $(0.0029)$ \\
\hline & Traditional Analysis with Blocks & 15.4404 & 1.7744 & 2.1385 & - & - & 1.0000 \\
\hline & & $(0.02111)$ & $(0.0037)$ & $(0.0075)$ & - & - & $(0)$ \\
\hline & Traditional Analysis & 16.8291 & 1.7752 & 0.6968 & - & - & 0.9958 \\
\hline & & $(0.0129)$ & $(0.0045)$ & $(0.0029)$ & - & - & $(0.0006)$ \\
\hline \multirow[t]{6}{*}{$\beta=3$} & Two-Stage Method & 14.9345 & 1.7719 & 3.4209 & 1.7799 & 0.9222 & 0.9026 \\
\hline & & $(0.0106)$ & $(0.0035)$ & $(0.0043)$ & $(0.0093)$ & $(0.0038)$ & $(0.0030)$ \\
\hline & Traditional Analysis with Blocks & 15.3501 & 1.7717 & 1.7356 & - & - & 1.0000 \\
\hline & & $(0.0215)$ & $(0.0036)$ & $(0.0048)$ & - & - & $(0)$ \\
\hline & Traditional Analysis & 16.7093 & 1.7707 & 0.6605 & - & - & 0.9952 \\
\hline & & $(0.0130)$ & $(0.0045)$ & $(0.0025)$ & - & - & $(0.0007)$ \\
\hline \multirow[t]{6}{*}{$\beta=1$} & Two-Stage Method & 14.8718 & 1.7653 & 1.1403 & 1.7721 & 0.9731 & 0.8681 \\
\hline & & $(0.0107)$ & $(0.0038)$ & $(0.0014)$ & $(0.0094)$ & $(0.0040)$ & $(0.0034)$ \\
\hline & Traditional Analysis with Blocks & 15.1526 & 1.7652 & 0.8987 & - & - & 0.9999 \\
\hline & & $(0.0216)$ & $(0.0038)$ & $(0.0014)$ & - & - & $(0.0001)$ \\
\hline & Traditional Analysis & 16.3649 & 1.7668 & 0.5068 & - & - & 0.9937 \\
\hline & & $(0.0128)$ & $(0.0045)$ & $(0.0014)$ & - & - & $(0.0008)$ \\
\hline \multirow[t]{6}{*}{$\beta=0.5$} & Two-Stage Method & 14.7431 & 1.7712 & 0.5695 & 1.7609 & 1.1203 & 0.7739 \\
\hline & & $(0.0108)$ & $(0.0043)$ & $(0.0007)$ & $(0.0098)$ & $(0.0047)$ & $(0.0042)$ \\
\hline & Traditional Analysis with Blocks & 15.0133 & 1.7710 & 0.5086 & - & - & 0.9984 \\
\hline & & $(0.0217)$ & $(0.0043)$ & $(0.0007)$ & - & - & $(0.0004)$ \\
\hline & Traditional Analysis & 15.9925 & 1.7731 & 0.3654 & - & - & 0.9798 \\
\hline & & $(0.0125)$ & $(0.0049)$ & $(0.0007)$ & - & - & $(0.0021)$ \\
\hline
\end{tabular}

The estimates for $\gamma_{0}, \gamma_{1}, \beta, \sigma_{\rho}$ and $\sigma_{\omega}$ are the mean of the estimates for the 10,000 simulations. Simulation error is shown in parentheses.

The results of Table 4.10 are similar to those of Table 4.9 .

The next series of simulations uses the same parameter values as before, but has 16 observational units and 12 failures per test stand. Table 4.11 displays the simulation results for the empirical error. 
Table 4.11: Empirical Error Rate Simulation Results: $n=16, r=12$

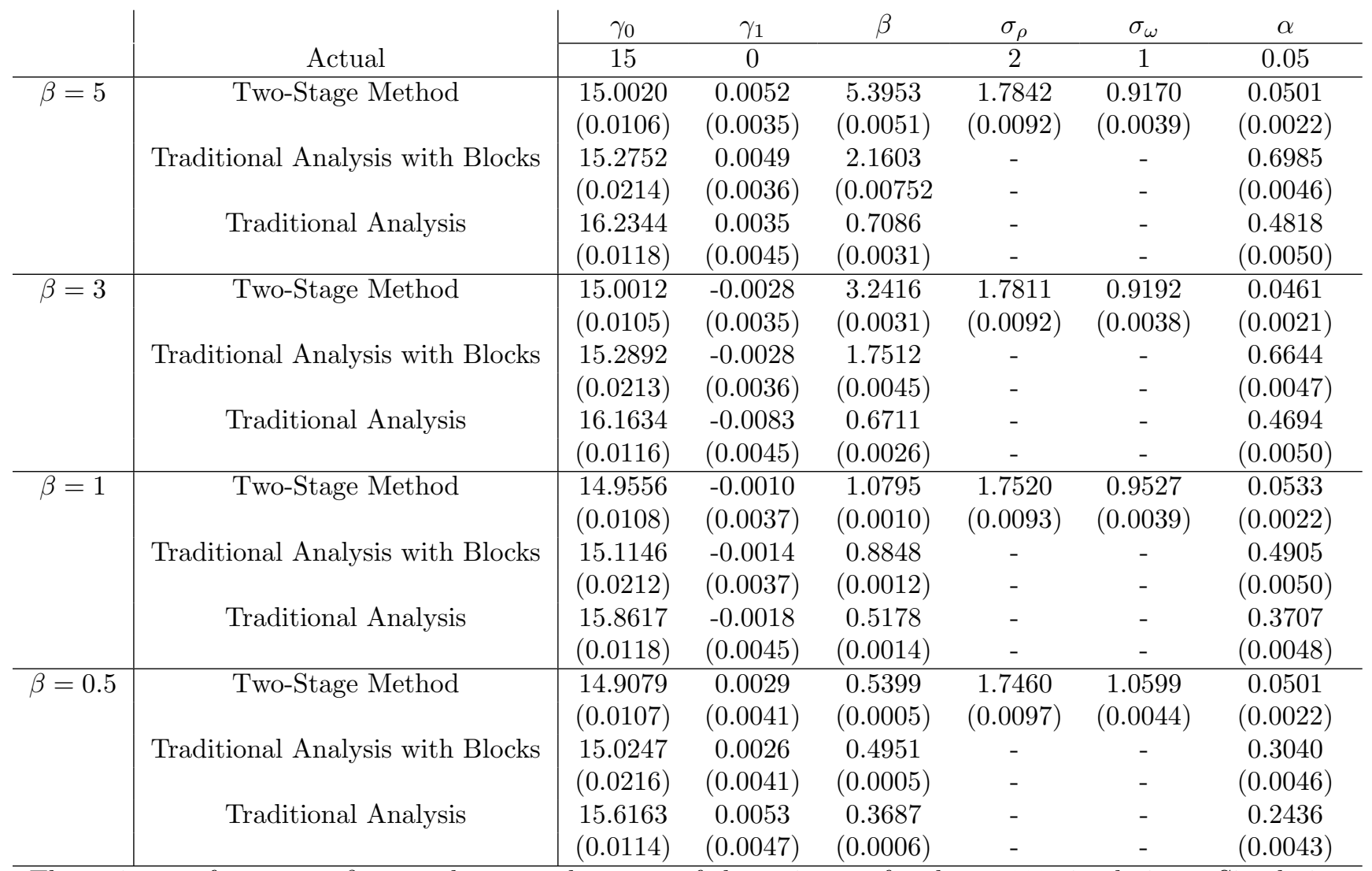

The estimates for $\gamma_{0}, \gamma_{1}, \beta, \sigma_{\rho}$ and $\sigma_{\omega}$ are the mean of the estimates for the 10,000 simulations. Simulation error is shown in parentheses.

The results in Table 4.11 are similar to those of Tables 4.5 and 4.8. The two-stage method's estimate of $\beta$ comes closer the the nominal value, overestimating $\beta$ by a factor of 1.08 . The test stand error, $\sigma_{\omega}$ becomes less inflated for $\beta=0.5$. In addition, the traditional analysis and traditional analysis with blocks have empirical errors that are even more inflated than in Table 4.8.

Table 4.12 gives the simulation results for a nominal power of 0.50 with $n=16$ and 12 failures per test stand. 
Table 4.12: Simulation Results Power 0.50: $n=16, r=12$

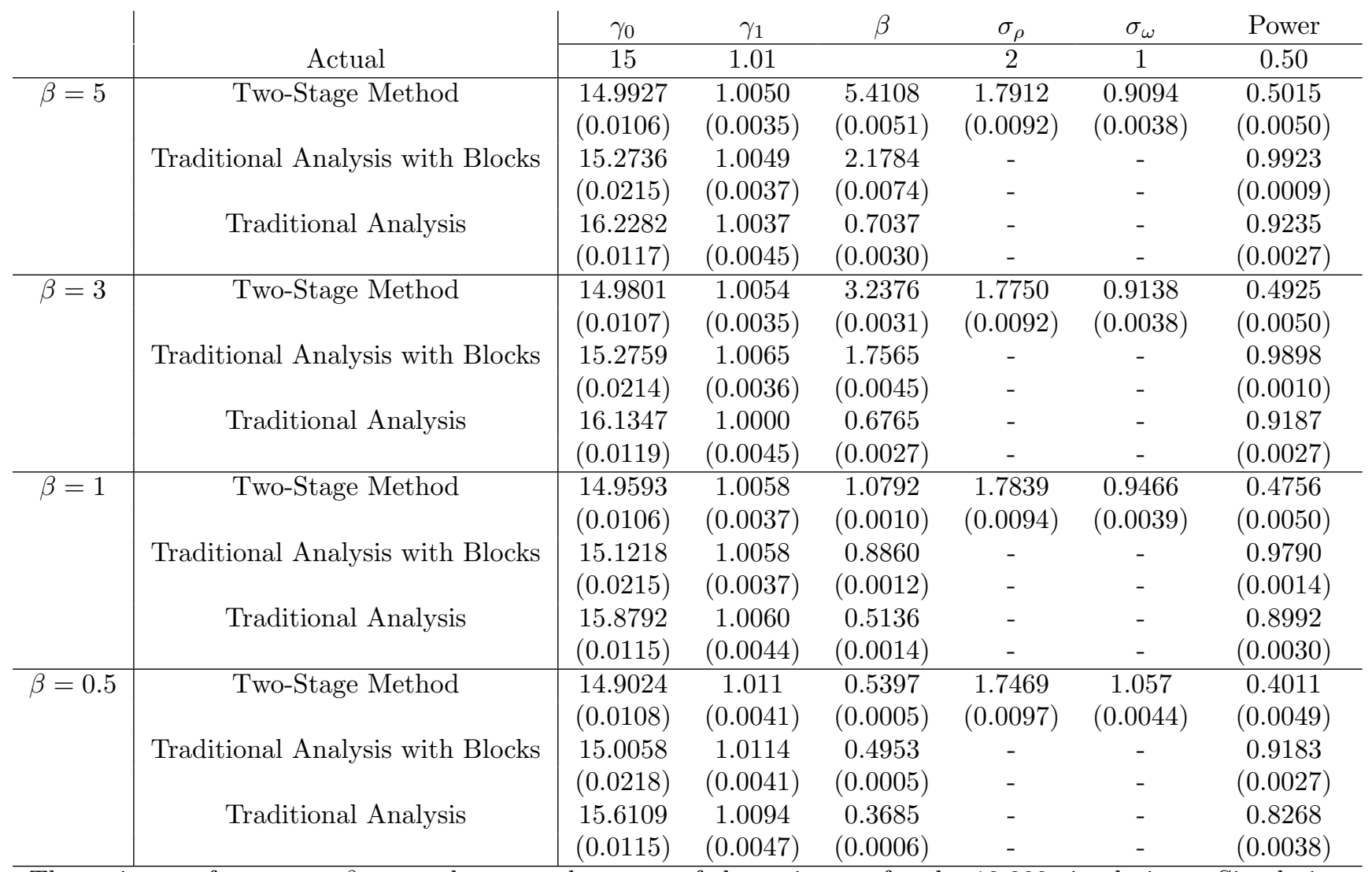

The estimates for $\gamma_{0}, \gamma_{1}, \beta, \sigma_{\rho}$ and $\sigma_{\omega}$ are the mean of the estimates for the 10,000 simulations. Simulation error is shown in parentheses.

The bias of $\beta$ for the two-stage method is the same as in Table 4.11. Along with Tables 4.6 and 4.9, Table 4.12 indicates that the power of the two-stage method approaches the nominal power as the number of failures increases.

Table 4.13 displays the simulations results for $n=16$ and 12 failures with a nominal power of 0.90 . 
Table 4.13: Simulation Results Power 0.90: $n=16, r=12$

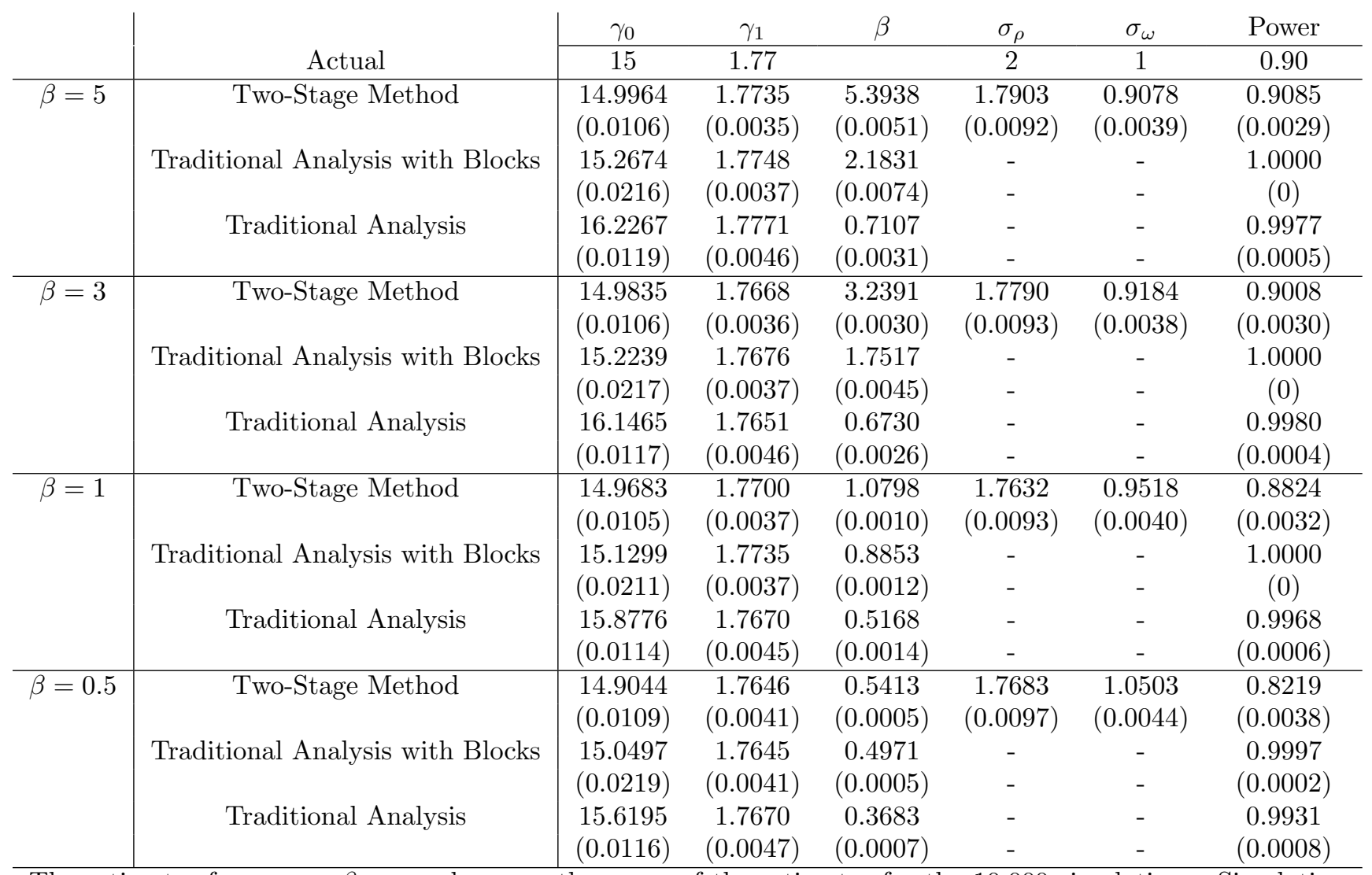

The estimates for $\gamma_{0}, \gamma_{1}, \beta, \sigma_{\rho}$ and $\sigma_{\omega}$ are the mean of the estimates for the 10,000 simulations. Simulation error is shown in parentheses.

These simulation results show the same patterns as the previous simulations. Although the nominal power is 0.90 , the traditional analysis and the traditional analysis with blocks produce empirical powers of approximately 100\%. In general, the two-stage method's empirical power is close to the nominal power for $\beta=5$ and $\beta=3$. For $\beta=1$ and $\beta=0.5$ the two-stage method approaches the nominal power as the number of failures per test stand increases.

In addition to figures that visually depict the information contained in the previous tables, 
Appendix A also contains tables summarizing simulation results for a nominal power of 0.80 .

\subsection{Conclusions for Two-Stage Random Block Analy- sis}

The two-stage method offers practitioners an easy way to analyze life data with random blocks with subsampling in standard statistical software packages such as MINITAB and JMP. Unfortunately, the two-stage method may produce biased estimates of the shape parameter and variance components. However, it may be possible to find a bias correction factor that would allow one to obtain an unbiased estimate of $\beta$. For small values of $\beta$ the two-stage method may not have the power to detect the significance of a factor if there are few failures. However, the two-stage method performs better than the currently used traditional analysis, which assumes a completely randomized design, and the traditional analysis with blocks as these methods have inflated type I error rates and inflated power. Furthermore, as the number of failures per test stand increases, the two-stage method produces less biased estimates of $\beta$ and empirical powers which approach the nominal power. A more sophisticated method introduced in Chapter 5 will analyze the data in a single step, with the shape parameter, factor effects and variance components estimated simultaneously. 


\section{Chapter 5}

\section{Joint-Likelihood Analysis}

\section{$5.1 \quad$ Introduction}

As discussed in the previous chapter, one limitation of the two-stage method is that the

covariances between $\hat{\beta}$ and the parameters estimated in stage $2\left(\hat{\gamma}_{0}, \hat{\gamma}_{1}, \hat{\sigma}_{\rho}\right.$ and $\left.\hat{\sigma}_{\omega}\right)$ are unknown. This means that inferences cannot easily be performed on certain functions of the parameters (such as percentiles), although point estimates can still be obtained. This chapter proposes a more rigorous analysis based on the joint-likelihood of $\beta, \gamma_{0}, \gamma_{1}, \sigma_{\rho}$ and $\sigma_{\omega}$ that allows inference on all functions of the parameters. This chapter also defines the unconditional likelihood of a reliability experiment with random blocks, subsampling and right censored data. This likelihood involves two intractable integrals, thus numerical methods are required. Two iterations of Gauss-Hermite quadrature are performed giving a 
closed form approximate likelihood. This likelihood allows maximum likelihood estimates to be obtained and the derivation of the variance-covariance matrix. Next, inferences based on the likelihood and normal distribution are outlined. This more rigorous method is illustrated using the battery life example from Chapter 4 .

\subsection{The Model}

This chapter uses the same experimental setup as in the previous chapter. We have

$$
\begin{gathered}
f_{1}\left(t_{k(i j)} \mid \beta, \eta_{i j}, \rho_{i}, \omega_{i j}\right)=\frac{\beta}{\eta_{i j}}\left(\frac{t_{k(i j)}}{\eta_{i j}}\right)^{\beta-1} e^{-\left(\frac{t_{k(i j)}}{\eta_{i j}}\right)^{\beta}} \\
F_{1}\left(t_{k(i j)} \mid \beta, \eta_{i j}, \rho_{i}, \omega_{i j}\right)=1-\exp \left[-\left(\frac{t_{k(i j)}}{\eta_{i j}}\right)^{\beta}\right] \\
\log \left(\eta_{i j}\right)=\mu_{i j}=\mathbf{x}_{i j}^{T} \gamma+\rho_{i}+\omega_{i j} \\
f_{2}\left(\rho_{i}\right)=\frac{1}{\sqrt{2 \pi \sigma_{\rho}^{2}}} e^{-\frac{\rho_{i}^{2}}{2 \sigma_{\rho}^{2}}} \\
f_{3}\left(\omega_{i j}\right)=\frac{1}{\sqrt{2 \pi \sigma_{\omega}^{2}}} e^{-\frac{\omega_{i j}^{2}}{2 \sigma_{\omega}^{2}}}
\end{gathered}
$$

where $\rho_{i}$ and $\omega_{i j}$ are all independent.

The conditional likelihood for test stand $j$ in block $i$ given the test stand and block effects is 


$$
L_{i j}\left(\boldsymbol{\gamma}, \beta, \sigma_{\rho}, \sigma_{\omega} \mid \rho_{i}, \omega_{i j}\right)=\prod_{k(i j)=1}^{n} g\left(t_{k(i j)} \mid \rho_{i}, \omega_{i j}\right)
$$

where $g\left(t_{k(i j)} \mid \rho_{j}, \omega_{i j}\right)=\left[f_{1}\left(t_{k(i j)} \mid \rho_{i}, \omega_{i j}\right)\right]^{\delta_{k(i j)}}\left[1-F_{1}\left(t_{k(i j)} \mid \rho_{i}, \omega_{i j}\right)\right]^{1-\delta_{k(i j)}}$. Furthermore, $\delta_{k(i j)}=$ 1 if the observation represents a failure and is 0 otherwise.

Then the conditional likelihood for a test stand $j$ in block $i$ given the block effect (but not the test stand effect) is

$$
L_{i j}\left(\gamma, \beta, \sigma_{\rho}, \sigma_{\omega} \mid \rho_{i}\right)=\int_{-\infty}^{\infty}\left(\prod_{k(i j)=1}^{n} g\left(t_{k(i j)} \mid \rho_{i}, \omega_{i j}\right)\right) f_{3}\left(\omega_{i j}\right) d \omega_{i j}
$$

This leads to a conditional likelihood for block $i$ of

$$
L_{i}\left(\boldsymbol{\gamma}, \beta, \sigma_{\rho}, \sigma_{\omega} \mid \rho_{i}\right)=\prod_{j=1}^{m}\left\{\int_{-\infty}^{\infty}\left(\prod_{k(i j)=1}^{n} g\left(t_{k(i j)} \mid \rho_{i}, \omega_{i j}\right)\right) f_{3}\left(\omega_{i j}\right) d \omega_{i j}\right\} .
$$

Thus, the unconditional likelihood for block $i$ is

$$
L_{i}\left(\boldsymbol{\gamma}, \beta, \sigma_{\rho}, \sigma_{\omega}\right)=\int_{-\infty}^{\infty}\left[\prod_{j=1}^{m}\left\{\int_{-\infty}^{\infty}\left(\prod_{k(i j)=1}^{n} g\left(t_{k(i j)} \mid \rho_{i}, \omega_{i j}\right)\right) f_{3}\left(\omega_{i j}\right) d \omega_{i j}\right\}\right] f_{2}\left(\rho_{j}\right) d \rho_{j}
$$

Therefore, the total joint likelihood is 
$L\left(\boldsymbol{\gamma}, \beta, \sigma_{\rho}, \sigma_{\omega} \mid D a t a\right)=\prod_{i=1}^{l}\left\{\int_{-\infty}^{\infty}\left[\prod_{j=1}^{m}\left\{\int_{-\infty}^{\infty}\left(\prod_{k(i j)=1}^{n} g\left(t_{k(i j)} \mid \rho_{i}, \omega_{i j}\right)\right) f_{3}\left(\omega_{i j}\right) d \omega_{i j}\right\}\right] f_{2}\left(\rho_{i}\right) d \rho_{i}\right\}$.

\subsection{Gauss-Hermite Quadrature}

In order to find maximum likehood estimates using the likelihood in equation 5.5, the integration must be performed. However, the integrals in equation 5.5 are intractable. Thus, a numerical method must be used. In order to perform the desired inferences the observed information matrix must be obtained. Therefore, the numerical method used to perform the integration must result in a closed-form solution. One such technique is Gauss-Hermite quadrature. Pinheiro and Bates (1995), Pinheiro and Chao (2006), and Raudenbush et al. (2000) discuss the implementation of Gauss-Hermite quadrature for multiple random effects.

Gauss-Hermite quadrature is a numerical integration technique for solving the integral $\int_{-\infty}^{\infty} e^{-x^{2}} f(x) d x$. Using substitution, Gauss-Hermite quadrature can be used to integrate out normal random effects in the likelihood. First, consider integrating out the test stand effect:

$$
\int_{-\infty}^{\infty}\left(\prod_{k(i j)=1}^{n} g\left(t_{k(i j)} \mid \rho_{i}, \omega_{i j}\right)\right) f_{3}\left(\omega_{i j}\right) d \omega_{i j}
$$


First, insert $f_{3}\left(\omega_{i j}\right)$ giving

$$
\int_{-\infty}^{\infty}\left(\prod_{k(i j)=1}^{n} g\left(t_{k(i j)} \mid \rho_{i}, \omega_{i j}\right)\right) \frac{1}{\sqrt{2 \pi \sigma_{\omega}^{2}}} e^{-\frac{\omega_{i j}^{2}}{2 \sigma_{\omega}^{2}}} d \omega_{i j} .
$$

The exponent must be of the form $e^{-x^{2}}$, so let $\omega_{i j}=\sqrt{2} \sigma_{\omega} o_{i j}$. Using substitution gives

$$
\int_{-\infty}^{\infty}\left(\prod_{k(i j)=1}^{n} g\left(t_{k(i j)} \mid \rho_{i}, \sqrt{2} \sigma_{\omega} o_{i j}\right)\right) \frac{1}{\sqrt{\pi}} e^{-o_{i j}^{2}} d o_{i j} .
$$

Using Gauss-Hermite quadrature gives the following approximation of this integral:

$$
\frac{1}{\sqrt{\pi}} \sum_{k 1=1}^{n_{k 1}} w_{1, k 1}\left(\prod_{k(i j)}^{n} g\left(t_{k(i j)} \mid \rho_{i}, \sqrt{2} \sigma_{\omega} q_{1, k 1}\right)\right)
$$

where $n_{k 1}$ is the number of quadrature points, $q_{1, k 1}$ are the evaluation points and $w_{1, k 1}$ are the weights. The evaluation points are the roots of the Hermite polynomial of degree $n_{k 1}$, $H_{n_{k 1}}\left(q_{1}\right)$, and the weights are

$$
w_{1, k 1}=\frac{2^{n_{k 1}-1} n_{k 1} ! \sqrt{\pi}}{n_{k 1}^{2}\left[H_{n_{k 1}-1}\left(q_{1, k 1}\right)\right]^{2}} .
$$

Thus, integrating out the test stand effect gives the approximate likelihood 
$L\left(\boldsymbol{\gamma}, \beta, \sigma_{\rho}, \sigma_{\omega} \mid\right.$ Data $) \approx \prod_{i=1}^{l}\left\{\pi^{-m / 2} \int_{-\infty}^{\infty} f_{2}\left(\rho_{i}\right)\left[\prod_{j=1}^{m}\left\{\sum_{k 1=1}^{n_{k 1}} w_{1, k 1}\left(\prod_{k(i j)=1}^{n} g\left(t_{k(i j)} \mid \rho_{i}, \sqrt{2} \sigma_{\omega} q_{1, k 1}\right)\right)\right\}\right] d \rho_{i}\right\}$.

The next step involves integrating out the random block effect. This integration is performed by using a second iteration of Gauss-Hermite quadrature. The integral to be evaluated is

$$
\int_{-\infty}^{\infty} f_{2}\left(\rho_{i}\right)\left[\prod_{j=1}^{m}\left\{\sum_{k 1=1}^{n_{k 1}} w_{1, k 1}\left(\prod_{k(i j)=1}^{n} g\left(t_{k(i j)} \mid \rho_{i}, \sqrt{2} \sigma_{\omega} q_{1, k 1}\right)\right)\right\}\right] d \rho_{i}
$$

Entering $f_{2}\left(\rho_{i}\right)$ gives

$$
\int_{-\infty}^{\infty}\left[\prod_{j=1}^{m}\left\{\sum_{k 1=1}^{n_{k 1}} w_{1, k 1}\left(\prod_{k(i j)=1}^{n} g\left(t_{k(i j)} \mid \rho_{i}, \sqrt{2} \sigma_{\omega} q_{1, k 1}\right)\right)\right\}\right] \frac{1}{\sqrt{2 \pi \sigma_{\rho}^{2}}} e^{-\frac{\rho_{i}^{2}}{2 \sigma_{\rho}^{2}}} d \rho_{i}
$$

Let $\rho_{i}=\sqrt{2} \sigma_{\rho} r_{i}$. Using substitution yields

$$
\int_{-\infty}^{\infty}\left[\prod_{j=1}^{m}\left\{\sum_{k 1=1}^{n_{k 1}} w_{1, k 1}\left(\prod_{k(i j)=1}^{n} g\left(t_{k(i j)} \mid \sqrt{2} \sigma_{\rho} r_{i}, \sqrt{2} \sigma_{\omega} q_{1, k 1}\right)\right)\right\}\right] \frac{1}{\sqrt{\pi}} e^{-r_{i}^{2}} d r_{i}
$$

Applying Gauss-Hermite quadrature gives

$$
\frac{1}{\sqrt{\pi}} \sum_{k 2=1}^{n_{k 2}}\left[w_{2, k 2} \prod_{j=1}^{m}\left\{\sum_{k 1=1}^{n_{k 1}} w_{1, k 1}\left(\prod_{k(i j)=1}^{n} g\left(t_{k(i j)} \mid \sqrt{2} \sigma_{\rho} q_{2, k 2}, \sqrt{2} \sigma_{\omega} q_{1, k 1}\right)\right)\right\}\right]
$$


where $n_{k 2}$ is the number of quadrature points, $q_{2, k 2}$ are the evaluation points and $w_{2, k 2}$ are the weights. The evaluation points are the roots of the Hermite polynomial of degree $n_{k 2}$, $H_{n_{k 2}}\left(q_{2}\right)$, and the weights are

$$
w_{2, k 2}=\frac{2^{n_{k 2}-1} n_{k 2} ! \sqrt{\pi}}{n_{k 2}^{2}\left[H_{n_{k 2}-1}\left(q_{2, k 2}\right)\right]^{2}} .
$$

Therefore, a closed form approximate likelihood is

$$
\begin{aligned}
L\left(\boldsymbol{\gamma}, \beta, \sigma_{\rho}, \sigma_{\omega} \mid \text { Data }\right) \approx \prod_{i=1}^{l}\left\{\pi^{-(m+1) / 2}\right. & \\
& \times \sum_{k 2=1}^{n_{k 2}}\left[w_{2, k 2} \prod_{j=1}^{m}\left\{\sum_{k 1=1}^{n_{k 1}} w_{1, k 1}\left(\prod_{k(i j)=1}^{n} g\left(t_{k(i j)} \mid \sqrt{2} \sigma_{\rho} q_{2, k 2}, \sqrt{2} \sigma_{\omega} q_{1, k 1}\right)\right)\right\}\right] .
\end{aligned}
$$

Thus, an approximate log-likelihood is

$$
\begin{aligned}
\mathcal{L}\left(\boldsymbol{\gamma}, \beta, \sigma_{\rho}, \sigma_{\omega} \mid \text { Data }\right) \approx \sum_{i=1}^{l} & \log \left\{\pi^{-(m+1) / 2}\right. \\
& \times \sum_{k 2=1}^{n_{k 2}}\left[w_{2, k 2} \prod_{j=1}^{m}\left\{\sum_{k 1=1}^{n_{k 1}} w_{1, k 1}\left(\prod_{k(i j)=1}^{n} g\left(t_{k(i j)} \mid \sqrt{2} \sigma_{\rho} q_{2, k 2}, \sqrt{2} \sigma_{\omega} q_{1, k 1}\right)\right)\right\}\right] .
\end{aligned}
$$


This closed form approximate log-likelihood allows the estimation of the variance-covariance matrix for parameter estimates.

The asymptotic variance-covariance matrix is

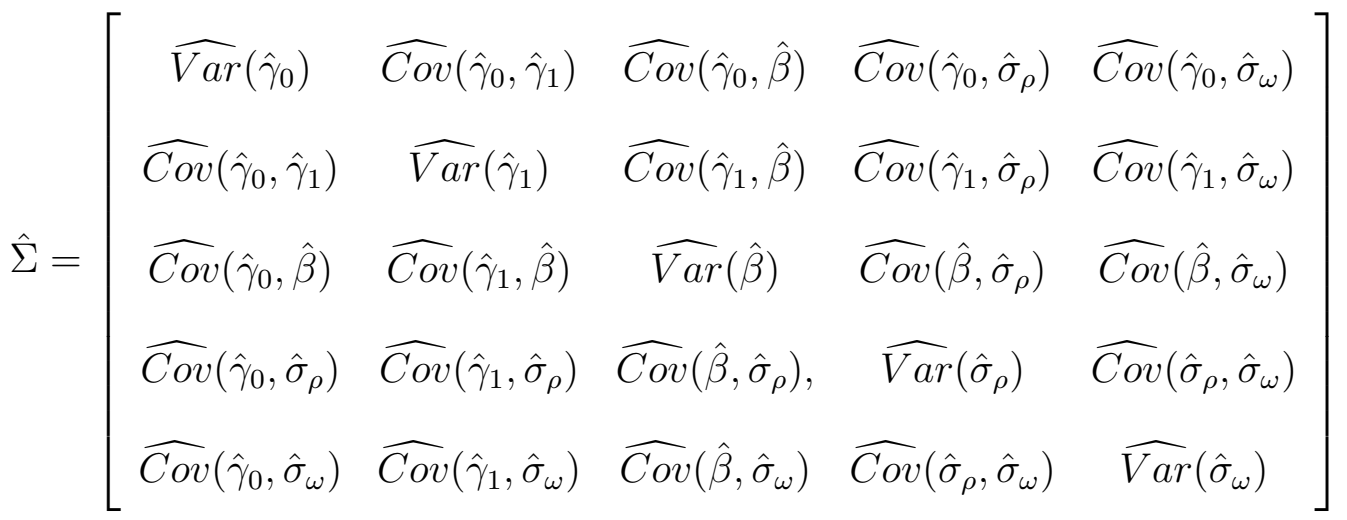

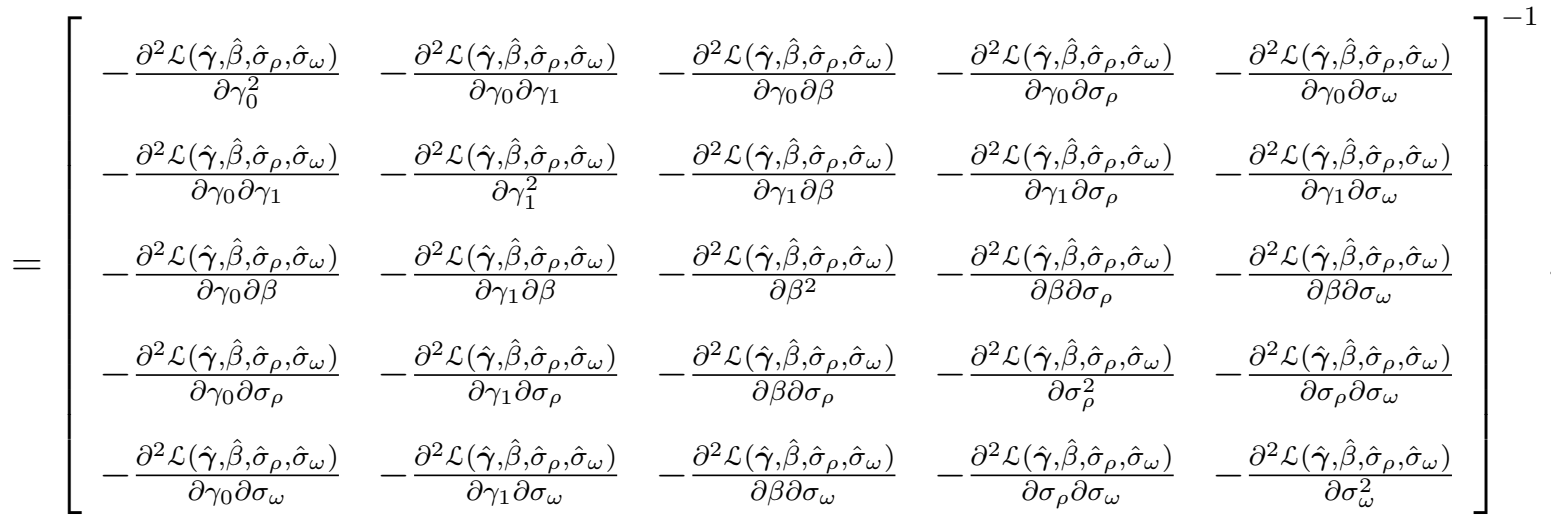

Appendix B contains the formulas for these second order partial derivatives. 


\subsection{Inference}

\subsubsection{Wald Inferences}

Wald confidence intervals can be obtained in a manner analogous to those in Chapter 2. Let $\boldsymbol{\theta}^{T}=\left(\gamma_{0}, \gamma_{1}, \beta, \sigma_{\rho}, \sigma_{\omega}\right)$. Then tests and confidence intervals may be based on $Z_{\hat{\theta}}=[\hat{\theta}-\theta] / \widehat{s e}_{\hat{\theta}}$ having an approximate standard normal distribution.

A $100(1-\alpha) \%$ confidence interval for $\theta$ is

$$
\hat{\theta} \pm z_{1-\alpha / 2} \widehat{s e}_{\hat{\theta}}
$$

where $\widehat{s e}_{\hat{\theta}}=\sqrt{\widehat{\operatorname{Var}}(\hat{\theta})}$ is the square root of the appropriate diagonal element in the variancecovariance matrix.

Tests may be performed in the usual manner. The test statistic is

$$
z_{o b s}=\frac{\hat{\theta}-\theta_{0}}{\widehat{s e}_{\hat{\theta}}}
$$

where $\theta_{0}$ is the assumed value of $\theta$.

Since $\beta, \sigma_{\rho}$ and $\sigma_{\omega}$ are greater than 0 , using a $\log$ transformation to compute their confidence intervals will ensure that the endpoints of the intervals are greater than 0 . These intervals are based on the distribution of $\frac{\log (\hat{\theta})-\log (\theta)}{\hat{s} e_{\log (\hat{\theta})}}$ being approximately $N(0,1)$. In this case, the 
interval

$$
\left(\frac{\hat{\theta}}{w}, \hat{\theta} \times w\right)
$$

produces a $100(1-\alpha) \%$ confidence interval for $\theta$, where $w=\exp \left[z_{1-\alpha / 2} \widehat{s e} \hat{\theta} / \hat{\theta}\right]$ and $\widehat{s e}_{\hat{\theta}}=$ $\sqrt{\widehat{\operatorname{Var}}(\hat{\theta})}$.

Certain functions of parameters, such as the $\log$-scale parameter $\mu$, are also of interest. The log-scale parameter is estimated by

$$
\hat{\mu}=\hat{\gamma}_{0}+\hat{\gamma}_{1} x
$$

A $100(1-\alpha) \%$ confidence interval for $\mu$ is

$$
\hat{\mu} \pm z_{1-\alpha / 2} \widehat{s e}_{\hat{\mu}}
$$

where $\widehat{\operatorname{se}} \hat{\mu}_{\hat{\mu}}=\sqrt{\widehat{\operatorname{Var}}\left(\hat{\gamma}_{0}\right)+2 x \widehat{\operatorname{Cov}}\left(\hat{\gamma}_{0}, \hat{\gamma}_{1}\right)+x^{2} \widehat{\operatorname{Var}}\left(\hat{\gamma}_{1}\right)}$. Wald tests for $\mu$ are performed in the usual manner with the test statistic

$$
z_{o b s}=\frac{\hat{\mu}-\mu_{0}}{\widehat{s e}_{\hat{\mu}}}
$$

Another function of interest is the $p^{t h}$ percentile. The $p^{t h}$ percentile, $t_{p}$, is estimated by 


$$
\hat{t}_{p}=\exp \left[\hat{\mu}+\frac{\Phi^{-1}(p)}{\hat{\beta}}\right]
$$

For $t_{p}$ the interval

$$
\left(\frac{\hat{t}_{p}}{w}, \hat{t}_{p} \times w\right)
$$

gives a $100(1-\alpha) \%$ confidence interval, where $w=\exp \left[z_{1-\alpha / 2} \widehat{s}_{\hat{t}_{p}} / \hat{t}_{p}\right]$ and

$$
\widehat{s e}_{\hat{t}_{p}}=\hat{t}_{p}\left\{\widehat{\operatorname{Var}}(\hat{\mu})-2 \frac{\Phi^{-1}(p)}{\hat{\beta}^{2}} \widehat{\operatorname{Cov}}(\hat{\mu}, \hat{\beta})+\left(\frac{\Phi^{-1}(p)}{\hat{\beta}^{2}}\right)^{2} \widehat{\operatorname{Var}}(\hat{\beta})\right\}^{1 / 2} .
$$

This interval is based on the distribution of $\frac{\log \left(\hat{t}_{p}\right)-\log \left(t_{p}\right)}{\tilde{s}_{\log \left(t_{p}\right)}}$ being approximately $N(0,1)$. Recall that

$$
\widehat{\operatorname{Var}}(\hat{\mu})=\widehat{\operatorname{Var}}\left(\hat{\gamma}_{0}\right)+2 x \widehat{\operatorname{Cov}}\left(\hat{\gamma}_{0}, \hat{\gamma}_{1}\right)+x^{2} \widehat{\operatorname{Var}}\left(\hat{\gamma}_{1}\right)
$$

and

$$
\widehat{\operatorname{Cov}}(\hat{\mu}, \hat{\beta})=\widehat{\operatorname{Cov}}\left(\hat{\gamma}_{0}, \hat{\beta}\right)+x \widehat{\operatorname{Cov}}\left(\hat{\gamma}_{1}, \hat{\beta}\right)
$$

The Wald test for $t_{p}$ based on the log transformation has a test statistic of 


$$
z_{o b s}=\frac{\log \left(\hat{t}_{p}\right)-\log \left(t_{p_{0}}\right)}{\widehat{s e}_{\log \left(\hat{t}_{p}\right)}}
$$

where

$$
\widehat{\operatorname{se}}_{\log \left(\hat{t}_{p}\right)}=\left\{\widehat{\operatorname{Var}}(\hat{\mu})-2 \frac{\Phi^{-1}(p)}{\hat{\beta}^{2}} \widehat{\operatorname{Cov}}(\hat{\mu}, \hat{\beta})+\left(\frac{\Phi^{-1}(p)}{\hat{\beta}^{2}}\right)^{2} \widehat{\operatorname{Var}}(\hat{\beta})\right\}^{1 / 2} .
$$

\subsubsection{Inference Based on the Likelihood}

Inferences based on the likelihood may be performed in a manner analogous to those in Chapter 2. For inferences about $\boldsymbol{\theta}_{1}$, the parameter space can be partitioned as $\boldsymbol{\theta}=\left(\boldsymbol{\theta}_{1}, \boldsymbol{\theta}_{2}\right)$ where $p$ is the length of $\boldsymbol{\theta}_{1}$. The profile likelihood for $\boldsymbol{\theta}_{1}$ is

$$
R\left(\boldsymbol{\theta}_{1}\right)=\max _{\boldsymbol{\theta}_{2}}\left[\frac{L\left(\boldsymbol{\theta}_{1}, \boldsymbol{\theta}_{2}\right)}{L(\hat{\boldsymbol{\theta}})}\right] .
$$

Then a $100(1-\alpha) \%$ confidence region for $\boldsymbol{\theta}_{1}$ is the region where

$$
R\left(\boldsymbol{\theta}_{1}\right)>\exp \left[-\chi_{1-\alpha, p}^{2} / 2\right] .
$$

Next consider testing $H_{0}: \boldsymbol{\theta}_{1}=\boldsymbol{\theta}_{10}$. The the likelihood ratio test statistic is 


$$
L L R\left(\boldsymbol{\theta}_{1}\right)=-2 \log \left[R\left(\boldsymbol{\theta}_{10}\right)\right]=-2 \log \left(\frac{L(\tilde{\boldsymbol{\theta}})}{L(\hat{\boldsymbol{\theta}})}\right)=-2 \mathcal{L}(\tilde{\boldsymbol{\theta}})-(-2 \mathcal{L}(\hat{\boldsymbol{\theta}}))
$$

where $\tilde{\boldsymbol{\theta}}=\left(\boldsymbol{\theta}_{10}, \tilde{\boldsymbol{\theta}}_{2}\left(\boldsymbol{\theta}_{10}\right)\right)$ and $\tilde{\boldsymbol{\theta}}_{2}\left(\boldsymbol{\theta}_{10}\right)=\max _{\boldsymbol{\theta}_{2}} L\left(\boldsymbol{\theta}_{10}, \boldsymbol{\theta}_{2}\right)$. The null hypothesis is rejected when $\operatorname{LLR}\left(\boldsymbol{\theta}_{1}\right)>\chi_{1-\alpha, p}^{2}$.

\section{Testing Fixed Effects}

Consider the log-scale parameter, which can be reparameterized as

$$
\boldsymbol{\mu}=\log (\boldsymbol{\eta})=\mathbf{X}_{1} \boldsymbol{\gamma}_{1}+\mathbf{X}_{2} \boldsymbol{\gamma}_{2}+\mathbf{Z} \boldsymbol{\rho}+\boldsymbol{\omega}
$$

where $\gamma_{2}=\gamma_{2}$. The hypotheses for the likelihood ratio test are $H_{0}: \gamma_{2}=0$ versus $H_{A}: \gamma_{2} \neq$ 0. The log-likelihoods may be obtained using Equation 5.18. The null hypothesis is rejected if $\operatorname{LLR}\left(\gamma_{2}\right)>\chi_{1-\alpha, 1}^{2}$.

\section{Testing the Block Effect}

Although tests for random effects are included, they should be used with caution. The blocks and subsamples are introduced through the experimental protocol and are restrictions on randomization. Thus, even insignificant block and subsampling effects should not be removed from the model.

Consider the log-scale parameter, 


$$
\boldsymbol{\mu}=\log (\boldsymbol{\eta})=\mathbf{X} \boldsymbol{\gamma}+\mathbf{Z} \boldsymbol{\rho}+\boldsymbol{\omega}
$$

The hypotheses for the likelihood ratio test are $H_{0}: \sigma_{\rho}=0$ versus $H_{A}: \sigma_{\rho}>0$. The quantity $\mathcal{L}(\hat{\boldsymbol{\theta}})$ is obtained using Equation 5.18; whereas $\mathcal{L}(\tilde{\boldsymbol{\theta}})$ is obtained using

$$
\mathcal{L}\left(\boldsymbol{\gamma}, \beta, \sigma_{\omega} \mid \text { Data }\right) \approx \sum_{i=1}^{l} \sum_{j=1}^{m} \log \left(\frac{1}{\sqrt{\pi}} \sum_{k 1=1}^{n_{k 1}} w_{k 1}\left[\prod_{k(i j)=1}^{n} g\left(t_{k(i j)} \mid \sqrt{2} \sigma_{\omega} q_{k 1}\right)\right]\right),
$$

where $n_{k 1}$ is the number of quadrature points, $w_{k 1}$ are the weights and $q_{k 1}$ are the evaluation points as in Chapter 2. The null hypothesis is rejected if $L L R\left(\sigma_{\rho}\right)>\chi_{1-\alpha, 1}^{2}$.

\section{Testing the Test Stand Effect}

The hypotheses for the likelihood ratio test are $H_{0}: \sigma_{\omega}=0$ versus $H_{A}: \sigma_{\omega}>0$. The quantity $\mathcal{L}(\hat{\boldsymbol{\theta}})$ is obtained using Equation 5.18; whereas $\mathcal{L}(\tilde{\boldsymbol{\theta}})$ is obtained using

$$
\mathcal{L}\left(\boldsymbol{\gamma}, \beta, \sigma_{\rho} \mid \text { Data }\right) \approx \sum_{i=1}^{l} \log \left(\frac{1}{\sqrt{\pi}} \sum_{k 2=1}^{n_{k 2}} w_{k 2}\left[\prod_{j=1}^{m} \prod_{k(i j)=1}^{n} g\left(t_{k(i j)} \mid \sqrt{2} \sigma_{\rho} q_{k 2}\right)\right]\right)
$$

where $n_{k 2}$ is the number of quadrature points, $w_{k 2}$ are the weights and $q_{k 2}$ are the evaluation points. The null hypothesis is rejected if $L L R\left(\sigma_{\omega}\right)>\chi_{1-\alpha, 1}^{2}$. 


\subsection{An Illustrative Example}

Recall the battery life example from Chapter 4 where engineers want to study the effect of temperature on battery life. The batteries come from three batches, and for each temperature-batch combination the researchers place eight batteries in a chamber with one of three temperatures. Appendix $\mathrm{C}$ contains the $\mathrm{R}$ and SAS codes for the rigorous NLMM Wald analysis. Table 5.1 gives the estimates and standard errors for the parameters using Proc NLMIXED in SAS.

Table 5.1: NLMM Analysis Results

\begin{tabular}{|c|c|c|c|c|}
\hline Parameter & Estimate & Standard Error & P-Value & $95 \%$ CI \\
\hline$\gamma_{0}$ & 5.4776 & 0.1350 & $<0.0001$ & $(5.2130,5.7422)$ \\
$\gamma_{1}$ & -0.00684 & 0.001451 & $<0.0001$ & $(-0.0097,-0.0040)$ \\
$\beta$ & 3.1456 & 0.5676 & $<0.0001$ & $(2.2086,4.4802)^{*}$ \\
$\sigma_{\rho}$ & 0.08085 & 0.1070 & 0.4473 & $(0.0060,1.0819)^{*}$ \\
$\sigma_{\omega}$ & 0.09989 & 0.1362 & 0.4654 & $(0.0069,1.4459)^{*}$ \\
\hline
\end{tabular}

The asymptotic variance-covariance matrix of the parameter estimates is

$$
\hat{\Sigma}=\left[\begin{array}{ccccc}
0.01821 & -0.00014 & -0.2300 & 0.001542 & -0.00396 \\
-0.00014 & 2.107 \mathrm{E}-6 & -0.00001 & -0.00001 & -3.77 \mathrm{E}-6 \\
-0.2300 & -0.00001 & 0.3222 & -0.00526 & 0.03328 \\
0.001542 & -0.00001 & -0.00526 & 0.01146 & -0.00666 \\
-0.00396 & -3.77 \mathrm{E}-6 & 0.03328 & -0.00666 & 0.01856
\end{array}\right]
$$


The following table compares the results of the the traditional analysis, traditional analysis with blocks, two-stage analysis and NLMM analysis.

Table 5.2: Estimates and Standard Errors for the Traditional Analysis, Traditional Analysis with blocks, Two-Stage Analysis and NLMM Analysis

\begin{tabular}{|c|cc|cc|cc|cc|}
\hline \multirow{2}{*}{ Parameter } & \multicolumn{2}{|c|}{ Traditional Analysis } & \multicolumn{2}{|c|}{$\begin{array}{c}\text { Traditional Analysis } \\
\text { with Blocks }\end{array}$} & \multicolumn{2}{c|}{ Two-Stage Analysis } & \multicolumn{2}{c|}{ NLMM Analysis } \\
\cline { 2 - 9 } & Estimate & SE & Estimate & SE & Estimate & SE & Estimate & SE \\
\hline$\gamma_{0}$ & 5.4981 & 0.1164 & 5.6644 & 0.1355 & 5.3999 & 0.1399 & 5.4776 & 0.1350 \\
$\gamma_{1}$ & -0.0066 & 0.0014 & -0.0071 & 0.0012 & -0.0069 & 0.0017 & -0.0068 & 0.0015 \\
$\beta$ & 2.8616 & 0.4095 & 3.2148 & 0.4756 & 4.0254 & 0.6293 & 3.1456 & 0.5676 \\
$\sigma_{\rho}$ & - & - & - & - & 0.1042 & 0.0300 & 0.0809 & 0.1070 \\
$\sigma_{\omega}$ & - & - & - & - & 0.2265 & 0.0325 & 0.0999 & 0.1362 \\
\hline
\end{tabular}

Table 5.2 shows that the different methods produce different estimates of $\beta$. All four methods give similar estimates of $\gamma_{0}$ and $\gamma_{1}$. Note that the standard errors for the traditional analyses do not reflect the experimental protocol. Furthermore, the traditional analyses do not produce estimates of $\sigma_{\rho}$ and $\sigma_{\omega}$.

Another quantity of interest is the $p^{\text {th }}$ percentile. We would like to know the time by which the first $10 \%$ of items fail. Table 5.3 gives the MLEs and $95 \%$ confidence intervals for the $10^{\text {th }}$ percentile. 
Table 5.3: Estimates and Confidence intervals for $t_{0.01}$

\begin{tabular}{|c|c|c|c|c|c|}
\hline \multirow{2}{*}{\multicolumn{2}{|c|}{ Method }} & \multicolumn{4}{|c|}{ Temperature $\left({ }^{\circ} \mathrm{F}\right)$} \\
\hline & & & 15 & 70 & 125 \\
\hline \multicolumn{2}{|c|}{ Traditional Analysis } & $\begin{array}{c}\text { Estimate } \\
95 \% \text { CI }\end{array}$ & $\begin{array}{c}100.7730 \\
(78.2717,129.7430)\end{array}$ & $\begin{array}{c}70.1489 \\
(56.8582,86.5462)\end{array}$ & $\begin{array}{c}48.8312 \\
(37.5408,63.5170)\end{array}$ \\
\hline \multirow{3}{*}{$\begin{array}{c}\text { Traditional Analysis } \\
\text { with Blocks }\end{array}$} & Block 1 & $\begin{array}{l}\text { Estimate } \\
95 \% \text { CI }\end{array}$ & $\begin{array}{c}92.4307 \\
(70.2533,121.6092)\end{array}$ & $\begin{array}{c}62.6269 \\
(49.1402,79.8150)\end{array}$ & $\begin{array}{c}42.4331 \\
(32.1805,55.9522)\end{array}$ \\
\hline & Block 2 & $\begin{array}{l}\text { Estimate } \\
95 \% \text { CI }\end{array}$ & $\begin{array}{c}105.2253 \\
(81.4162,135.9971)\end{array}$ & $\begin{array}{c}71.2959 \\
(56.3568,90.1950)\end{array}$ & $\begin{array}{c}48.3069 \\
(36.4770,63.9733)\end{array}$ \\
\hline & Block 3 & $\begin{array}{l}\text { Estimate } \\
95 \% \text { CI }\end{array}$ & $\begin{array}{c}128.7998 \\
(96.9428,171.1256)\end{array}$ & $\begin{array}{c}87.2689 \\
(68.7971,110.7004)\end{array}$ & $\begin{array}{c}59.1294 \\
(45.6865,76.5278)\end{array}$ \\
\hline \multicolumn{2}{|c|}{ Two-Stage Analysis } & $\begin{array}{l}\text { Estimate } \\
95 \% \text { CI }\end{array}$ & $\begin{array}{c}114.09511 \\
-\end{array}$ & $\begin{array}{c}77.95693 \\
-\end{array}$ & $\begin{array}{c}53.26506 \\
-\end{array}$ \\
\hline \multicolumn{2}{|c|}{ NLMM Analysis } & $\begin{array}{l}\text { Estimate } \\
95 \% \text { CI }\end{array}$ & $\begin{array}{c}105.5859 \\
(79.7714,139.7540)\end{array}$ & $\begin{array}{c}72.4613 \\
(57.4333,91.4215)\end{array}$ & $\begin{array}{c}49.7286 \\
(37.5836,65.7982)\end{array}$ \\
\hline
\end{tabular}

The traditional analysis with blocks uses fixed block effects, so confidence intervals are computed for each block (or batch of material). However, manufacturers may be interested in estimating battery life for all batches, not for a particular batch. Therefore, examining confidence intervals for a particular batch may not be appropriate. Although estimates for $t_{p}$ may be calculated from the two-stage method, confidence intervals are not easily available. Notice that the rigorous NLMM analysis produces wider intervals than the traditional analysis. This reflects the incorrect modeling of the experimental unit in the traditional analysis.

\subsection{Software for the NLMM Solution}

The NLMM solution has been obtained using both SAS' Proc NLMIXED and R. Proc NLMIXED is robust to starting values and provides and easy way to obtain the MLEs and variance-covariance matrix. One drawback is that Proc NLMIXED performs inferences 
based on the $t$ distribution, but inferences on the MLEs should be based on the normal distribution. However, this problem can be easily be avoided by setting the degrees of freedom to a large number (i.e. 10,000). Littell et al. (2006) and SAS/STAT(R) 9.2 User's Guide (SAS Institute Inc. 2008) are indispensable references for programming the NLMM solution in SAS.

Using $\mathrm{R}$ allows for more flexibility, but involves much more programming. Manuel programing in $\mathrm{R}$ allows for a different number of quadrature points for integrating out the test stand and block effects (i.e. $n_{k 1}$ and $n_{k 2}$ can be different). In SAS' NLMIXED $n_{k 1}$ and $n_{k 1}$ must be the same. One disadvantage of $\mathrm{R}$ is that it is not robust to starting values in the maximum likelihood estimation. Bad starting values for the parameters can result in the algorithm for maximum likelihood estimation failing to converge. 


\section{Chapter 6}

\section{Simulation Studies}

Chapter 6 explores the properties of the NLMM method via simulation and compares this method with the two-stage method and traditional methods. This chapter includes two simulation studies. The first simulation study extends the simulation study in Chapter 4 to include the NLMM method. This study compares the performance of estimation of parameters and inference for the four methods. The second simulation study explores estimation of and inference about $t_{0.1}$, the $10^{\text {th }}$ percentile. Only the NLMM analysis and the traditional analysis are examined in the second study.

\subsection{Parameter Estimation Study}

This section compares and contrasts the NLMM analysis, two-stage analysis, traditional analysis (assuming a completely randomized design), and traditional analysis with fixed block 
effects. This simulation uses the same scenario as the study in Chapter 4. Manufacturers want to determine the effect of two operating conditions on the lifetime of a item. The operating conditions are denoted by $x$ (with two levels denoted by -1 and 1 ). The items are produced in batches and four batches are available for the experiment. The manufacturers use a randomized complete block design where each batch feeds items for two test stands (one test stand for each operating condition). The operating condition is applied to a test stand containing $n$ items and manufacturers use Type II censoring after the first $r$ failures on each test stand.

In this study failure times follow a Weibull distribution with shape parameter $\beta=5,3,1$, and 0.5 , and $\log$-scale parameter $\log \left(\eta_{i j}\right)=\mu_{i j}=\gamma_{0}+\gamma_{1} x_{i j}+\rho_{i}+\omega_{i j}$ where $\rho_{i} \sim N\left(0, \sigma_{\rho}^{2}\right)$ and $\omega_{i j} \sim N\left(0, \sigma_{\omega}^{2}\right)$ are independent. This simulation study explores three combinations of $n$ and $r: n=8$ and $r=4, n=16$ and $r=8$, and $n=16$ and $r=12$. In addition, two sets of values are examined for $\sigma_{\rho}$ and $\sigma_{\omega}: \sigma_{\rho}=0.1732$ with $\sigma_{\omega}=0.1$, and $\sigma_{\rho}=0.2449$ with $\sigma_{\omega}=0.2$. The parameter $\gamma_{0}$ remains fixed at 5 , while $\gamma_{1}$ changes based on $\sigma_{\omega}$ and the nominal power. Recall that the NLMM uses two iterations of Gauss-Hermite quadrature. Because the total number of evaluation points is the product of the number of quadrature points for each iteration, computations quickly become very time consuming. Therefore, this study uses 5 quadrature points for each iteration. Each simulation contains 1,000 replicates. 
Figure 6.1 shows the ratio of $\hat{\beta}$ to $\beta$.

Figure 6.1: Ratio of $\hat{\beta}$ to $\beta$

(a) $\sigma_{\rho}=0.1732, \sigma_{\omega}=0.1$

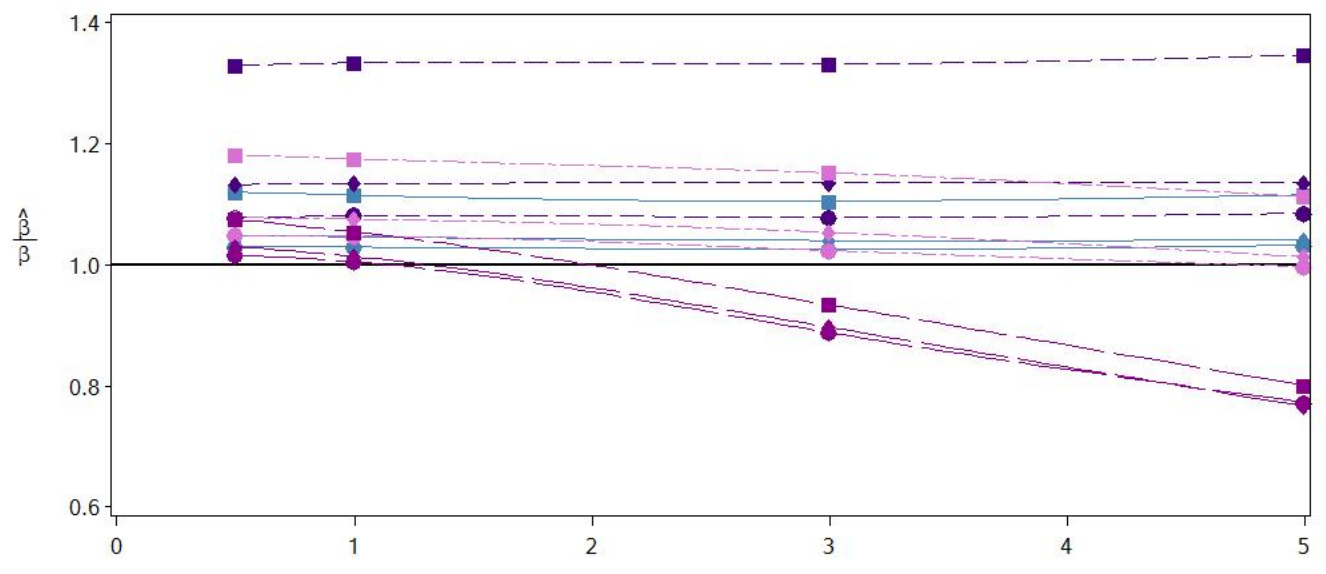

$\beta$

\begin{tabular}{|c|c|c|}
\hline $\begin{array}{l}\text { Rigorous, } n=8, r=4 \\
\text { Two-Stage, } n=8, r=4 \\
\text { Blocked Traditional, } n=8, r=4 \\
\text { Traditional, } n=8, r=4\end{array}$ & $\begin{array}{l}\text { Rigorous, } n=16, r=8 \\
\text { Two-Stage, } n=16, r=8 \\
\text { Blocked Traditional, } n=16, r=8 \\
\text { Traditional, } n=16, r=8\end{array}$ & $\begin{array}{l}\text { Rigorous, } n=16, r=12 \\
\text { Two-Stage, } n=16, r=12 \\
\text { Blocked Traditional, } n=16, r=12 \\
\text { Traditional. } n=16, r=12\end{array}$ \\
\hline
\end{tabular}

(b) $\sigma_{\rho}=0.2449, \sigma_{\omega}=0.2$

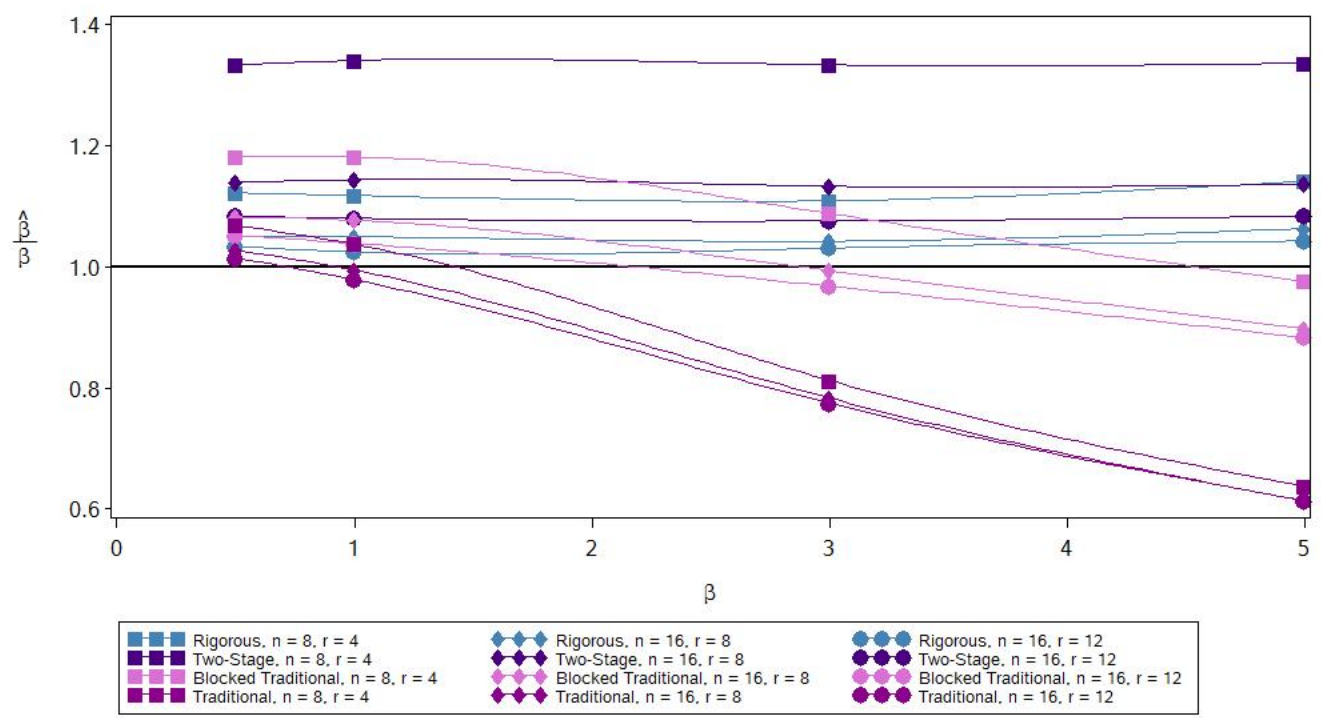


The NLMM method has the least bias in estimating $\beta$, and performs increasingly better as the number of failures on a test stand increases. The two-stage method produces biased estimates of $\beta$; however, the bias decreases as the sample size increases. Also note that the bias of the two-stage method does not depend on the value of $\beta$. Thus, it may be possible to find a bias correction factor as discussed in Chapter 2. The bias of the traditional analysis and the blocked traditional analysis depends on $\beta$ and becomes worse as $\beta$ increases. Furthermore, the performance of the traditional analysis and traditional analysis with blocks deteriorates as $\sigma_{\omega}$ increases. 
Figure 6.2 depicts the estimates of $\gamma_{0}=5$.

Figure 6.2: $\hat{\gamma}_{0}$

(a) $\gamma_{0}=5, \sigma_{\rho}=0.1732, \sigma_{\omega}=0.1$

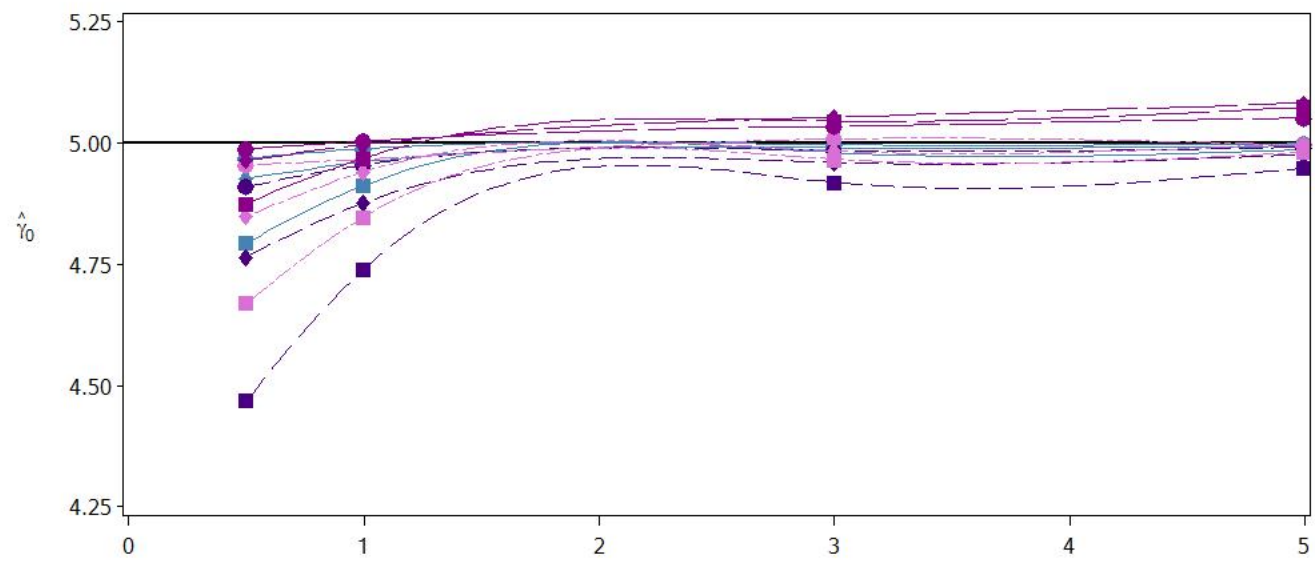

$\beta$

\begin{tabular}{|c|c|c|}
\hline $\begin{array}{l}\text { Rigorous, } n=8, r=4 \\
\text { Two-Stage, } n=8, r=4 \\
\text { Blocked Traditional, } n=8, r=4 \\
\text { Traditional, } n=8, r=4\end{array}$ & $\begin{array}{l}\text { Rigorous, } n=16, r=8 \\
\text { Two-Stage, } n=16, r=8 \\
\text { Blocked Traditional, } n=16, r=8 \\
\text { Traditional, } n=16, r=8\end{array}$ & $\begin{array}{l}\text { Rigorous, } n=16, r=12 \\
\text { Two-Stage, } n=16, r=12 \\
\text { Blocked Traditional, } n=16, r=12 \\
\text { Traditional, } n=16, r=12\end{array}$ \\
\hline
\end{tabular}

(b) $\gamma_{0}=5, \sigma_{\rho}=0.2449, \sigma_{\omega}=0.2$

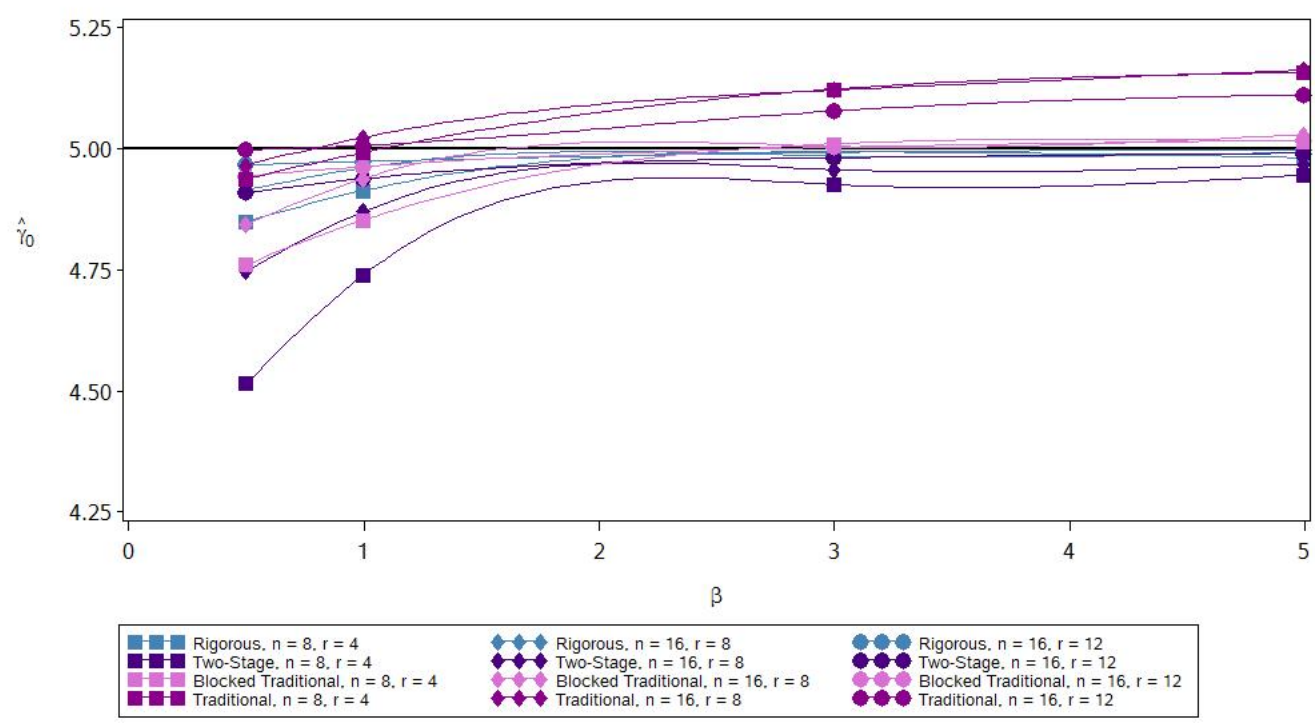


The NLMM, two-stage, and traditional analysis with fixed blocks perform similarly for both values of $\sigma_{\omega}$, while the traditional analysis becomes increasingly biased for the larger value of $\sigma_{\omega}$. Overall the NLMM performs the best in the estimation of $\gamma_{0}$, and the estimation improves for all three methods as the number of failures on a test stand increases. Figure 6.3 gives the estimates of $\gamma_{1}=0$ (i.e. the value of $\gamma_{1}$ when operating condition does not affect item life). 
Figure 6.3: $\hat{\gamma}_{1}$

(a) $\gamma_{1}=0, \sigma_{\rho}=0.1732, \sigma_{\omega}=0.1$

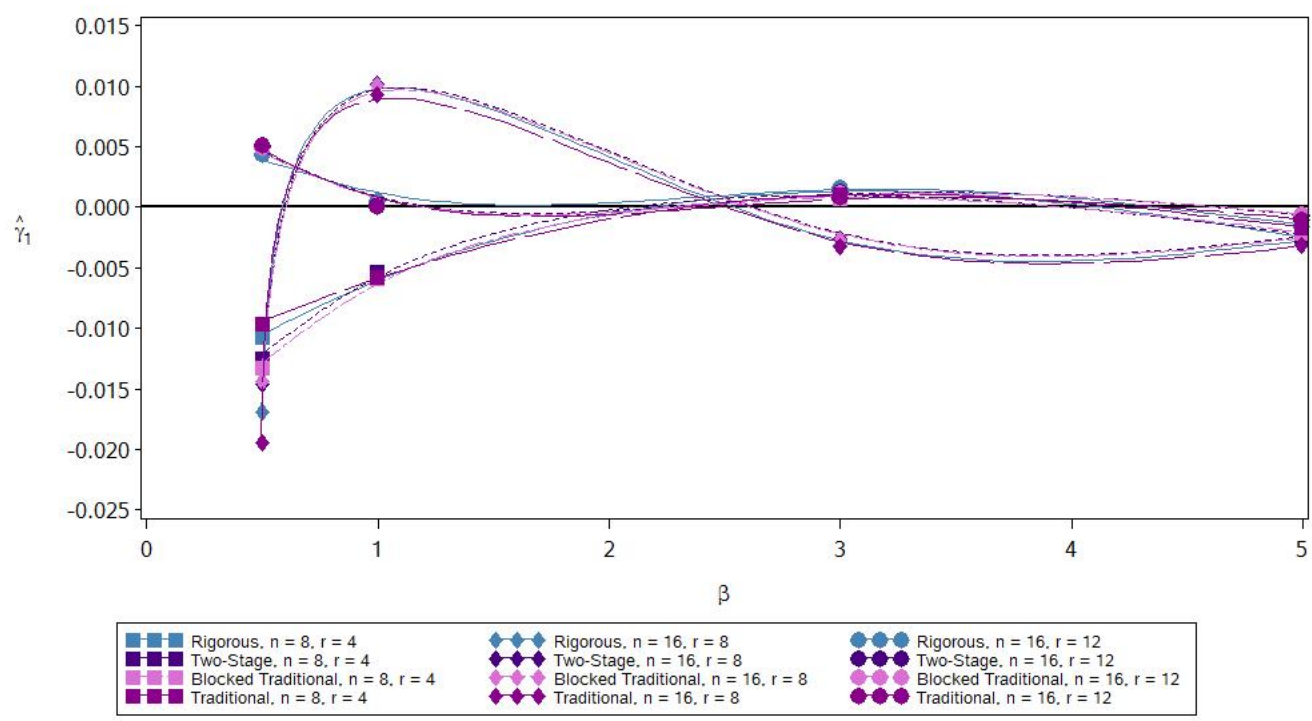

(b) $\gamma_{1}=0, \sigma_{\rho}=0.2449, \sigma_{\omega}=0.2$

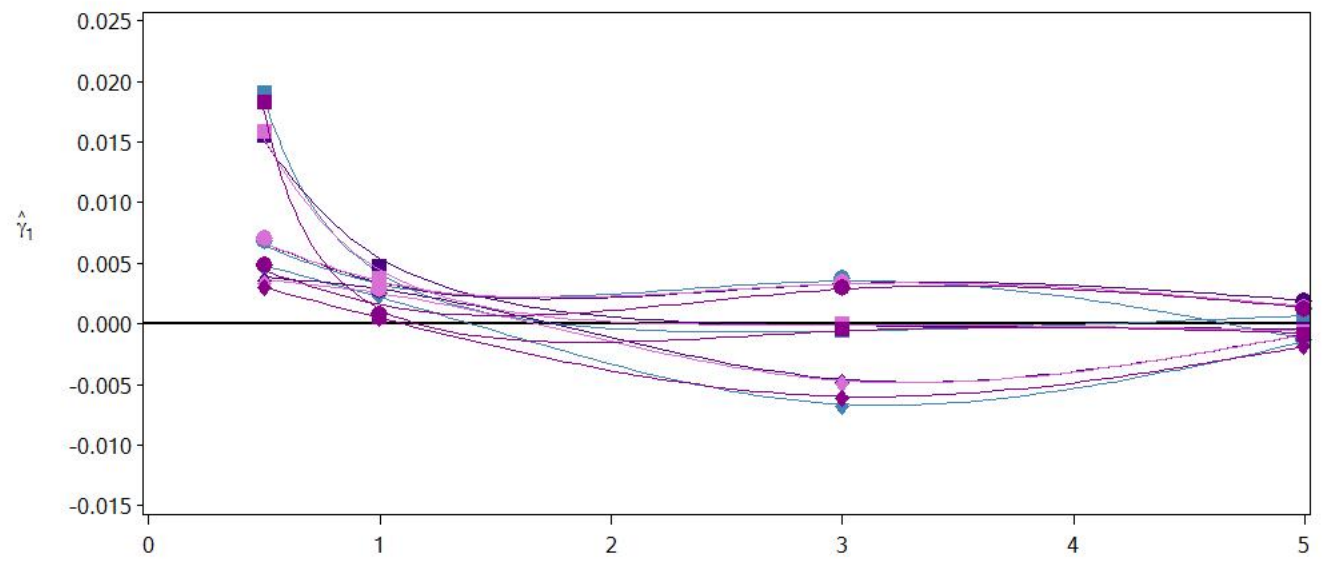

$\beta$

\begin{tabular}{|c|c|c|}
\hline $\begin{array}{l}\text { Rigorous, } n=8, r=4 \\
\text { Two-Stage. } n=8, r=4 \\
\text { Blocked Traditional. } n=8, r=4 \\
\text { Traditional. } n=8, r=4\end{array}$ & $\begin{array}{l}\text { Rigorous, } n=16, r=8 \\
\text { Two-Stage, } n=16, r=8 \\
\text { Blocked Traditional, } n=16, r=8 \\
\text { Traditional, } n=16, r=8\end{array}$ & $\begin{array}{l}\text { Rigorous, } n=16, r=12 \\
\text { Two-Stage. } n=16, r=12 \\
\text { Blocked Traditional, } n=16, r=12 \\
\text { Traditional, } n=16, r=12\end{array}$ \\
\hline
\end{tabular}


All three methods perform similarly, with sample size affecting the performance of the estimation. Figure 6.4 shows $\gamma_{1}$ calculated to have a nominal power of 0.50 under stage 2 in the two-stage method. Under the assumption that $\gamma_{1}=0$, the $P\left(F^{*}>F_{0.95,1,3, \lambda=0}=\right.$ 10.12796) $=0.05$ where $F^{*}$ is the observed $F$ statistic in stage 2 of the two-stage analysis. For this design the noncentrality parameter is $\lambda=\frac{8 \gamma_{1}^{2}}{\sigma_{\omega}^{2}}$. To find the value of $\gamma_{1}$ that will give a nominal power of 0.50 , first find the value of $\lambda$ such that $P\left(F^{*}>5.987378 \mid \lambda\right)=0.50$. Solving for $\lambda$ gives $\lambda=8.22697$. Next, substitute $\lambda$ into the equation $\lambda=\frac{8 \gamma_{1}^{2}}{\sigma_{\omega}^{2}}$. Solving for $\gamma_{1}$ we obtain $\gamma_{1}= \pm 0.10141$ and $\gamma_{1}= \pm 1.20282$ for $\sigma_{\omega}=0.1$ and $\sigma_{\omega}=0.2$ respectively. These simulations use the positive solutions of $\gamma_{1}$. Figure 6.4 illustrates the estimation of $\gamma_{1}$ when optimized for a nominal power of 0.50 . 
Figure 6.4: $\hat{\gamma}_{1}$ for Nominal Power 0.50

(a) $\gamma_{1}=0.10141, \sigma_{\rho}=0.1732$ and $\sigma_{\omega}=0.1$

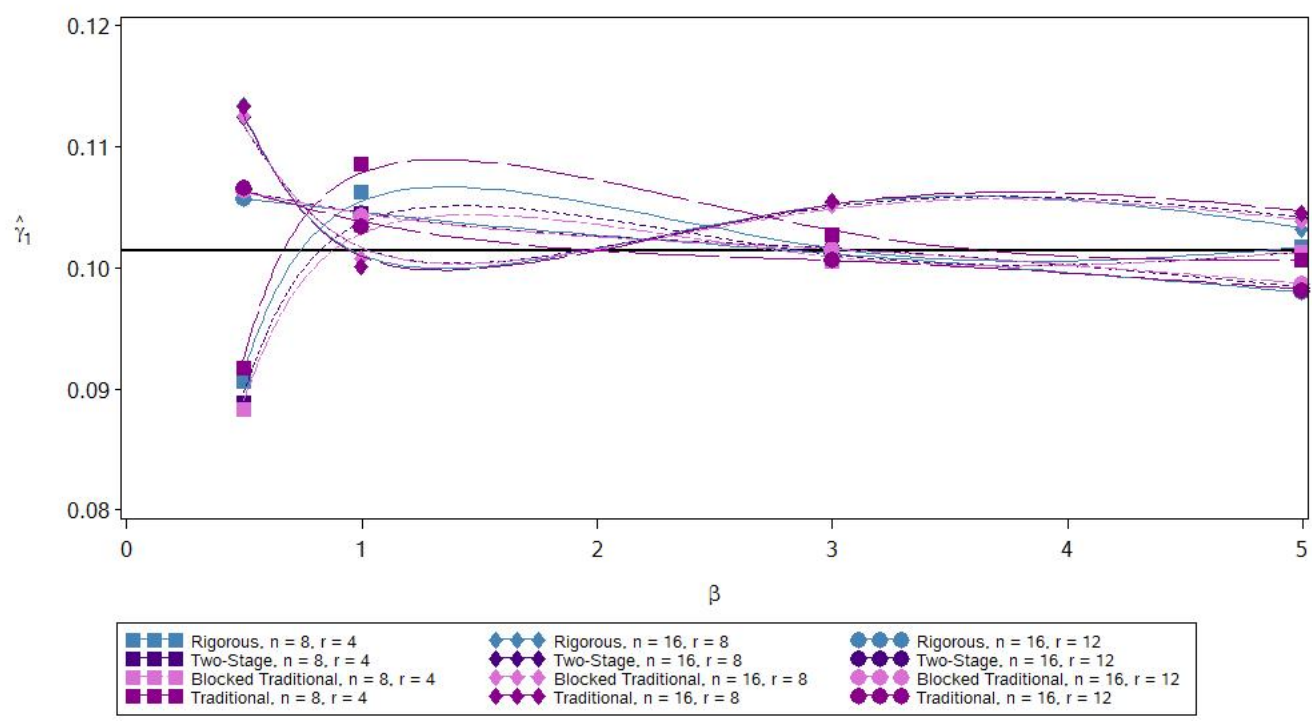

(b) $\gamma_{1}=0.20282, \sigma_{\rho}=0.2449$ and $\sigma_{\omega}=0.2$

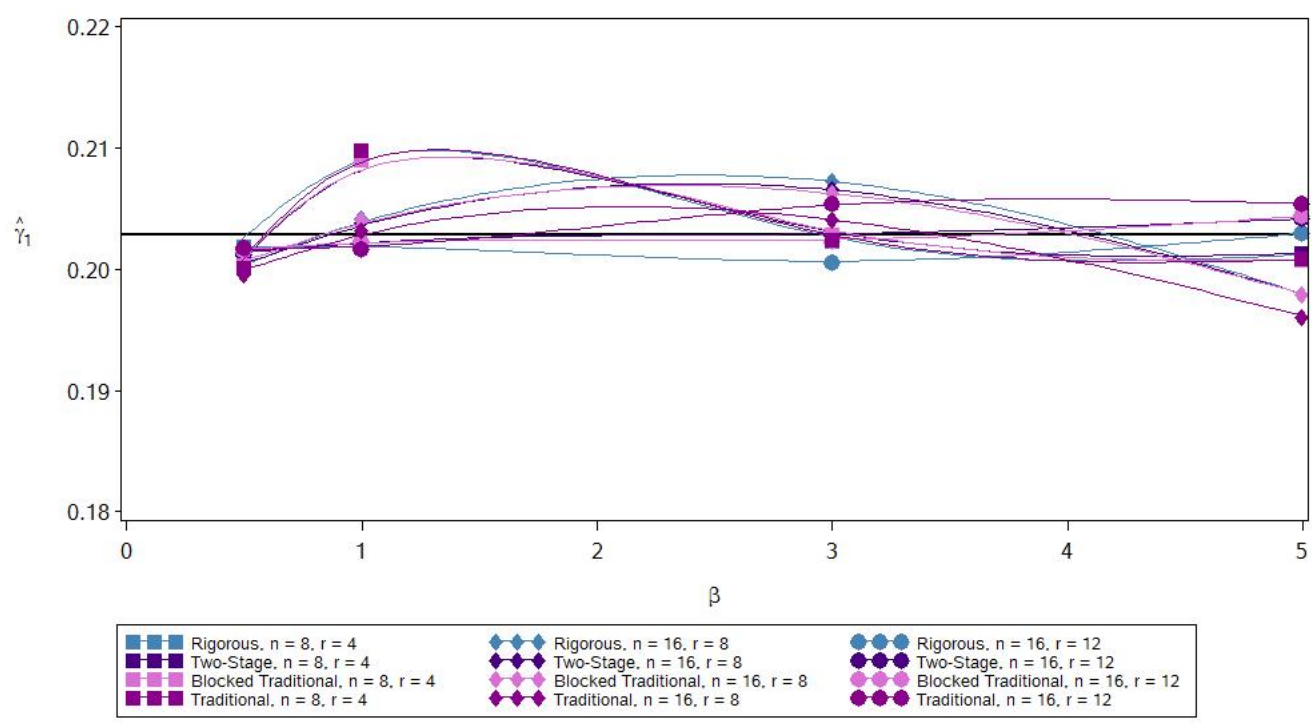


Estimation of $\gamma_{1}$ is very similar for the NLMM, two-stage and traditional analyses. Figure 6.5 shows the estimation of $\gamma_{1}$ with a nominal power of 0.80 .

Figure 6.5: $\hat{\gamma}_{1}$ for Nominal Power 0.80

(a) $\gamma_{1}=0.15047, \sigma_{\rho}=0.1732$ and $\sigma_{\omega}=0.1$
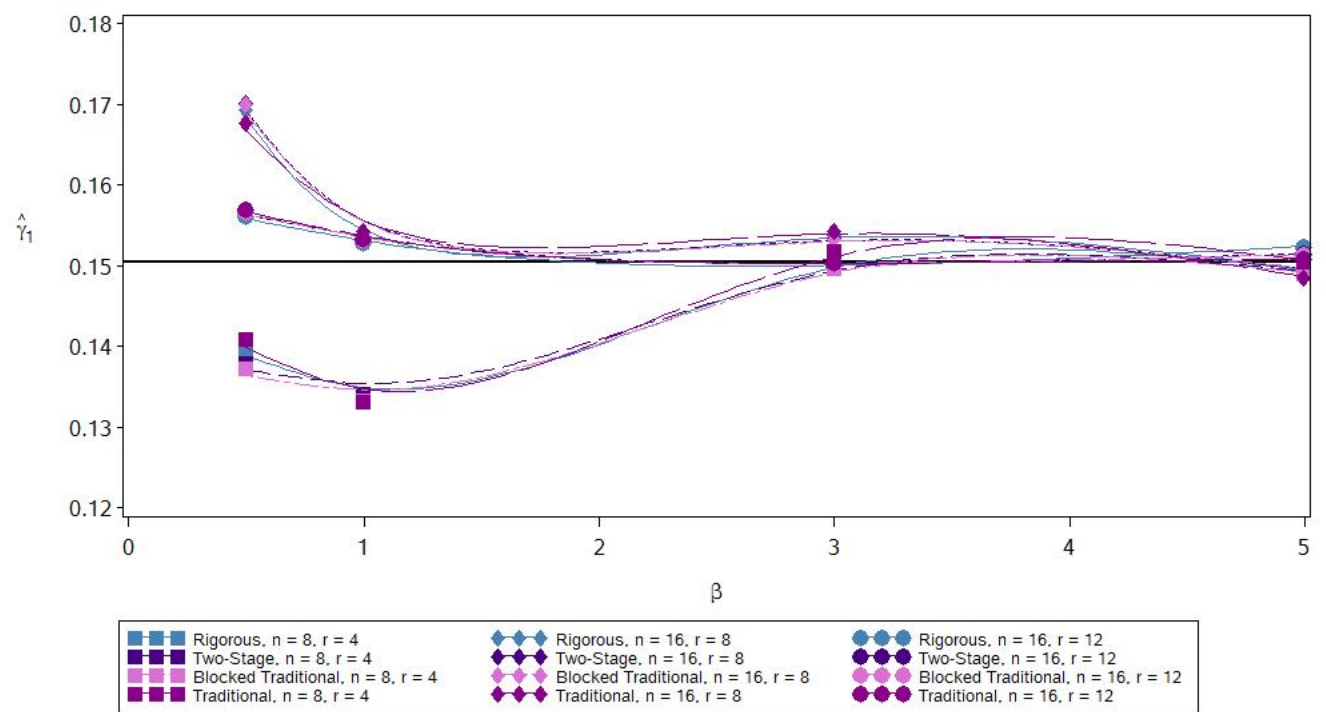

(b) $\gamma_{1}=0.30094, \sigma_{\rho}=0.2449$ and $\sigma_{\omega}=0.2$

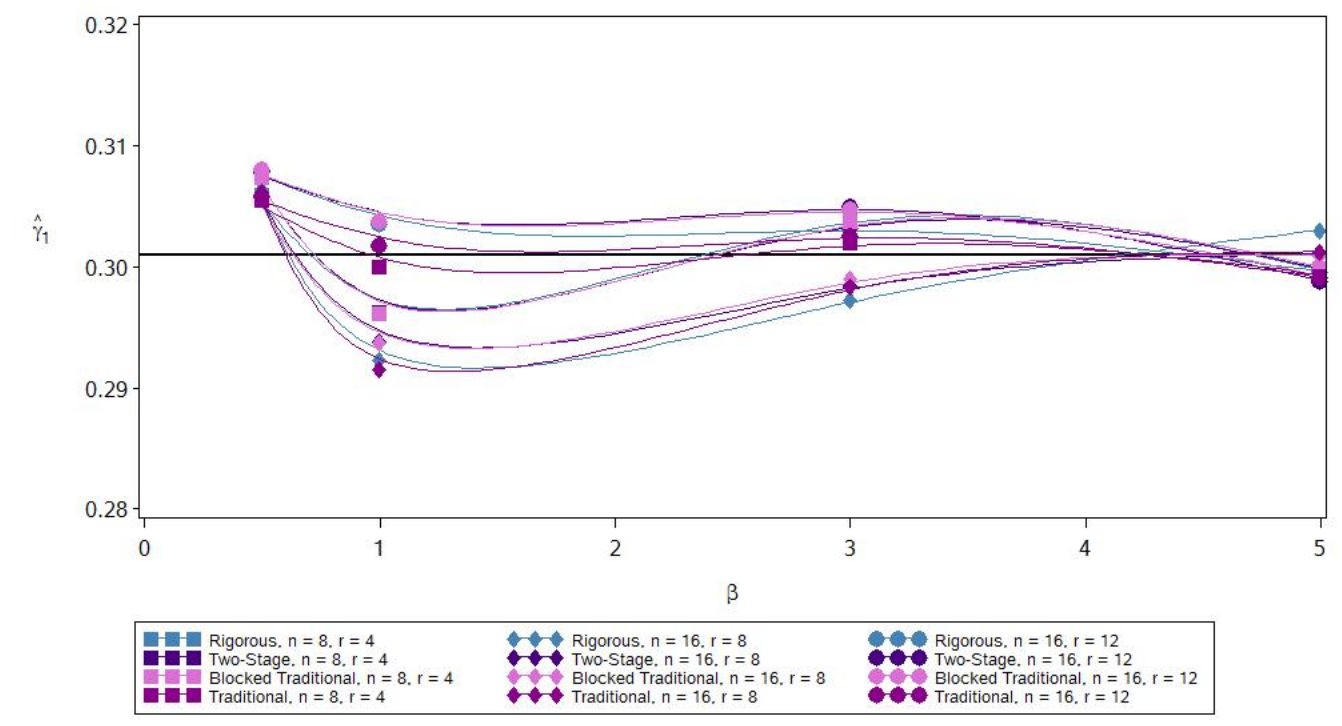


Again, estimates of $\gamma_{1}$ appear similar for all three methods. Figure 6.6 shows the estimates of $\gamma_{1}$ for a nominal power of 0.90 .

Figure 6.6: $\hat{\gamma}_{1}$ for Nominal Power 0.90

(a) $\gamma_{1}=0.17726, \sigma_{\rho}=0.1732$ and $\sigma_{\omega}=0.1$
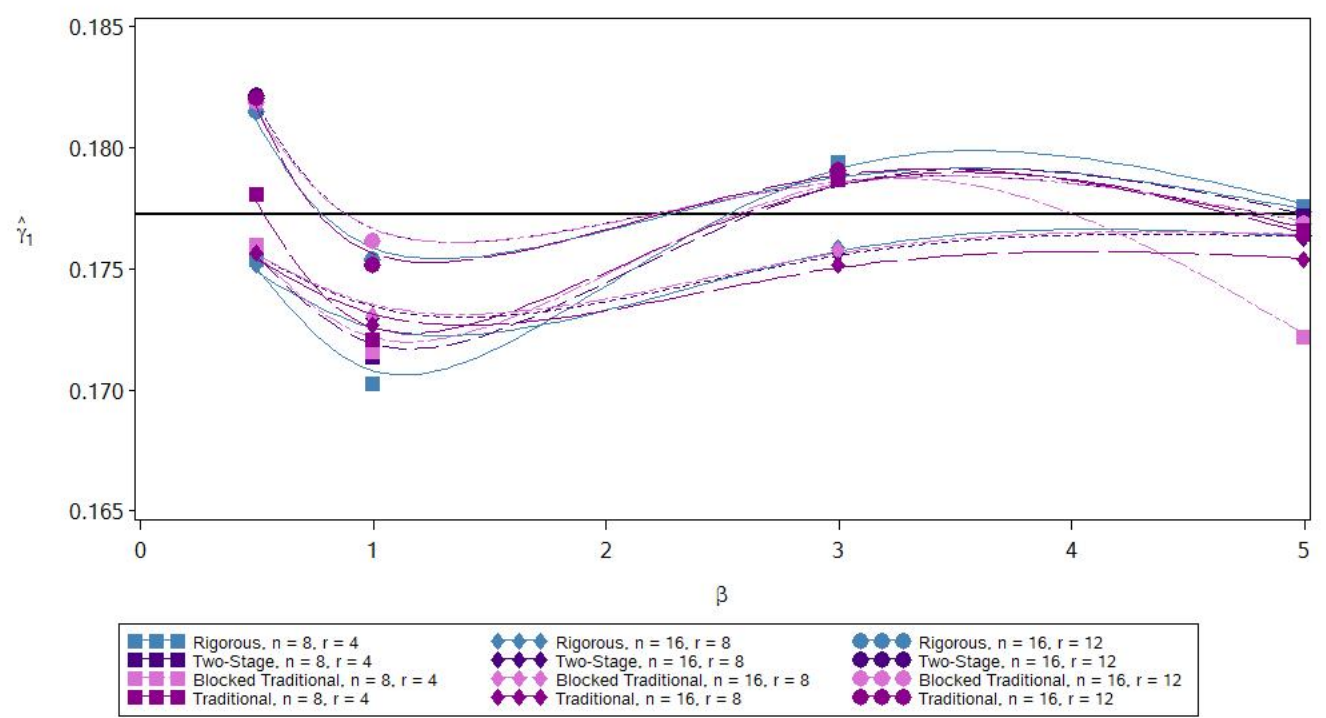

(b) $\gamma_{1}=0.35453, \sigma_{\rho}=0.2449$ and $\sigma_{\omega}=0.2$

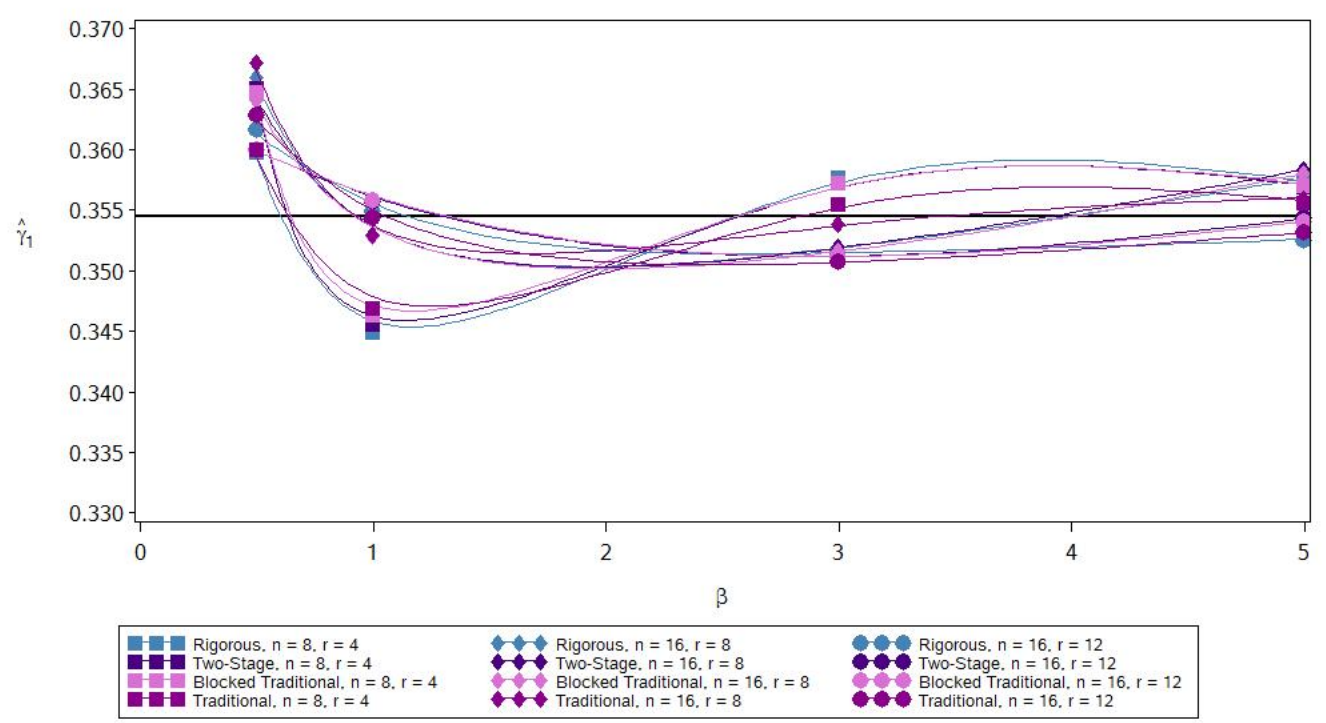


Figures 6.3 through 6.6 show that the NLMM, two-stage and traditional analyses produce similar estimates for $\gamma_{1}$. The bias of the estimates depends on the number of failures per test stand, $\beta$ and $\sigma_{\omega}$. Figure 6.7 contrasts the performance of the NLMM and two-stage methods in the estimation of $\sigma_{\rho}$. Note that the traditional analysis and the traditional analysis with fixed blocks do not estimate either $\sigma_{\rho}$ or $\sigma_{\omega}$. 
Figure 6.7: $\hat{\sigma}_{\rho}$

(a) $\sigma_{\rho}=0.1732$ for $\sigma_{\omega}=0.1$

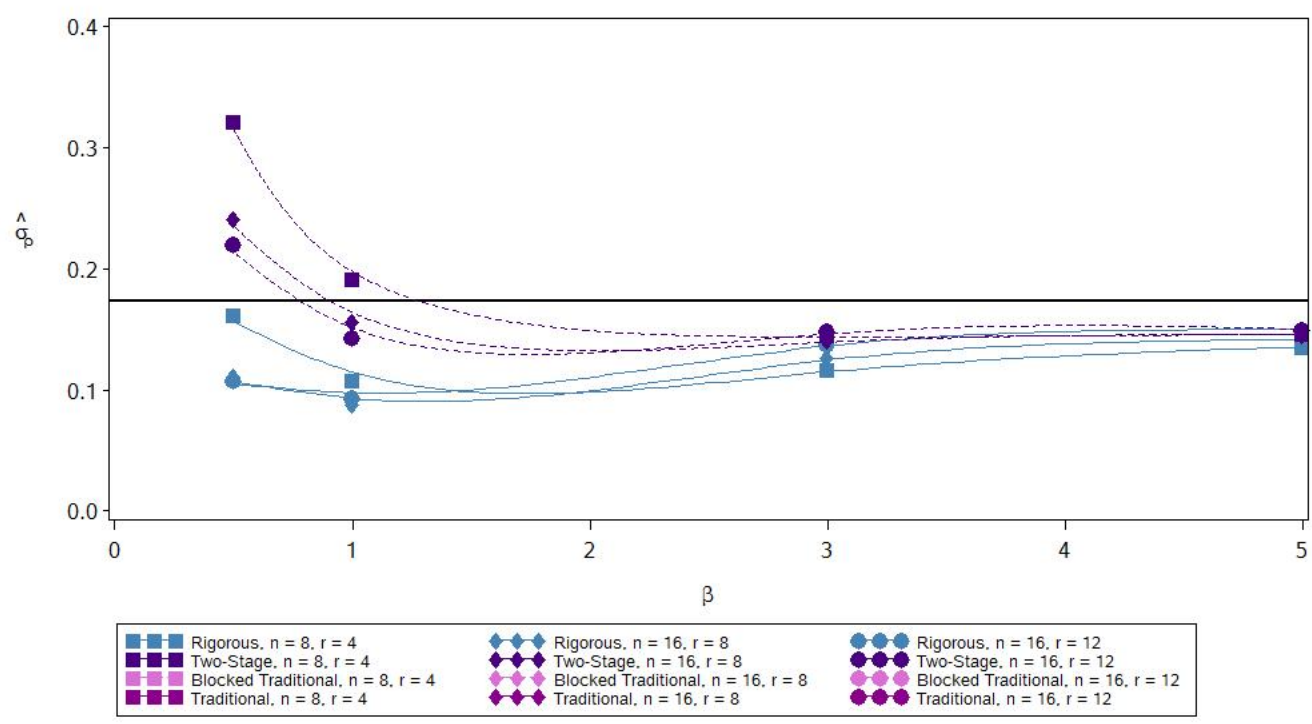

(b) $\sigma_{\rho}=0.2449$ for $\sigma_{\omega}=0.2$

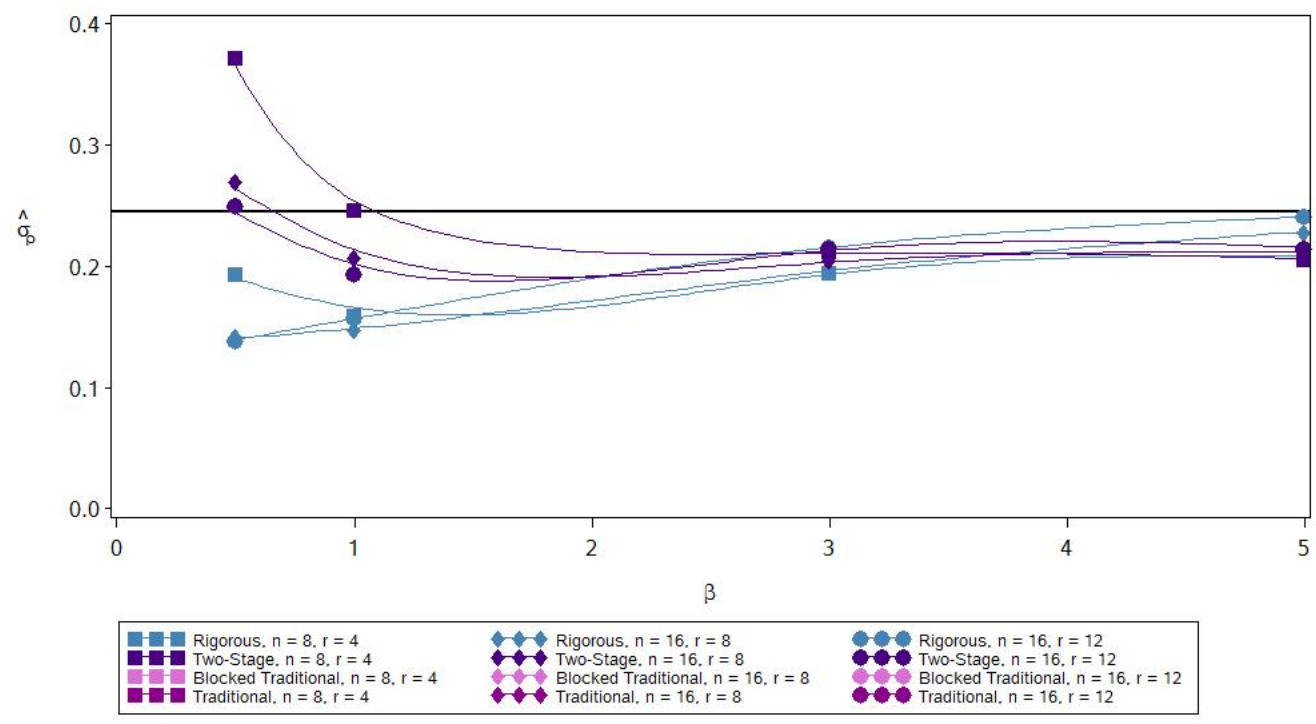


Figure 6.8 juxtaposes the performance of the estimation of $\sigma_{\omega}$ for the two-stage and NLMM analyses.

Figure 6.8: $\hat{\sigma}_{\omega}$

(a) $\sigma_{\omega}=0.1$ for $\sigma_{\rho}=0.1732$
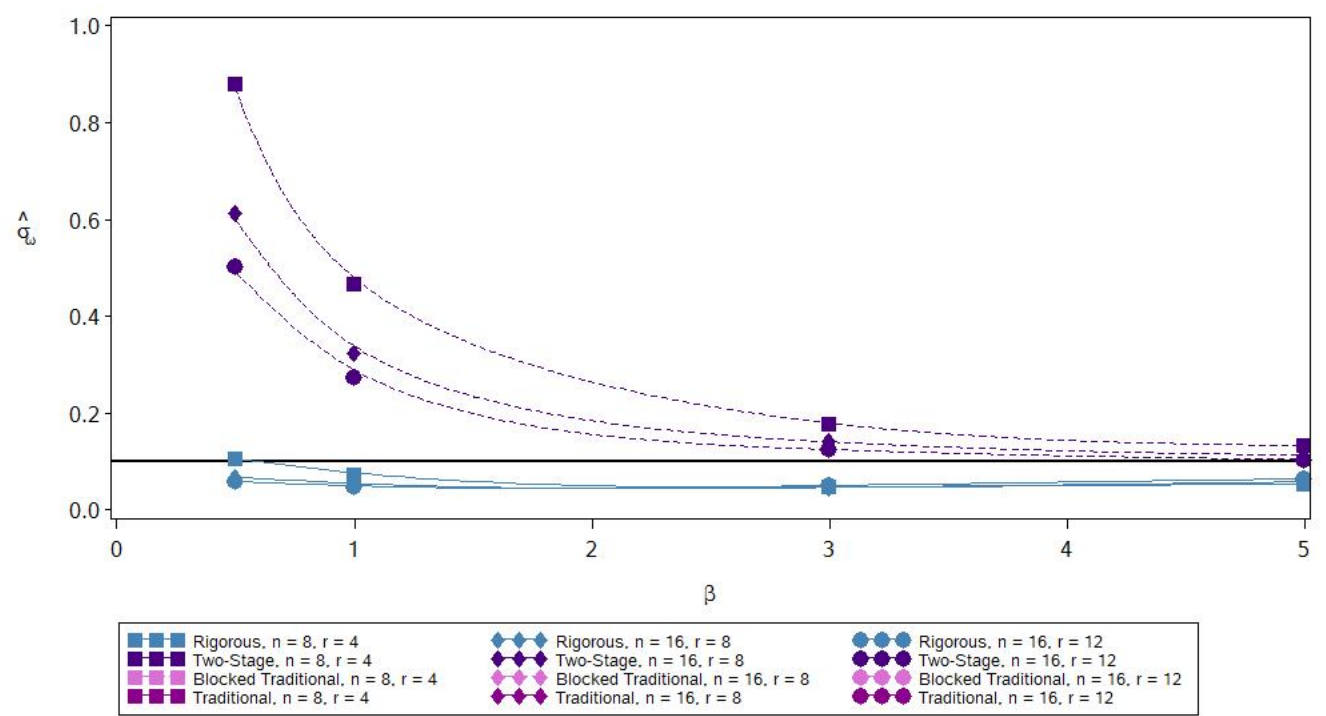

(b) $\sigma_{\omega}=0.2$ for $\sigma_{\rho}=0.2449$

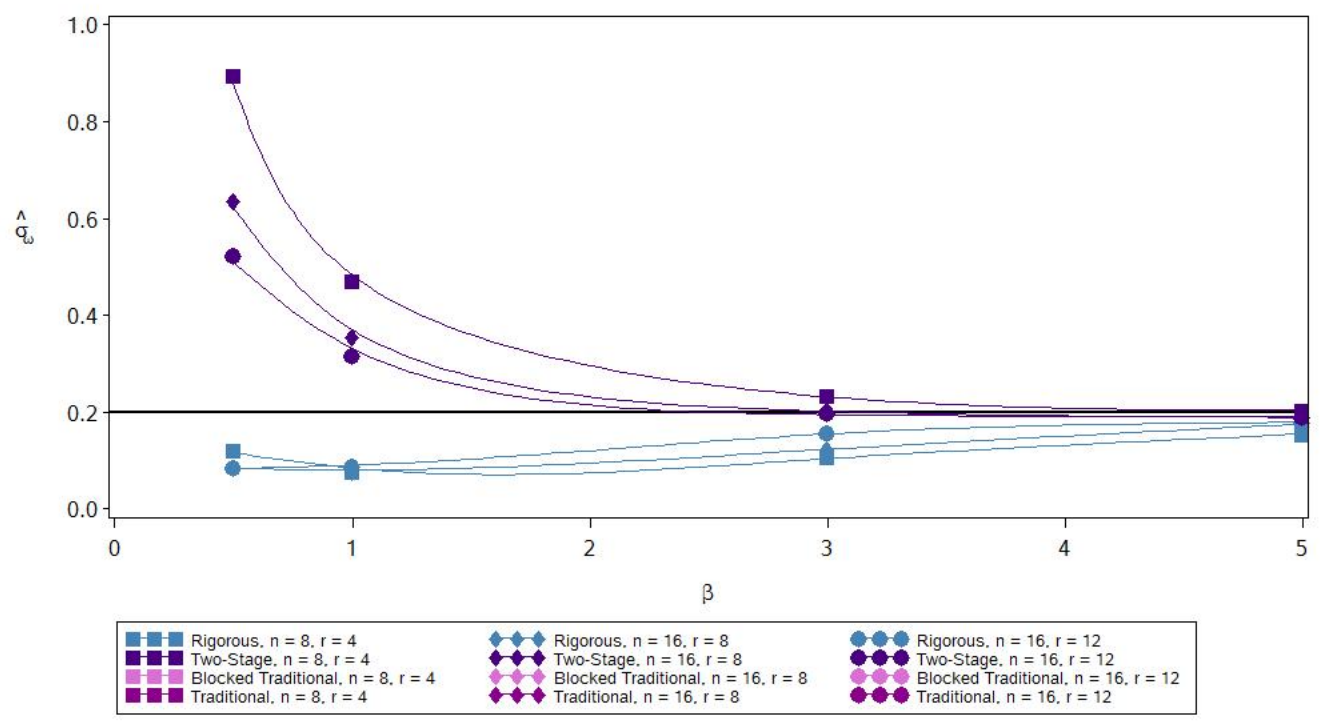


Figure 6.8 shows that the NLMM provides a much better estimate of $\sigma_{\omega}$ than the two-stage method. Figure 6.9 depicts the empirical error for testing $H_{0}: \gamma_{1}=0$ under a nominal type I error rate of 0.05 . For the NLMM method the simulations examine the power of two different testing methods. The first method is the likelihood ratio test and the second is the Wald test. 
Figure 6.9: Empirical Error Rate

(a) $\sigma_{\rho}=0.1732$ and $\sigma_{\omega}=0.1$

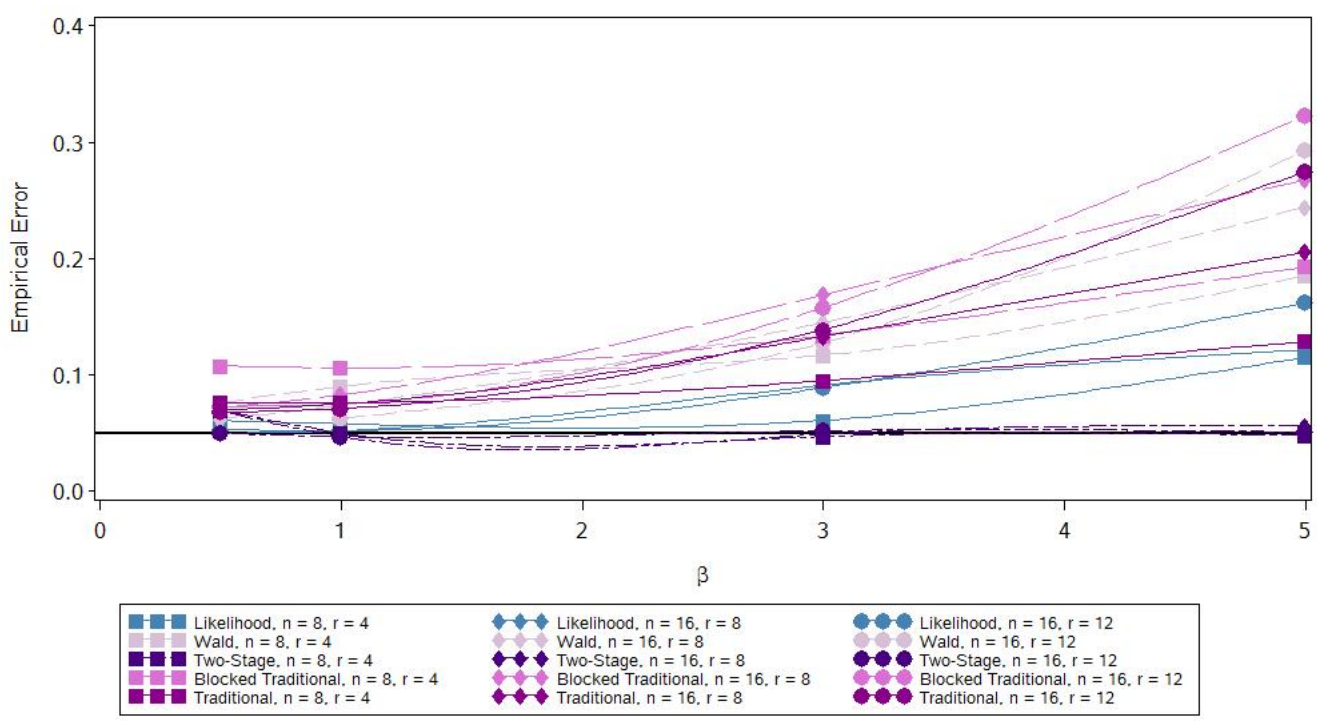

(b) $\sigma_{\rho}=0.2449$ and $\sigma_{\omega}=0.2$

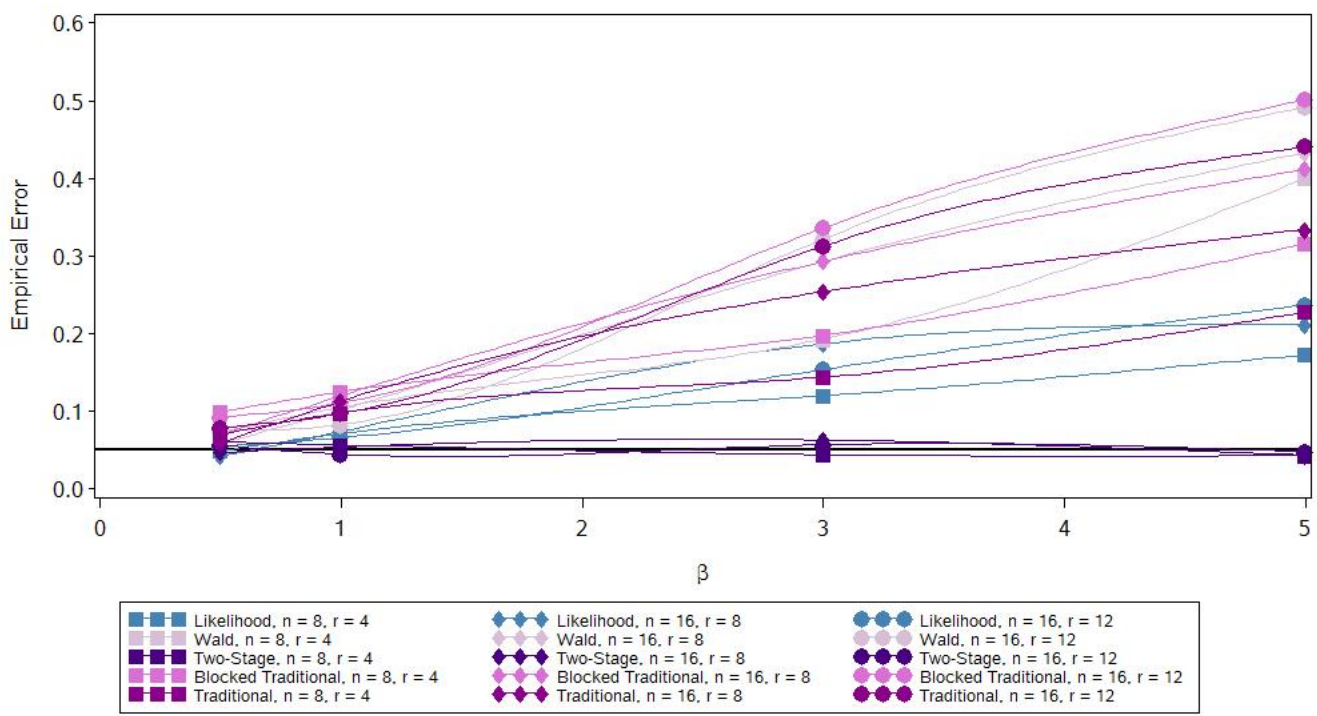


Figure 6.9 shows that the two-stage method preserves the nominal type I error rate. The likelihood ratio test for the NLMM has an empirical error rate that increases to 0.20 depending on the test stand error for large values of $\beta$. The Wald, traditional, and blocked traditional methods have very high empirical errors, particularly for $\beta=3$ and $\beta=5$. Figure 6.10 illustrates the empirical power of a test on $\gamma_{1}$ under a nominal power of 0.50 and a type I error rate of 0.05 . 
Figure 6.10: Nominal Power of 0.50

(a) $\sigma_{\rho}=0.1732$ and $\sigma_{\omega}=0.1$

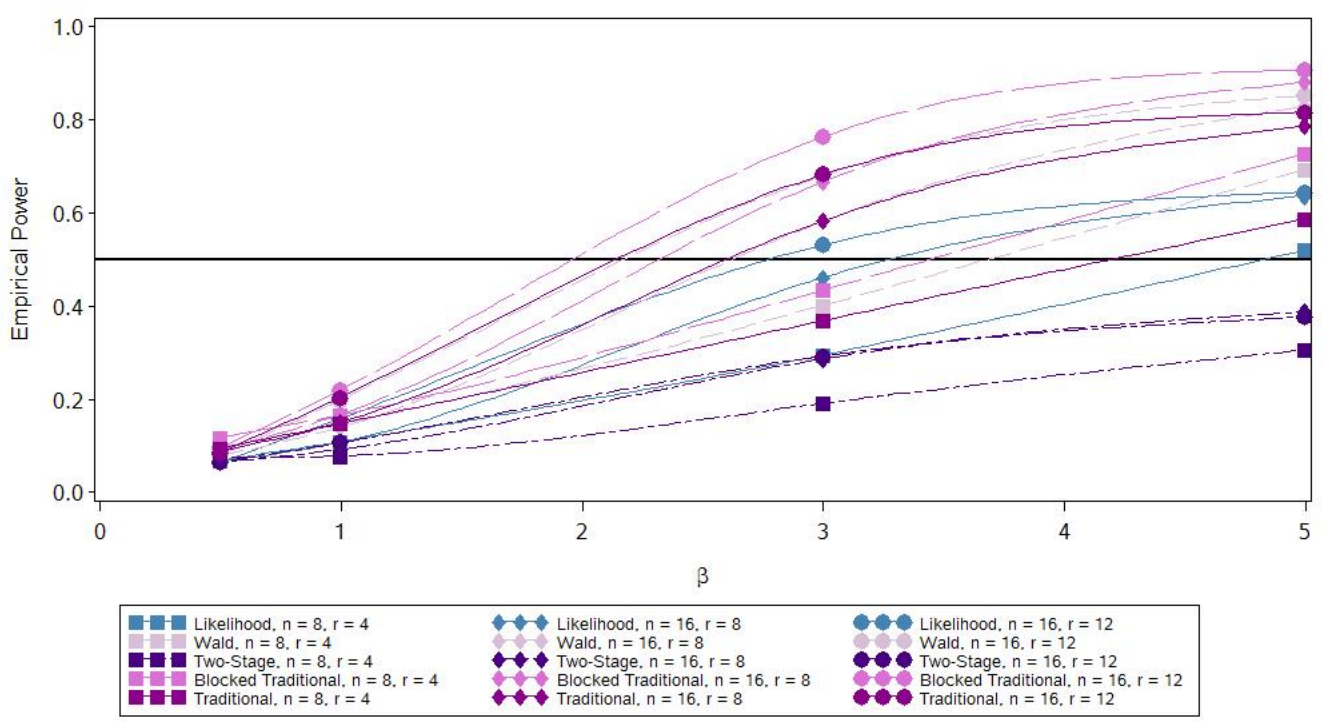

(b) $\sigma_{\rho}=0.2449$ and $\sigma_{\omega}=0.2$

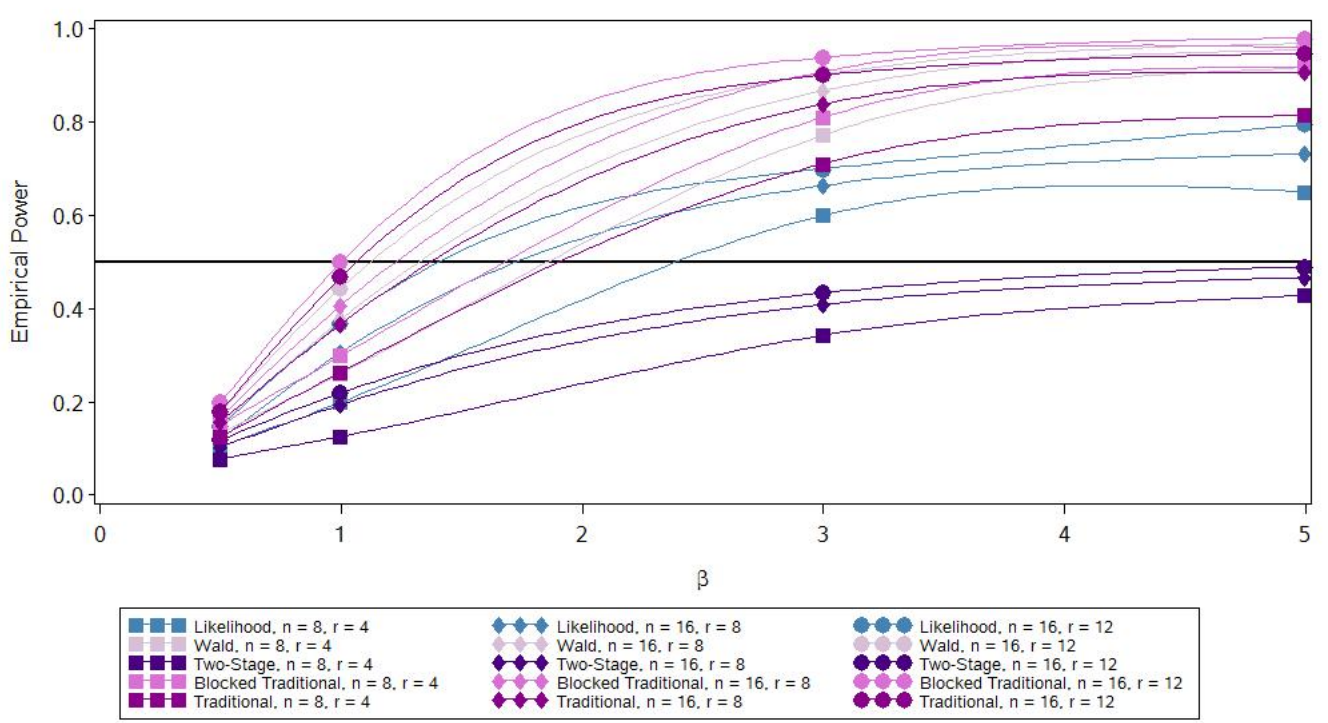


One should be cautious in comparing the empirical powers in Figure 6.10 with the nominal power of 0.50 . The nominal power is based on stage 2 model of the two-stage method. The shape parameter $\beta$ is estimated in stage 1 and does not appear in the stage 2 model. However, $\beta$ affects the variance of the Weibull distribution, as does $\sigma_{\rho}$. Since these parameters affect the variability of failure times, they also affect the power of a test. In order to understand the variability of failure times, simulations involving the random block with subsampling design were conducted for both censored and uncensored data. Appendix D contains these simulations, which provide insight into the variance of the Weibull distribution. Figure 6.11 shows the empirical power of the five methods for a nominal power of 0.80 and a nominal type I error rate of 0.05 . 
Figure 6.11: Nominal Power of 0.80

(a) $\sigma_{\rho}=0.1732$ and $\sigma_{\omega}=0.1$

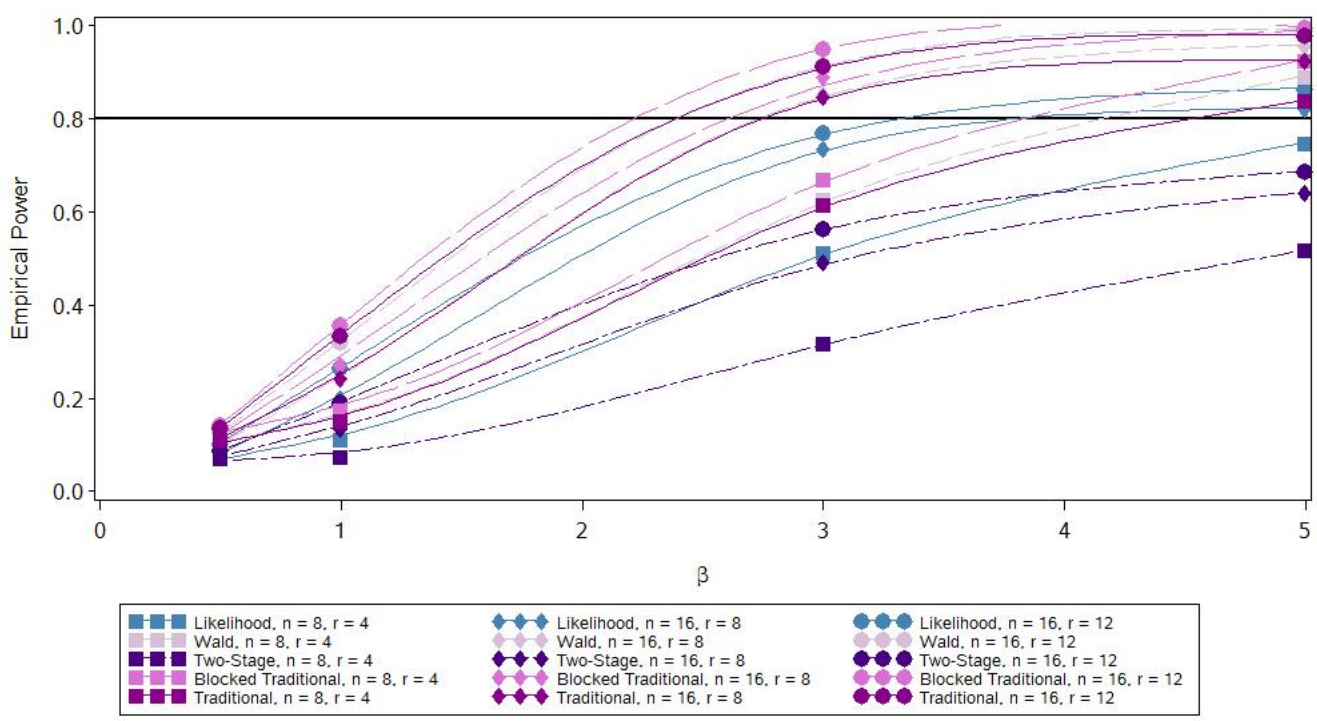

(b) $\sigma_{\rho}=0.2449$ and $\sigma_{\omega}=0.2$

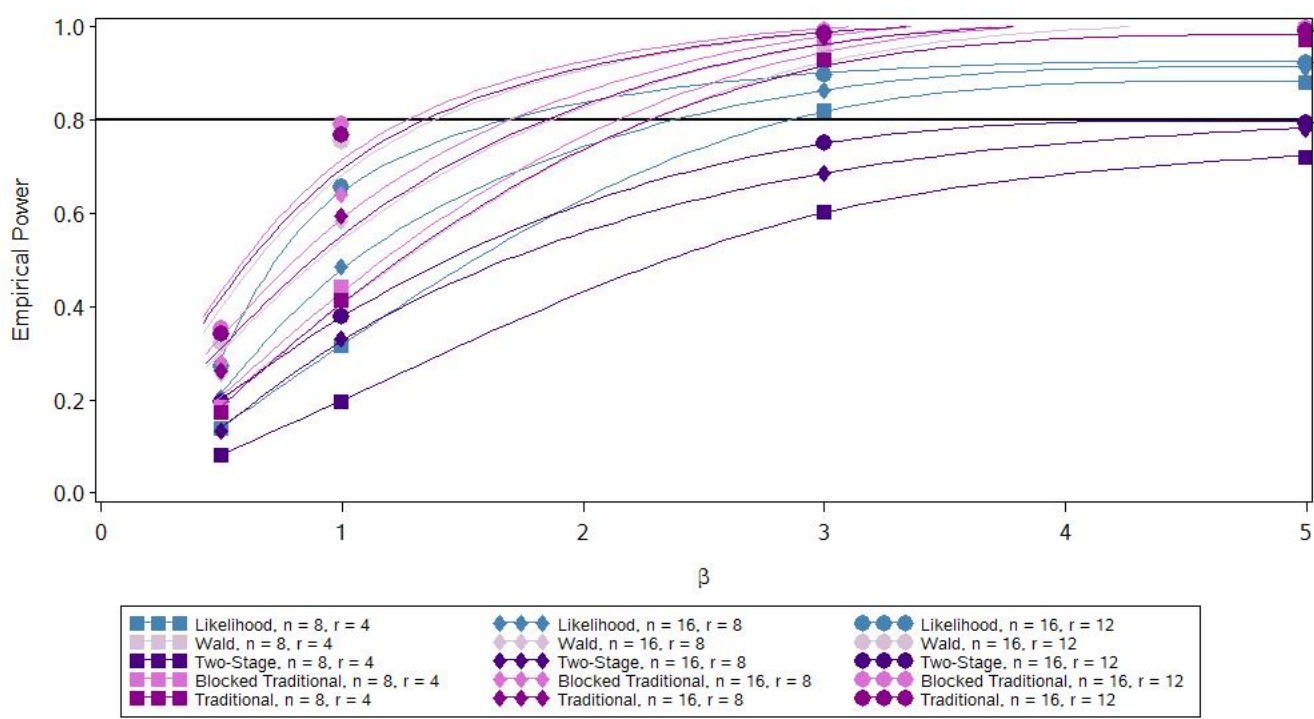


The results of Figure 6.11 are similar to those of Figure 6.10. Figure 6.12 shows the empirical power for the five methods under a nominal power of 0.90 and a nominal type I error rate of 0.05 . 
Figure 6.12: Nominal Power of 0.90

(a) $\sigma_{\rho}=0.1732$ and $\sigma_{\omega}=0.1$

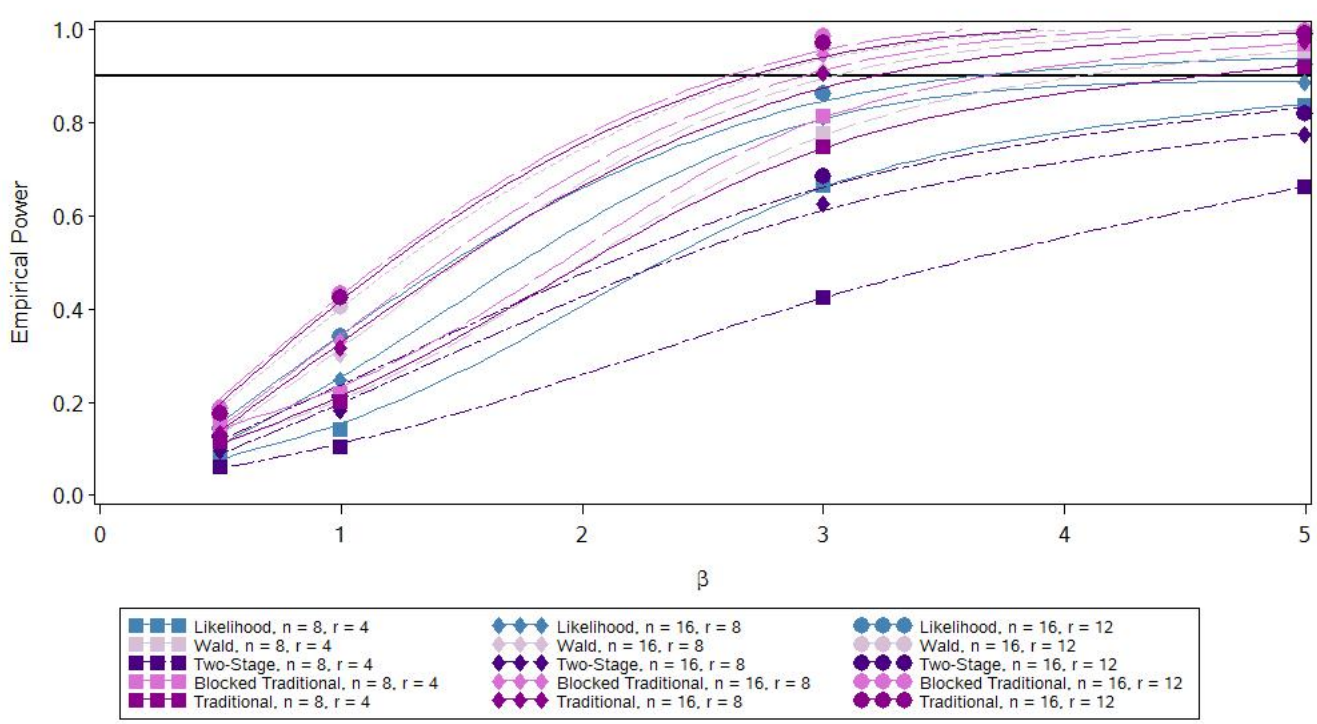

(b) $\sigma_{\rho}=0.2449$ and $\sigma_{\omega}=0.2$

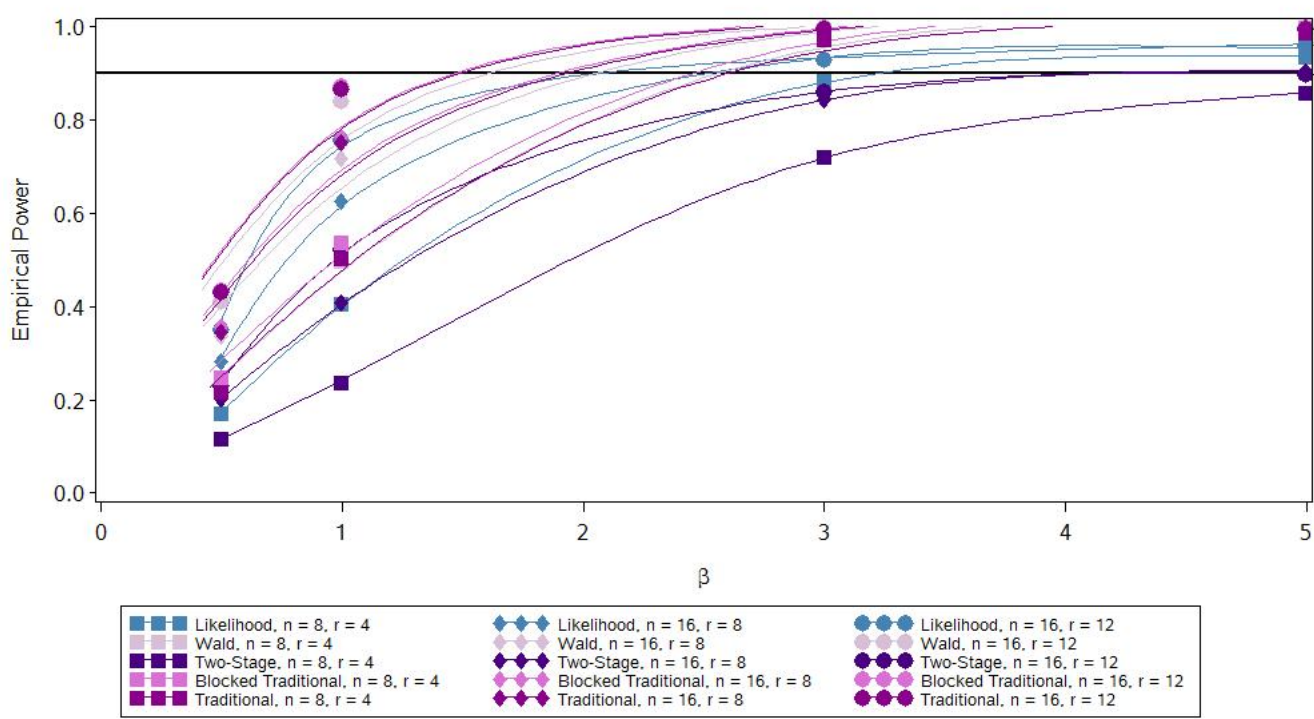




\subsection{Simulation Study for Percentiles}

This simulation study compares the performance of the NLMM and traditional analyses for estimating and performing inferences on $t_{0.1}$. Since the traditional analysis with blocks treats the blocks as fixed, it is not included in this study. Furthermore, the two-stage method is excluded as it does not allow for inferences on $t_{p}$. The battery example from Chapter 4 provides the background for this simulation study. In this scenario, batteries come from three batches (random blocks) and each batch feeds three chambers with temperatures of 15 ${ }^{\circ} \mathrm{F}, 70^{\circ} \mathrm{F}$, and $125^{\circ} \mathrm{F}$ respectively. Thus, the experimental scenario is a randomized complete block design. Each chamber contains $n$ batteries and type II censoring is used after the first $r$ failures.

The failure times follow a Weibull distribution with shape parameter $\beta=5,3,1$, and 0.5 , and $\log$-scale parameter $\log \left(\eta_{i j}\right)=\mu_{i j}=\gamma_{0}+\gamma_{1} x_{i j}+\rho_{i}+\omega_{i j}$ where $\rho_{i} \sim N\left(0, \sigma_{\rho}^{2}\right)$ and $\omega_{i j} \sim N\left(0, \sigma_{\omega}^{2}\right)$ are independent. This simulation study explores three combinations of $n$ and $r: n=8$ and $r=4, n=16$ and $r=8$, and $n=16$ and $r=12$. Simulations are completed for $\sigma_{\rho}=0.08$ with $\sigma_{\omega}=0.1$, and $\sigma_{\rho}=0.2828$ with $\sigma_{\omega}=0.2$. Under the null model $\gamma_{0}=5.5$ and $\gamma_{1}=-0.007$, which gives $t_{p 0}(x)=\exp \left[5.5-0.007 x+\frac{\Phi_{S E V}^{-1}(p)}{\beta}\right]$. This simulation study investigates $p=0.1$ for $x=70$; thus, under the null model $t_{0.1,0}(70)=$

$\exp \left[5.5-0.007(70)+\frac{\Phi_{S E V}^{-1}(0.1)}{\beta}\right]$. Under the alternative model, $15 \%$ of batteries fail by $t_{0.1,0}(70)$. Moreover, there exists a one-to-one correspondence between $t_{p}$ and $\gamma_{1}$ : 


$$
\gamma_{1}=\frac{1}{x}\left[\log \left(t_{p}(x)\right)-5.5-\frac{\Phi_{S E V}^{-1}(p)}{\beta}\right] .
$$

As discussed in the preceding paragraph, $t_{0.1,0}(70)$ corresponds to $\gamma_{1}=-0.007$ under the null model. Under of alternative model, $15 \%$ of failures by $t_{0.1,0}(70)$ corresponds to

$$
\gamma_{1}=-0.007-\frac{\Phi_{S E V}^{-1}(0.1)-\Phi_{S E V}^{-1}(0.15)}{70 \beta} .
$$

The simulation study contains 1000 replicates and the NLMM analysis uses 5 quadrature points. 
Figure 6.13 shows the ratio of $\hat{t}_{0.1}(70)$ to $t_{0.1}(70)$ under the null model.

Figure 6.13: $\frac{\hat{t}_{0.1}(70)}{t_{0.1}(70)}$ Under the Null Model

(a) $\sigma_{\rho}=0.08, \sigma_{\omega}=0.1$

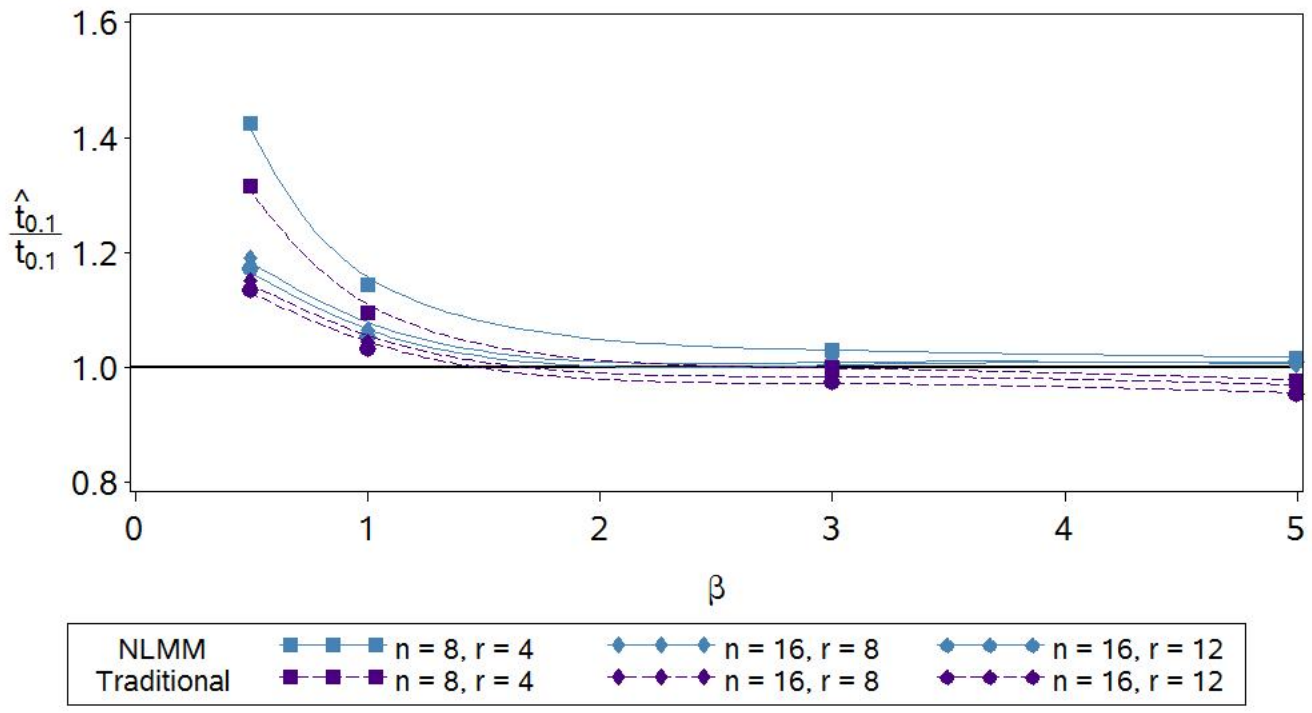

(b) $\sigma_{\rho}=0.2828, \sigma_{\omega}=0.2$

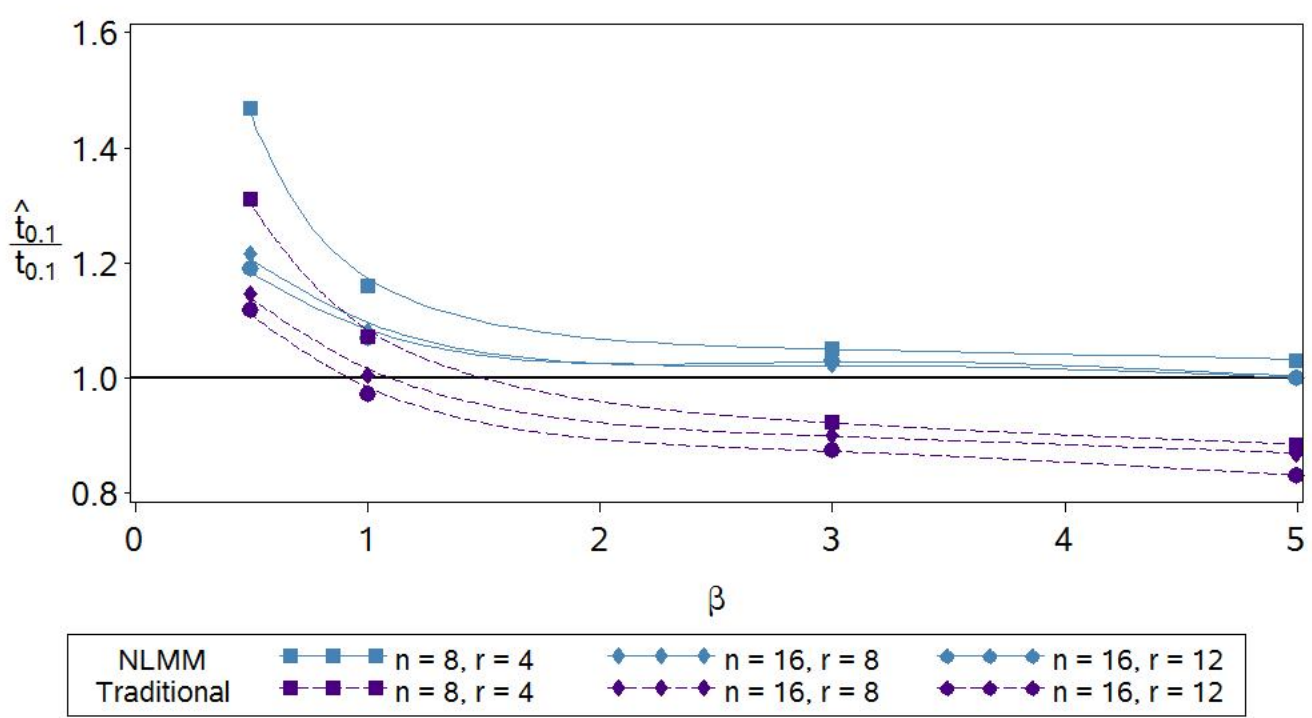


Figure $6.13 \mathrm{a}$ shows that both methods overestimate $t_{0.1}$ for small values of $\beta$. The performance of the traditional method depends on the test stand and random block errors; whereas, the performance of the NLMM method does not. Figure 6.14 shows the ratio of $\hat{t}_{0.1}(70)$ to $t_{0.1}(70)$ under the alternative model. 
Figure 6.14: $\frac{\hat{t}_{0.1}(70)}{t_{0.1}(70)}$ Under the Alternative Model

(a) $\sigma_{\rho}=0.08, \sigma_{\omega}=0.1$

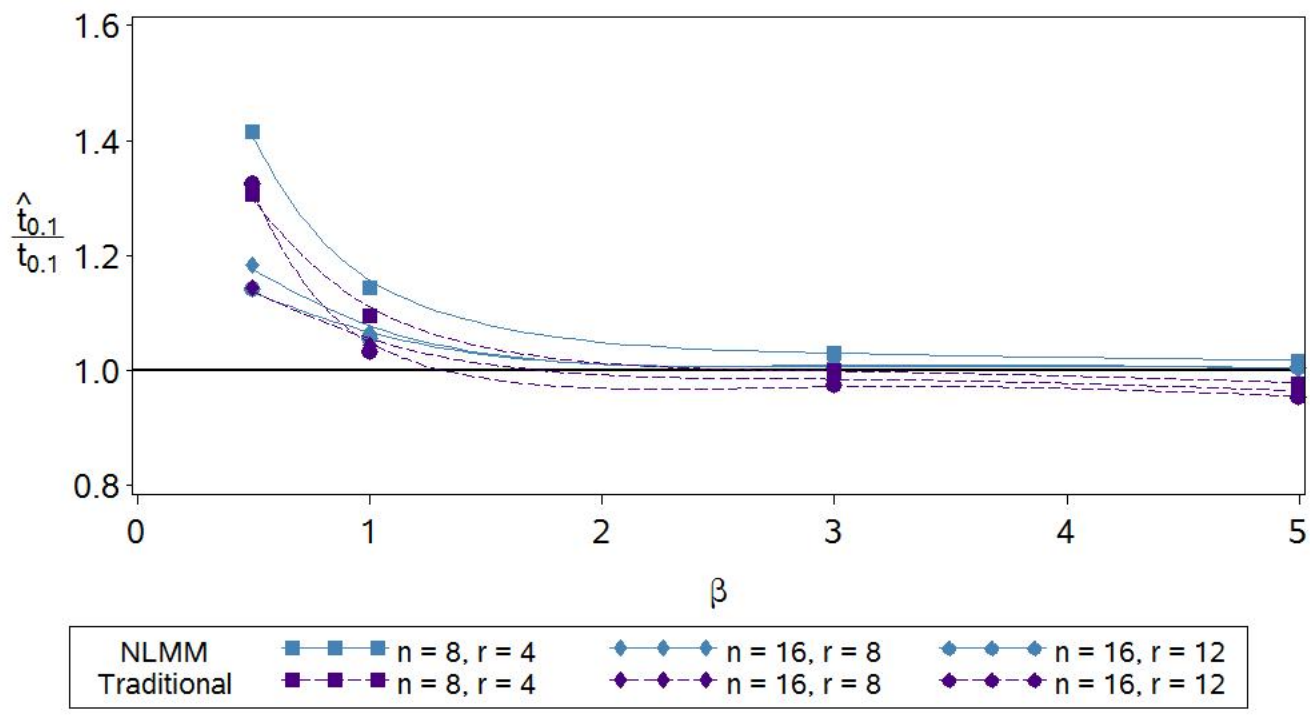

(b) $\sigma_{\rho}=0.2828, \sigma_{\omega}=0.2$

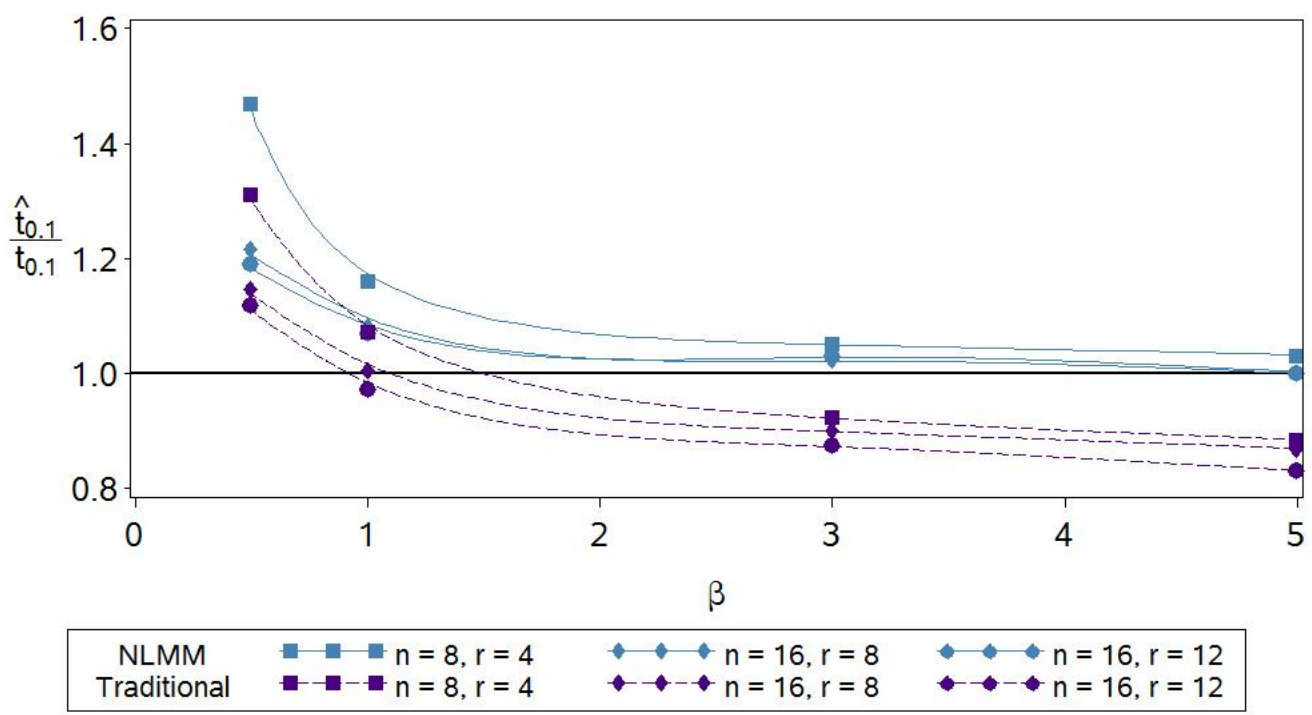


The results under the alternative model depicted in Figure 6.14 are very similar to the results in Figure 6.13 under the null model. Figure 6.15 displays the empirical error for the test $H_{0}: t_{0.1}(70)=t_{0.1,0}(70)$ vs. $H_{0}: t_{0.1}(70) \neq t_{0.1,0}(70)$. In the NLMM analysis the Wald method is used to test the hypotheses. Although a test based on the likelihood is also desired, more research is needed to implement this test. Hong et al. (2008b) provides insight into inference on percentiles; however, the current situation involves the added complexity of random effects. 
Figure 6.15: Empirical Error Rate for Testing $t_{0.1}$

(a) $\sigma_{\rho}=0.08, \sigma_{\omega}=0.1$

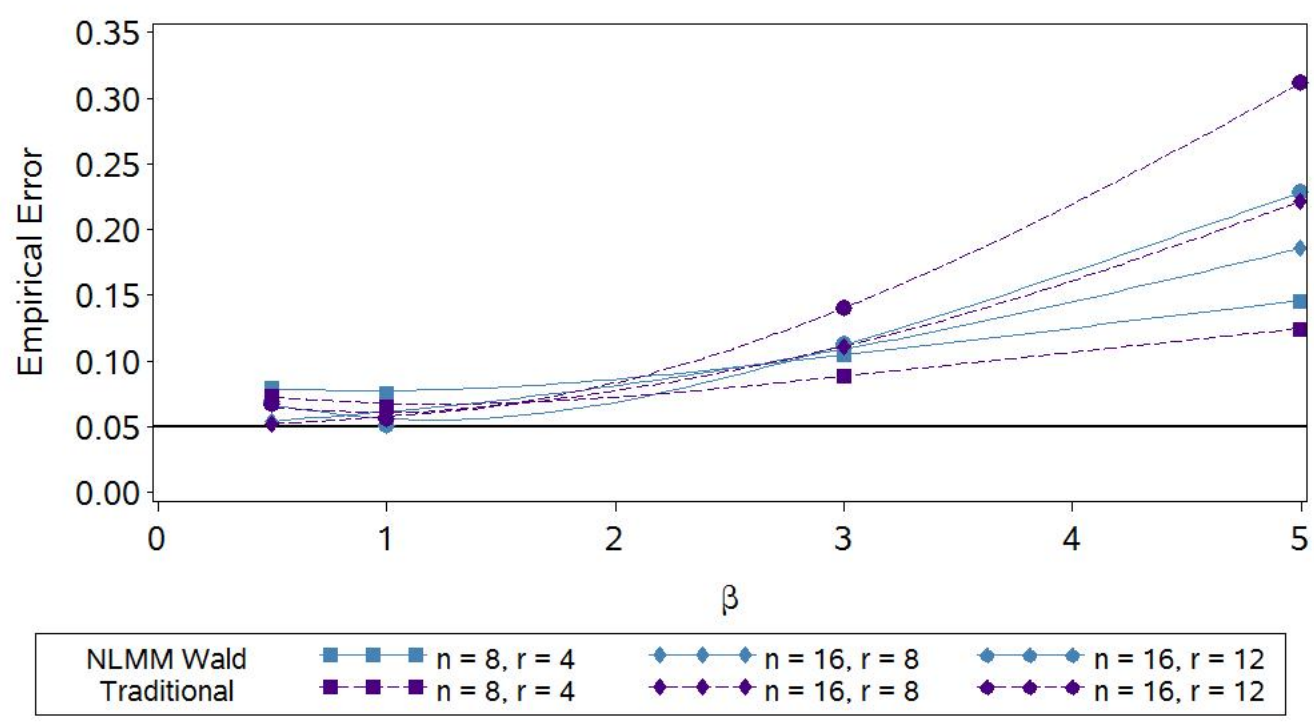

(b) $\sigma_{\rho}=0.2828, \sigma_{\omega}=0.2$

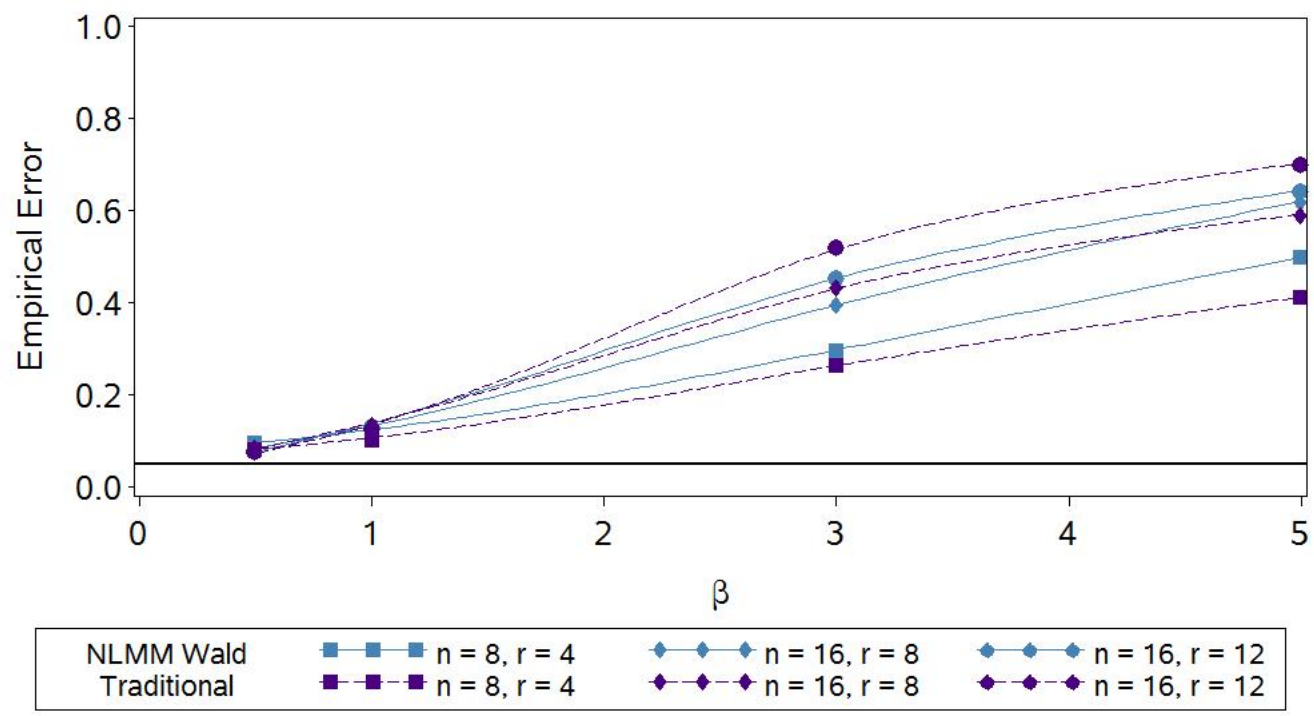


Figure 6.15 shows that the Wald approach and the traditional analysis perform similarly in terms of the empirical error. Figure 6.16 illustrates the empirical power of the hypothesis test when data is generated using the alternative model. 
Figure 6.16: Empirical Power for Testing $t_{0.1}$

(a) $\sigma_{\rho}=0.08, \sigma_{\omega}=0.1$

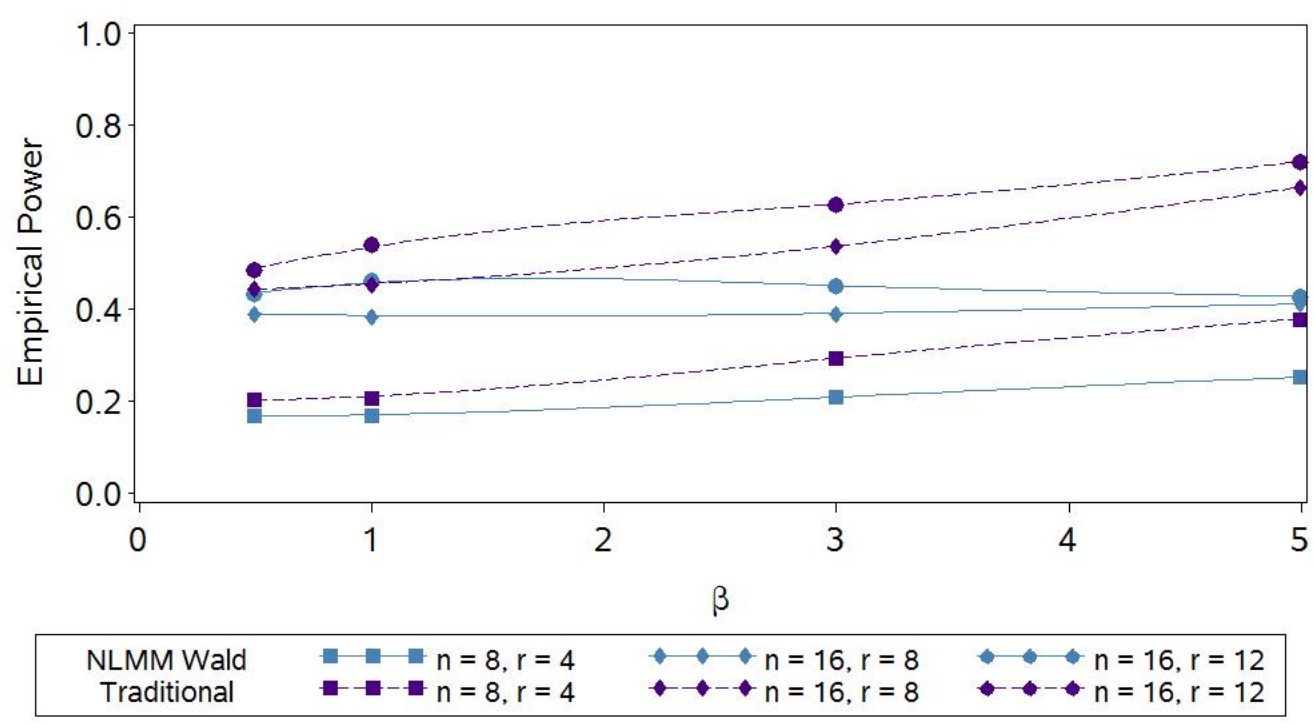

(b) $\sigma_{\rho}=0.2828, \sigma_{\omega}=0.2$

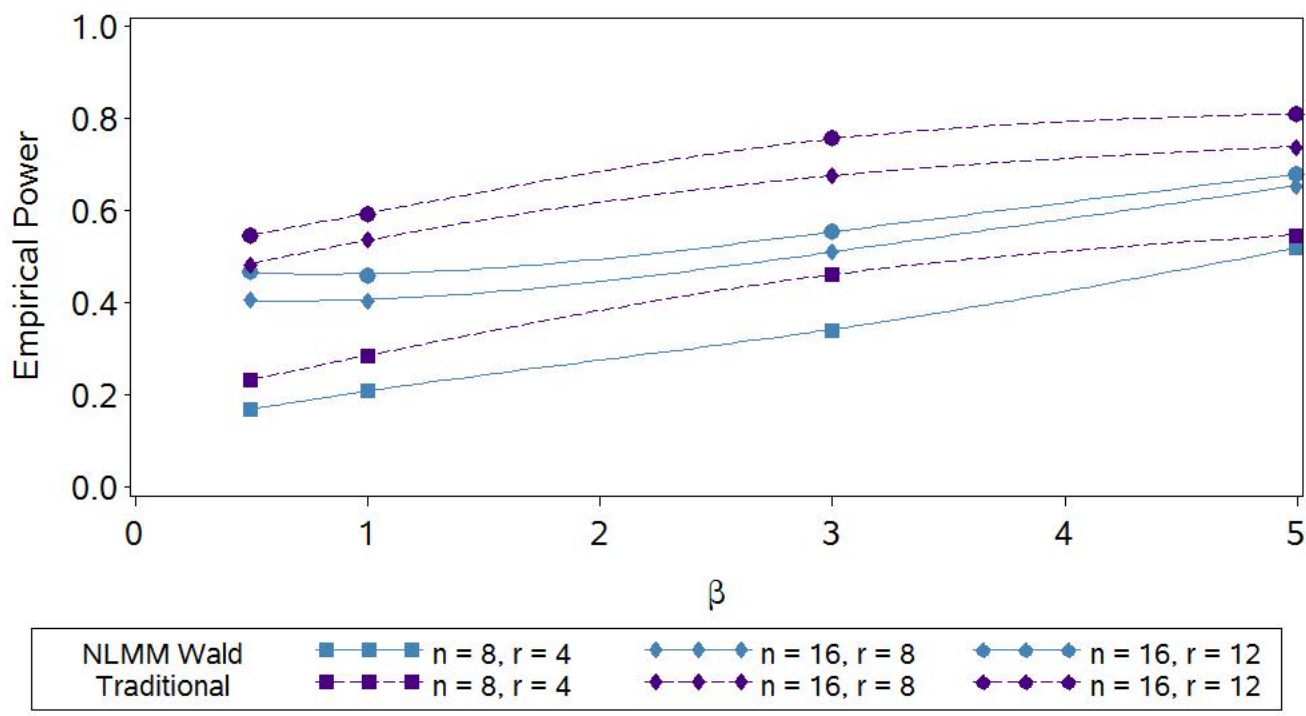

The above figure shows that the two methods perform similarly in terms of empirical power. 


\subsection{Conclusions from Simulation Studies}

Results from the simulation study in Section 6.1 suggest using the NLMM for parameter estimation and the two-stage method for testing factor effects. Of the four methods the NLMM analysis tends to have the smallest bias. Furthermore, the bias of estimates from the NLMM method are mostly invariant to changes in $\beta$. The two-stage method preserves the nominal type I error rate of 0.05 in all cases studied, which is an important consideration in testing. Moreover, the empirical power of the two-stage method approaches the nominal power as the sample size increases. Nevertheless, since the nominal power was based on the two-stage method this is not unexpected.

The simulation study in Section 6.2 suggests that for percentiles the Wald approach and the traditional approach produce similar results. The NLMM is recommended for percentile estimation since its behavior is robust to the test stand and random block errors. Both methods have inflated empirical error rates, particularly for large values of $\beta$. Section 6.1 demonstrates that while the likelihood approach to testing may not preserve the nominal type I error rate, it does a better job than the Wald or the traditional approaches. Perhaps a likelihood ratio test for percentiles would perform better. 


\section{Chapter 7}

\section{Conclusions and Future Work}

\subsection{Conclusions}

The research in this dissertation emphasizes the importance of incorporating the experimental protocol into the analysis of reliability data. While incorporating restrictions on randomization into the analysis may seem obvious from the design of experiments standpoint, current analyses of reliability experiments typically do not account for these restrictions. This dissertation helps to bridge the gap between design of experiments and reliability analysis.

The two-stage method proposed in Chapter 4 can be easily implemented by practitioners with their current software and is recommended for testing factor effects. The two-stage method estimates the Weibull shape parameter, $\beta$, in stage 1 , while factor effects are estimated in 
stage 2. This staged estimation allows tests for factor effects that preserve the nominal type I error rate for all values of the Weibull shape parameter $\beta$. Limitations of the two-stage method include overestimation of $\beta$ and the inability to perform closed form inferences on functions of parameters that contain both $\beta$ and a parameter estimated in stage 2 .

The NLMM analysis proposed in Chapter 5 can be implemented using SAS or R. Nevertheless, some practitioners may not be able to implement this method. Not all practitioners have access to SAS, and implementing the analysis in $\mathrm{R}$ requires extensive programing. The NLMM method is recommended for parameter estimation. Likelihood based inferences are preferred over the Wald inferences, as the likelihood based inferences do a better job of controlling the type I error rate and are known to have better properties for small sample sizes.

\subsection{Future Work}

There are many possibilities for extending the work of this dissertation. Possibilities include extending the work on the random blocks with subsampling design or extending the work to other designs. Ideas for research for the random block with subsampling design include implementing likelihood based confidence intervals for parameters in the NLMM approach, as well as implementing likelihood based confidence intervals and tests for percentiles. Although the theory for likelihood based confidence intervals is straightforward, the implementation is not. Current software does not appear to have built in tools for computing likelihood based 
confidence intervals for nonlinear models with random effects.

Another area that needs additional work is the power of detecting a factor effect. The nominal powers based on stage 2 of the two-stage method do not depend on $\beta$ or $\sigma_{\rho}$. However, these parameters affect the variance of the Weibull distribution and hence the power of tests. Further research could be performed to quantify how the various parameters impact the power.

Investigating the sample sizes needed to obtain reasonable estimates of the test stand and random block errors is also necessary. This investigation involves examining the effect of increasing the number of blocks and the number of test stands per block, as well as the number of items and failures per test stand.

Although the two-stage and NLMM analyses proposed apply to designs with random blocks and subsamples in general, the simulations performed all examined randomized complete block designs where each test stand had the same number of items and failures. Simulation studies examining the properties of the proposed methods for other block designs is a natural extension to the simulations in this dissertation.

Industrial experiments often involve split-plots, so a highly relevant extension would be to extend the two-stage and NLMM methods to the split-plot design. Additional possibilities include exploring the extension of these methods to other members of the log-location-scale family of distributions or generalizing these methods to apply to the entire log-location-scale family. 


\section{Bibliography}

[1]

[2] Abramowitz, M., And Stegun, I. A. Handbook of Mathematical Functions with Formulas, Graphs, and Mathematical Tables. Dover, New York, 1964.

[3] Billmann, B. R., Antle, C. E., And Bain, L. J. Statistical inference from censored weibull samples. Technometrics 14, 4 (November 1972), 831-840.

[4] Cacciari, M., Mazzanti, G., and Montanari, G. C. Comparison of maximum likelihood unbiasing methods for the estimation of the weibull parameters. IEEE Transactions on Dielectrics and Electrical Insulation 3, 1 (February 1996), 18-27.

[5] Casella, G., And Berger, R. L. Statistical Inference, 2 ed. Duxbury, Pacific Grove, CA, 2002.

[6] Feiveson, A. H., And Kulkarni, P. M. Reliability of space-shuttle pressure vessels with random batch effects. Technometrics 42, 4 (November 2000), 332-344. 
[7] Freeman, L. J. Statistical Methods for Reliability Data from Designed Experiments. Unpublished PhD dissertation, Virginia Tech, 2010.

[8] Freeman, L. J. A cautionary tale: Small sample size concerns for grouped lifetime data. Quality Engineering 23, 2 (June 2011), 134-141.

[9] Freeman, L. J., And Vining, G. G. Reliability data analysis for life test experiments with subsampling. Journal of Quality Technology 42, 3 (July 2010), 233-241.

[10] Freeman, L. J., And Vining, G. G. Reliability data analysis for life test designed experiments with sub-sampling. Quality and Reliability Engineering International (2012), To Appear.

[11] Genschel, U., And Meeker, W. Q. A comparison of maximum likelihood and median-rank regression for weibull estimation. Quality Engineering 22, 4 (December 2010), 236-255.

[12] Gerstle, Jr., F. P., And Kunz, S. C. Prediction of long-term failure in kevlar 49 composites. In Long-Term Behavior of Composites, ASTM STP 813, T. K. O'Brien, Ed. American Society for Testing and Materials, Philadelphia, PA, 1983, pp. 263-292.

[13] Graybill, F. A. Theory and Application of the Linear Model. Duxbury, Pacific Grove, CA, 1976.

[14] Hamada, M. Using statistically designed experiments to improve reliability and to achieve robust reliability. IEEE Transactions on Reliability 44, 2 (June 1995), 206-215. 
[15] Hong, Y., Meeker, W. Q., And Escobar, L. A. Avoiding problems with normal approximation confidence intervals for probabilities. Technometrics 50, 1 (February 2008), 64-68.

[16] Hong, Y., Meeker, W. Q., And Escobar, L. A. Relationship between confidence intervals for failure probabilities and life time quantiles. IEEE Transactions on Reliability 57, 2 (June 2008), 260-266.

[17] Klein, J. P., And Moeschberger, M. L. Survival Analysis: Techniques for Censored and Truncated Data, 2 ed. Springer, New York, 2003.

[18] Kutner, M. H., Nachtsheim, C. J., Neter, J., And Li, W. Applied Linear Statistical Models, 5 ed. McGraw-Hill/Irwin, New York, 2005.

[19] Lawless, J. F. Statistical methods in reliability. Technometrics 25, 4 (November 1983), 305-316.

[20] Lawless, J. F. Statistical Models and Methods for Lifetime Data, 2 ed. John Wiley and Sons, Hoboken, NJ, 2003.

[21] León, R. V., Li, Y., Guess, F. M., and Sawhney, R. S. Effect of not having homogeneous test units in accelerated life tests. Journal of Quality Technology 41, 3 (July 2009), 241-246.

[22] Littell, R. C., Milliken, G. A., Stroup, W. W., Wolfinger, R. D., And Schabenberger, O. SAS(R) for Mixed Models. SAS Institute Inc., Cary, NC, 2006. 
[23] McCulloch, C. E., And Searle, S. R. Generalized, Linear, and Mixed Models. John Wiley and Sons, Hoboken, NJ, 2001.

[24] Meeker, W. Q., And Escobar, L. A. Statistical Methods for Reliability Data. John Wiley and Sons, Hoboken, NJ, 1998.

[25] Montanari, G. C., Mazzanti, G., Cacciari, M., and Fothergill, J. C. Optimum estimators for the weibull distribution of censored data singly-censored tests. IEEE Transactions on Dielectrics and Electrical Insulation 4, 4 (August 1997), 462-469.

[26] Montgomery, D. C. Design and Analysis of Experiments, 6 ed. John Wiley and Sons, Hoboken, NJ, 2005.

[27] Nelson, W. Accelerated Testing: Statistical Models, Test Plans, and Data Analyses. John Wiley and Sons, Hoboken, NJ, 1990.

[28] Olteanu, D., and Freeman, L. The evaluation of median-rank regression and maximum likelihood estimation techniques for a two-parameter weibull distribution. Quality Engineering 22, 4 (December 2010), 256-272.

[29] Pinheiro, J. C., And Bates, D. M. Approximations to the log-likelihood function in the nonlinear mixed-effects model. Journal of Computational and Graphical Statistics 4, 1 (March 1995), 12-35. 
[30] Pinheiro, J. C., And ChaO, E. C. Efficient laplacian and adaptive gaussian quadrature algorithms for multilevel generalized linear mixed models. Journal of Computational and Graphical Statistics 15, 1 (March 2006), 58-81.

[31] Raudenbush, S. W., Yang, M.-L., and Yosef, M. Maximum likelihood for generalized linear models with nested random effect via high-order, multivariate laplace approximation. Journal of Computational and Graphical Statistics 9, 1 (March 2000), $141-157$.

[32] Ross, R. Formulas to describe the bias and standard deviation of the ml-estimated weibull shape parameter. IEEE Transactions on Dielectrics and Electrical Insulation 1, 2 (April 1994), 247-253.

[33] Ross, R. Bias and standard deviation due to weibull parameter estimation for small data sets. IEEE Transactions on Dielectrics and Electrical Insulation 3, 1 (February 1996), 28-42.

[34] SAS Institute Inc. SAS/STAT(R) 9.2 User's Guide. SAS Institute Inc., Cary, NC, 2008.

[35] Thoman, D. R., Bain, L. J., And Antle, C. E. Inferences on the parameters of the weibull distribution. Technometrics 11, 3 (August 1969), 445-460.

[36] Zelen, M. Experiments in life testing. Technometrics 1, 3 (August 1959), 269-288. 


\title{
Appendix A
}

\section{Two-Stage Simulation Study Tables}

\author{
and Figures
}

\section{A.1 Additional Tables for the Two-Stage Simulation Study}

Tables A.1 - A.3 contain additional results for the simulation study in Chapter 3. 
Jennifer L. K. Kensler Appendix A. Two-Stage Simulation Study Tables and Figures 135

Table A.1: Simulation Results Power 0.80: $n=8, r=4$

\begin{tabular}{|c|c|c|c|c|c|c|c|}
\hline & \multirow[b]{2}{*}{ Actual } & $\gamma_{0}$ & $\gamma_{1}$ & \multirow[t]{2}{*}{$\beta$} & \multirow{2}{*}{$\frac{\sigma_{\rho}}{2}$} & $\sigma_{\omega}$ & Power \\
\hline & & 15 & 1.50 & & & 1 & 0.80 \\
\hline \multirow[t]{6}{*}{$\beta=5$} & Two-Stage Method & 14.9469 & 1.5005 & 6.6599 & 1.7895 & 0.9232 & 0.7965 \\
\hline & & $(0.0106)$ & $(0.0035)$ & $(0.0132)$ & $(0.0093)$ & $(0.0039)$ & $(0.0040)$ \\
\hline & Traditional Analysis with Blocks & 15.4071 & 1.5013 & 2.2087 & - & - & 0.9992 \\
\hline & & $(0.0218)$ & $(0.0037)$ & $(0.0083)$ & - & - & $(0.0003)$ \\
\hline & Traditional Analysis & 16.8255 & 1.4925 & 0.7026 & - & - & 0.9610 \\
\hline & & $(0.0131)$ & $(0.0045)$ & $(0.0030)$ & - & - & $(0.0019)$ \\
\hline \multirow[t]{6}{*}{$\beta=3$} & Two-Stage Method & 14.8952 & 1.5013 & 4.0114 & 1.7720 & 0.9308 & 0.7948 \\
\hline & & $(0.0106)$ & $(0.0036)$ & $(0.0079)$ & $(0.0093)$ & $(0.0039)$ & $(0.0040)$ \\
\hline & Traditional Analysis with Blocks & 15.3321 & 1.5007 & 1.8347 & - & - & 0.9989 \\
\hline & & $(0.0214)$ & $(0.0037)$ & $(0.0057)$ & - & - & $(0.0003)$ \\
\hline & Traditional Analysis & 16.6926 & 1.4891 & 0.6724 & - & - & 0.9620 \\
\hline & & $(0.0130)$ & $(0.0045)$ & $(0.0027)$ & - & - & $(0.0019)$ \\
\hline \multirow[t]{6}{*}{$\beta=1$} & Two-Stage Method & 14.7430 & 1.4966 & 1.3334 & 1.7621 & 1.0295 & 0.7109 \\
\hline & & $(0.0108)$ & $(0.0040)$ & $(0.0026)$ & $(0.0096)$ & $(0.0043)$ & $(0.0045)$ \\
\hline & Traditional Analysis with Blocks & 15.1160 & 1.4965 & 0.9752 & - & - & 0.9950 \\
\hline & & $(0.0218)$ & $(0.0041)$ & $(0.0020)$ & - & - & $(0.0007)$ \\
\hline & Traditional Analysis & 16.3102 & 1.4955 & 0.5252 & - & - & 0.9216 \\
\hline & & $(0.0129)$ & $(0.0049)$ & $(0.0015)$ & - & - & $(0.0027)$ \\
\hline \multirow[t]{6}{*}{$\beta=0.5$} & Two-Stage Method & 14.4471 & 1.5113 & 0.6693 & 1.7318 & 1.3144 & 0.5278 \\
\hline & & $(0.01135)$ & $(0.0052)$ & $(0.0013)$ & $(0.0105)$ & $(0.0054)$ & $(0.0050)$ \\
\hline & Traditional Analysis with Blocks & 14.8205 & 1.5113 & 0.5561 & - & - & 0.9319 \\
\hline & & $(0.0226)$ & $(0.0052)$ & $(0.0010)$ & - & - & $(0.0025)$ \\
\hline & Traditional Analysis & 15.8625 & 1.5136 & 0.3830 & - & - & 0.8210 \\
\hline & & $(0.0131)$ & $(0.0058)$ & $(0.0009)$ & - & - & $(0.0038)$ \\
\hline
\end{tabular}

The estimates for $\gamma_{0}, \gamma_{1}, \beta, \sigma_{\rho}$ and $\sigma_{\omega}$ are the mean of the estimates for the 10,000 simulations. Simulation error is shown in parentheses. 
Table A.2: Simulation Results Power 0.80: $n=16, r=8$

\begin{tabular}{|c|c|c|c|c|c|c|c|}
\hline & \multirow[b]{2}{*}{ Actual } & $\gamma_{0}$ & $\gamma_{1}$ & $\beta$ & $\sigma_{\rho}$ & $\sigma_{\omega}$ & Power \\
\hline & & 15 & 1.50 & & 2 & 1 & 0.80 \\
\hline \multirow{6}{*}{$\beta=5$} & Two-Stage Method & 14.9710 & 1.5024 & 5.7038 & 1.7634 & 0.9168 & 0.7986 \\
\hline & & $(0.0107)$ & $(0.0036)$ & $(0.0071)$ & $(0.0091)$ & $(0.0038)$ & $(0.0040)$ \\
\hline & Traditional Analysis with Blocks & 15.4205 & 1.5025 & 2.1228 & - & - & 0.9999 \\
\hline & & $(0.0217)$ & $(0.0037)$ & $(0.0073)$ & - & - & $(0.0001)$ \\
\hline & Traditional Analysis & 16.8113 & 1.5046 & 0.7043 & - & - & 0.9855 \\
\hline & & $(0.0133)$ & $(0.0046)$ & $(0.0030)$ & - & - & $(0.0012)$ \\
\hline \multirow[t]{6}{*}{$\beta=3$} & Two-Stage Method & 14.9689 & 1.5037 & 3.4245 & 1.7873 & 0.9206 & 0.7937 \\
\hline & & $(0.0107)$ & $(0.0036)$ & $(0.0043)$ & (0.0093) & $(0.0039)$ & $(0.0040)$ \\
\hline & Traditional Analysis with Blocks & 15.3666 & 1.5040 & 1.7410 & - & - & 0.9997 \\
\hline & & $(0.0214)$ & $(0.0037)$ & $(0.0048)$ & - & - & $(0.0002)$ \\
\hline & Traditional Analysis & 16.7574 & 1.504 & 0.6565 & - & - & 0.9855 \\
\hline & & $(0.0130)$ & $(0.0045)$ & $(0.0025)$ & - & - & $(0.0012)$ \\
\hline \multirow[t]{6}{*}{$\beta=1$} & Two-Stage Method & 14.8564 & 1.500 & 1.1401 & 1.7829 & 0.9637 & 0.7651 \\
\hline & & $(0.0106)$ & $(0.0038)$ & $(0.0014)$ & $(0.0093)$ & $(0.0041)$ & $(0.0042)$ \\
\hline & Traditional Analysis with Blocks & 15.1307 & 1.4997 & 0.9014 & - & - & 0.9986 \\
\hline & & $(0.0216)$ & $(0.0038)$ & $(0.0014)$ & - & - & $(0.0004)$ \\
\hline & Traditional Analysis & 16.3528 & 1.5005 & 0.5065 & - & - & 0.9761 \\
\hline & & $(0.0127)$ & $(0.0045)$ & $(0.0014)$ & - & - & $(0.0015)$ \\
\hline \multirow[t]{6}{*}{$\beta=0.5$} & Two-Stage Method & 14.7480 & 1.5041 & 0.5699 & 1.7569 & 1.1214 & 0.6522 \\
\hline & & $(0.0109)$ & $(0.0043)$ & $(0.0007)$ & $(0.0099)$ & $(0.0046)$ & $(0.0048)$ \\
\hline & Traditional Analysis with Blocks & 15.0076 & 1.5041 & 0.5089 & - & - & 0.9865 \\
\hline & & $(0.0219)$ & $(0.0043)$ & $(0.0006)$ & - & - & $(0.0012)$ \\
\hline & Traditional Analysis & 15.9926 & 1.5071 & 0.3665 & - & - & 0.9403 \\
\hline & & $(0.0126)$ & $(0.0049)$ & $(0.0007)$ & - & - & $(0.0024)$ \\
\hline
\end{tabular}

The estimates for $\gamma_{0}, \gamma_{1}, \beta, \sigma_{\rho}$ and $\sigma_{\omega}$ are the mean of the estimates for the 10,000 simulations. Simulation error is shown in parentheses. 
Jennifer L. K. Kensler Appendix A. Two-Stage Simulation Study Tables and Figures 137

Table A.3: Simulation Results Power 0.80: $n=16, r=12$

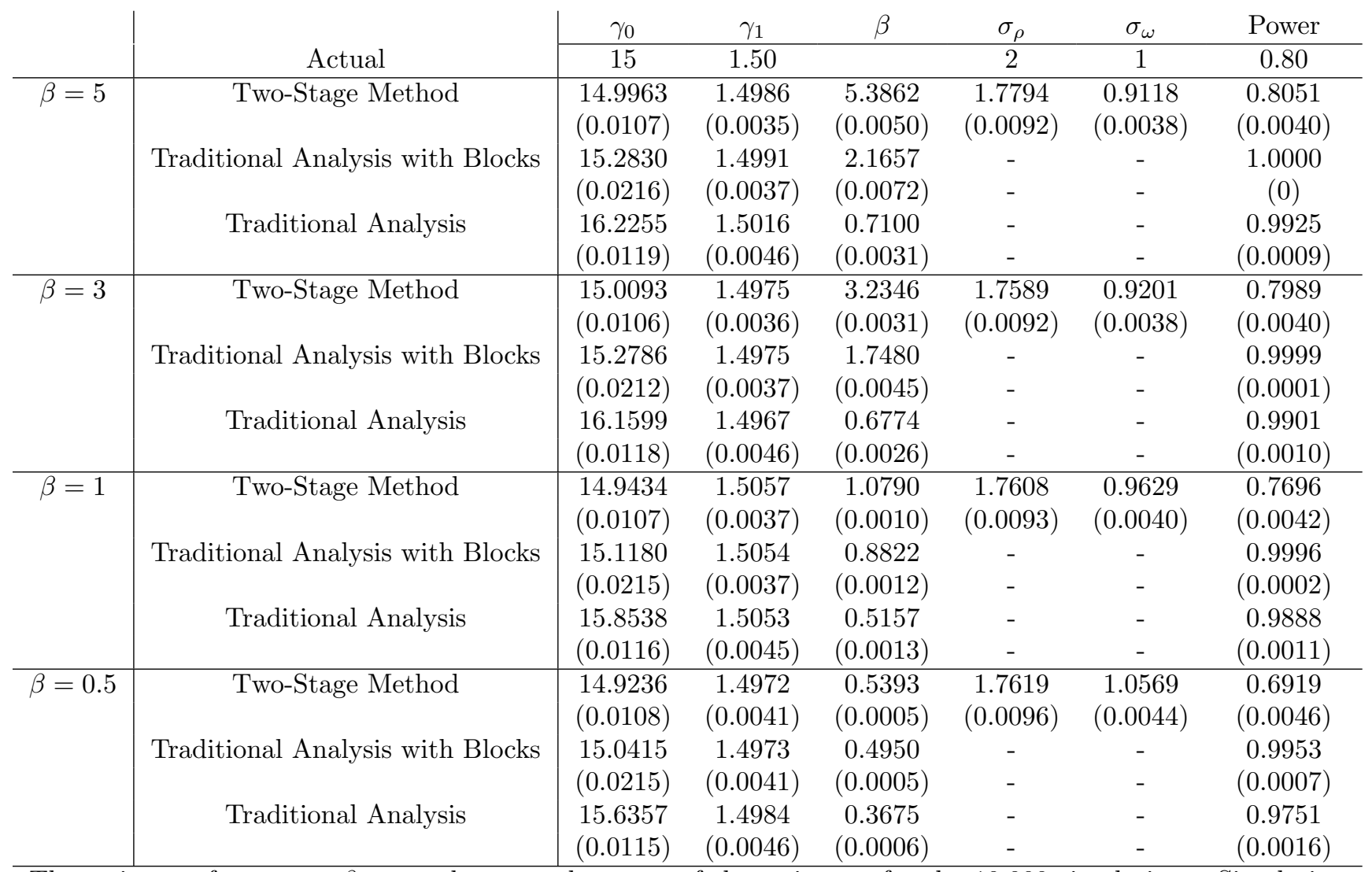

The estimates for $\gamma_{0}, \gamma_{1}, \beta, \sigma_{\rho}$ and $\sigma_{\omega}$ are the mean of the estimates for the 10,000 simulations. Simulation error is shown in parentheses.

\section{A.2 Figures for the Two-Stage Simulation Study}

Figure A.1 compares the bias of the Weibull shape parameter estimates for the three methods by plotting the ratio of $\hat{\beta}$ to $\beta$. 
Jennifer L. K. Kensler Appendix A. Two-Stage Simulation Study Tables and Figures 138

Figure A.1: Ratio of $\hat{\beta}$ to $\beta$

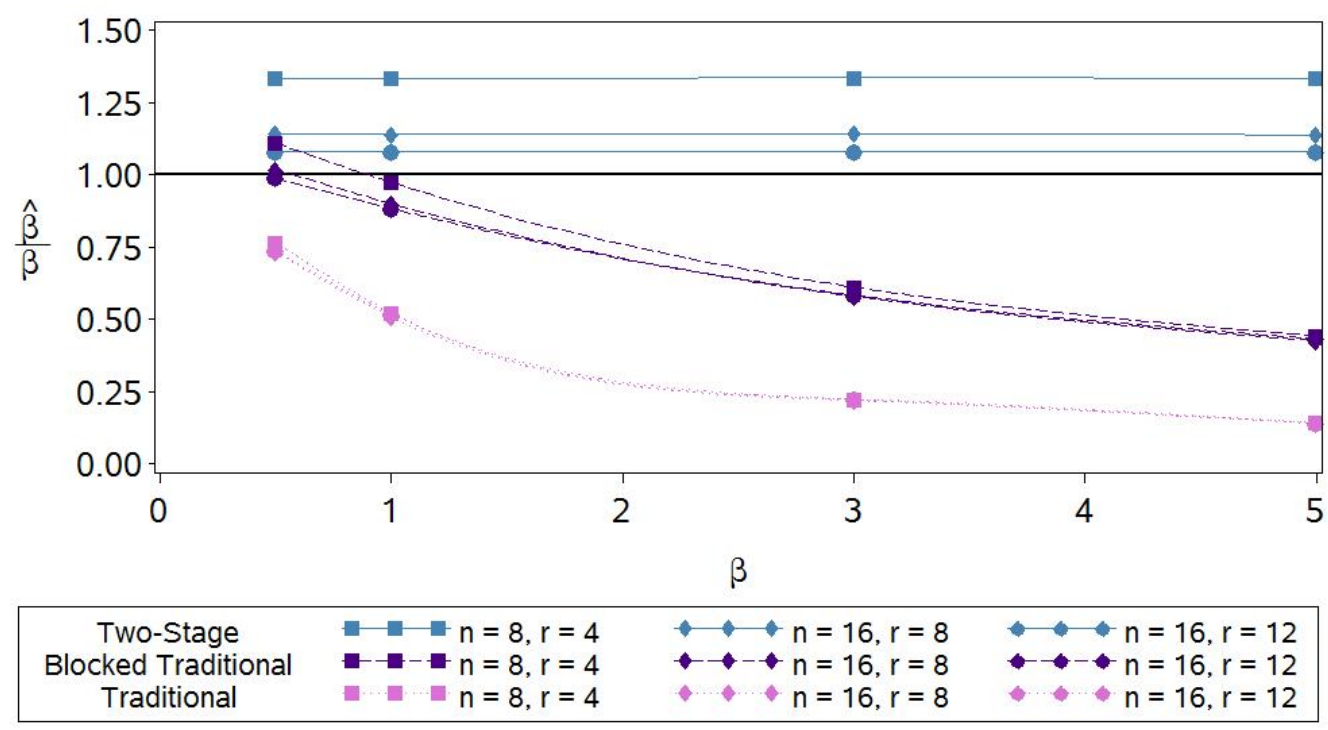

Figure A.2 compares the estimates of $\gamma_{0}$ for the three methods to its true value of 15 .

Figure A.2: $\hat{\gamma}_{0}$

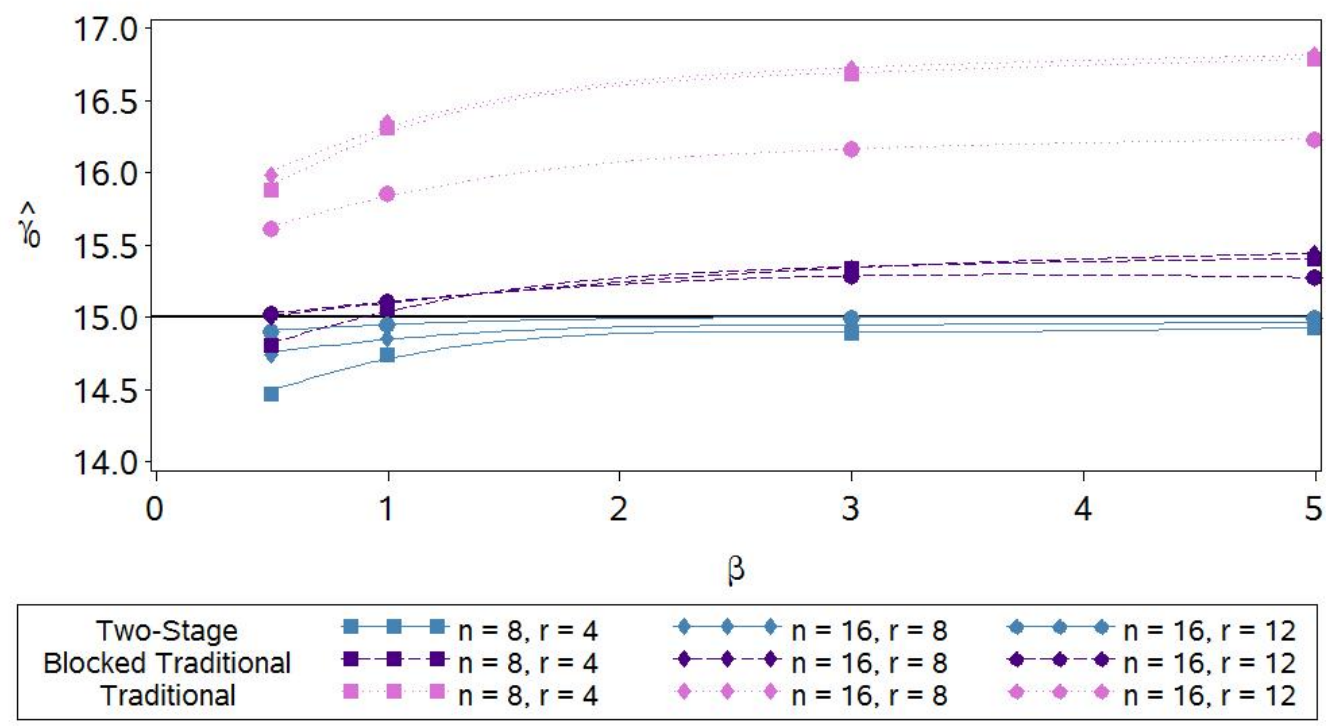

Figure A.3 compares the estimates of $\gamma_{1}$ for the three methods when the true value is 0 . 
Jennifer L. K. Kensler Appendix A. Two-Stage Simulation Study Tables and Figures 139

Figure A.3: $\hat{\gamma}_{1}$

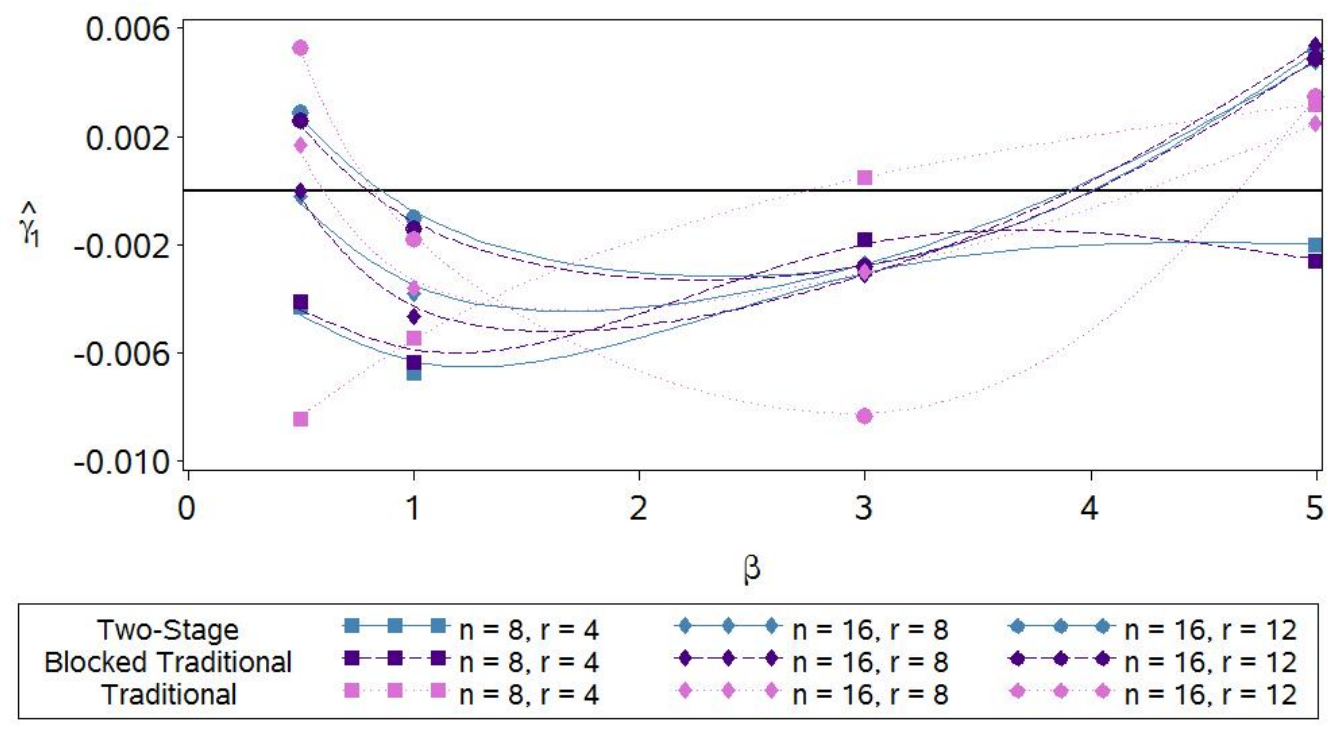

Figure A.4 compares the estimates of $\gamma_{1}$ for the three methods when the true value is 1.01.

Figure A.4: $\hat{\gamma}_{1}$ for Nominal Power of 0.50

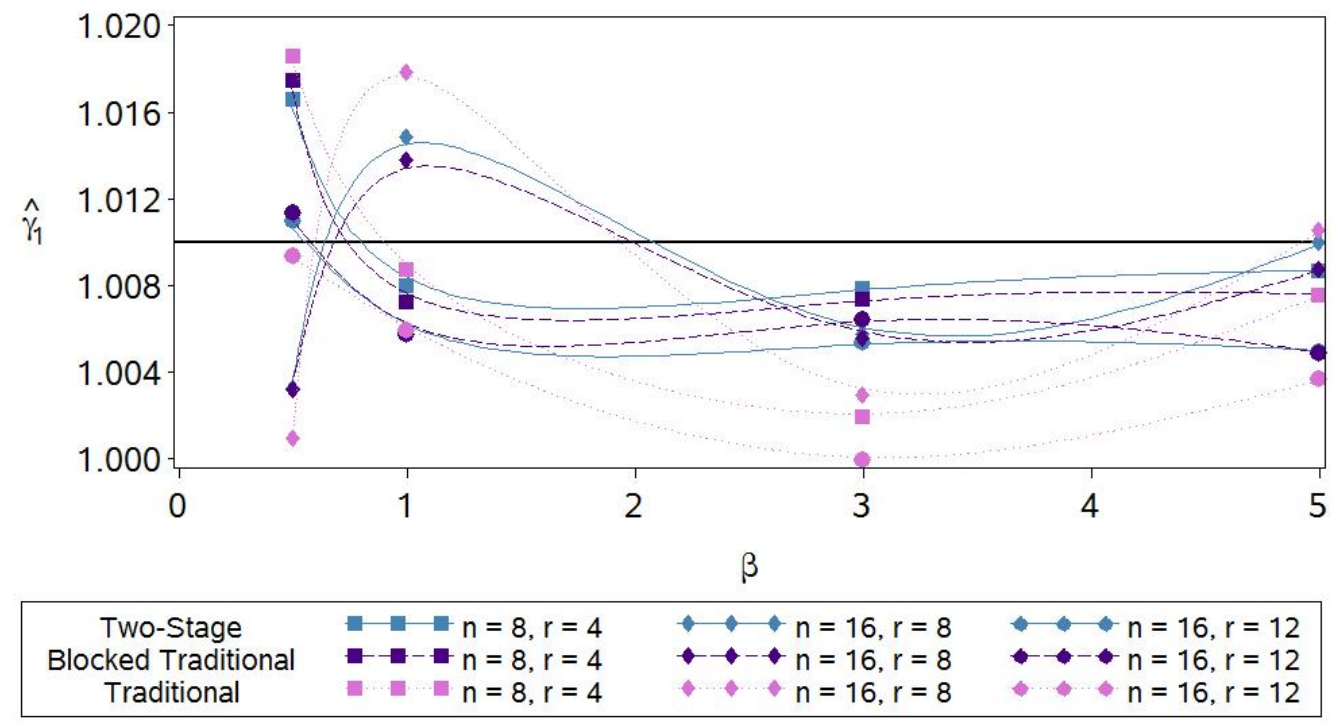

Figure A.5 compares the estimates of $\gamma_{1}$ for the three methods when the true value is 1.5 . 
Jennifer L. K. Kensler Appendix A. Two-Stage Simulation Study Tables and Figures 140

Figure A.5: $\hat{\gamma}_{1}$ for Nominal Power of 0.80

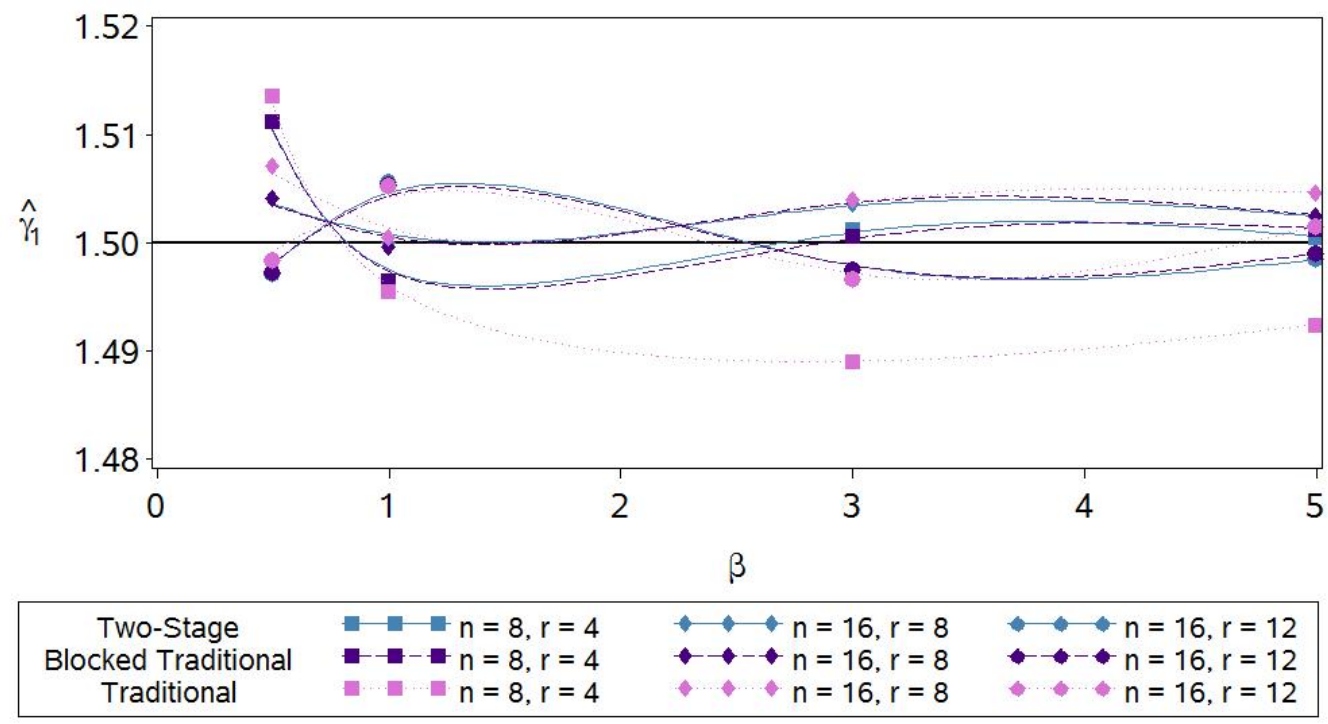

Figure A.6 compares the estimates of $\gamma_{1}$ for the three methods when the true value is 1.77 .

Figure A.6: $\hat{\gamma}_{1}$ for Nominal Power of 0.90

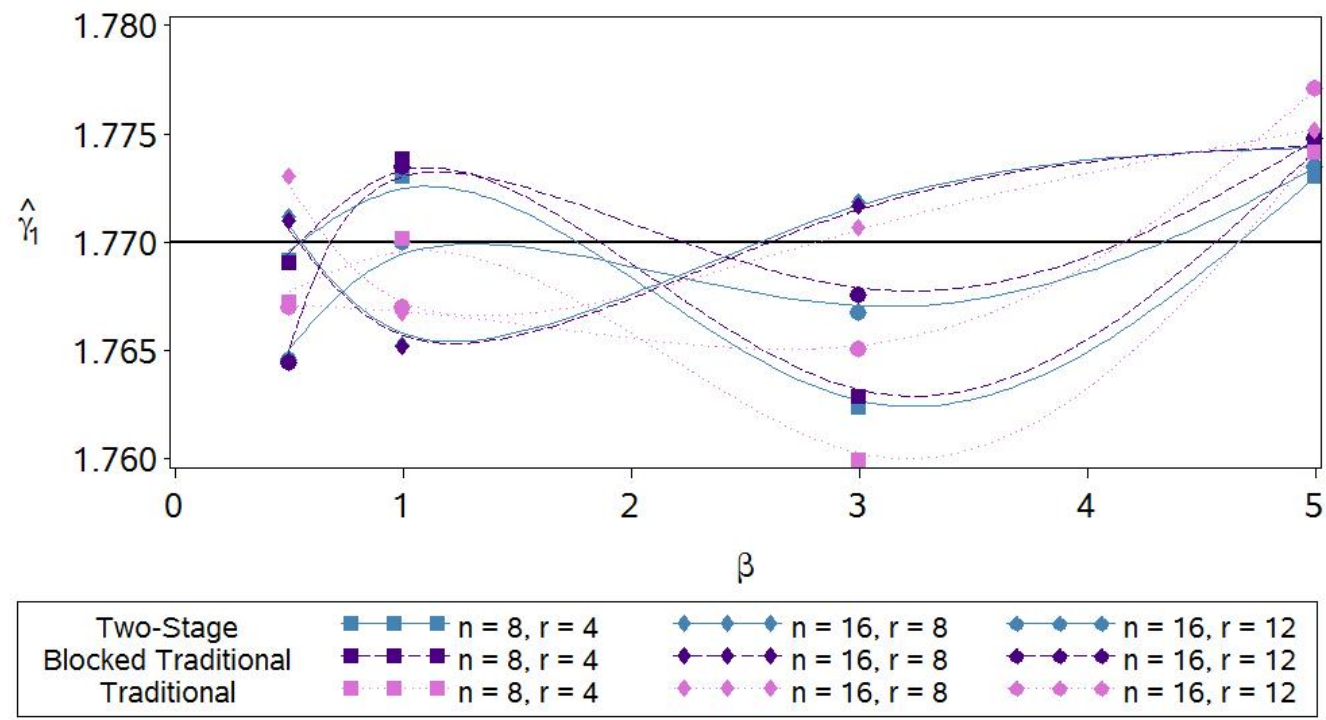

Figure A.7 compares the estimates of $\sigma_{\rho}$ for the two-stage method to the actual value of 2 . 
Jennifer L. K. Kensler Appendix A. Two-Stage Simulation Study Tables and Figures 141

Figure A.7: $\hat{\sigma}_{\rho}$

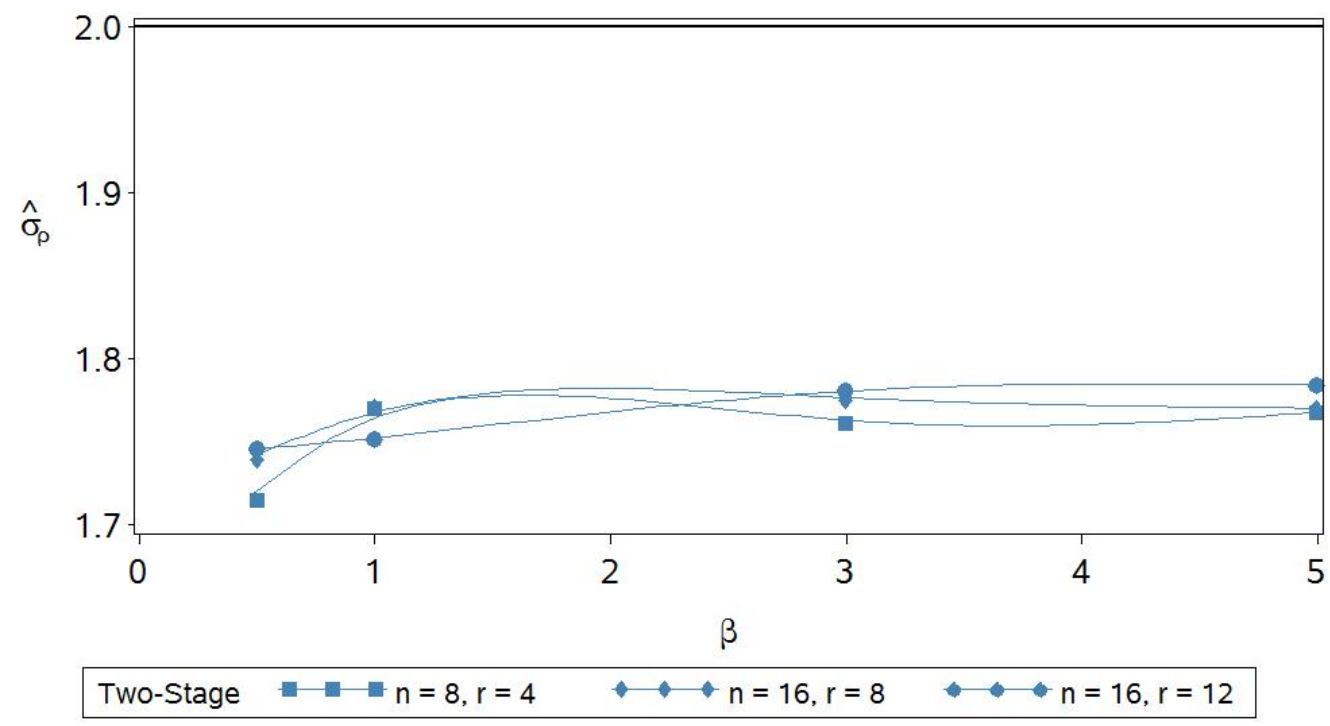

Figure A.8 compares the estimates of $\sigma_{\omega}$ for the two-stage method to the actual value of 1 .

Figure A.8: $\hat{\sigma}_{\omega}$

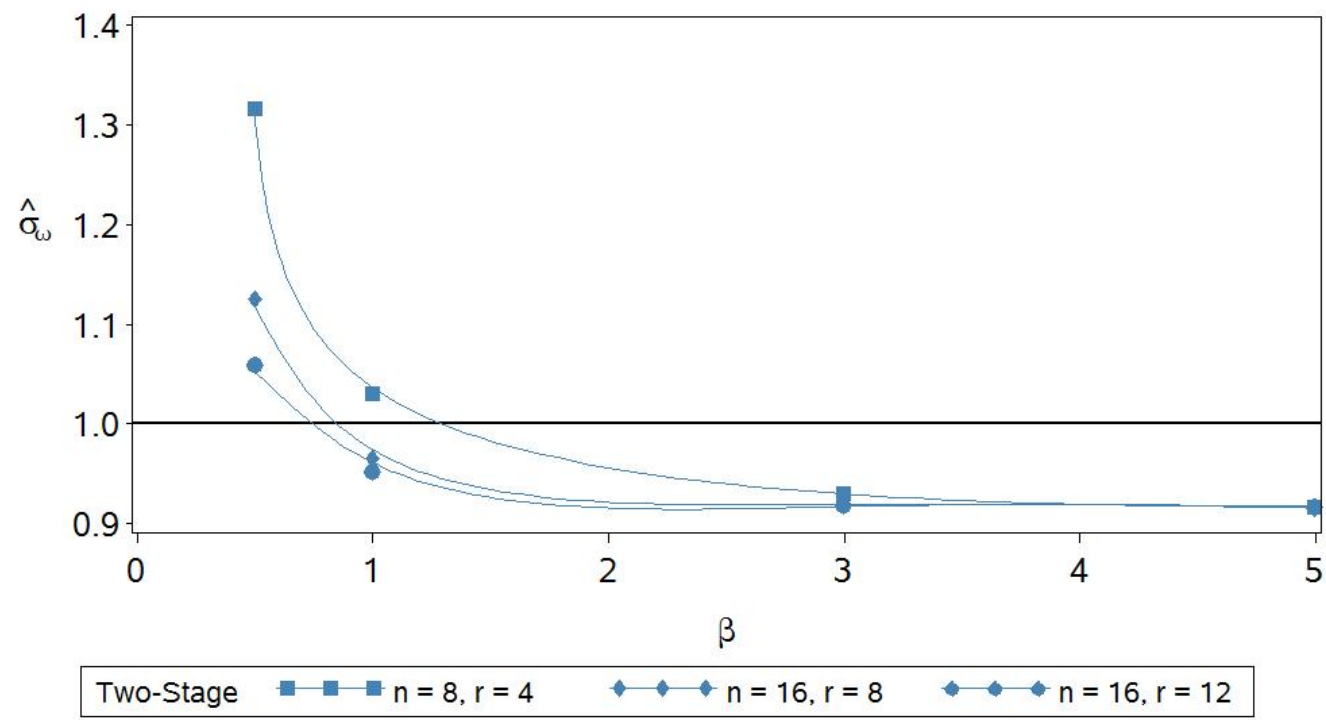

Figure A.9 depicts the empirical error under a nominal type I error rate of 0.05 . 
Jennifer L. K. Kensler Appendix A. Two-Stage Simulation Study Tables and Figures 142

Figure A.9: Empirical Error Rate

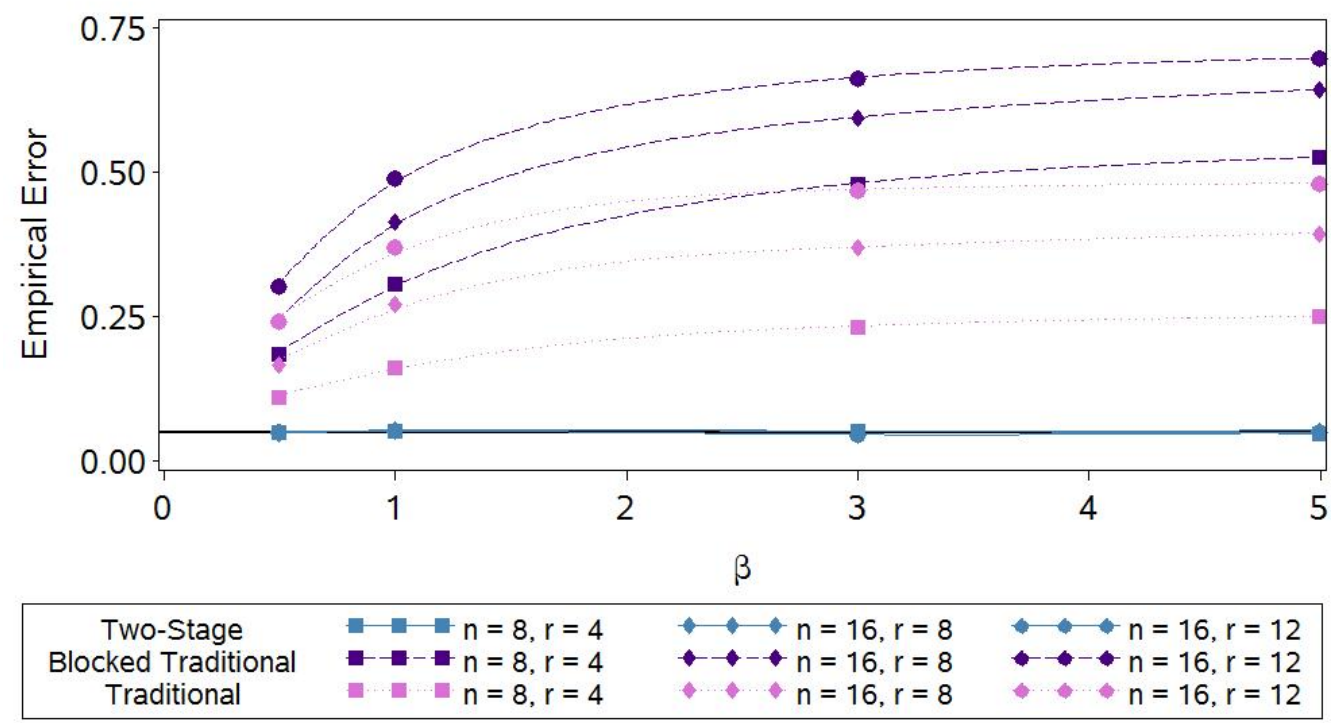

Figures A.10 - A.12 show the empirical powers for nominal powers of 0.50, 0.80, and 0.90 respectively.

Figure A.10: Nominal Power of 0.50

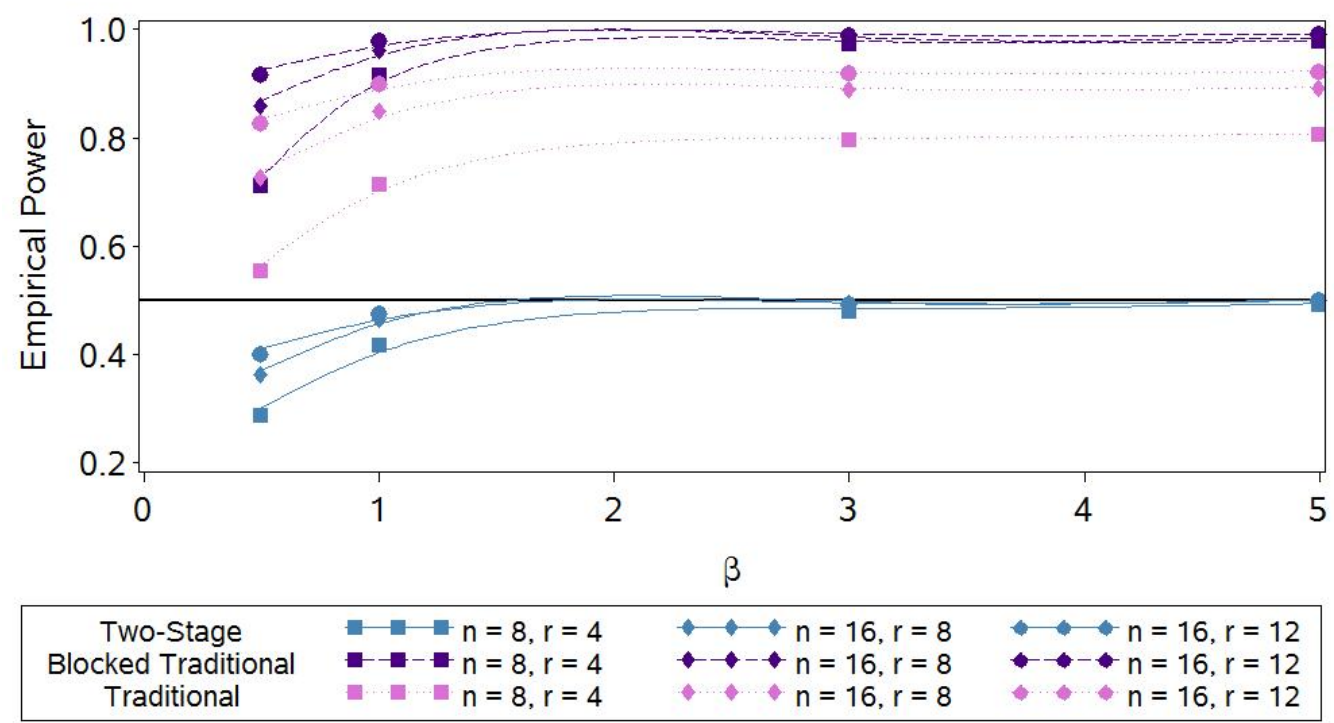


Jennifer L. K. Kensler Appendix A. Two-Stage Simulation Study Tables and Figures 143

Figure A.11: Nominal Power of 0.80

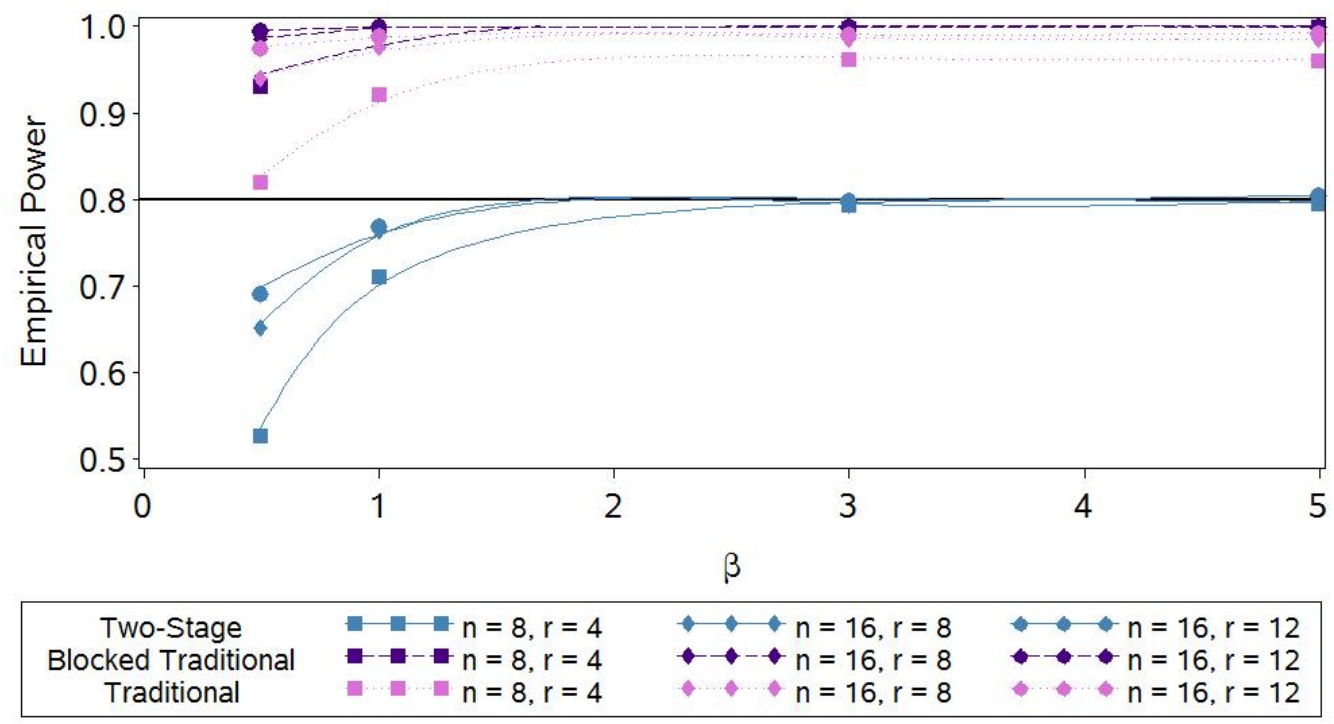

Figure A.12: Nominal Power of 0.90

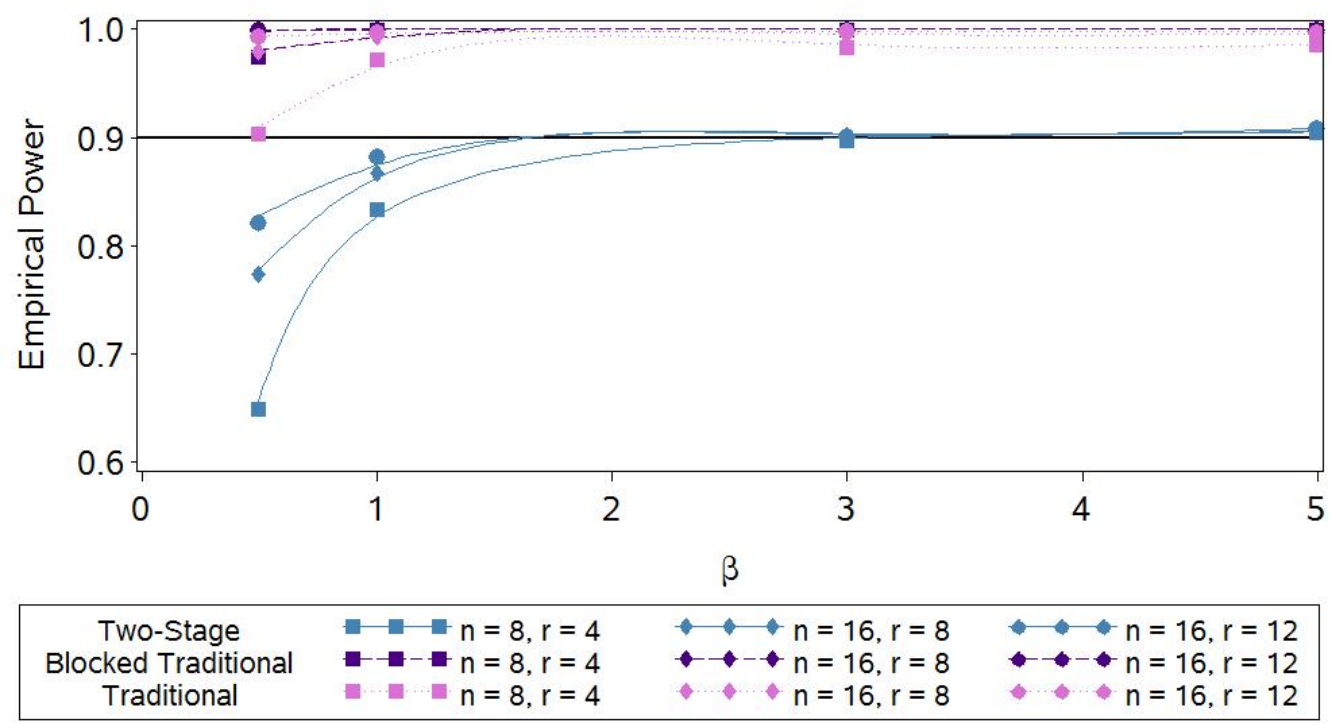




\section{Appendix B}

\section{Derivation of Variance-Covariance}

\section{Matrix}

Define the following:

$$
\begin{gathered}
P_{1}=\prod_{k(i j)=1}^{n}\left(g\left(t_{k(i j)} \mid \sqrt{2} \sigma_{\rho} q_{2, k 2}, \sqrt{2} \sigma_{\omega} q_{1, k 1}\right)\right) \\
P_{2}=\prod_{i=1}^{m}\left(\sum_{k 1=1}^{n_{k 1}} w_{1, k 1} P_{1}\right) \\
Q_{a, b, c, d}=\sum_{i=1}^{m} x_{i}^{a} \frac{\sum_{r=1}^{n_{k 1}} w_{1, k 1} q_{1, k 1}^{b} P_{1}\left(S_{0,0,1}-S_{1,0,0}\right)^{c}\left(S_{1,0,0}+S_{1,1,0}-S_{0,1,1}\right)^{d}}{\sum_{k 1=1}^{n_{k 1}} w_{1, k 1} P_{1}} .
\end{gathered}
$$


The first order partial derivatives of the approximate log-likelihood are

$$
\begin{gathered}
\frac{\partial \mathcal{L}\left(\boldsymbol{\gamma}, \beta, \sigma_{\rho}, \sigma_{\omega} \mid \text { Data }\right)}{\partial \gamma_{0}} \approx \beta \sum_{j=1}^{b} \frac{\sum_{k 2=1}^{n_{k 2}} w_{2, k 2} P_{2} Q_{0,0,1,0}}{\sum_{k 2=1}^{n_{k 2}} w_{2, k 2} P_{2}} \\
\frac{\partial \mathcal{L}\left(\boldsymbol{\gamma}, \beta, \sigma_{\rho}, \sigma_{\omega} \mid \text { Data }\right)}{\partial \gamma_{1}} \approx \beta \sum_{j=1}^{b} \frac{\sum_{k 2=1}^{n_{k 2}} w_{2, k 2} P_{2} Q_{1,0,1,0}}{\sum_{k 2=1}^{n_{k 2}} w_{2, k 2} P_{2}} \\
\frac{\partial \mathcal{L}\left(\boldsymbol{\gamma}, \beta, \sigma_{\rho}, \sigma_{\omega} \mid D a t a\right)}{\partial \beta} \approx \frac{1}{\beta} \sum_{j=1}^{b} \frac{\sum_{k 2=1}^{n_{k 2}} w_{2, k 2} P_{2} Q_{0,0,0,1}}{\sum_{k 2=1}^{n_{k 2}} w_{2, k 2} P_{2}} \\
\frac{\partial \mathcal{L}\left(\boldsymbol{\gamma}, \beta, \sigma_{\rho}, \sigma_{\omega} \mid \text { Data }\right)}{\partial \sigma_{\rho}} \approx \sqrt{2} \beta \sum_{j=1}^{b} \frac{\sum_{k 2=1}^{n_{k 2}} w_{2, k 2} q_{2, k 2} P_{2} Q_{0,0,1,0}}{\sum_{k 2=1}^{n_{k 2}} w_{2, k 2} P_{2}} \\
\frac{\partial \mathcal{L}\left(\boldsymbol{\gamma}, \beta, \sigma_{\rho}, \sigma_{\omega} \mid \text { Data }\right)}{\partial \sigma_{\omega}} \approx \sqrt{2} \beta \sum_{j=1}^{b} \frac{\sum_{k 2=1}^{n_{k 2}} w_{2, k 2} P_{2} Q_{0,1,1,0}}{\sum_{k 2=1}^{n_{k} 2} w_{2, k 2} P_{2}} .
\end{gathered}
$$

Define:

$$
D_{11}=\sum_{i=1}^{m} \frac{\left[\sum_{k 1=1}^{n_{k 1}} w_{1, k 1} P_{1}\right] \times A-B \times C}{\left[\sum_{k 1=1}^{n_{k 1}} w_{1, k 1} P_{1}\right]^{2}}
$$


where

$$
\begin{aligned}
& A=\sum_{k 1=1}^{n_{k 1}} w_{1, k 1} P_{1}\left[\left(S_{0,0,1}-S_{1,0,0}\right)^{2}-S_{0,0,1}\right] \\
& B=\sum_{k 1=1}^{n_{k 1}} w_{1, k 1} P_{1}\left(S_{0,0,1}-S_{1,0,0}\right) \\
& C=\sum_{k 1=1}^{n_{k 1}} w_{1, k 1} P_{1}\left(S_{0,0,1}-S_{1,0,0}\right)
\end{aligned}
$$

$$
D_{12}=\sum_{i=1}^{m} x_{i} \frac{\left[\sum_{k 1=1}^{n_{k 1}} w_{1, k 1} P_{1}\right] \times A-B \times C}{\left[\sum_{k 1=1}^{n_{k 1}} w_{1, k 1} P_{1}\right]^{2}}
$$

where

$$
\begin{aligned}
& A=\sum_{k 1=1}^{n_{k 1}} w_{1, k 1} P_{1}\left[\left(S_{0,0,1}-S_{1,0,0}\right)^{2}-S_{0,0,1}\right] \\
& B=\sum_{k 1=1}^{n_{k 1}} w_{1, k 1} P_{1}\left(S_{0,0,1}-S_{1,0,0}\right) \\
& C=\sum_{k 1=1}^{n_{k 1}} w_{1, k 1} P_{1}\left(S_{0,0,1}-S_{1,0,0}\right)
\end{aligned}
$$

$$
D_{13}=\sum_{i=1}^{m} \frac{\left[\sum_{k 1=1}^{n_{k 1}} w_{1, k 1} P_{1}\right] \times A-B \times C}{\left[\sum_{k 1=1}^{n_{k 1}} w_{1, k 1} P_{1}\right]^{2}}
$$


where

$$
\begin{aligned}
& A=\sum_{k 1=1}^{n_{k 1}} w_{1, k 1} P_{1}\left[\left(S_{1,0,0}+S_{1,1,0}-S_{0,1,1}\right)\left(S_{0,0,1}-S_{1,0,0}\right)+S_{0,1,1}\right] \\
& B=\sum_{k 1=1}^{n_{k 1}} w_{1, k 1} P_{1}\left(S_{0,0,1}-S_{1,0,0}\right) \\
& C=\sum_{k 1=1}^{n_{k 1}} w_{1, k 1} P_{1}\left(S_{1,0,0}+S_{1,1,0}-S_{0,1,1}\right)
\end{aligned}
$$

$$
D_{15}=\sum_{i=1}^{m} \frac{\left[\sum_{k 1=1}^{n_{k 1}} w_{1, k 1} P_{1}\right] \times A-B \times C}{\left[\sum_{k 1=1}^{n_{k 1}} w_{1, k 1} P_{1}\right]^{2}}
$$

where

$$
\begin{aligned}
& A=\sum_{k 1=1}^{n_{k 1}} w_{1, k 1} q_{1, k 1} P_{1}\left[\left(S_{0,0,1}-S_{1,0,0}\right)^{2}-S_{0,0,1}\right] \\
& B=\sum_{k 1=1}^{n_{k 1}} w_{1, k 1} P_{1}\left(S_{0,0,1}-S_{1,0,0}\right) \\
& C=\sum_{k 1=1}^{n_{k 1}} w_{1, k 1} q_{1, k 1} P_{1}\left(S_{0,0,1}-S_{1,0,0}\right)
\end{aligned}
$$

$$
D_{22}=\sum_{i=1}^{m} x_{i}^{2} \frac{\left[\sum_{k 1=1}^{n_{k 1}} w_{1, k 1} P_{1}\right] \times A-B \times C}{\left[\sum_{k 1=1}^{n_{k 1}} w_{1, k 1} P_{1}\right]^{2}}
$$


where

$$
\begin{aligned}
& A=\sum_{k 1=1}^{n_{k 1}} w_{1, k 1} P_{1}\left[\left(S_{0,0,1}-S_{1,0,0}\right)^{2}-S_{0,0,1}\right] \\
& B=\sum_{k 1=1}^{n_{k 1}} w_{1, k 1} P_{1}\left(S_{0,0,1}-S_{1,0,0}\right) \\
& C=\sum_{k 1=1}^{n_{k 1}} w_{1, k 1} P_{1}\left(S_{0,0,1}-S_{1,0,0}\right)
\end{aligned}
$$

$$
D_{23}=\sum_{i=1}^{m} x_{i} \frac{\left[\sum_{k 1=1}^{n_{k 1}} w_{1, k 1} P_{1}\right] \times A-B \times C}{\left[\sum_{k 1=1}^{n_{k 1}} w_{1, k 1} P_{1}\right]^{2}}
$$

where

$$
\begin{aligned}
& A=\sum_{k 1=1}^{n_{k 1}} w_{1, k 1} P_{1}\left[\left(S_{1,0,0}+S_{1,1,0}-S_{0,1,1}\right)\left(S_{0,0,1}-S_{1,0,0}\right)+S_{0,1,1}\right] \\
& B=\sum_{k 1=1}^{n_{k 1}} w_{1, k 1} P_{1}\left(S_{0,0,1}-S_{1,0,0}\right) \\
& C=\sum_{k 1=1}^{n_{k 1}} w_{1, k 1} P_{1}\left(S_{1,0,0}+S_{1,1,0}-S_{0,1,1}\right)
\end{aligned}
$$

$$
D_{25}=\sum_{i=1}^{m} x_{i} \frac{\left[\sum_{k 1=1}^{n_{k 1}} w_{1, k 1} P_{1}\right] \times A-B \times C}{\left[\sum_{k 1=1}^{n_{k 1}} w_{1, k 1} P_{1}\right]^{2}}
$$


where

$$
\begin{aligned}
& A=\sum_{k 1=1}^{n_{k 1}} w_{1, k 1} q_{1, k 1} P_{1}\left[\left(S_{0,0,1}-S_{1,0,0}\right)^{2}-S_{0,0,1}\right] \\
& B=\sum_{k 1=1}^{n_{k 1}} w_{1, k 1} P_{1}\left(S_{0,0,1}-S_{1,0,0}\right) \\
& C=\sum_{k 1=1}^{n_{k 1}} w_{1, k 1} q_{1, k 1} P_{1}\left(S_{0,0,1}-S_{1,0,0}\right)
\end{aligned}
$$

$$
D_{33}=\sum_{i=1}^{m} \frac{\left[\sum_{k 1=1}^{n_{k 1}} w_{1, k 1} P_{1}\right] \times A-B \times C}{\left[\sum_{k 1=1}^{n_{k 1}} w_{1, k 1} P_{1}\right]^{2}}
$$

where

$$
\begin{aligned}
& A=\sum_{k 1=1}^{n_{k 1}} w_{1, k 1} P_{1}\left[\left(S_{1,0,0}+S_{1,1,0}-S_{0,1,1}\right)^{2}+S_{1,1,0}-S_{0,1,1}-S_{0,2,1}\right] \\
& B=\sum_{k 1=1}^{n_{k 1}} w_{1, k 1} P_{1}\left(S_{1,0,0}+S_{1,1,0}-S_{0,1,1}\right) \\
& C=\sum_{k 1=1}^{n_{k 1}} w_{1, k 1} P_{1}\left(S_{1,0,0}+S_{1,1,0}-S_{0,1,1}\right)
\end{aligned}
$$

$$
D_{34}=\sum_{i=1}^{m} \frac{\left[\sum_{k 1=1}^{n_{k 1}} w_{1, k 1} P_{1}\right] \times A-B \times C}{\left[\sum_{k 1=1}^{n_{k 1}} w_{1, k 1} P_{1}\right]^{2}}
$$


where

$$
\begin{aligned}
& A=\sum_{k 1=1}^{n_{k 1}} w_{1, k 1} P_{1}\left[\left(S_{0,0,1}-S_{1,0,0}\right)\left(S_{1,0,0}+S_{1,1,0}-S_{0,1,1}\right)-S_{1,0,0}+S_{0,0,1}+S_{0,1,1}\right] \\
& B=\sum_{k 1=1}^{n_{k 1}} w_{1, k 1} P_{1}\left(S_{1,0,0}+S_{1,1,0}-S_{0,1,1}\right) \\
& C=\sum_{k 1=1}^{n_{k 1}} w_{1, k 1} P_{1}\left(S_{0,0,1}-S_{1,0,0}\right) \\
& D_{35}=\sum_{i=1}^{m} \frac{\left[\sum_{k 1=1}^{n_{k 1}} w_{1, k 1} P_{1}\right] \times A-B \times C}{\left[\sum_{k 1=1}^{n_{k 1}} w_{1, k 1} P_{1}\right]^{2}}
\end{aligned}
$$

where

$$
\begin{aligned}
& A=\sum_{k 1=1}^{n_{k 1}} w_{1, k 1} q_{1, k 1} P_{1}\left[\left(S_{0,0,1}-S_{1,0,0}\right)\left(S_{1,0,0}+S_{1,1,0}-S_{0,1,1}\right)-S_{1,0,0}+S_{0,0,1}+S_{0,1,1}\right] \\
& B=\sum_{k 1=1}^{n_{k 1}} w_{1, k 1} P_{1}\left(S_{1,0,0}+S_{1,1,0}-S_{0,1,1}\right) \\
& C=\sum_{k 1=1}^{n_{k 1}} w_{1, k 1} q_{1, k 1} P_{1}\left(S_{0,0,1}-S_{1,0,0}\right)
\end{aligned}
$$

$$
D_{55}=\sum_{i=1}^{m} \frac{\left[\sum_{k 1=1}^{n_{k 1}} w_{1, k 1} P_{1}\right] \times A-B \times C}{\left[\sum_{k 1=1}^{n_{k 1}} w_{1, k 1} P_{1}\right]^{2}}
$$


where

$$
\begin{aligned}
& A=\sum_{k 1=1}^{n_{k 1}} w_{1, k 1} q_{1, k 1}^{2} P_{1}\left[\left(S_{0,0,1}-S_{1,0,0}\right)^{2}-S_{0,0,1}\right] \\
& B=\sum_{k 1=1}^{n_{k 1}} w_{1, k 1} q_{1, k 1} P_{1}\left(S_{0,0,1}-S_{1,0,0}\right) \\
& C=\sum_{k 1=1}^{n_{k 1}} w_{1, k 1} q_{1, k 1} P_{1}\left(S_{0,0,1}-S_{1,0,0}\right) .
\end{aligned}
$$

The second order partial derivatives of the approximate log-likelihood are

$$
\frac{\partial^{2} \mathcal{L}\left(\boldsymbol{\gamma}, \beta, \sigma_{\rho}, \sigma_{\omega} \mid D a t a\right)}{\partial \gamma_{0}^{2}} \approx \beta^{2} \sum_{j=1}^{b} \frac{\left[\sum_{k 2=1}^{n_{k 2}} w_{2, k 2} P_{2}\right] \times D-E \times F}{\left[\sum_{k 2=1}^{n_{k 2}} w_{2, k 2} P_{2}\right]^{2}}
$$

where

$$
\begin{aligned}
& D=\sum_{k 2=1}^{n_{k 2}} w_{2, k 2} P_{2}\left[Q_{0,0,1,0}^{2}+D_{11}\right] \\
& E=\sum_{k 2=1}^{n_{k 2}} w_{2, k 2} P_{2} Q_{0,0,1,0} \\
& F=\sum_{k 2=1}^{n_{k 2}} w_{2, k 2} P_{2} Q_{0,0,1,0}
\end{aligned}
$$


Jennifer L. K. Kensler Appendix B. Derivation of Variance-Covariance Matrix

$$
\frac{\partial^{2} \mathcal{L}\left(\boldsymbol{\gamma}, \beta, \sigma_{\rho}, \sigma_{\omega} \mid \text { Data }\right)}{\partial \gamma_{0} \partial \gamma_{1}} \approx \beta^{2} \sum_{j=1}^{b} \frac{\left[\sum_{k 2=1}^{n_{k 2}} w_{2, k 2} P_{2}\right] \times D-E \times F}{\left[\sum_{k 2=1}^{n_{k 2}} w_{2, k 2} P_{2}\right]^{2}}
$$

where

$$
\begin{aligned}
& D=\sum_{k 2=1}^{n_{k 2}} w_{2, k 2} P_{2}\left[Q_{1,0,1,0} Q_{0,0,1,0}+D_{12}\right] \\
& E=\sum_{k 2=1}^{n_{k 2}} w_{2, k 2} P_{2} Q_{0,0,1,0} \\
& F=\sum_{k 2=1}^{n_{k 2}} w_{2, k 2} P_{2} Q_{1,0,1,0}
\end{aligned}
$$

$$
\frac{\partial^{2} \mathcal{L}\left(\gamma, \beta, \sigma_{\rho}, \sigma_{\omega} \mid \text { Data }\right)}{\partial \gamma_{0} \partial \beta} \approx \sum_{j=1}^{b} \frac{\left[\sum_{k 2=1}^{n_{k 2}} w_{2, k 2} P_{2}\right] \times D-E \times F}{\left[\sum_{k 2=1}^{n_{k 2}} w_{2, k 2} P_{2}\right]^{2}}
$$

where

$$
\begin{aligned}
& D=\sum_{k 2=1}^{n_{k 2}} w_{2, k 2} P_{2}\left[Q_{0,0,1,0}+Q_{0,0,0,1} Q_{0,0,1,0}+D_{13}\right] \\
& E=\sum_{k 2=1}^{n_{k 2}} w_{2, k 2} P_{2} Q_{0,0,1,0} \\
& F=\sum_{k 2=1}^{n_{k 2}} w_{2, k 2} P_{2} Q_{0,0,0,1}
\end{aligned}
$$


Jennifer L. K. Kensler Appendix B. Derivation of Variance-Covariance Matrix

153

$$
\frac{\partial^{2} \mathcal{L}\left(\boldsymbol{\gamma}, \beta, \sigma_{\rho}, \sigma_{\omega} \mid \text { Data }\right)}{\partial \gamma_{0} \partial \sigma_{\rho}} \approx \sqrt{2} \beta^{2} \sum_{j=1}^{b} \frac{\left[\sum_{k 2=1}^{n_{k 2}} w_{2, k 2} P_{2}\right] \times D-E \times F}{\left[\sum_{k 2=1}^{n_{k 2}} w_{2, k 2} P_{2}\right]^{2}}
$$

where

$$
\begin{aligned}
& D=\sum_{k 2=1}^{n_{k 2}} w_{2, k 2} q_{2, k 2} P_{2}\left[Q_{0,0,1,0}^{2}+D_{11}\right] \\
& E=\sum_{k 2=1}^{n_{k 2}} w_{2, k 2} P_{2} Q_{0,0,1,0} \\
& F=\sum_{k 2=1}^{n_{k 2}} w_{2, k 2} q_{2, k 2} P_{2} Q_{0,0,1,0}
\end{aligned}
$$

$$
\frac{\partial^{2} \mathcal{L}\left(\gamma, \beta, \sigma_{\rho}, \sigma_{\omega} \mid \text { Data }\right)}{\partial \gamma_{0} \partial \sigma_{\omega}} \approx \sqrt{2} \beta^{2} \sum_{j=1}^{b} \frac{\left[\sum_{k 2=1}^{n_{k 2}} w_{2, k 2} P_{2}\right] \times D-E \times F}{\left[\sum_{k 2=1}^{n_{k 2}} w_{2, k 2} P_{2}\right]^{2}}
$$

where

$$
\begin{aligned}
& D=\sum_{k 2=1}^{n_{k 2}} w_{2, k 2} P_{2}\left[Q_{0,1,1,0} Q_{0,0,1,0}+D_{15}\right] \\
& E=\sum_{k 2=1}^{n_{k 2}} w_{2, k 2} P_{2} Q_{0,0,1,0} \\
& F=\sum_{k 2=1}^{n_{k 2}} w_{2, k 2} P_{2} Q_{0,1,1,0}
\end{aligned}
$$


Jennifer L. K. Kensler Appendix B. Derivation of Variance-Covariance Matrix

$$
\frac{\partial^{2} \mathcal{L}\left(\boldsymbol{\gamma}, \beta, \sigma_{\rho}, \sigma_{\omega} \mid \text { Data }\right)}{\partial \gamma_{1}^{2}} \approx \beta^{2} \sum_{j=1}^{b} \frac{\left[\sum_{k 2=1}^{n_{k 2}} w_{2, k 2} P_{2}\right] \times D-E \times F}{\left[\sum_{k 2=1}^{n_{k 2}} w_{2, k 2} P_{2}\right]^{2}}
$$

where

$$
\begin{aligned}
& D=\sum_{k 2=1}^{n_{k 2}} w_{2, k 2} P_{2}\left[Q_{1,0,1,0}^{2}+D_{22}\right] \\
& E=\sum_{k 2=1}^{n_{k 2}} w_{2, k 2} P_{2} Q_{1,0,1,0} \\
& F=\sum_{k 2=1}^{n_{k 2}} w_{2, k 2} P_{2} Q_{1,0,1,0}
\end{aligned}
$$

$$
\frac{\partial^{2} \mathcal{L}\left(\gamma, \beta, \sigma_{\rho}, \sigma_{\omega} \mid \text { Data }\right)}{\partial \gamma_{1} \partial \beta} \approx \sum_{j=1}^{b} \frac{\left[\sum_{k 2=1}^{n_{k 2}} w_{2, k 2} P_{2}\right] \times D-E \times F}{\left[\sum_{k 2=1}^{n_{k 2}} w_{2, k 2} P_{2}\right]^{2}}
$$

where

$$
\begin{aligned}
& D=\sum_{k 2=1}^{n_{k 2}} w_{2, k 2} P_{2}\left[Q_{1,0,1,0}+Q_{0,0,0,1} Q_{1,0,1,0}+D_{23}\right] \\
& E=\sum_{k 2=1}^{n_{k 2}} w_{2, k 2} P_{2} Q_{1,0,1,0} \\
& F=\sum_{k 2=1}^{n_{k 2}} w_{2, k 2} P_{2} Q_{0,0,0,1}
\end{aligned}
$$


Jennifer L. K. Kensler Appendix B. Derivation of Variance-Covariance Matrix

$$
\frac{\partial^{2} \mathcal{L}\left(\boldsymbol{\gamma}, \beta, \sigma_{\rho}, \sigma_{\omega} \mid \text { Data }\right)}{\partial \gamma_{1} \partial \sigma_{\rho}} \approx \sqrt{2} \beta^{2} \sum_{j=1}^{b} \frac{\left[\sum_{k 2=1}^{n_{k 2}} w_{2, k 2} P_{2}\right] \times D-E \times F}{\left[\sum_{k 2=1}^{n_{k 2}} w_{2, k 2} P_{2}\right]^{2}}
$$

where

$$
\begin{aligned}
& D=\sum_{k 2=1}^{n_{k 2}} w_{2, k 2} q_{2, k 2} P_{2}\left[Q_{0,0,1,0} Q_{1,0,1,0}+D_{12}\right] \\
& E=\sum_{k 2=1}^{n_{k 2}} w_{2, k 2} P_{2} Q_{1,0,1,0} \\
& F=\sum_{k 2=1}^{n_{k 2}} w_{2, k 2} q_{2, k 2} P_{2} Q_{0,0,1,0}
\end{aligned}
$$

$$
\frac{\partial^{2} \mathcal{L}\left(\gamma, \beta, \sigma_{\rho}, \sigma_{\omega} \mid \text { Data }\right)}{\partial \gamma_{1} \partial \sigma_{\omega}} \approx \sqrt{2} \beta^{2} \sum_{j=1}^{b} \frac{\left[\sum_{k 2=1}^{n_{k 2}} w_{2, k 2} P_{2}\right] \times D-E \times F}{\left[\sum_{k 2=1}^{n_{k 2}} w_{2, k 2} P_{2}\right]^{2}}
$$

where

$$
\begin{aligned}
& D=\sum_{k 2=1}^{n_{k 2}} w_{2, k 2} P_{2}\left[Q_{0,1,1,0} Q_{1,0,1,0}+D_{25}\right] \\
& E=\sum_{k 2=1}^{n_{k 2}} w_{2, k 2} P_{2} Q_{1,0,1,0} \\
& F=\sum_{k 2=1}^{n_{k 2}} w_{2, k 2} P_{2} Q_{0,1,1,0}
\end{aligned}
$$


Jennifer L. K. Kensler Appendix B. Derivation of Variance-Covariance Matrix

$$
\frac{\partial^{2} \mathcal{L}\left(\boldsymbol{\gamma}, \beta, \sigma_{\rho}, \sigma_{\omega} \mid D a t a\right)}{\partial \beta^{2}} \approx \frac{1}{\beta^{2}} \sum_{j=1}^{b} \frac{\left[\sum_{k 2=1}^{n_{k 2}} w_{2, k 2} P_{2}\right] \times D-E \times F}{\left[\sum_{k 2=1}^{n_{k 2}} w_{2, k 2} P_{2}\right]^{2}}
$$

where

$$
\begin{aligned}
& D=\sum_{k 2=1}^{n_{k 2}} w_{2, k 2} P_{2}\left[-Q_{0,0,0,1}+Q_{0,0,0,1}^{2}+D_{33}\right] \\
& E=\sum_{k 2=1}^{n_{k 2}} w_{2, k 2} P_{2} Q_{0,0,0,1} \\
& F=\sum_{k 2=1}^{n_{k 2}} w_{2, k 2} P_{2} Q_{0,0,0,1}
\end{aligned}
$$

$$
\frac{\partial^{2} \mathcal{L}\left(\boldsymbol{\gamma}, \beta, \sigma_{\rho}, \sigma_{\omega} \mid \text { Data }\right)}{\partial \beta \partial \sigma_{\rho}} \approx \sqrt{2} \sum_{j=1}^{b} \frac{\left[\sum_{k 2=1}^{n_{k 2}} w_{2, k 2} P_{2}\right] \times D-E \times F}{\left[\sum_{k 2=1}^{n_{k 2}} w_{2, k 2} P_{2}\right]^{2}}
$$

where

$$
\begin{aligned}
& D=\sum_{k 2=1}^{n_{k 2}} w_{2, k 2} q_{2, k 2} P_{2}\left[Q_{0,0,1,0} Q_{0,0,0,1}+D_{34}\right] \\
& E=\sum_{k 2=1}^{n_{k 2}} w_{2, k 2} P_{2} Q_{0,0,0,1} \\
& F=\sum_{k 2=1}^{n_{k 2}} w_{2, k 2} q_{2, k 2} P_{2} Q_{0,0,1,0}
\end{aligned}
$$


Jennifer L. K. Kensler Appendix B. Derivation of Variance-Covariance Matrix

$$
\frac{\partial^{2} \mathcal{L}\left(\boldsymbol{\gamma}, \beta, \sigma_{\rho}, \sigma_{\omega} \mid \text { Data }\right)}{\partial \beta \partial \sigma_{\omega}} \approx \sqrt{2} \sum_{j=1}^{b} \frac{\left[\sum_{k 2=1}^{n_{k 2}} w_{2, k 2} P_{2}\right] \times D-E \times F}{\left[\sum_{k 2=1}^{n_{k 2}} w_{2, k 2} P_{2}\right]^{2}}
$$

where

$$
\begin{aligned}
& D=\sum_{k 2=1}^{n_{k 2}} w_{2, k 2} P_{2}\left[Q_{0,1,1,0} Q_{0,0,0,1}+D_{35}\right] \\
& E=\sum_{k 2=1}^{n_{k 2}} w_{2, k 2} P_{2} Q_{0,0,0,1} \\
& F=\sum_{k 2=1}^{n_{k 2}} w_{2, k 2} P_{2} Q_{0,1,1,0}
\end{aligned}
$$

$$
\frac{\partial^{2} \mathcal{L}\left(\boldsymbol{\gamma}, \beta, \sigma_{\rho}, \sigma_{\omega} \mid \text { Data }\right)}{\partial \sigma_{\rho}^{2}} \approx 2 \beta^{2} \sum_{j=1}^{b} \frac{\left[\sum_{k 2=1}^{n_{k 2}} w_{2, k 2} P_{2}\right] \times D-E \times F}{\left[\sum_{k 2=1}^{n_{k 2}} w_{2, k 2} P_{2}\right]^{2}}
$$

where

$$
\begin{aligned}
& D=\sum_{k 2=1}^{n_{k 2}} w_{2, k 2} q_{2, k 2}^{2} P_{2}\left[Q_{0,0,1,0}^{2}+D_{11}\right] \\
& E=\sum_{k 2=1}^{n_{k 2}} w_{2, k 2} q_{2, k 2} P_{2} Q_{0,0,1,0} \\
& F=\sum_{k 2=1}^{n_{k 2}} w_{2, k 2} q_{2, k 2} P_{2} Q_{0,0,1,0}
\end{aligned}
$$


Jennifer L. K. Kensler Appendix B. Derivation of Variance-Covariance Matrix

$$
\frac{\partial^{2} \mathcal{L}\left(\boldsymbol{\gamma}, \beta, \sigma_{\rho}, \sigma_{\omega} \mid \text { Data }\right)}{\partial \sigma_{\rho} \partial \sigma_{\omega}} \approx 2 \beta^{2} \sum_{j=1}^{b} \frac{\left[\sum_{k 2=1}^{n_{k 2}} w_{2, k 2} P_{2}\right] \times D-E \times F}{\left[\sum_{k 2=1}^{n_{k 2}} w_{2, k 2} P_{2}\right]^{2}}
$$

where

$$
\begin{aligned}
& D=\sum_{k 2=1}^{n_{k 2}} w_{2, k 2} q_{2, k 2}^{2} P_{2}\left[Q_{0,1,1,0} Q_{0,0,1,0}+D_{15}\right] \\
& E=\sum_{k 2=1}^{n_{k 2}} w_{2, k 2} q_{2, k 2} P_{2} Q_{0,0,1,0} \\
& F=\sum_{k 2=1}^{n_{k 2}} w_{2, k 2} q_{2, k 2} P_{2} Q_{0,1,1,0}
\end{aligned}
$$

$$
\frac{\partial^{2} \mathcal{L}\left(\boldsymbol{\gamma}, \beta, \sigma_{\rho}, \sigma_{\omega} \mid \text { Data }\right)}{\partial \sigma_{\omega}^{2}} \approx 2 \beta^{2} \sum_{j=1}^{b} \frac{\left[\sum_{k 2=1}^{n_{k 2}} w_{2, k 2} P_{2}\right] \times D-E \times F}{\left[\sum_{k 2=1}^{n_{k 2}} w_{2, k 2} P_{2}\right]^{2}}
$$

where

$$
\begin{aligned}
& D=\sum_{k 2=1}^{n_{k 2}} w_{2, k 2} P_{2}\left[Q_{0,1,1,0}^{2}+D_{55}\right] \\
& E=\sum_{k 2=1}^{n_{k 2}} w_{2, k 2} P_{2} Q_{0,1,1,0} \\
& F=\sum_{k 2=1}^{n_{k 2}} w_{2, k 2} P_{2} Q_{0,1,1,0}
\end{aligned}
$$




\section{Appendix C}

\section{$\mathrm{R}$ and SAS Code}

\section{C.1 R Code}

library (stats4)

library (glmmL)

\#\#\#Perform Gauss-Hermite Quadrature on Battery Life data for various number of quadrature points. \#\#\#Import Battery Life Data

data<-read.table("C:/Users/.../Battery Life.txt",header=TRUE)

\#\#\#Set number of test stand effect quadrature points

nq1<-20

\#\#\#Quadrature Points for test stand effect

qk1<-ghq (nq1, modified=FALSE) \$zeros

$\mathrm{qk} 1<-\mathrm{t}$ (qk1)

\#\#\#Weights of Quadrature Points for test stand effect

wk1<-ghq(nq1, modified=FALSE) \$weights

wk1<-t $($ wk1)

\#\#\#Set number of block effect quadrature points

nq2 $<-20$

\#\#Quadrature Points for block effect

qk2<-ghq (nq2, modified=FALSE) \$zeros

$\mathrm{qk} 2<-\mathrm{t}(\mathrm{qk} 2)$ 


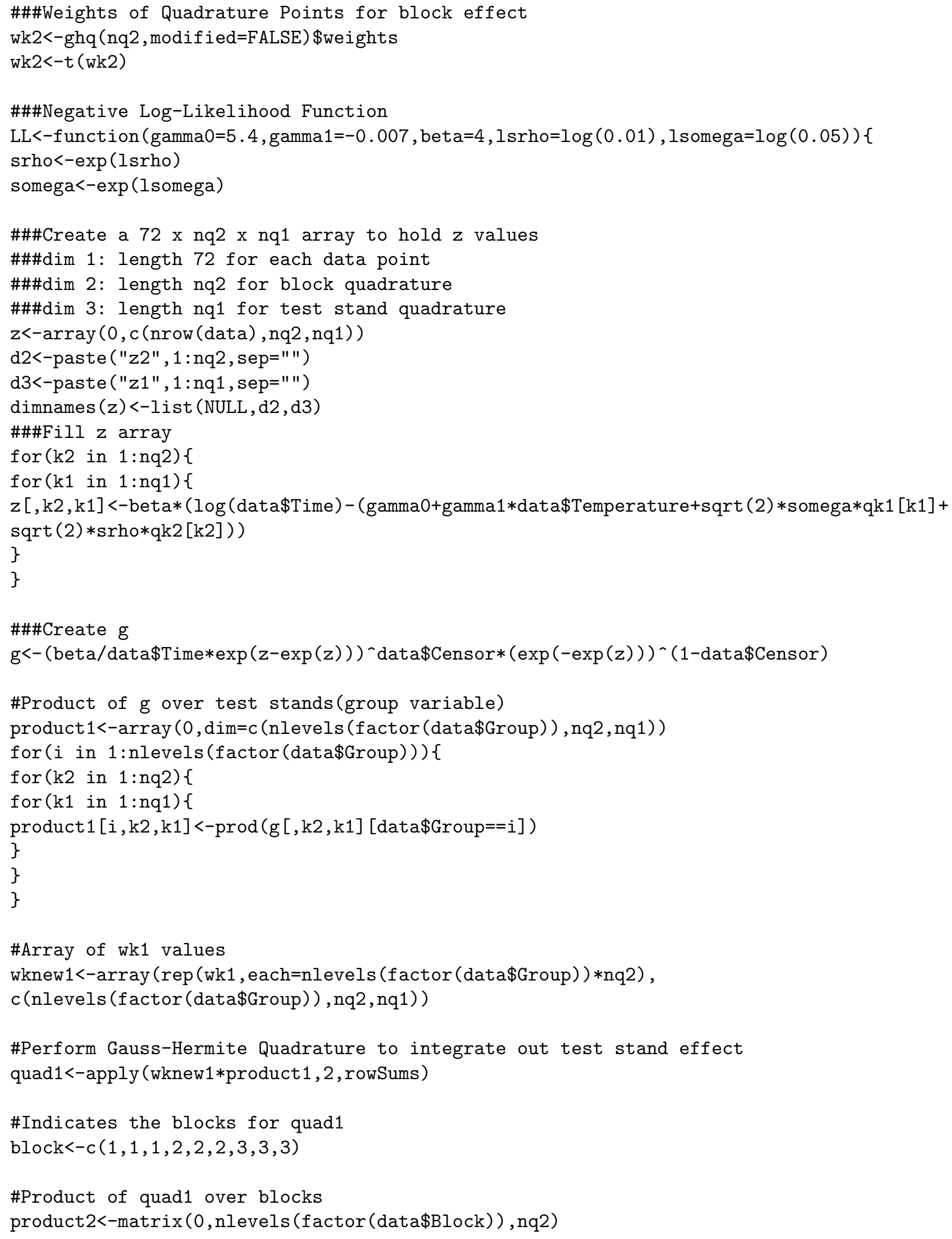




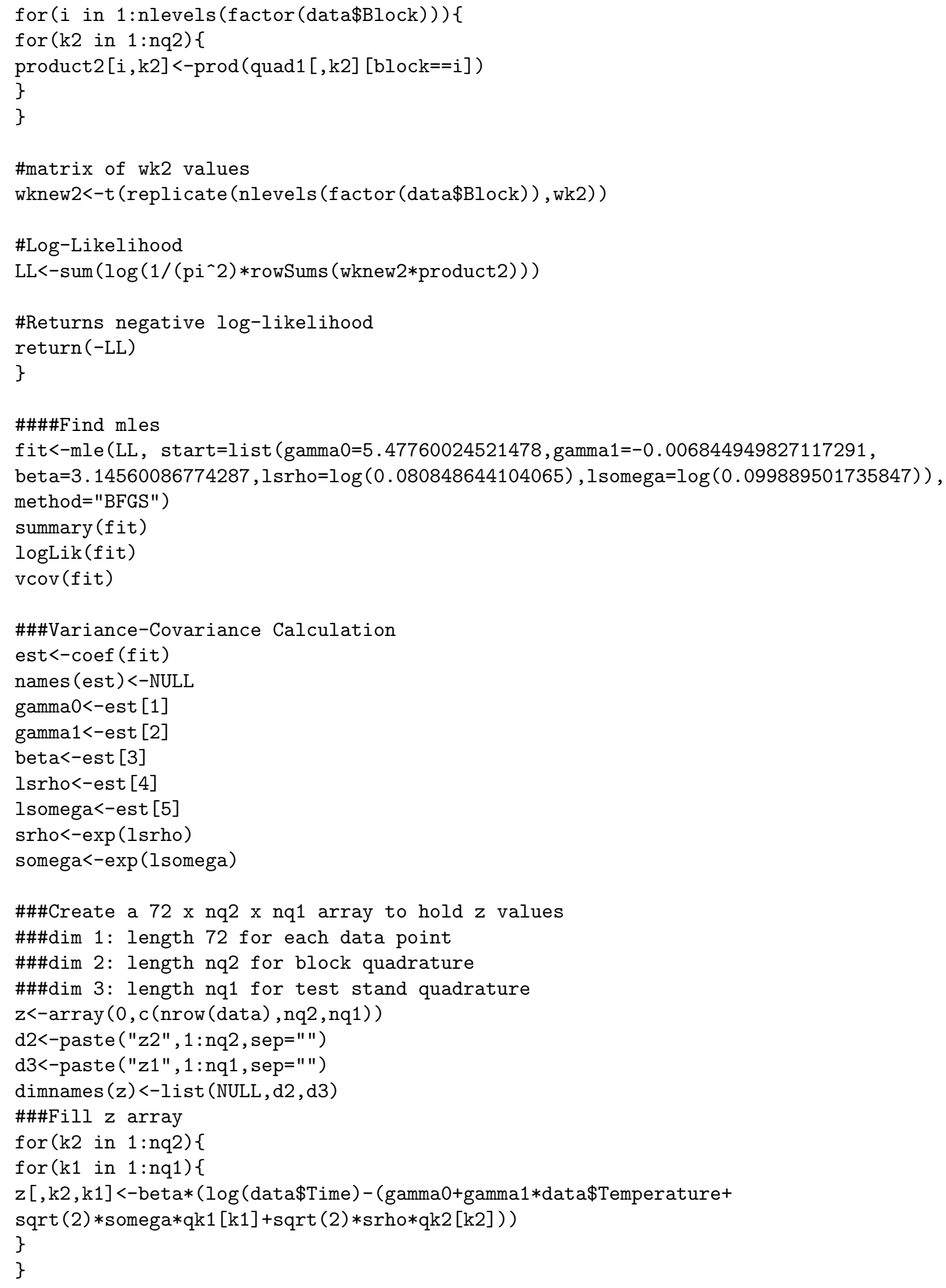




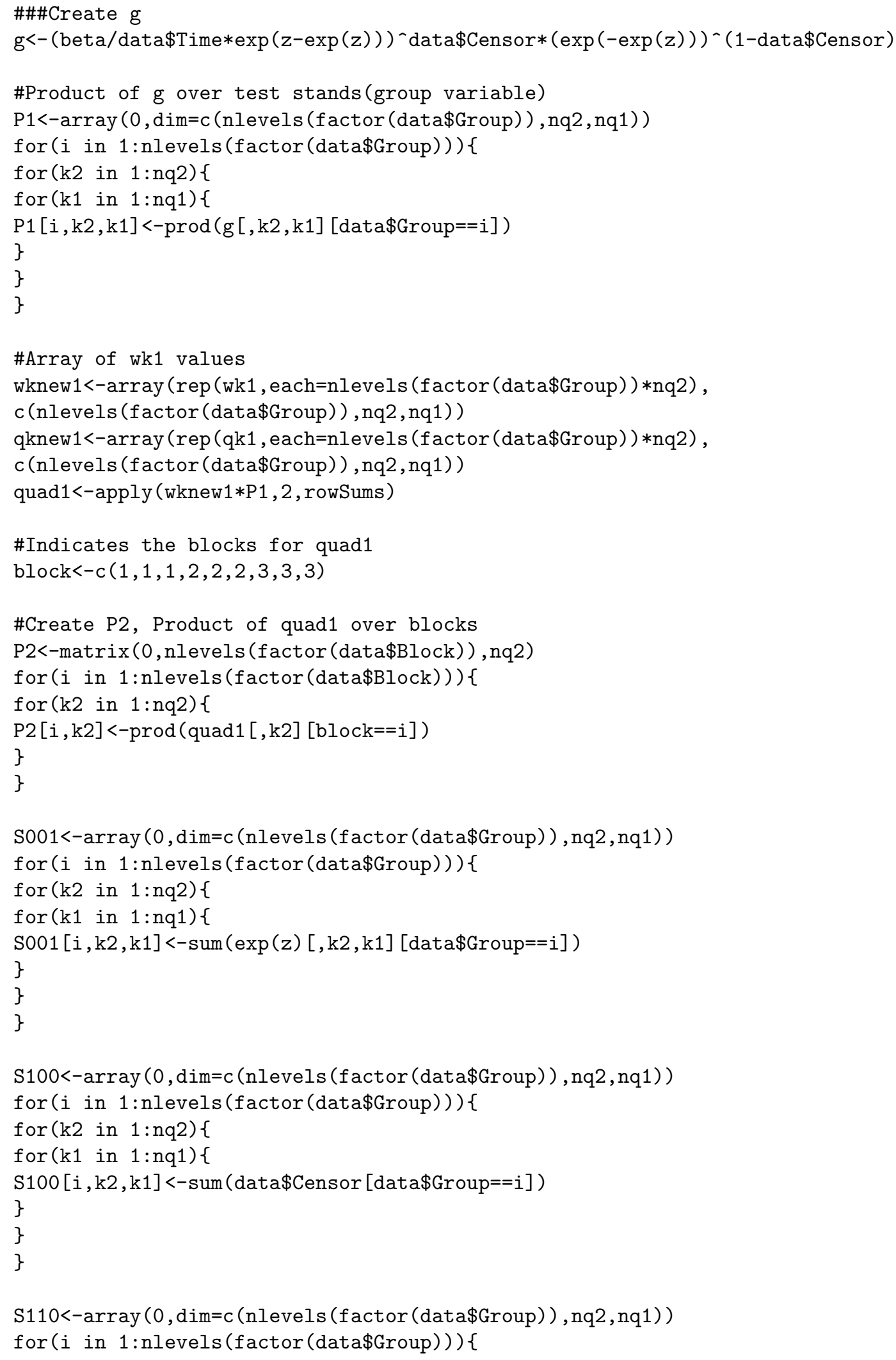




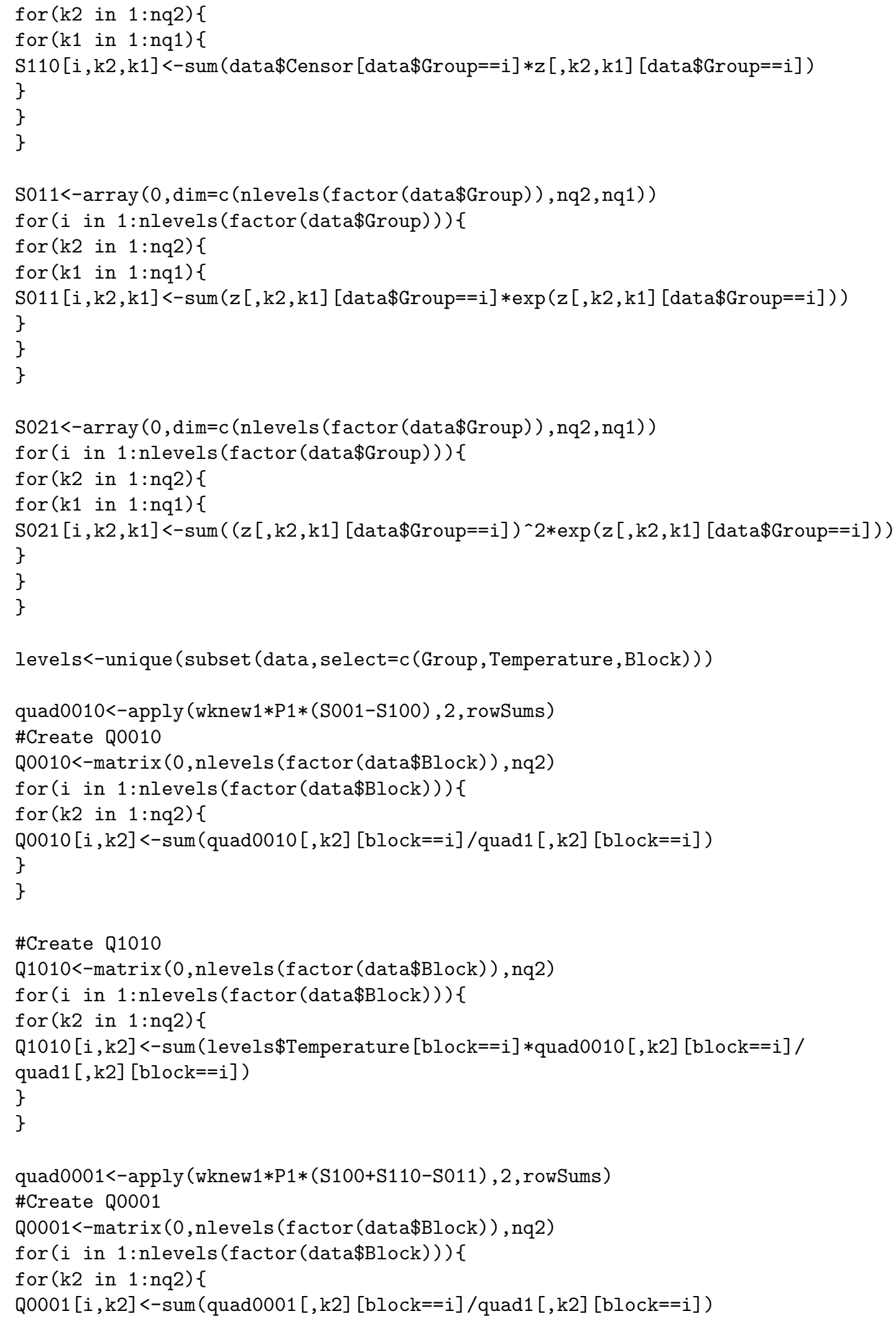


\}

\}

quad0110<-apply (wknew $1 *$ qknew $1 * \mathrm{P} 1 *(\mathrm{~S} 001-\mathrm{S} 100), 2$, rowSums)

\#Create Q0110

Q0110<-matrix (0,nlevels (factor (data\$Block)), nq2)

for ( $i$ in $1:$ nlevels (factor (data $\$ B l o c k))$ ) \{

for (k2 in $1: n q 2)\{$

Q0110 [i,k2]<-sum (quad0110 [,k2] [block==i] /quad1 [,k2] [block==i])

\}

\}

d11<-apply (wknew1*P1*((S001-S100)^2-S001), 2, rowSums)

D11<-matrix $(0$, nlevels (factor (data\$Block)), nq2)

for (i in 1:nlevels (factor (data $\$ B l o c k)$ )) \{

for $(\mathrm{k} 2$ in $1: \mathrm{nq} 2)\{$

$\mathrm{D} 11[\mathrm{i}, \mathrm{k} 2]<-\operatorname{sum}(($ quad1 $[, \mathrm{k} 2][\mathrm{block}==\mathrm{i}] * \mathrm{~d} 11[, \mathrm{k} 2][\mathrm{block}==\mathrm{i}]-($ quad0010 [, k2] [block==i]) 2$) /$

(quad1 $\left.[, \mathrm{k} 2][\mathrm{block}==\mathrm{i}])^{\wedge} 2\right)$

\}

\}

D12<-matrix (0,nlevels (factor (data\$Block)), nq2)

for $(i$ in $1: n l e v e l s($ factor (data $\$ B l o c k)))\{$

for (k2 in $1: \mathrm{nq} 2)$ \{

$\mathrm{D} 12[\mathrm{i}, \mathrm{k} 2]<-\operatorname{sum}($ levels $\$$ Temperature [block==i] $*($ quad1 [, k2] [block==i] *d11[, k2] [block==i] $\left.\left.(\text { quad0010 }[, \mathrm{k} 2][\mathrm{block}==\mathrm{i}])^{-2} 2\right) /(\operatorname{quad} 1[, \mathrm{k} 2][\mathrm{block}==\mathrm{i}])^{\wedge} 2\right)$

\}

\}

d13<-apply (wknew1*P1*((S100+S110-S011)*(S001-S100)+S011), 2 , rowSums)

D13<-matrix $(0$, nlevels (factor (data\$Block)), nq2)

for (i in $1:$ nlevels (factor (data\$Block)) ) \{

for $(\mathrm{k} 2$ in $1: \mathrm{nq} 2)\{$

$\mathrm{D} 13[\mathrm{i}, \mathrm{k} 2]<-\operatorname{sum}(($ quad1 $[, \mathrm{k} 2][\mathrm{block}==\mathrm{i}] * \mathrm{~d} 13[, \mathrm{k} 2][\mathrm{block}==\mathrm{i}]$-quad0010 [, k2] [block==i]* quad0001 $\left.[, \mathrm{k} 2][\mathrm{block}==\mathrm{i}]) /(\text { quad1 }[, \mathrm{k} 2][\mathrm{block}==\mathrm{i}])^{\wedge} 2\right)$ \}

\}

d15<-apply (wknew1*qknew1*P1*((S001-S100)^2-S001), 2 , rowSums)

D15<-matrix (0,nlevels (factor (data\$Block)), nq2)

for (i in $1:$ nlevels (factor (data $\$ B l o c k)))\{$

for $(\mathrm{k} 2$ in $1: \mathrm{nq} 2)\{$

$\mathrm{D} 15[\mathrm{i}, \mathrm{k} 2]<-\operatorname{sum}($ (quad1 [, k2] [block==i] *d15[,k2] [block==i] -quad0010 [, k2] [block==i]* quad0110[,k2] [block==i])/(quad1 [,k2] [block==i])^2)

\}

\}

D22<-matrix (0,nlevels (factor (data\$Block)), nq2)

for (i in 1:nlevels (factor (data\$Block)) ) \{

for $(\mathrm{k} 2$ in $1: \mathrm{nq} 2)$ \{

D22 [i,k2]<-sum ((levels\$Temperature [block==i] ) $2 *($ quad1 [, k2] [block==i] * 


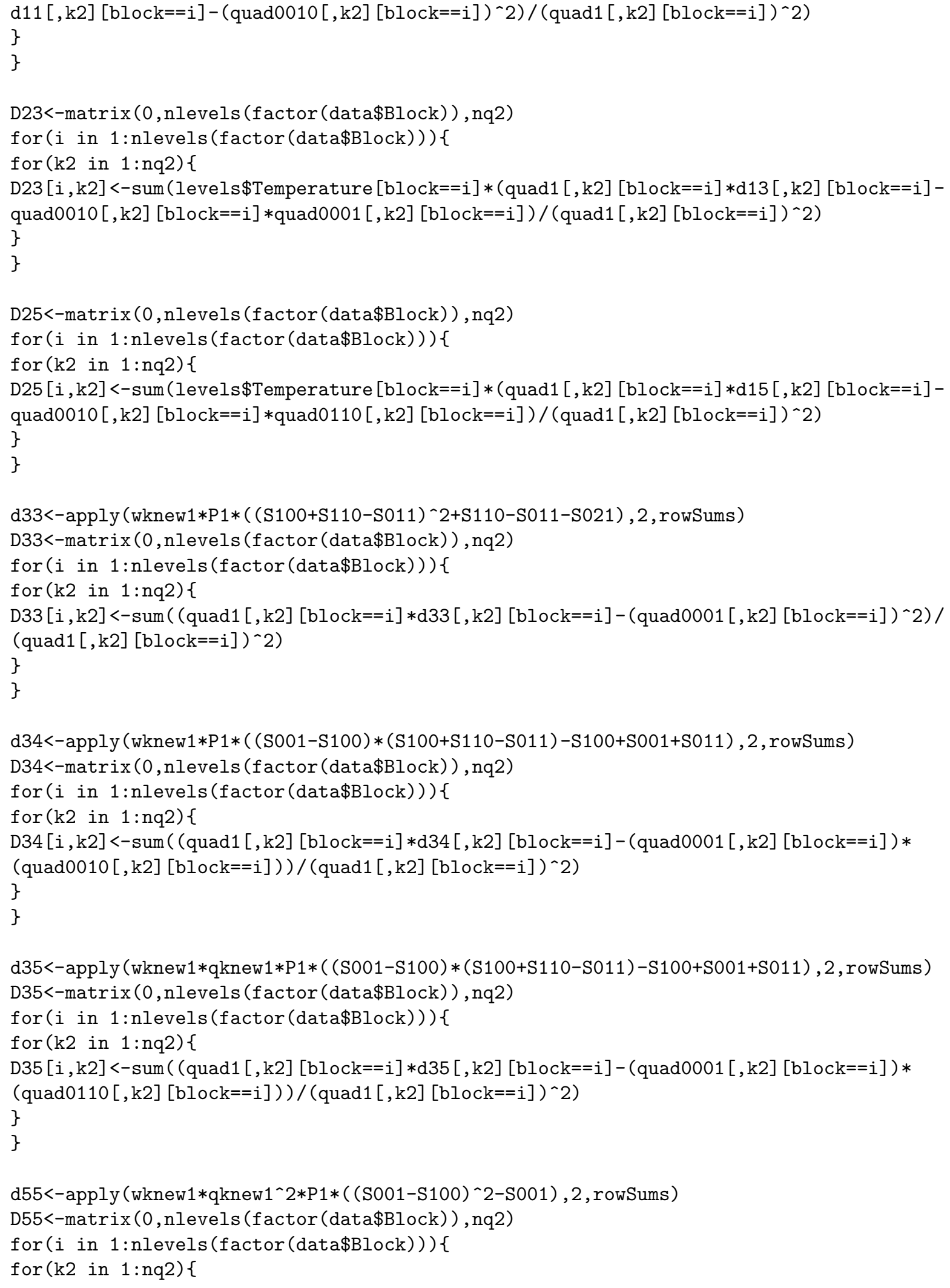




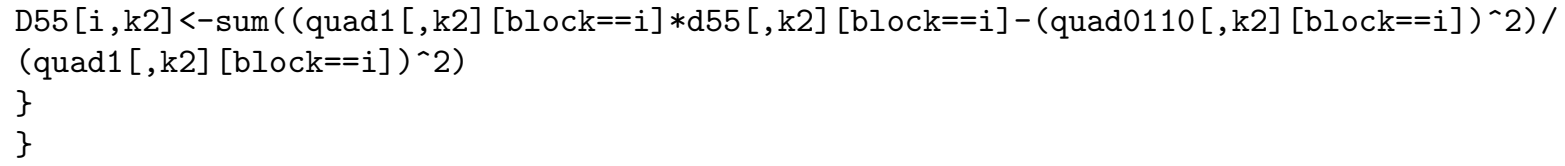




\section{C.2 SAS Code}

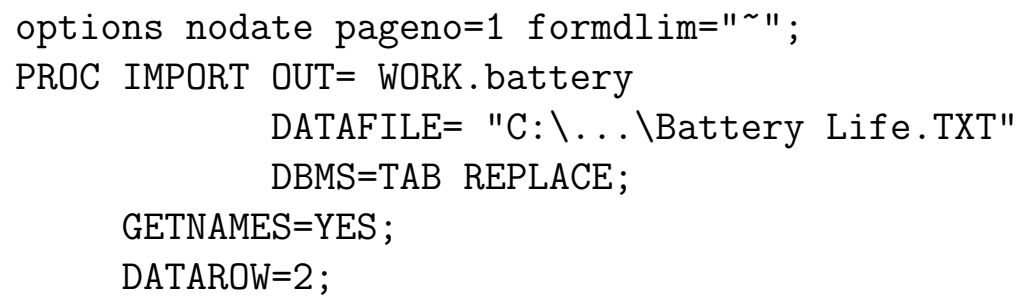




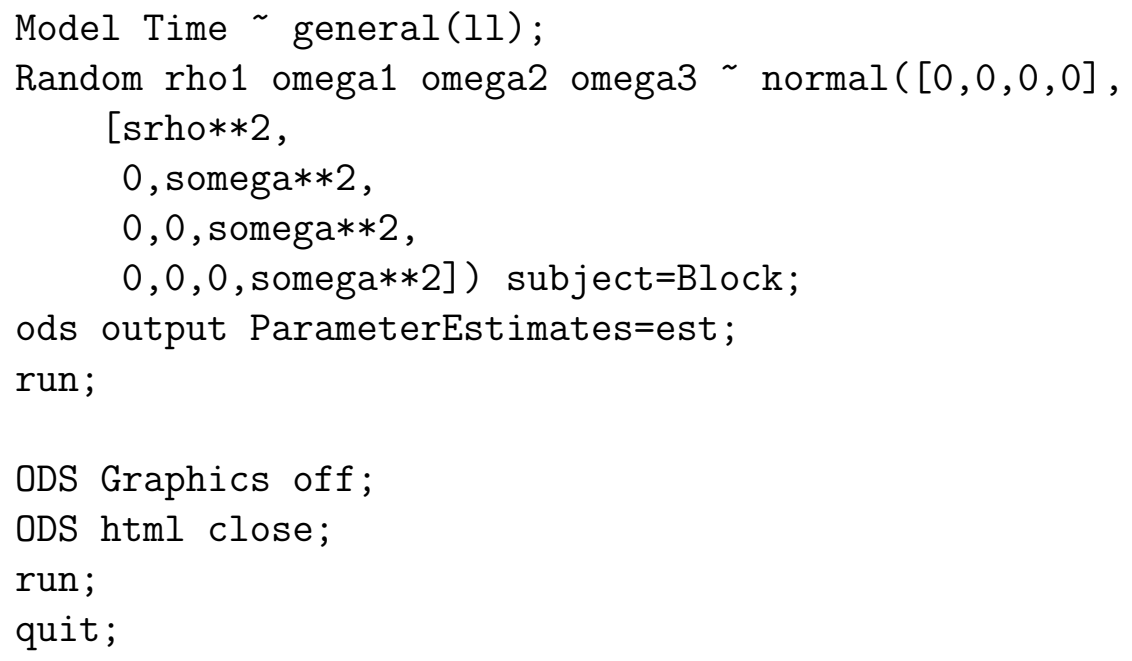




\section{Appendix D}

\section{Weibull Variability}

The following tables examine the distribution of the standard deviation of failure times on a test stand. Data were simulated in the same manner as Section 6.1. The standard deviation of failure times was then calculated for each test stand. For both the censored and uncensored

case $n=8$, and for the censored case $r=4$. The following figures show boxplots for the standard deviation of test stand failure times. 
Figure D.1: Test Stand Standard Deviation by $\beta$

(a) Uncensored

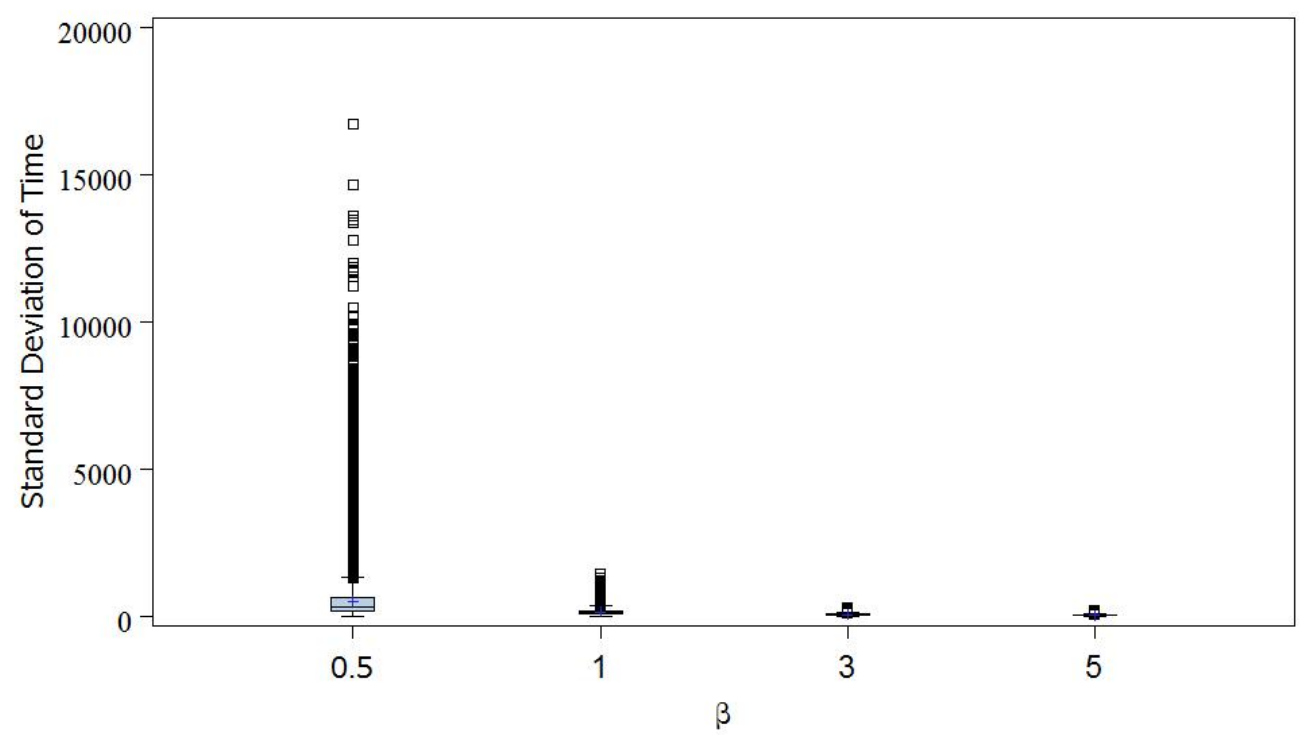

(b) Censored

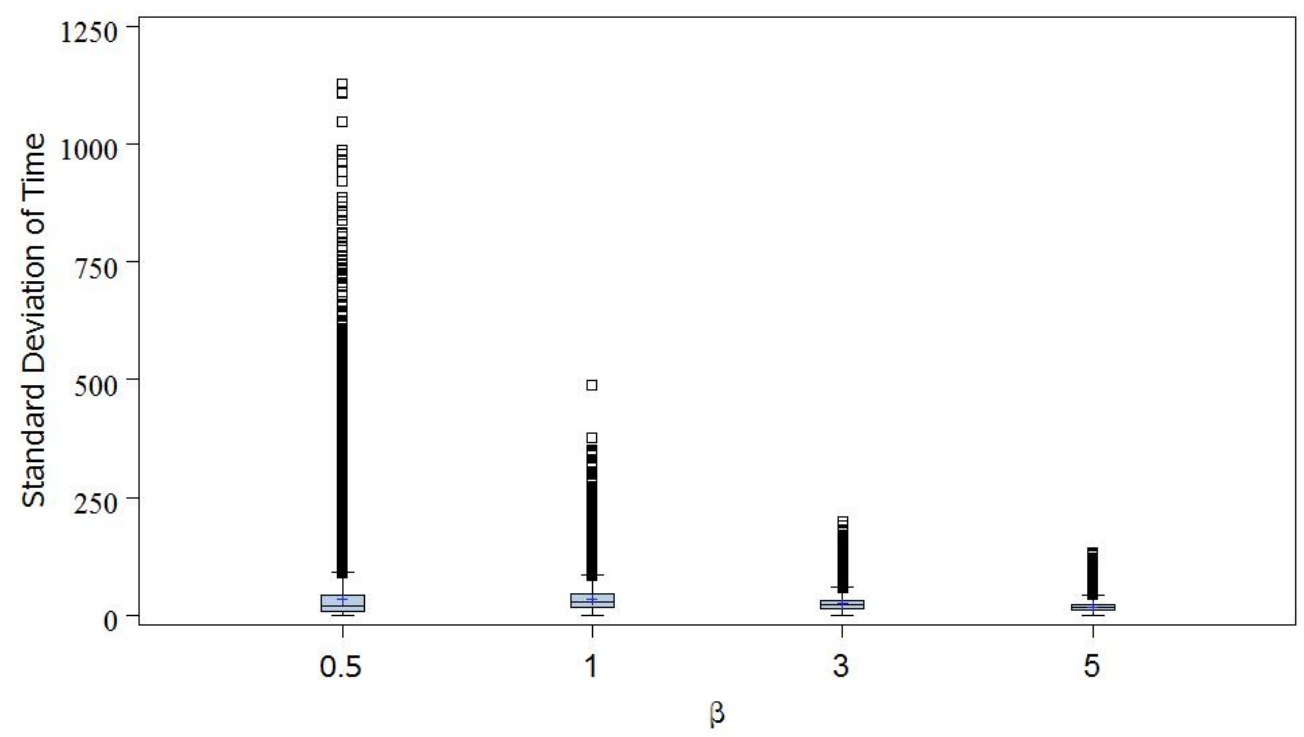


Figure D.2: Test Stand Standard Deviation by $x$

(a) Uncensored

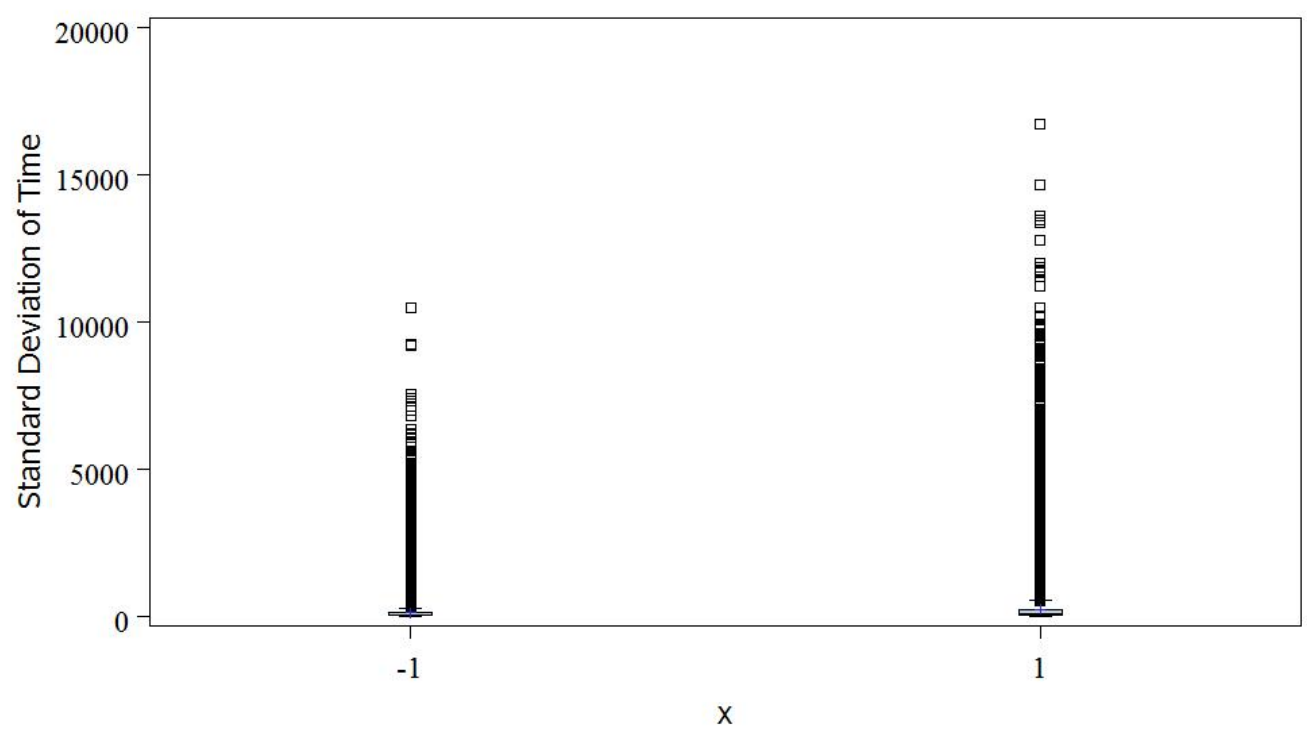

(b) Censored

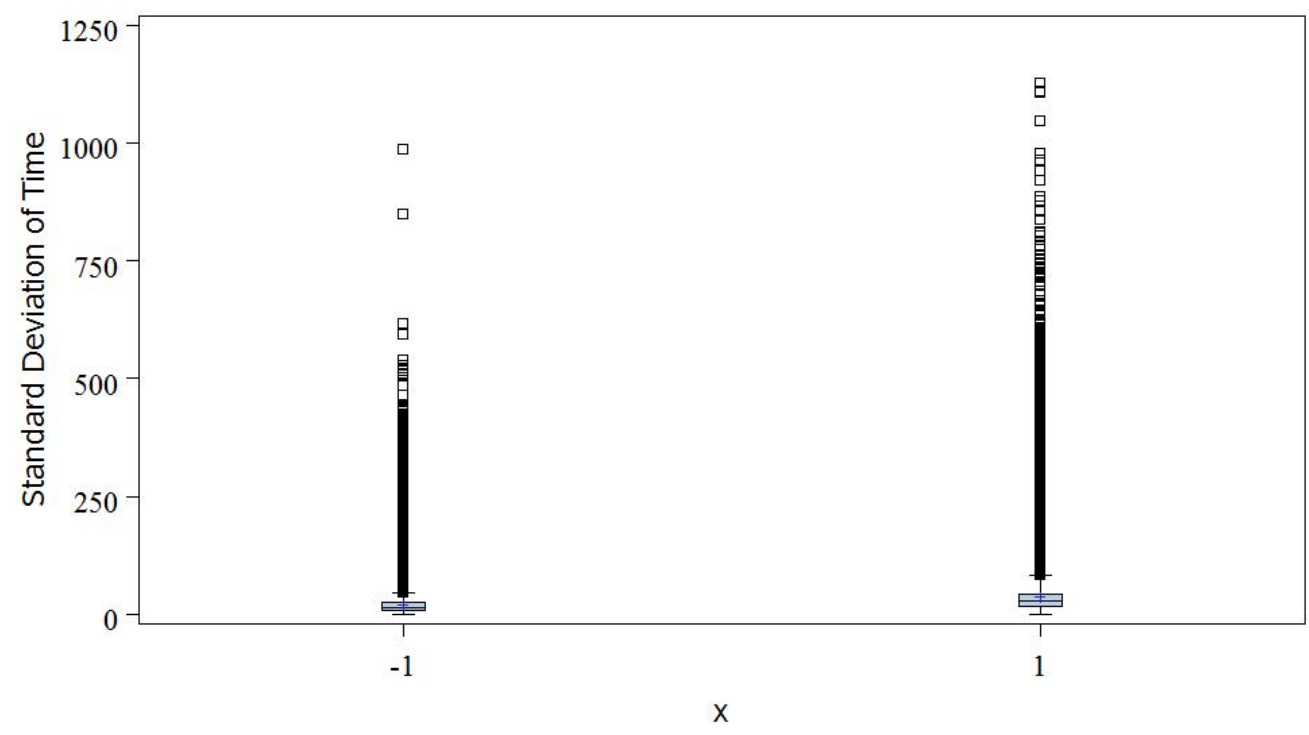


Figure D.3: Test Stand Standard Deviation by $x$ and $\beta$

(a) Uncensored
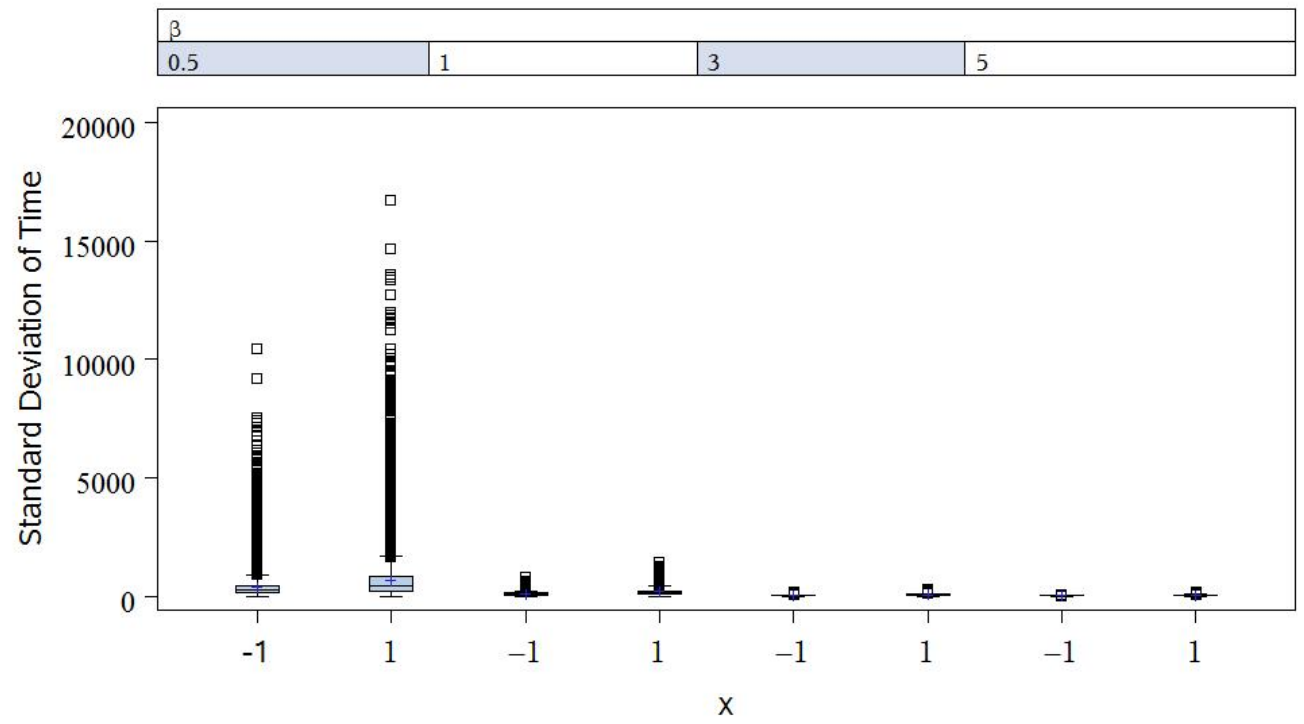

(b) Censored
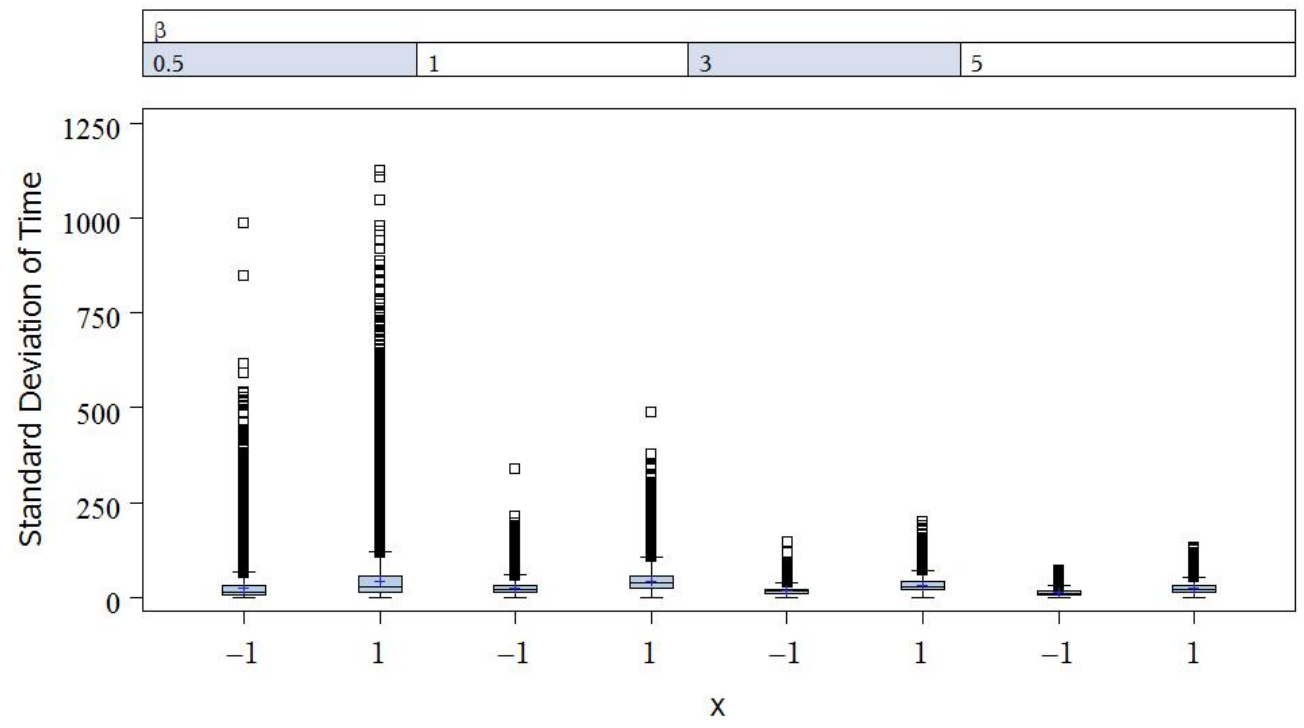
Figure D.4: Test Stand Standard Deviation by $\sigma_{\rho}$

(a) Uncensored

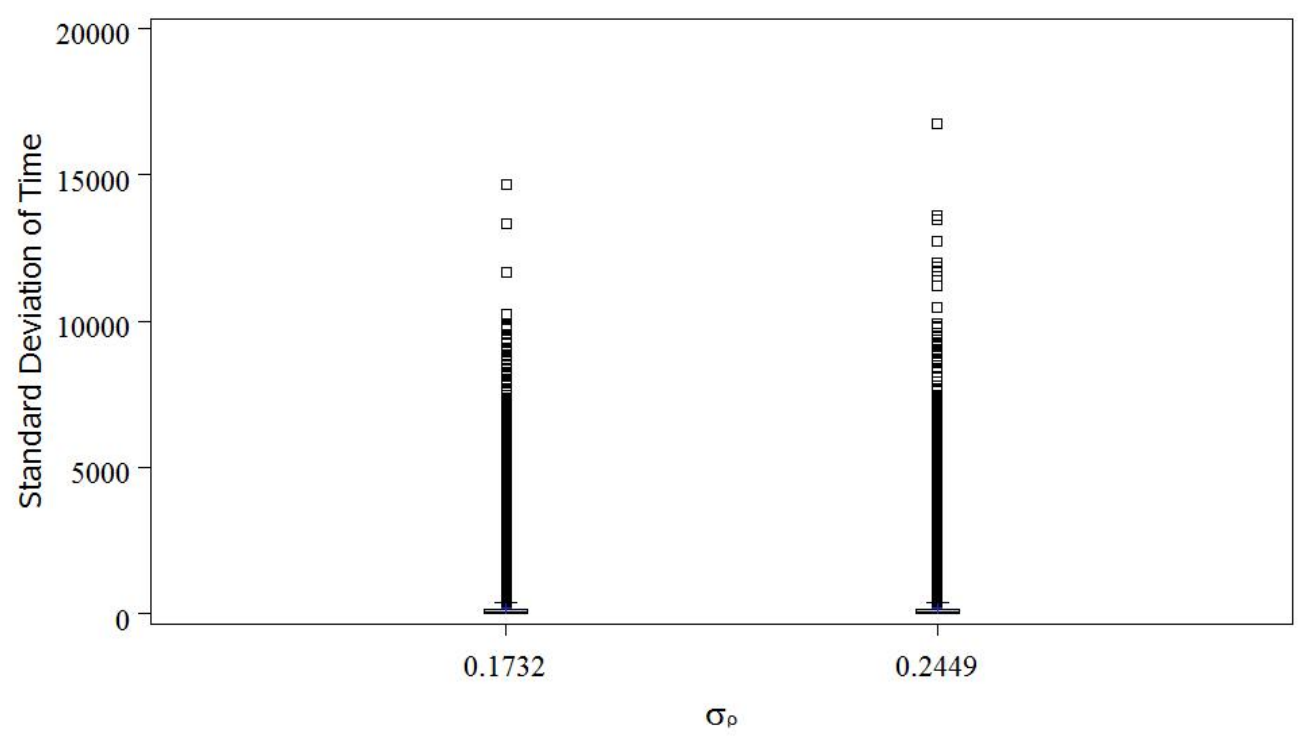

(b) Censored

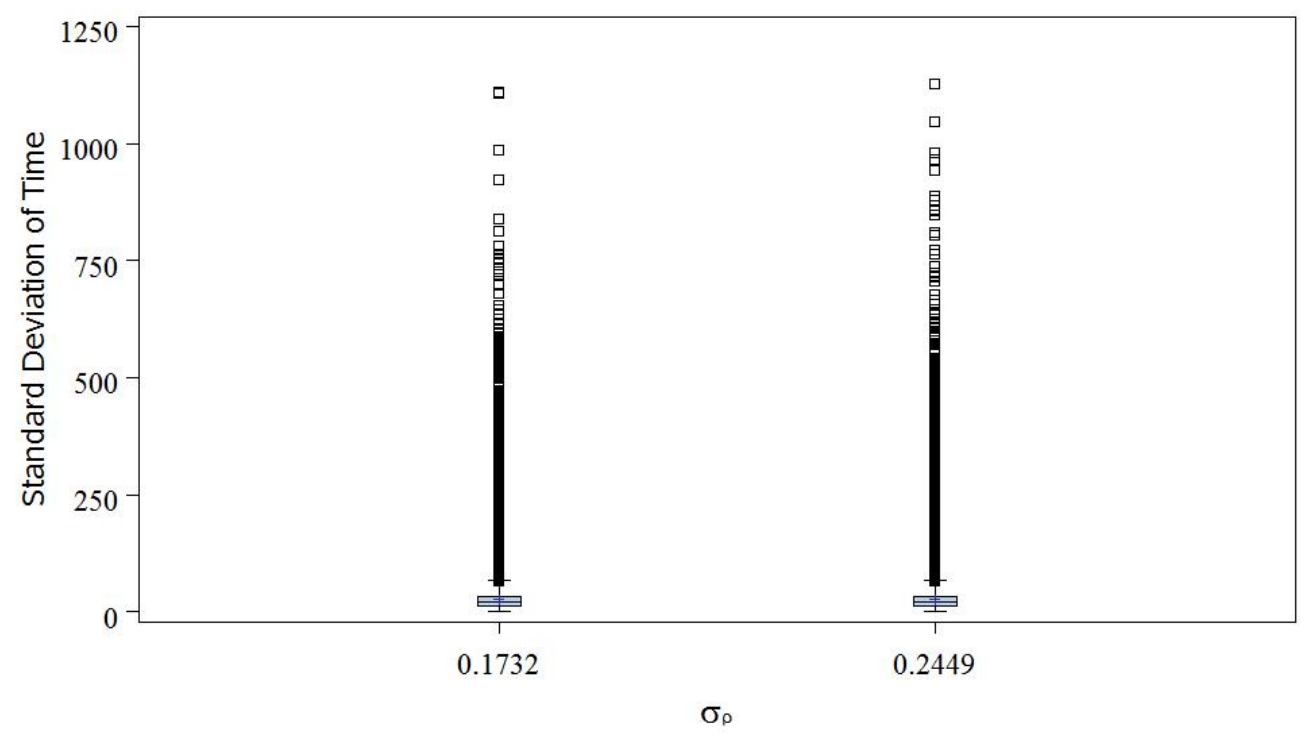


Figure D.5: Test Stand Standard Deviation by $\sigma_{\rho}$ and $\beta$

(a) Uncensored
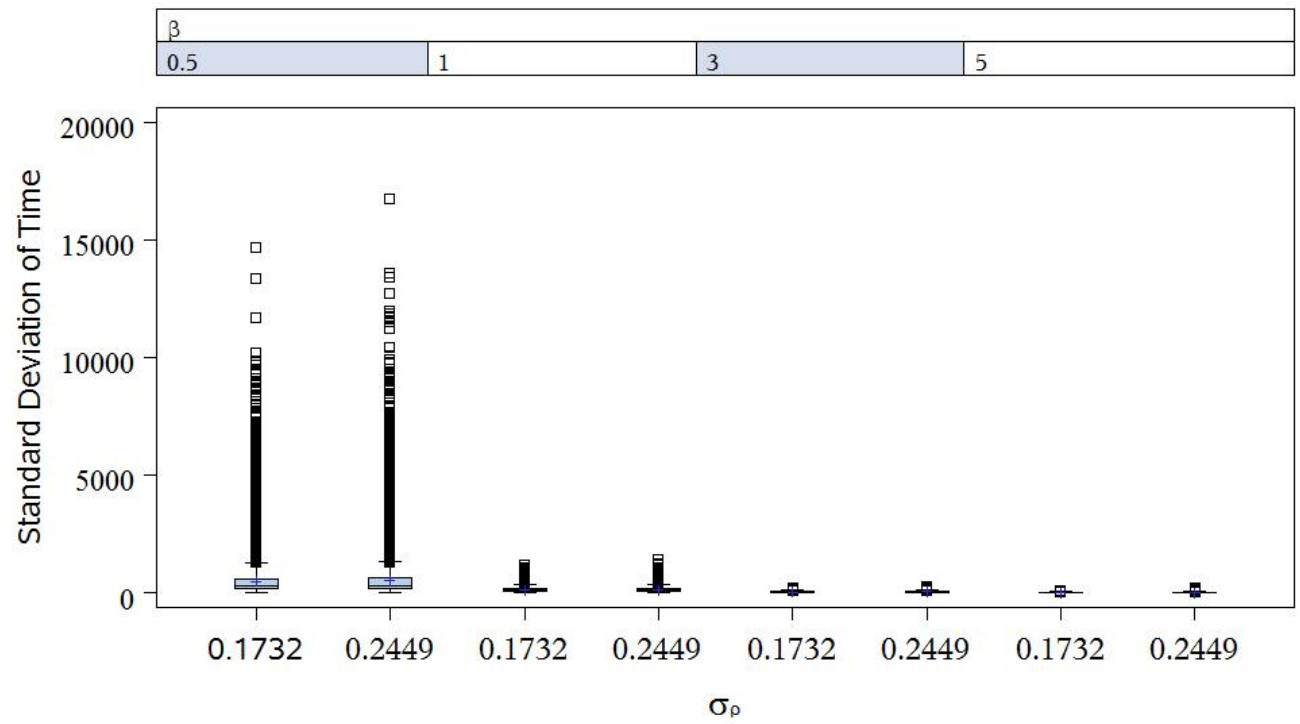

(b) Censored
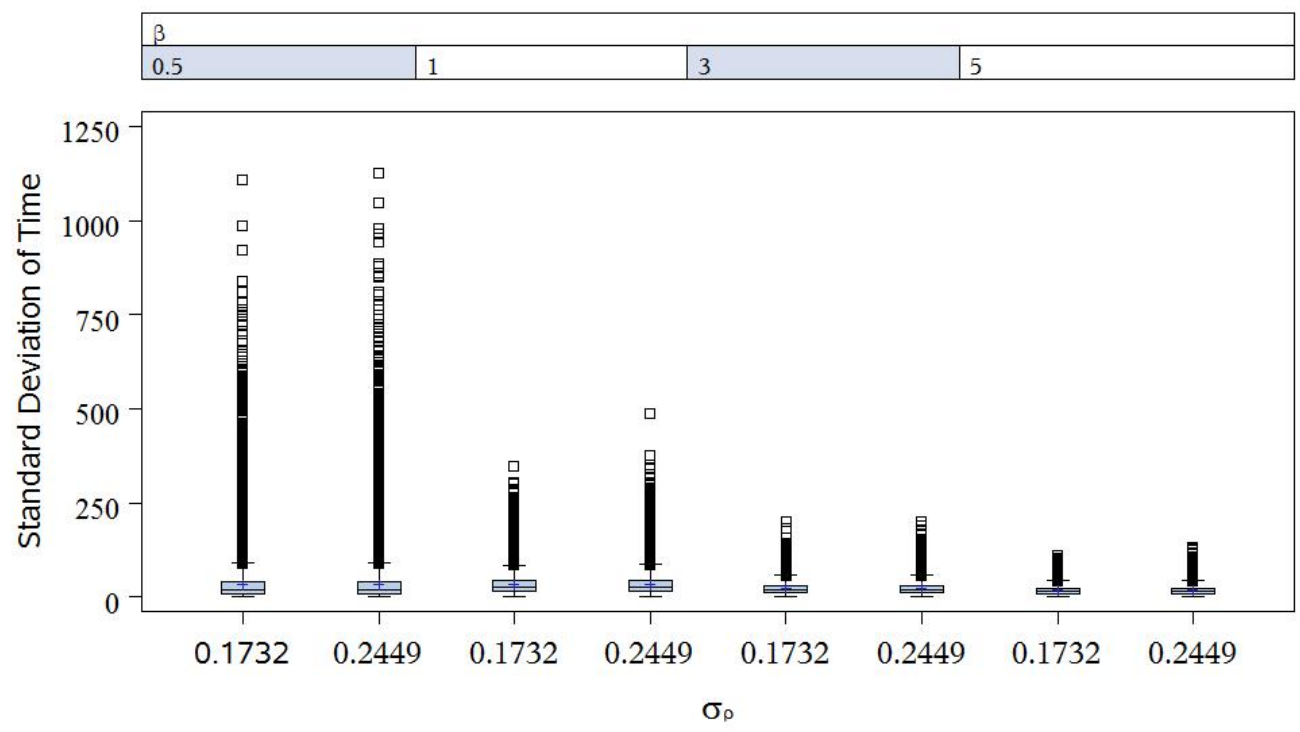
Figure D.6: Test Stand Standard Deviation by $\sigma_{\omega}$

(a) Uncensored

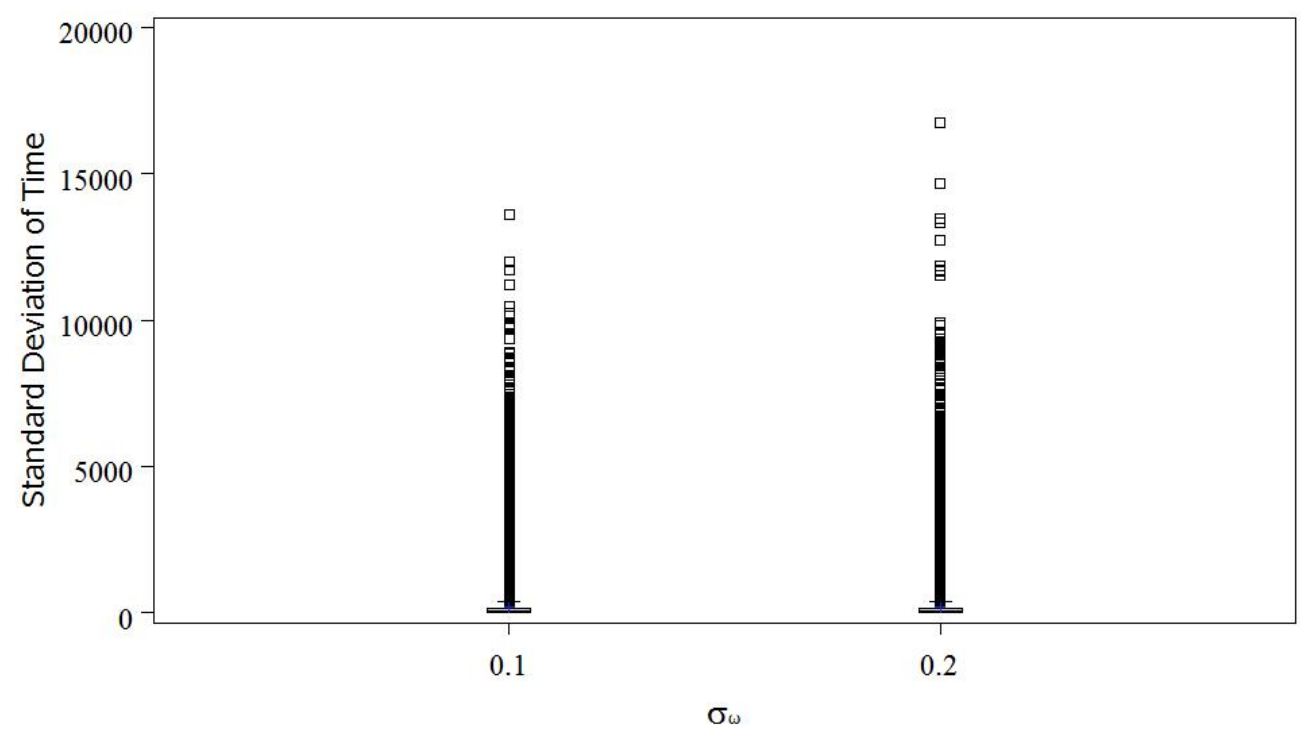

(b) Censored

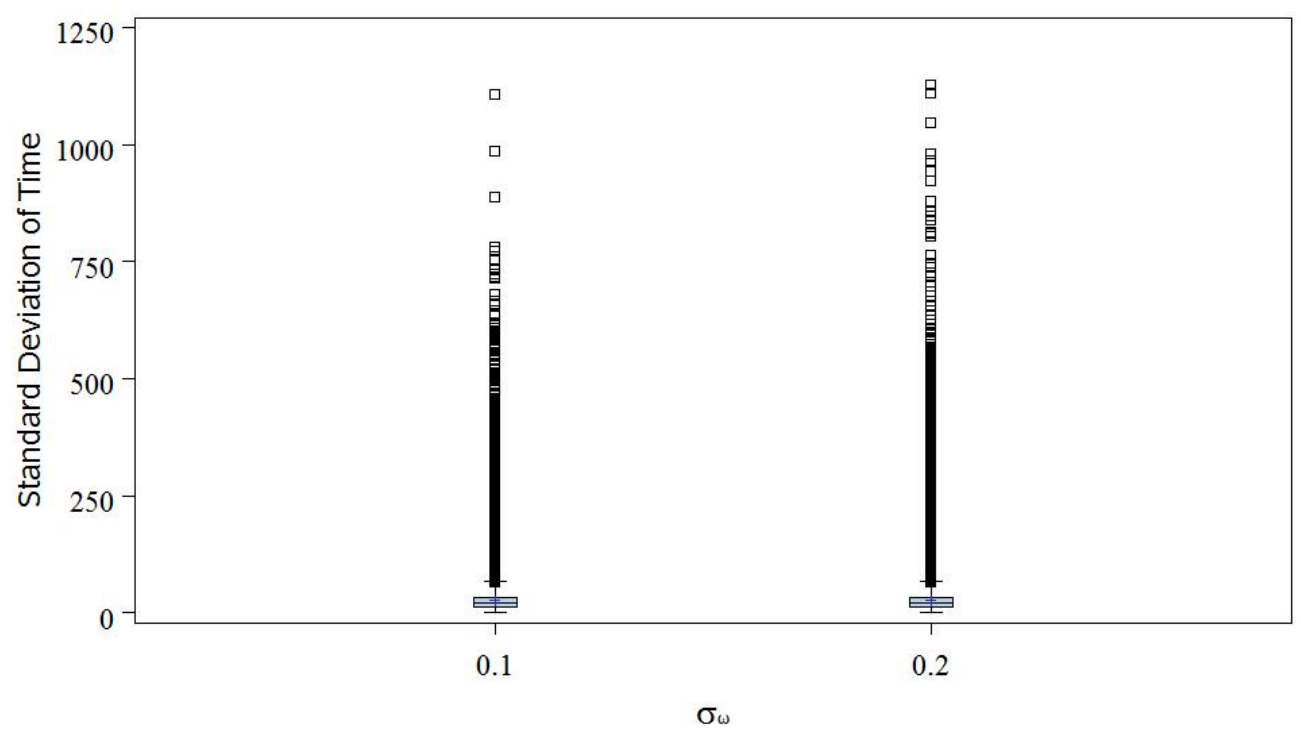


Figure D.7: Test Stand Standard Deviation by $\sigma_{\omega}$ and $\beta$

(a) Uncensored
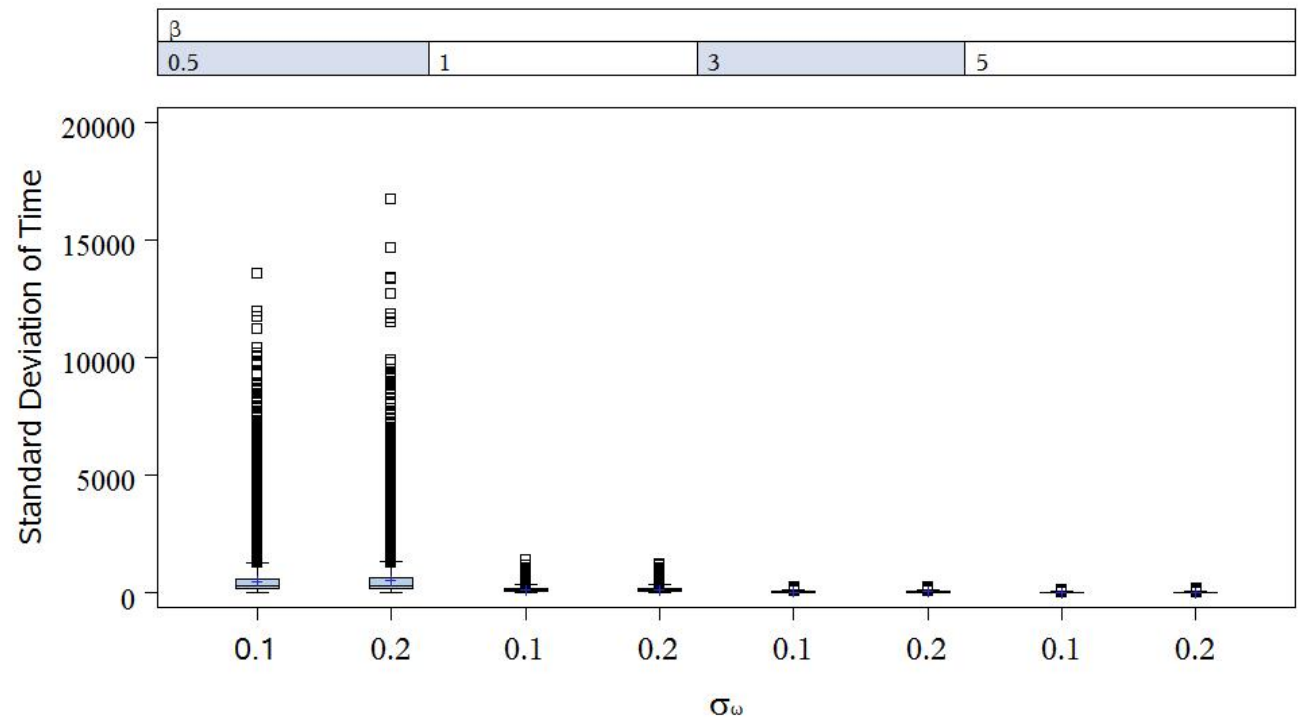

(b) Censored
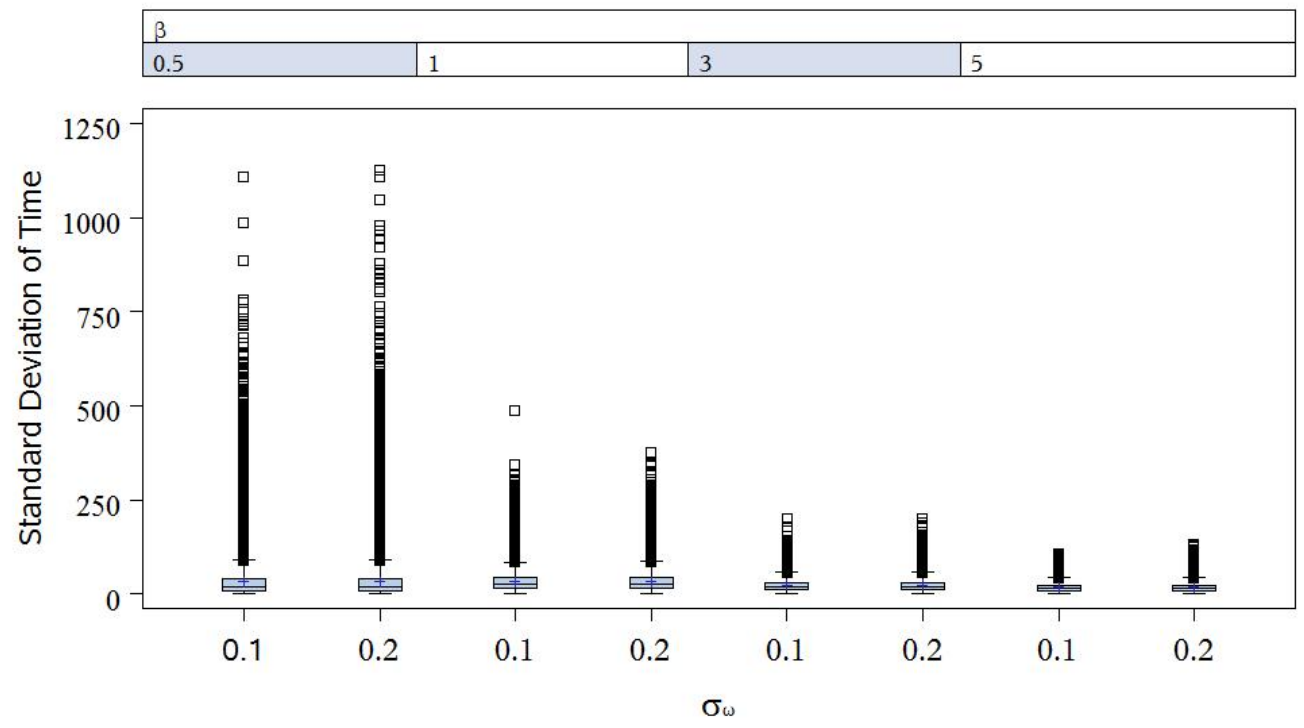